\title{
Utilização de web semântica para seleção de informações de web services no registro UDDI uma abordagem com qualidade de serviço
}

\author{
Luis Hideo Vasconcelos Nakamura
}



Data de Depósito:

Assinatura:

\title{
Utilização de web semântica para seleção de informações de web services no registro UDDI uma abordagem com qualidade de serviço
}

\author{
Luis Hideo Vasconcelos Nakamura
}

Orientador: Prof. Dr. Marcos José Santana

Dissertação apresentada ao Instituto de Ciências Matemáticas e de Computação - ICMC-USP, como parte dos requisitos para obtenção do título de Mestre em Ciências - Ciências de Computação e Matemática Computacional. VERSÃO REVISADA

USP - São Carlos

Fevereiro de 2012 
Ficha catalográfica elaborada pela Biblioteca Prof. Achille Bassi e Seção Técnica de Informática, ICMC/USP, com os dados fornecidos pelo(a) autor(a)

\begin{tabular}{|c|c|}
\hline \multirow[t]{3}{*}{$\begin{array}{l}\text { NN163u } \\
u\end{array}$} & $\begin{array}{l}\text { Nakamura, Luis Hideo Vasconcelos } \\
\text { Utilização de web semântica para seleção de } \\
\text { informações no registro UDDI uma abordagem com } \\
\text { qualidade de serviço / Luis Hideo Vasconcelos } \\
\text { Nakamura; orientador Marcos José Santana. -- São } \\
\text { Carlos, } 2011 . \\
\quad 148 \text { p. }\end{array}$ \\
\hline & $\begin{array}{l}\text { Dissertação (Mestrado - Programa de Pós-Graduação en } \\
\text { Ciências de Computação e Matemática Computacional) -- } \\
\text { Instituto de Ciências Matemáticas e de Computação, } \\
\text { Universidade de São Paulo, } 2011 .\end{array}$ \\
\hline & $\begin{array}{l}\text { 1. Serviços Web. 2. Web Semântica. 3. Qualidade de } \\
\text { Serviços. 4. Avaliação de Desempenho. I. Santana, } \\
\text { Marcos José, orient. II. Título. }\end{array}$ \\
\hline
\end{tabular}


Agradeço inicialmente a Deus pela existência, por estar ao meu lado principalmente nos momentos mais difíceis, pelas oportunidades que colocou em meu caminho e por me conceder ânimo, força e coragem para superar os problemas e vencer mais uma etapa na minha vida. Agradeço a minha esposa, Ádria, que sempre me apoiou e incentivou durante esta jornada. Agradeço também a minha família, em especial aos meus pais, Pedro e Carmem, pelo carinho, apoio, paciência e compreensão.

Quero agradecer, em especial, ao meu orientador prof. Dr. Marcos Santana pela sua orientação e motivação como orientador. Pela sua dedicação e experiência transmitida como professor e pessoa. E também pelo o seu apoio em momentos de decisão. Quero agradecer também as professoras Regina e Sarita pelos conselhos e conhecimentos transmitidos. Ao professor Júlio Estrella, que também muito contribuiu com ideias e sugestões neste trabalho de mestrado e também pela sua prontidão, sempre pronto para participar, colaborar e contribuir. Agradeço aos demais professores do mestrado: Mônaco, Kalinka, Paulo Sérgio, Jo Ueyama e aos meus professores de graduação, em especial ao professor Marco Antônio, que muito contribuíram com a minha formação.

Agradeço também aos meus amigos que residem em São Carlos, Vagner Sousa, Ricardo Cezar (Amaral) e Valdir Sousa que me ajudaram no início do curso durante minha vinda à São Carlos. Aos meus amigos e colegas da CI\&T e IBM que muito me incentivaram nesta nova trilha que escolhi para minha vida.

Agradeço muito aos meus amigos do LaSDPC, pelo apoio, simpatia, hospitalidade e amizade. Um forte abraço a estes meus amigos: Bruno Guazzelli, Paulão (Ipuiuna), Bruno Tardiolle, Maycon Leone, Pedro Prado, Daniel Pigatto, Edwin Choquehuanca, Pedro Nobile, Bruno Faiçal, Douglas Rodrigues, Roni Apaza, João Paulo Orlando, Fabiano Texeira, Ricardo Nogueira, Lourenço Perreira, Alessandro Nakamuta, Renê Pinto, Dionísio Leite, Adriana Centurion, Thiago Caproni, Mário Machado e Júlio Estrella.

Agradeço a Universidade de São Paulo e a todos os seus funcionários, especialmente aos do ICMC. E também a todos aqueles que contribuíram de alguma forma para a realização bem sucedida deste projeto.

Quero agradecer também à FAPESP pelo apoio financeiro concedido para a realização deste trabalho. 

Ste projeto de mestrado aborda a utilização de recursos da Web Semântica na seleção de informações sobre Web Services no registro UDDI (Universal Description, Discovery, and Integration). Esse registro possui a limitação de apenas armazenar informações funcionais de Web Services. As informações não funcionais que incluem as informações de qualidade de serviço (QoS - Quality of Service) não são contempladas e dessa forma dificulta a escolha do melhor serviço pelos clientes. Neste projeto, a representação da base de conhecimento com informações sobre os provedores, clientes, acordos, serviços e a qualidade dos serviços prestados foi feita por meio de uma ontologia. Essa ontologia é utilizada pelo módulo UDOnt-Q (Universal Discovery with Ontology and $Q o S$ ) que foi projetado para servir de plataforma para algoritmos de busca e composição de serviços com qualidade. Embora a utilização de semântica possa ser empregada para a composição e automatização de serviços, o foco deste trabalho é a garantia de qualidade de serviço em Web Services. Os algoritmos desenvolvidos empregam recursos da Web Semântica para classificar e selecionar os Web Services adequados de acordo com as informações de qualidade que estão armazenados na ontologia. O módulo e os algoritmos foram submetidos a avaliações de desempenho que revelaram problemas de desempenho com relação a abordagem adotada durante o processo de inferência da ontologia. Tal processo é utilizado para a classificação das informações dos elementos presentes na ontologia. Contudo, uma vez que as informações foram inferidas, o processo de busca e seleção de serviços comprovou a viabilidade de utilização do módulo e de um dos seus algoritmos de seleção. 

7 His master project addresses the use of Semantic Web resources in the selection of information about Web Services in UDDI registry (Universal Description, Discovery, and Integration). This registry has the limitation of only storing functional information of Web Services. The nonfunctional information that includes the quality of service information (QoS - Quality of Service) is not covered and thus it is complicate to choose the best service for customers. In this project, the representation of the knowledge base with information about the providers, customers, agreements, services and quality of services has been made through an ontology. This ontology is used by the module UDOnt-Q (Universal Discovery with Ontology and QoS) that was designed to serve as a platform for search algorithms and composition of services with quality. Although the use of semantics can be adopted for the composition and automation of services, the focus of this work is to guarantee quality of service in Web Services. The developed algorithms employ Semantic Web resources to classify and select the appropriate Web Services according to the quality information that is stored in the ontology. The module and the algorithms have been subjected to performance evaluations that revealed performance problems in relation to the approach taken during the ontology inference process. This process is used for classification of information of the elements present in the ontology. However, since the information was inferred, the process of search and selection services proved the viability of using the module and one of its selection algorithms. 



\section{Resumo}

\section{Abstract}

Lista de Siglas

1 Introdução $\quad 1$

1.1 Considerações Iniciais $\ldots \ldots \ldots \ldots \ldots \ldots \ldots$

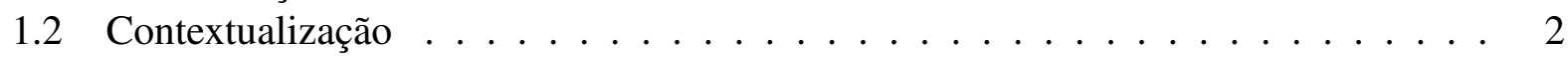

1.3 Trabalhos Relacionados . . . . . . . . . . . . . . . . . . . . . . . . . . . . . .

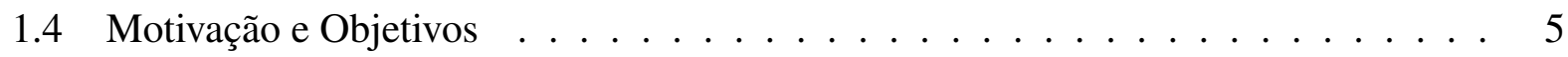

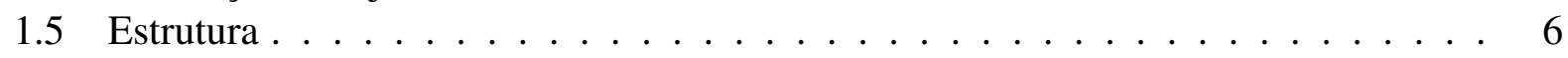

2 Web Services $\quad 7$

2.1 Considerações Iniciais $\ldots \ldots \ldots \ldots \ldots \ldots$

2.2 Arquitetura Orientada a Serviços $(\mathrm{SOA}) \ldots \ldots \ldots \ldots$

2.3 Padrões Fundamentais de Web Services . . . . . . . . . . . . . . . . . . . . . . . . . . . . . . . . .

2.4 Arquitetura de Web Services . . . . . . . . . . . . . . . . . . . . . . . 9

2.5 Qualidade de Serviço aplicada à Web Services . . . . . . . . . . . . . . . . . . . 11

2.6 Considerações Finais . . . . . . . . . . . . . . . . . . 12

3 Ontologia e Web Semântica $\quad 15$

3.1 Considerações Iniciais $\ldots \ldots \ldots \ldots \ldots \ldots \ldots$

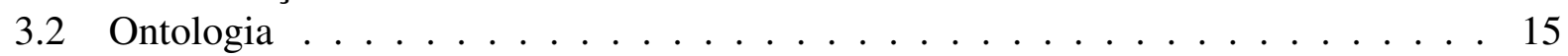

3.3 Web Semântica . . . . . . . . . . . . . . . . . . . . . 17

3.4 A OWL - Web Ontology Language . . . . . . . . . . . . . . . . . . . . . . 18

3.5 A OWL-S - Semantic Markup for Web Services . . . . . . . . . . . . . . . . . . 24

3.6 Ontologia, Web Semântica e Web Services . . . . . . . . . . . . . . . . . . . 25

3.7 Considerações Finais . . . . . . . . . . . . . . . . . 26

4 Ontologia do UDOnt-Q 29

4.1 Considerações Iniciais . . . . . . . . . . . . . . . . . . . . . . . 29

4.2 Desenvolvimento da Ontologia do UDOnt-Q . . . . . . . . . . . . . . . 30

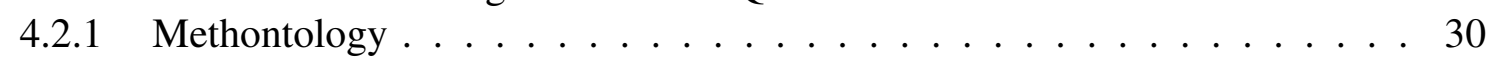


4.2.2 Metodologia de Noy e McGuinness . . . . . . . . . . . . . . . . . 32

4.2.3 Ferramentas para o desenvolvimento da Ontologia . . . . . . . . . . . 34

4.3 Elementos da Ontologia do UDOnt-Q . . . . . . . . . . . . . . . . . . 37

4.4 Considerações Finais . . . . . . . . . . . . . . . . . . . . . . . . . . 41

5 UDOnt-Q - Universal Discovery with Ontology and QoS 43

5.1 Considerações Iniciais ． . . . . . . . . . . . . . . . . . . . 43

5.2 Metodologia e Ferramentas . . . . . . . . . . . . . . . . . . . . . 43

5.2 .1 Metodologia Ágil (Agile) . . . . . . . . . . . . . . . . . . . . . . . . . . . . . 44

5.2 .2 Ferramentas . . . . . . . . . . . . . . . . . 44

5.3 Componentes e Estrutura . . . . . . . . . . . . . . . . . . . 48

5.3 .1 Componente Util . . . . . . . . . . . . . . . . . . . . . . . . . 49

5.3.2 Componente Command Components (CC) . . . . . . . . . . . . . . . 49

5.3 .3 Componente UDDI Components (UC) . . . . . . . . . . . . . . . 51

5.3.4 Componente Ontology Components (OC) . . . . . . . . . . . . . 51

5.3.5 Componente Common Information Shared (CIS) . . . . . . . . . . . . 53

5.3.6 Estrutura dos Componentes do UDOnt-Q . . . . . . . . . . . . . . 53

5.4 Considerações Finais . . . . . . . . . . . . . . . . . . . 56

6 Avaliação de Desempenho $\quad 57$

6.1 Considerações Iniciais . . . . . . . . . . . . . . . . . . . . . 57

6.2 Configuração do Ambiente de Testes . . . . . . . . . . . . . . . . . . . . . . 57

6.3 Planejamento de Experimentos . . . . . . . . . . . . . . . . . 60

6.4 Avaliação de Desempenho dos Experimentos . . . . . . . . . . . . . . 63

6.4.1 Cálculo da Influência dos Fatores . . . . . . . . . . . . . . . 66

6.5 Análise dos Resultados . . . . . . . . . . . . . . . . . . . . . 67

6.5.1 Análise dos Resultados das Ontologias . . . . . . . . . . . . . . . . 67

6.5.2 Análise dos Resultados do Módulo UDOnt-Q . . . . . . . . . . . . . . . . 67

6.6 Considerações Finais . . . . . . . . . . . . . . . . . . . . . . 97

7 Conclusões $\quad 99$

7.1 Conclusão Geral . . . . . . . . . . . . . . . . . . . . . . . . . . . . . . 99

7.2 Contribuições . . . . . . . . . . . . . . . . . . . 100

7.2.1 Produção Científica . . . . . . . . . . . . . . . . . . 101

7.3 Trabalhos Futuros . . . . . . . . . . . . . . . . 101

$\begin{array}{ll}\text { Anexos } & 111\end{array}$

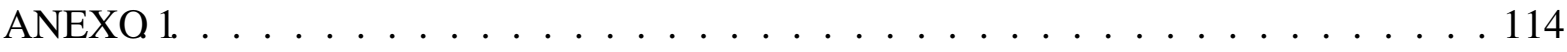

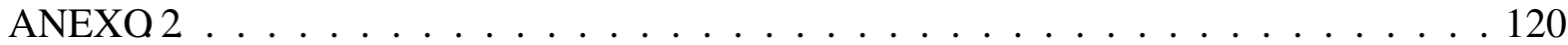

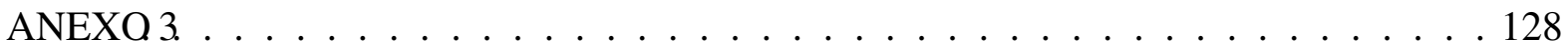

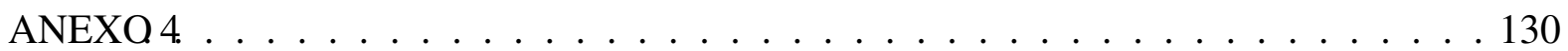




\section{Lista de Figuras}

2.1 Colaborações em uma arquitetura orientada a serviços (Traduzido de (Endrei et al., 2004)). . . . . . . . . . . . . . . . . . . . 8

2.2 Modelo básico de uma arquitetura orientada a serviços. . . . . . . . . . . . . . . 9

2.3 Pilha da Arquitetura de um Web Services. Adaptado de (W3C, 2004d). . . . . . . . 10

2.4 Web Service utilizando WSLA - Traduzido de (IBM, 2009). . . . . . . . . . . . . . 12

3.1 Exemplo de representação de Domínios. . . . . . . . . . . . . . . . . . . 17

3.2 Grafo representando os componentes básicos de um arquivo RDF. . . . . . . . . . 18

3.3 Arquitetura da Web Semântica (Berners-Lee, 2000) . . . . . . . . . . . . . . . . . 19

3.4 Arquitetura alternativa da Web Semântica (Antoniou e Harmelen, 2008) . . . . . . 19

3.5 Visão Taxonômica das classes: owl:Thing e owl:Nothing (Fisher et al., 2009) . . 20

3.6 Exemplos de Cabeçalho, Classe, Sub-Classe, Instância de Classe ou Indivíduo e Propriedade em OWL (Martimiano, 2006) . . . . . . . . . . . . . . . . . . . 22

3.7 Exemplo de propriedade Transitiva . . . . . . . . . . . . . . . . 23

3.8 Exemplo de propriedade Simétrica . . . . . . . . . . . . . . . . 23

3.9 Exemplo de propriedade Funcional . . . . . . . . . . . . . . . 23

3.10 Exemplo de propriedade Funcional . . . . . . . . . . . . . . . . 24

3.11 Topo do nível de uma ontologia de serviço. Traduzido de (Martin et al., 2004). . 24

4.1 Modelo abstrato da relação entre o cliente, módulo, algoritmos e a ontologia. . . . 29

4.2 Mapeamento da Hierarquia de Classes do UDOnt-Q. (Nakamura et al., 2011a) . . 35

4.3 Parte da aba de propriedades entre classes (Object Properties). . . . . . . . . . . . 35

4.4 Restrições de Equivalência da classe QoSGold. . . . . . . . . . . . . . . . . . 39

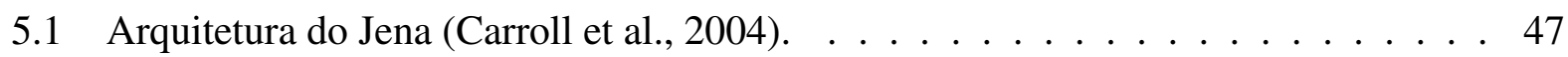

5.2 Componente Util. . . . . . . . . . . . . . . . . . . . . . . . . . . . . . . . . . . 49

5.3 Componente Command Components $(C C) \ldots \ldots \ldots$. . . . . . . . . 50

5.4 Sub-componente $Q o S$ Components $(Q C)$. . . . . . . . . . . . . . . . 50

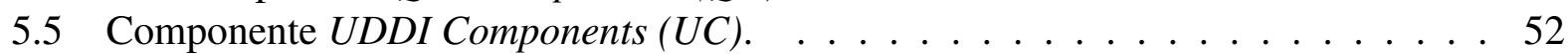

5.6 Componente Ontology Components (OC) . . . . . . . . . . . . . . . . 53

5.7 Componente Common Information Shared $(C I S)$. . . . . . . . . . . . . . . . . 53

5.8 Estrutura resumida do Módulo com os seus componentes (Nakamura et. al, 2011a). 54

6.1 Visão resumida dos elementos de software utilizados no ambiente de testes. . . . . 59

6.2 Visão resumida dos elementos de hardware utilizados. . . . . . . . . . . . . . . . 64 
6.3 Comportamento do processamento de Inferência da Ontologia com 30 Clientes e 300, 600, 900 e 1200 Serviços (PE-Ontologia).

6.4 Comportamento do processamento de Inferência da Ontologia com 200,400, 600 e 800 Pizzas (PE-Pizza).

6.5 Comportamento do processamento de Inferência da Ontologia com 300, 600, 900 e 1200 Serviços (PE-Ontologia) (Nakamura et al., 2011b).

6.6 Comportamento do processamento de Inferência da Ontologia com 200, 400, 600 e 800 Pizzas (PE-Pizza) (Nakamura et al., 2011b).

6.7 Gráfico em Linha de comparação do Comportamento do processamento de Inferência com 200, 400, 600 e 800 Pizzas (PE-Pizza) com um Comportamento Linear Hipotético (Nakamura et al., 2011b).

6.8 Tempo de Busca (TB) utilizando os algoritmos com 15 clientes (CJE-1). . . . . . 71

6.9 Tempo de Busca (TB) utilizando os algoritmos com 30 clientes (CJE-1). . . . . . 72

6.10 Tempo de Resposta para o Cliente (RTC) utilizando os algoritmos com 15 clientes (CJE-1).

6.11 Tempo de Resposta para o Cliente (RTC) utilizando os algoritmos com 30 clientes (CJE-1).

6.12 Porcentagem de Influência de cada fator no tempo de busca (TB) do módulo durante os experimentos (CJE-1).

6.13 Tempo de Busca (TB) utilizando os algoritmos com 15 clientes (CJE-2). . . . . . 75

6.14 Tempo de Busca (TB) utilizando os algoritmos com 30 clientes (CJE-2). . . . . . 75

6.15 Tempo de Resposta para o Cliente (RTC) utilizando os algoritmos com 15 clientes (CJE-2).

6.16 Tempo de Resposta para o Cliente (RTC) utilizando os algoritmos com 30 clientes (CJE-2).

6.17 Tempo de Busca (TB) utilizando os algoritmos com 30 clientes e todos os serviços (CJE-1 e 2) . . . . . . . . . . . . . . . . . . . . .

6.18 Porcentagem de Influência de cada fator no tempo de busca (TB) do módulo durante os experimentos (CJE-2).

6.19 Comparação no Tempo de Busca (TB) dos CJE-1 e CJE-3 utilizando os algoritmos com 30 clientes e 600 serviços.

6.20 Porcentagem de Influência de cada fator no tempo de busca (TB) do módulo durante os experimentos (CJE-3).

6.21 Comparação no Tempo de Busca (TB) dos CJE-2 e CJE-4 utilizando os algoritmos com 30 clientes e 1200 serviços.

6.22 Porcentagem de Influência de cada fator no tempo de busca (TB) do módulo durante os experimentos $(\mathrm{CJE}-4) . \ldots \ldots \ldots \ldots$. . . . . . . . . . 81

6.23 Tempo de Busca (TB) utilizando os algoritmos com 15 clientes (CJE-5). . . . . . 82

6.24 Comparação do Tempo de Busca (TB) entre o CJE-1 x CJE-5 utilizando 15 clientes, 300 serviços e o algoritmo OntAlgorithmSPARQL. . . . . . . . . . . . . 83

6.25 Tempo de Busca (TB) utilizando os algoritmos com 30 clientes (CJE-5). . . . . . . 84

6.26 Tempo de Resposta para o Cliente (RTC) utilizando os algoritmos com 15 clientes (CJE-5).

6.27 Tempo de Resposta para o Cliente (RTC) utilizando os algoritmos com 30 clientes (CJE-5).

6.28 Porcentagem de Influência de cada fator no tempo de busca (TB) do módulo durante os experimentos (CJE-5). 
6.29 Porcentagem da carga de processamento (CPU-Load) do módulo durante os experimentos (CJE-5). . . . . . . . . . . . . . . . . 87

6.30 Tempo de Busca (TB) utilizando os algoritmos com 15 clientes (CJE-6). . . . . . 88

6.31 Tempo de Busca (TB) utilizando os algoritmos com 30 clientes (CJE-6). . . . . . . 89

6.32 Tempo de Resposta para o Cliente (RTC) utilizando os algoritmos com 15 clientes (CJE-6). . . . . . . . . . . . . . . . . . . . . 90

6.33 Tempo de Resposta para o Cliente (RTC) utilizando os algoritmos com 30 clientes (CJE-6). . . . . . . . . . . . . . . . . . 90

6.34 Porcentagem de Influência de cada fator no tempo de busca (TB) do módulo durante os experimentos (CJE-6). . . . . . . . . . . . . . . . . . 91

6.35 Porcentagem da carga de processamento (CPU-Load) do módulo durante os ex-

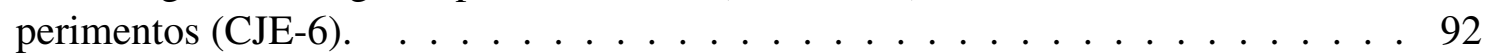

6.36 Tempo de Resposta para o Cliente (RTC) utilizando os algoritmos com 15 clientes (CJE-7). . . . . . . . . . . . . . . . . . . 93

6.37 Tempo de Resposta para o Cliente (RTC) utilizando os algoritmos com 30 clientes (CJE-7). . . . . . . . . . . . . . . . . . 93

6.38 Porcentagem de Influência de cada fator no tempo de resposta nos clientes (RTC) durante os experimentos (CJE-7). . . . . . . . . . . . . . . . . . . . 94

6.39 Tempo de Resposta para o Cliente (RTC) utilizando os algoritmos com 15 clientes

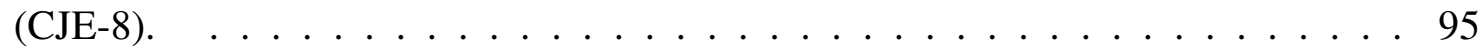

6.40 Tempo de Resposta para o Cliente (RTC) utilizando os algoritmos com 30 clientes

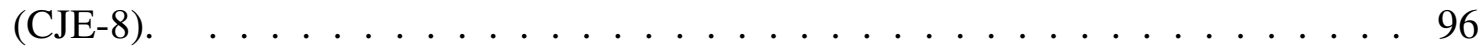

6.41 Porcentagem de Influência de cada fator no tempo de resposta nos clientes (RTC) durante os experimentos $(\mathrm{CJE}-8) . \ldots \ldots \ldots$. . . . . . . . . . . 97 



\section{Lista de Tabelas}

2.1 Atributos de QoS para Web Services (Lee e Shin, 2008) . . . . . . . . . . . . . 13

6.1 Elementos de Hardware . . . . . . . . . . . . . . . . . . . . . . 58

6.2 Elementos de Software . . . . . . . . . . . . . . . . . . . 58

6.3 Fator e níveis relativos ao PE-Ontologia . . . . . . . . . . . . . . 61

6.4 Fator e níveis relativos ao PE-Pizza . . . . . . . . . . . . . . . . . . 62

6.5 Fator e níveis relativos ao PE-Modulo . . . . . . . . . . . . . . . . . 62

6.6 Fatores Fixos e possíveis níveis relativos ao PE-Modulo . . . . . . . . . . . . . 63

6.7 Projeto Fatorial Completo para Avaliação de Desempenho do Módulo com 300 e 600 Serviços . . . . . . . . . . . . . . . . . . . . 65

6.8 Projeto Fatorial Completo para Avaliação de Desempenho do Módulo com 300 e 1200 Serviços . . . . . . . . . . . . . . . . . . 65

6.9 Conjuntos de Experimentos do PE-Modulo . . . . . . . . . . . . . . 65

6.10 Fatorial completo $2^{3}$ e Médias do Tempo de Busca (TB) do CJE-1 . . . . . . . . 73

6.11 Fatorial completo $2^{3}$ e Médias do Tempo de Busca (TB) do CJE-2 . . . . . . . . 78

6.12 Fatorial completo $2^{3}$ e Médias do Tempo de Busca (TB) do CJE-5 . . . . . . . . . 86

6.13 Fatorial completo $2^{3}$ e Médias do Tempo de Busca (TB) do CJE-6 . . . . . . . . 91

6.14 Fatorial completo $2^{3}$ e Médias do Tempo de Busca (TB) do CJE-7 . . . . . . . . . 94

6.15 Fatorial completo $2^{3}$ e Médias do Tempo de Busca (TB) do CJE-8 . . . . . . . . . 97 



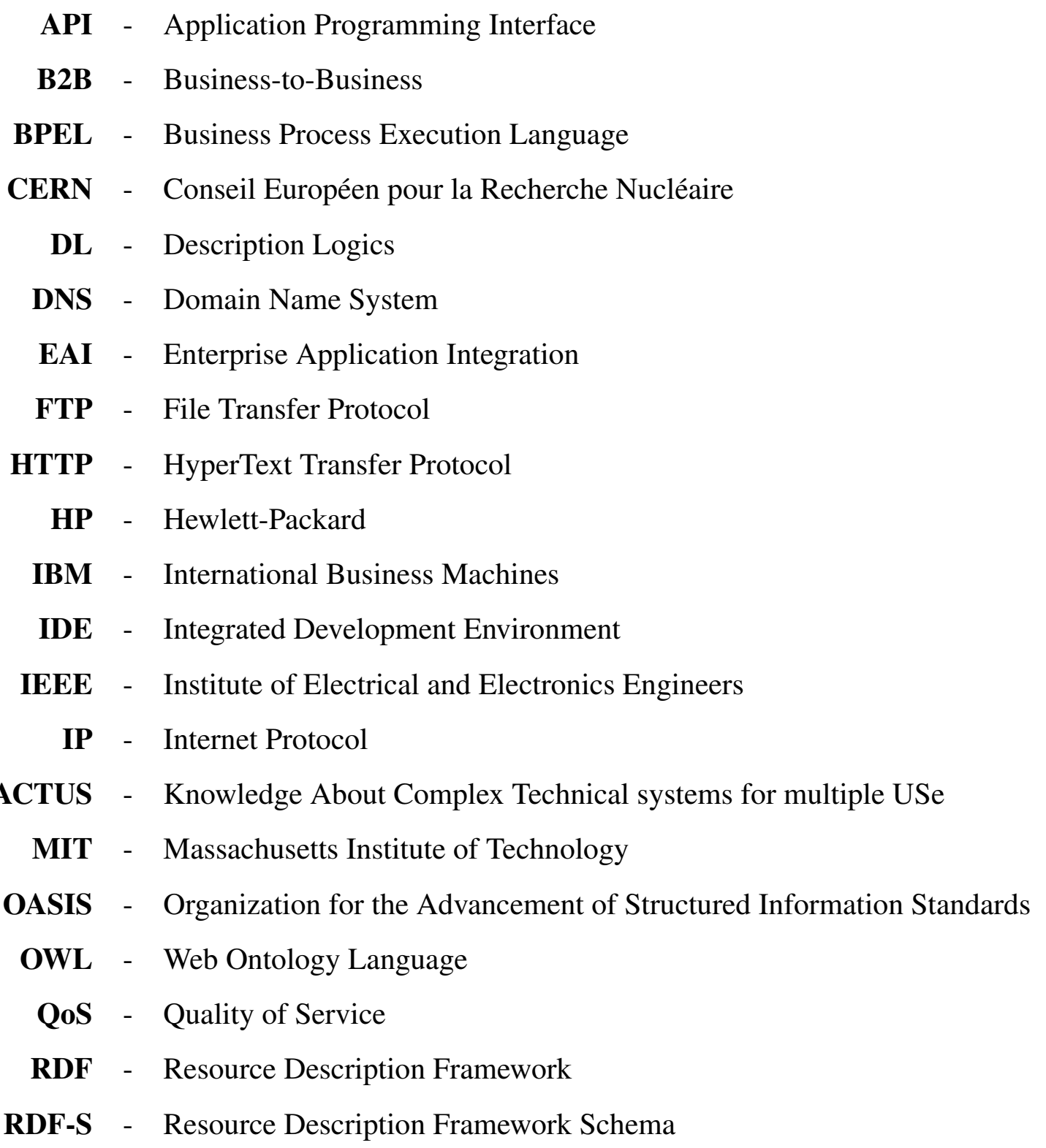




\author{
SaaS - Software-as-a-Service \\ SLA - Service Level Agreement \\ SMTP - Simple Mail Transfer Protocol \\ SOA - Service Oriented Architecture \\ SOAP - Simple Object Access Protocol \\ SPARQL - SPARQL Protocol and RDF Query Language \\ TI - Tecnologia da Informação \\ TOVE - TOronto Virtual Enterprise \\ UDDI - Universal, Discovery, Description and Integration \\ UDOnt-Q - Universal Discovery with Ontology and QoS \\ URI - Uniform Resource Identifier \\ W3C - World Wide Web Consortium \\ WS - Web Service \\ WSARCH - Web Services Architecture \\ WSDL - Web Services Description Language \\ WSLA - Web Service Level Agreement \\ XML - eXtensible Markup Language
}




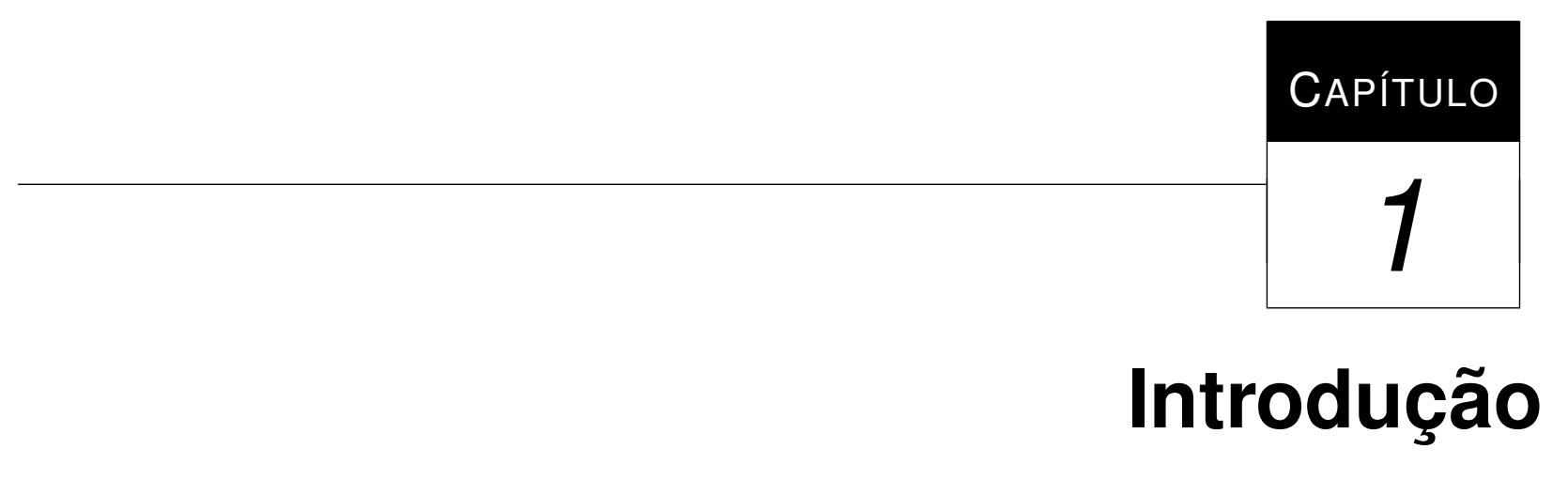

\subsection{Considerações Iniciais}

A World Wide Web (referida nesta dissertação por Web) foi concebida pelo físico Tim BernersLee em 1989, no Centro Europeu para Pesquisa Nuclear, o CERN (Lee e Fischetti, 1997). Anteriormente ao advento da $W e b$, a Internet era um reduto de pesquisadores ligados a universidades, governos e indústrias (Tanenbaum, 2003).

A Web facilitou o acesso à informação tornando-a mais disponível a várias pessoas ao redor do mundo. Nesse sentido as informações geograficamente distribuídas são acessadas de forma mais simples e ampla. O contexto que envolve a Web, de forma geral, vem passando por diversas modificações que vão desde a criação e alteração de protocolos até as aplicações que nela residem. A nova versão da Web foi batizada por Web 2.0 e engloba as aplicações de nova geração, com mais conteúdo dinâmico e provendo maior interatividade com os usuários (Murugesan, 2007).

Algumas das possibilidades para prover mais interação e dinamismo constituem as tarefas assíncronas e interação com outros sistemas ou aplicações. A Internet é formada por um conjunto de sistemas, aplicações e implementações diferentes e a interação entre esses sistemas não é uma tarefa simples, que requer a adoção de um padrão para a formalização de um protocolo. Os Web Services permitem a realização de interoperabilidade em nível de aplicação na interação máquina a máquina sobre uma comunicação em rede (Bhakti e Abdullah, 2010) e essa característica fez com que eles se tornassem uma solução atrativa para os problemas de integração entre sistemas na Internet.

Um Web Service é uma solução de software projetada para dar suporte a interações máquinapara-máquina, fornecendo uma interface descrita em um formato baseado em XML (eXtensible 
Markup Language), denominado WSDL (Web Services Description Language). Outros sistemas interagem com um Web Service da maneira prevista por sua descrição usando mensagens SOAP (Simple Object Access Protocol), que também são baseados em XML. Geralmente o protocolo utilizado para o transporte é o HTTP com uma serialização XML em conjunto com outros padrões relacionados à Web (W3C, 2004d). Para a publicação e descoberta dos provedores e de seus serviços é empregado o registro UDDI (Universal Description, Discovery, and Integration).

Os Web Services têm sido amplamente utilizados por vários segmentos, tanto na academia como em empresas, os quais têm investido em pesquisas com o propósito de melhorar a interoperabilidade, a eficiência, a segurança e o suporte à QoS (Quality of Service).

A ampla aplicabilidade de Web Services incentiva a pesquisa sobre a inclusão de qualidade de serviço (QoS). Algumas empresas têm trabalhado com o modelo SaaS (Software-as-a-Service), no qual a aplicação não é mais hospedada no host cliente, mas sim cedida como um serviço de modo que sua utilização pode ou não ser cobrada (Goh et al., 2007). Nesse sentido, além da qualidade de software, o cliente passará a exigir qualidade de serviço. É importante destacar que o cliente não é necessariamente o usuário final. Um cliente pode ser outra empresa ou outra aplicação e assim ser estendido a outros conceitos tais como: B2B (Business-to-Business) e EAI (Enterprise Application Integration).

Há na literatura várias propostas de aplicação de QoS em Web Services, onde algumas tentam utilizar semântica na busca de informações em Web Services. Alguns autores apresentam a utilização de ontologias com o objetivo de alcançar resultados mais positivos, como nos trabalhos de (Papaioannou et al., 2006), (Tran, 2008), (Maximilien e Singh, 2004), (Fakhfakh et al., 2008) e (Aklouf e Rezig, 2009). Segundo Tran (2008) a ontologia pode apoiar o descobrimento da informação de QoS com grandes detalhes. Ela também pode facilitar vários serviços participantes, os quais expressam suas ofertas e demandas de QoS em diferentes níveis de expectativa (Tran, 2008).

\subsection{Contextualização}

Os Web Services são baseados em uma arquitetura orientada a serviços - SOA (Service Oriented Architeture) que tem a vantagem de ser escalável e flexível. Os componentes de uma arquitetura orientada a serviços para Web Service, como o WSDL, o protocolo SOAP e o UDDI são baseados em XML, o que facilita a integração entre sistemas e aplicações diferentes, pois várias plataformas e linguagens de programação são capazes de trabalhar com XML. Um dos protocolos de transporte mais empregados para a transferência de mensagens SOAP é o HTTP (HyperText Transfer Protocol) que geralmente faz uso da porta 80, a qual na maioria dos firewalls não é bloqueada.

Todas essas vantagens fazem com que os Web Services se tornem um forte candidato para solucionar o problema da integração de sistemas e aplicações na Internet. Várias empresas têm investido e participado do desenvolvimento e de pesquisas em Web Services. A IBM, Hewlett- 
Packard - HP e a Microsoft, são alguns exemplos da indústria de TI (Tecnologia da Informação) que identificaram o potencial dos Web Services para resolver problemas de interoperabilidade.

Além da interoperabilidade, outra preocupação é com relação à qualidade de serviço prestada pelos provedores. Os clientes têm a opção de escolher serviços com a mesma funcionalidade em diferentes provedores. Quando o cliente conhece e tem contato com os provedores de serviços, ele pode se informar da qualidade de serviço (QoS) prestada e estabelecer acordos de níveis de serviços (SLA - Service Level Agreement). Até mesmo dentro de uma corporação podem existir serviços similares com mesma funcionalidade, porém com requisitos não funcionais (QoS) diferentes.

Quando o cliente não conhece os provedores ele pode consultar o registro UDDI (Universal Description, Discovery, and Integration) e obter informações sobre os serviços e provedores. Contudo, esse registro armazena apenas informações funcionais, portanto não há nenhuma informação de QoS que auxilie o cliente na escolha do serviço desejado (Tran e Tsuji, 2009). Além disso, diversos provedores podem compartilhar o mesmo registro UDDI com o objetivo de contemplar um maior número serviços que atendam aos mais diversificados tipos de clientes (os mais e os menos exigentes). A garantia do serviço prestado por esses provedores é estabelecida em acordos de níveis de serviços que buscam garantir a qualidade desejada de cada perfil de cliente. Este trabalho de mestrado está inserido dentro desse escopo, propondo o emprego de recursos da Web Semântica como solução para essa limitação do registro UDDI.

\subsection{Trabalhos Relacionados}

A ampla e iminente proliferação de Web Services despertou o interesse do meio acadêmico e da indústria em relação ao suporte à qualidade de serviço. Aplicações do tipo B2B, EAI e SaaS geralmente precisam disponibilizar pelo menos um mínimo de QoS. Algumas propostas ((Tavares e Westphall, 2006), (Xu et al., 2007), (Lee, 2008), (Ye et al., 2009), (Estrella et al., 2010), (Yuansheng et al., 2010), (Rajendran e Balasubramanie, 2010)) foram sugeridas nos últimos anos visando um melhor suporte à QoS, como a criação de novos componentes ${ }^{1}$ e arquiteturas.

Essas propostas são válidas, pois em uma arquitetura de Web Services são os provedores que disponibilizam os serviços e, consequentemente, são os responsáveis por garantir a qualidade adequada. Porém, mesmo que cada um dos provedores em particular garanta certo nível de QoS para os seus serviços, acontece muitas vezes o problema de os clientes não conhecerem, ainda, esses provedores e, neste caso, podem ser levados a iniciar uma pesquisa no registro UDDI. Contudo, tanto o padrão do registro UDDI como o padrão da WSDL falham na descoberta dinâmica de serviços baseada em qualidade de serviço, pois contam apenas com algumas operações funcionais de descoberta e publicação de Web Services e um conjunto de descrições sintáticas e estáticas da interface do serviço respectivamente (Kritikos e Plexousakis, 2007). Portanto, por padrão, eles não

\footnotetext{
${ }^{1}$ Geralmente próximo ou dentro do UDDI.
} 
contêm informações detalhadas de QoS para cada serviço. Nesse sentido, ainda surge a questão de como determinar quais serviços possuem um nível de QoS aceitável pelo cliente.

Com base nessa preocupação com a qualidade de serviços em Web Services, alguns outros trabalhos, tais como (Papaioannou et al., 2006), (Tran, 2008), (Maximilien e Singh, 2004), (Aklouf e Rezig, 2009), (Ma et al., 2010), (Baocai et al., 2010), (Wu e Guo, 2011) e (Tsai et al., 2011), abordam o emprego da semântica com ontologias para realizar a classificação dos serviços, tendo como base propriedades de QoS.

Em relação ao emprego de ontologias, no trabalho de Luo et. al. (2006) foi proposto um método de utilização da OWL-S com o registro UDDI para a obtenção de semântica (Luo et al., 2006). Nenhuma alteração no registro UDDI foi necessária, apenas a criação de módulos residentes nas máquinas clientes (client-side) que tiram proveito das capacidades da semântica. Contudo, não houve preocupação em relação à qualidade de serviço e, como utilizou a OWL-S, não apresentou uma nova ontologia e não houve uma avaliação do desempenho observado.

O trabalho de Baocai et. al. (2010) também considera a utilização da OWL-S, porém complementa a descoberta de serviços com auxílio de uma ontologia de QoS (QoS Ontology) utilizada por um framework para a descoberta automática dos serviços adequados. Porém, esse trabalho não considera os acordos de níveis de serviços (SLA) e não apresenta resultados de desempenho.

No trabalho de Fakhfakh et. al. (2008) é apresentada uma ontologia baseada no modelo SLA. Trata-se de um trabalho interessante que utiliza as obrigações acordadas em contratos, mas não apresenta resultados que possam ser avaliados em termos de desempenho e de eficiência na obtenção de QoS.

Os pesquisadores Wu e Guo (2011) propõem a utilização de agentes inteligentes providos pelo framework JADE ${ }^{2}$ (Java Agent DEvelopment Framework) na descoberta de Web Services. Apesar do emprego de agentes ser interessante, o artigo não descreve em detalhes a ontologia utilizada, os experimentos executados não detalham os elementos de software e não há registros dos elementos de hardware utilizados. Os resultados obtidos são interessantes e indicam que quanto maior o número de serviços, maior é o tempo de classificação mas, por outro lado, o tempo de descoberta é mais vantajoso utilizando-se essa abordem. Apesar de algumas semelhanças com os resultados deste trabalho de mestrado, comparações não podem ser feitas de forma expressiva em função da ausência de detalhes que não foram publicados.

A proposta de Tsai, Hwang e Tang (2011) consiste em uma abordagem híbrida entre o uso de ontologia e técnicas de recuperação de informações baseadas em textos, para descoberta automática de Web Services. Porém, nessa proposta as informações sobre a qualidade de serviço dos Web Services não são mencionadas.

De forma geral, apesar do embasamento teórico, poucos são os trabalhos relacionados que contemplam a descoberta de serviços baseados em QoS e ainda associam a parte de acordo de níveis de serviços (SLA). Além disso, nem todos esses trabalhos relatam os resultados de forma adequada,

\footnotetext{
${ }^{2}$ http://jade.tilab.com/
} 
alguns não apresentam informações de desempenho e outros não apresentam um desenvolvimento prático.

\subsection{Motivação e Objetivos}

Apesar de ser uma área que nos últimos anos está em destaque e em constante investigação e pesquisa, os Web Services ainda podem ser explorados em diversas vertentes, em função de sua natureza distribuída.

A maior aceitação e utilização de Web Services proporcionaram uma maior preocupação com o desempenho, confiabilidade e segurança dos mesmos. Porém todos estes pontos e alguns outros como interoperabilidade, tempo de resposta, custo, etc., podem ser englobados dentro do suporte à QoS para as aplicações. Embora várias pesquisas tenham sido desenvolvidas e diversos artigos tenham sido publicados, conforme apresentados na seção anterior, poucos propuseram a adoção de um ambiente próximo do real e a realização de testes que obtivessem resultados a serem analisados.

Nesse sentido, o objetivo deste projeto de mestrado é a utilização de Web Semântica na seleção de informações no registro UDDI, mas também vista ao suporte à QoS. Para realizar esta tarefa, a utilização de ontologia será adotada para auxiliar nas classificações de informações referentes ao contexto de Web Services com QoS. Dessa forma, ao invés do cliente requisitar informações ao registro UDDI, ele deve inicialmente consultar o módulo UDOnt-Q (desenvolvido neste trabalho) que realiza a busca por serviços baseando-se em determinados níveis ou parâmetros de QoS. O provedor de serviços deve manter atualizado os níveis ou parâmetros de QoS de seus serviços. Além disso, este trabalho também contempla a parte dos acordos de tipo SLA envolvidos, para determinar o nível de QoS de cada cliente. Esses contratos garantem a prestação do serviço, especificando claramente o custo, benefícios e punições do não cumprimento do acordo (Fakhfakh et al., 2008).

Alguns trabalhos relacionados, citados anteriormente, enfatizam apenas o desenvolvimento de ontologias para agregar QoS e não apresentam resultados que justifiquem ou não a sua utilização. Alguns trabalhos propõem uma implementação prática, mas não relatam detalhes de desenvolvimento e informações de desempenho. Neste trabalho foi implementado um módulo para servir de plataforma para algoritmos que empregam semântica para determinar quais serviços do UDDI são mais adequados às requisições de QoS dos clientes. Os algoritmos adotados utilizam informações presentes em uma ontologia, a qual representa o "mundo real" de uma arquitetura de Web Services com QoS. Além do desenvolvimento prático da ontologia, do módulo e de seus algoritmos, foi também realizada uma avaliação de desempenho criteriosa cujos resultados são apresentados no decorrer desta dissertação. 


\subsection{Estrutura}

Esta dissertação está organizada da seguinte forma:

- No Capítulo 2 são apresentadas informações sobre Web Services, seus conceitos, fundamentos e arquitetura. O conhecimento prévio sobre Web Services é necessário para o desenvolvimento deste trabalho, uma vez que a proposta é garantir qualidade para estes serviços. Além disso, as informações armazenadas, selecionadas e recuperadas do registro UDDI são referentes aos provedores de Web Services e também informações desses próprios serviços. Por fim, ainda neste capítulo, é destinado um espaço para a aplicação de qualidade de serviço (QoS) em Web Services.

- No Capítulo 3 são apresentados os conceitos de Web Semântica e Ontologia. O conhecimento de ambos os assuntos é indispensável para atingir o objetivo do projeto de pesquisa considerado nesta dissertação. A explicação do conceito de ontologia é realizada utilizandose um exemplo baseado em domínios. É apresentada uma revisão bibliográfica sobre Web Semântica, abordando os principais fundamentos e ferramentas utilizadas.

- No Capítulo 4 são apresentados o desenvolvimento e a estrutura da ontologia criada para servir de base de conhecimento para os mecanismos semânticos.

- No Capítulo 5 é caracterizada a estrutura geral do módulo UDOnt-Q, no qual serão discutidos os seus componentes. Também serão abordadas as implementações dos algoritmos de busca. O funcionamento da busca e seleção por parte do módulo e seus algoritmos são detalhados neste capítulo.

- No Capítulo 6 é discutida a avaliação de desempenho do módulo e seus algoritmos. Nesse capítulo são apresentadas algumas etapas que foram seguidas para a realização dos experimentos, como por exemplo; a configuração do ambiente, o planejamento dos experimentos e a análise dos resultados obtidos.

- O Capítulo 7 é reservado para as conclusões obtidas com o desenvolvimento deste trabalho. As principais contribuições geradas por este trabalho serão apresentadas em conjunto com sugestões para projetos futuros.

- Finalmente são apresentadas as Referências Bibliográficas utilizadas para a elaboração desta monografia.

No decorrer desta dissertação, o leitor irá notar que algumas imagens e tabelas foram traduzidas ou adaptadas para facilitar o entendimento do conteúdo. No entanto, algumas outras foram mantindas em seu formato e idioma (inglês) original de acordo como foram publicadas. 


\subsection{Considerações Iniciais}

Paralelamente ao avanço do potencial de processamento e do aumento da largura de banda, a Web evoluiu na direção de proporcionar um ambiente viável para a criação de aplicações robustas e complexas. Essa evolução estabeleceu um novo cenário composto de aplicações com jogos on-line, internet-banking, comércio eletrônico, páginas com conteúdo dinâmico e interação multimídia, entre outras. Dessa forma, a Web deixou de ser uma ferramenta apenas para a troca de arquivos e composta basicamente de páginas estáticas.

Com o aumento de aplicações na Internet, também aumentou a necessidade de comunicação entre elas. Contudo, o número de aplicações diferentes, construídas em ambientes operacionais distintos e escritas em linguagens heterogêneas tornou-se um obstáculo para tal interação. Os Web Services constituem uma solução apresentada para a interação entre aplicações. O objetivo deste capítulo é uma breve apresentação sobre os Web Services, abordando informações sobre a sua arquitetura, os padrões fundamentais e a qualidade de serviço relacionada.

\subsection{Arquitetura Orientada a Serviços (SOA)}

A Arquitetura Orientada a Serviços (SOA - Service-oriented Architecture) estabelece um modelo arquitetônico que busca utilizar serviços como principais meios para aprimorar a eficiência, a agilidade e a produtividade de uma empresa (Erl, 2009). SOA não é uma arquitetura concreta, não é um framework ou uma ferramenta. SOA pode ser tomada como um conceito ou um paradigma 
que, quando considerado, pode levar ao projeto de uma arquitetura concreta de software (Josuttis, 2008).

SOA propõe um estilo de arquitetura para a construção de sistemas de informação através de combinações de serviços (Komoda, 2006). A composição de serviços é um fator importante dentro de uma arquitetura orientada a serviços. Uma composição de serviço consiste em um conjunto ordenado de serviços (Erl, 2009), que melhora a flexibilidade e o reuso de serviços dentro da arquitetura orientada a serviços.

A Figura 2.1 ilustra as colaborações entre serviços de uma arquitetura SOA, onde as colaborações entre as entidades seguem o paradigma "procure, conecte e invoque" (" find, bind and invoke"), no qual o serviço consumidor realiza uma localização dinâmica, consultando o registro de serviços a fim de encontrar um que atenda os seus critérios. Assim, caso exista tal serviço, o registro fornece um contrato de interface e o endereço do serviço desejado. Por fim, o serviço consumidor conecta a um serviço provedor e consome o serviço. A descrição do serviço deve ser publicada no registro pelo serviço provedor, para que o serviço possa ser descoberto e acessado pelo serviço consumidor (Endrei et al., 2004).

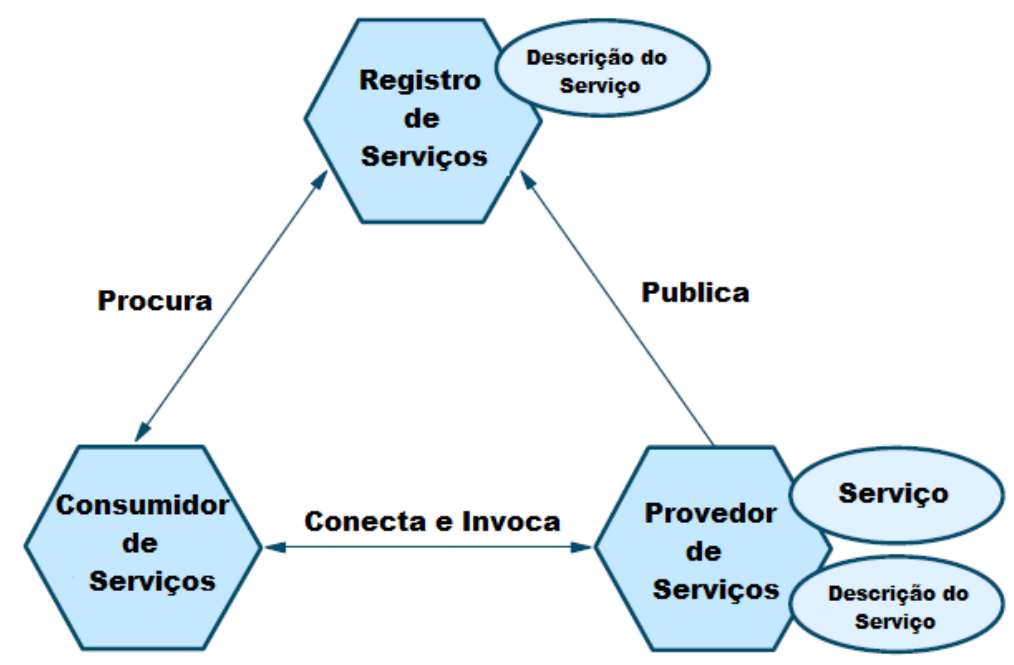

Figura 2.1: Colaborações em uma arquitetura orientada a serviços (Traduzido de (Endrei et al., 2004)).

\subsection{Padrões Fundamentais de Web Services}

Os Web Services são uma implementação da arquitetura SOA (Erradi e Maheshwari, 2005), a qual proporciona interoperabilidade, independência de ambiente operacional e linguagem de programação tornando a sua adoção mais atraente para a indústria de TI. Um modelo de Web Service possui interações entre três entidades, que são o provedor de serviços, o registro de serviços e o consumidor de serviços. A Figura 2.2 apresenta o modelo de uma SOA implementado para Web Services e seus componentes. 


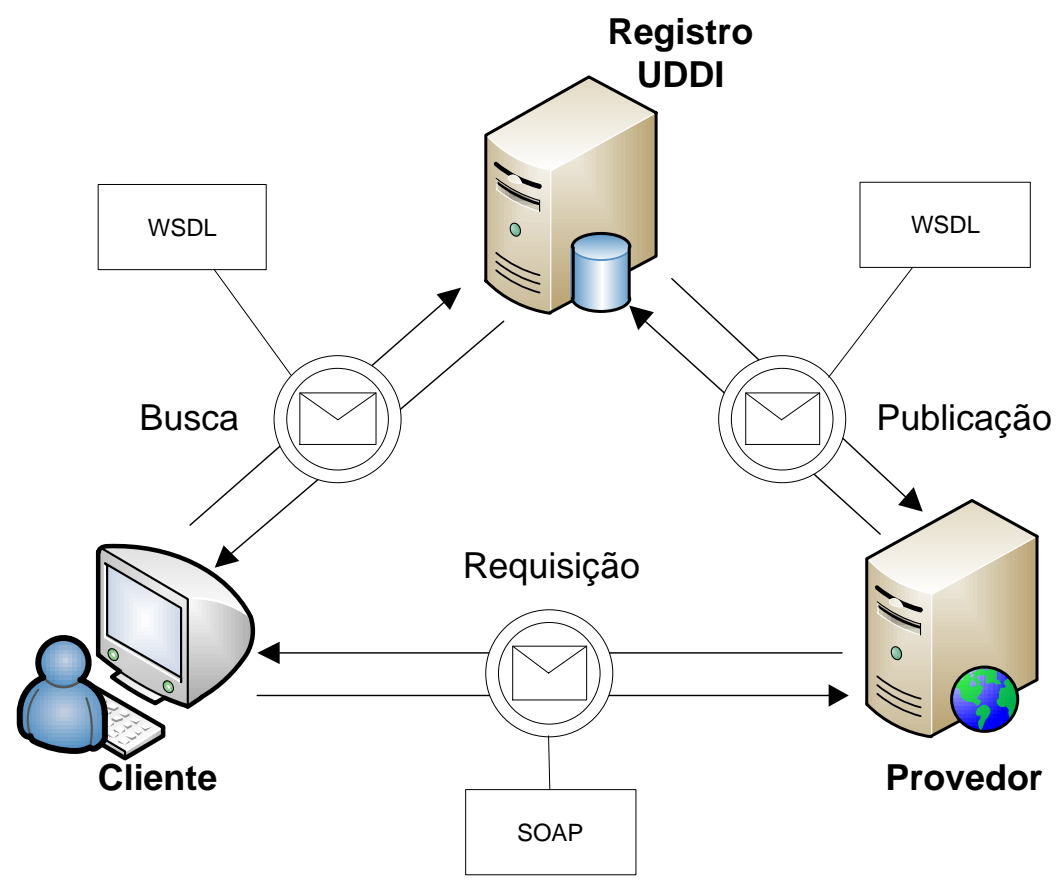

Figura 2.2: Modelo básico de uma arquitetura orientada a serviços.

Os Web Services são compostos de basicamente três padrões:

- Protocolo SOAP (Simple Object Access Protocol): é o protocolo utilizado para troca de mensagens entre Web Services, permitindo a comunicação de forma simples e independente de linguagem ou plataforma (Papazoglou e Georgakopoulos, 2003).

- Linguagem WSDL (Web Services Description Language): trata-se de uma linguagem utilizada para prover uma interface de descrição dos serviços de um Web Service. Ela especifica como o serviço deve ser acessado e quais os métodos disponíveis (Thomas et al., 2003).

- Registro UDDI (Universal Description, Discovery, and Integration): constitui um diretório (uma base de dados ou registro) que contém as descrições de Web Services, utilizado para localizar e publicar os serviços neste registro (Farkas e Charaf, 2003).

\subsection{Arquitetura de Web Services}

Padronização é um ponto importante dentro de uma arquitetura SOA, pois proporciona interoperabilidade, além de facilitar a implementação e o reuso. Esses padrões estão presentes na arquitetura de Web Services, a qual é composta por no mínimo um servidor que disponibiliza seus serviços na rede e por clientes que acessam esses serviços. Contudo, os agentes que irão utilizar os serviços necessitam de informações para localizar o servidor prestador do serviço e determinar os parâmetros exigidos. Para isso, o servidor deve publicar esses dados no registro UDDI que, 
por sua vez, pode conter informações de vários serviços de um mesmo servidor ou de servidores distintos. A WSDL é utilizada para publicação do serviço por parte do servidor e é encontrada na pesquisa por parte do agente consumidor. Uma vez que o agente consumidor possui as informações necessárias sobre o servidor e o serviço, que são encontradas na WSDL, ele realiza a requisição através do envio de uma mensagem encapsulada pelo protocolo SOAP.

Outro ponto a ser considerado é que componentes e sistemas legados também podem exercer o papel de consumidores de um Web Service, desde que sejam capazes de se comunicar utilizando os padrões dos Web Services (Erl, 2009). Utilizando-se os padrões estabelecidos é possível a comunicação entre aplicações de diferentes implementações, inclusive proprietárias. As aplicações podem ser escritas, por exemplo, em Java, .NET, PHP, entre outras.

A pilha da arquitetura de um Web Service é composta por várias camadas que vão desde o nível de transporte até a camada de processo, conforme mostra a Figura 2.3.

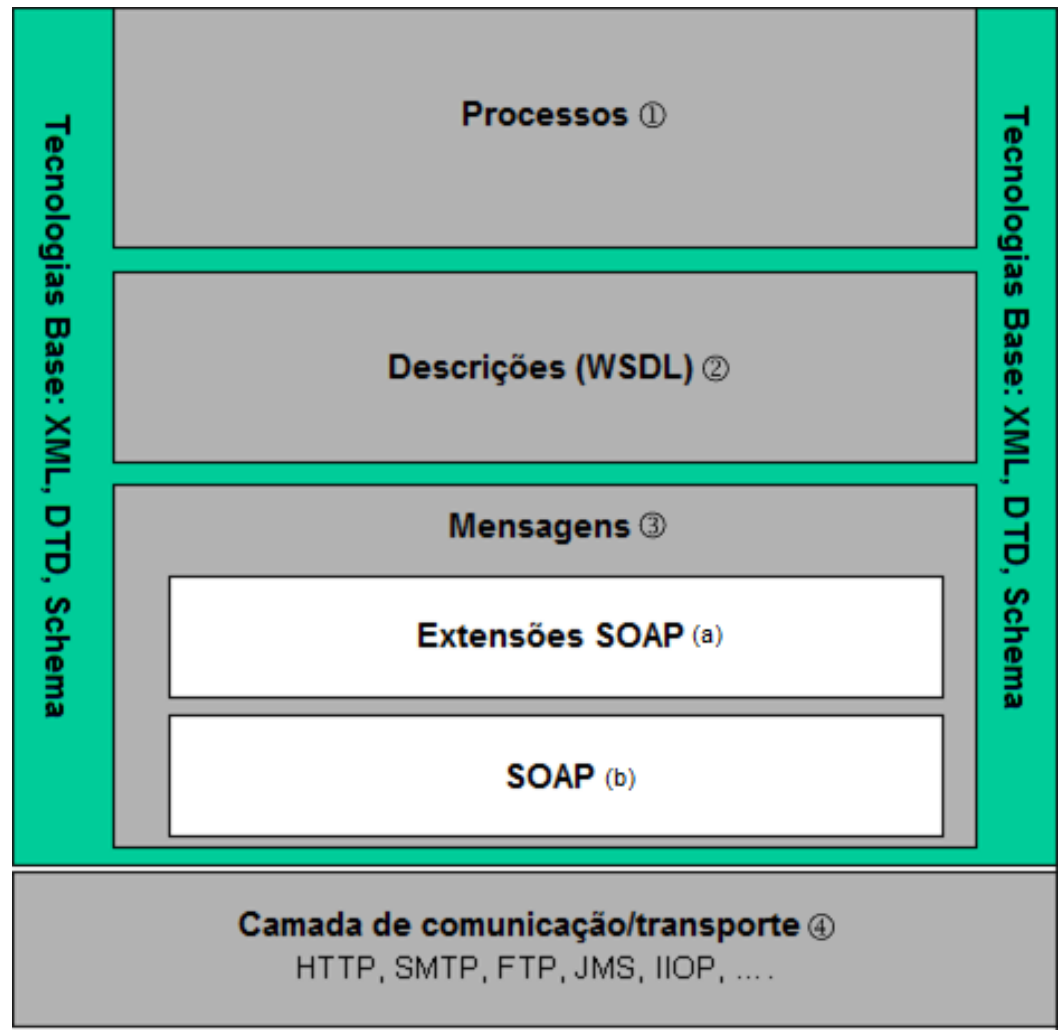

Figura 2.3: Pilha da Arquitetura de um Web Services. Adaptado de (W3C, 2004d).

Basicamente a pilha da arquitetura é formada pelas seguintes camadas:

1. Processos: Essa camada é responsável pelo gerenciamento e coordenação dos processos entre os serviços que compõe o Web Services. A linguagem BPEL (Business Process Execution Language) é utilizada na especificação dos processos de negócio e as suas relações com os Web Services.

2. Descrições: A camada de descrição contém a semântica formal para representar as mensagens que os Web Services podem entender, descrevendo as restrições de dados dentro das 
mensagens. Além disso, essa camada tem como responsabilidade definir a maneira como os Web Services são acessados (Tavares, 2009). Acrescenta-se ainda, que esta camada utiliza arquivos WSDL para a descrição de serviços, para o formato das mensagens, tipo de dados, protocolos de transporte, etc.

3. Mensagens: A camada de mensagem permite que a troca de mensagens seja feita em um ambiente descentralizado e distribuído de forma interoperável. (Toyohara, 2009). A mensagem pode ser serializada em um arquivo XML seguindo as especificações do protocolo SOAP.

- a. Extensões SOAP: São as extensões do protocolo SOAP. O protocolo SOAP pode ser modificado para suportar novas assinaturas e especificações dentro do seu padrão XML, como por exemplo: WS-Reliability, WS-Addressing, WS-Attachments, etc.

- b. SOAP: É o protocolo SOAP, baseado em XML.

4. Comunicação ou Transporte: especifica os protocolos de rede utilizados na troca de mensagens entre clientes e provedores de serviço. Dentre os principais protocolos, destacam-se o HTTP, SMTP, FTP, entre outros (Tavares, 2009).

Apesar de muitas pesquisas e estudos realizados desde sua criação, esta solução ainda está em fase de maturação. A W3C mantém um domínio de pesquisa voltado à Web Services denominado: "Web Services Activity"3 , em que uma parte dos trabalhos relacionados estão voltados à provisão de QoS e à Segurança.

A pesquisa por melhores resultados em qualidade de serviço deve ser contínua, uma vez que a indústria de TI sempre busca melhor atender aos seus clientes, desenvolvendo produtos com melhor qualidade e preço. Em uma rede como a Internet, a provisão de QoS em termos de desempenho, disponibilidade e segurança é essencial, e ao mesmo tempo não é uma tarefa trivial, em função da dinamicidade da Web.

\subsection{Qualidade de Serviço aplicada à Web Services}

Segundo Lee e Shin (2008) a qualidade de serviço (QoS) é um desafio crítico e importante devido à Internet ser uma rede dinâmica e de natureza imprevisível. Além disso, a QoS pode ser definida como uma combinação de várias qualidades ou propriedades de um serviço. Apesar das propriedades de qualidade de serviços não serem funcionais, elas são necessárias quando o serviço fornecido pelo provedor deve atender a certas qualificações exigidas pelos clientes.

Prover qualidade de serviço não é uma tarefa trivial, além disso, há a dúvida se o provedor de serviços está atuando para garantir o nível de qualidade de serviço exigido. Para solucionar este

\footnotetext{
${ }^{3}$ http://www.w3.org/2002/ws/
} 
problema, a IBM desenvolveu um framework denominado WSLA (Web Service Level Agreements) para especificação e monitoramento de acordos de níveis de serviços (SLA - Service Level Agreement) para Web Services (IBM, 2009). Análogo ao SLA, a WSLA é um acordo entre um provedor de serviço e um cliente que define as obrigações das partes envolvidas (Lee e Shin, 2008).

A WSLA complementa a definição do serviço estabelecida na WSDL. Enquanto a WSDL define a interface do Web Service, o WSLA define a forma de avaliar e mensurar as características de desempenho acordadas (em contrato, por exemplo, SLA). Tanto o cliente como o provedor de serviço executam o gerenciamento de monitoramento em suas infra-estruturas. Cada parte é responsável por mensurar as métricas de diversas fontes, por exemplo, o cliente deve mensurar se os valores dos parâmetros de qualidade de serviço foram alcançados (IBM, 2009). A Figura 2.4 exibe a estrutura de um Web Service utilizando WSLA.

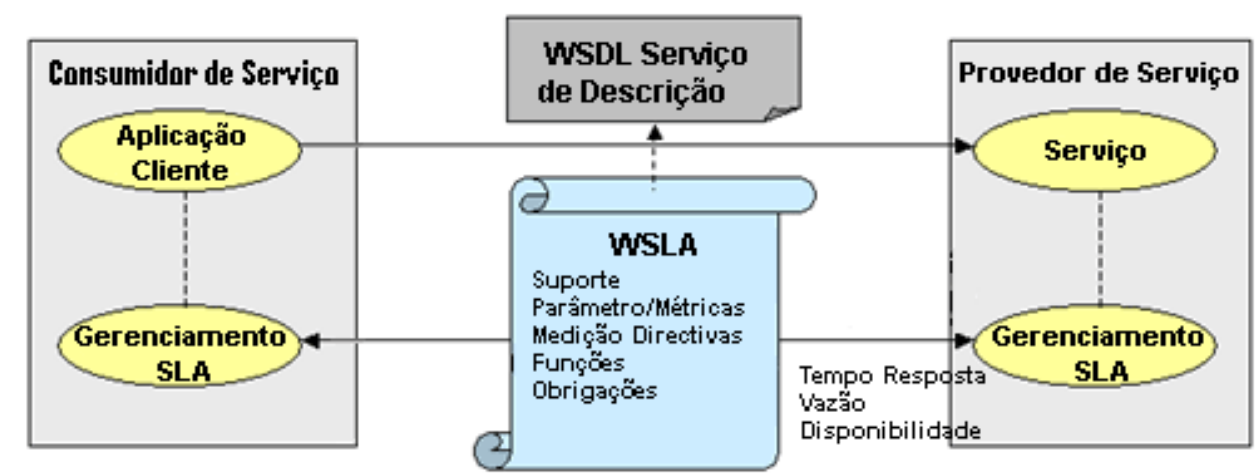

Figura 2.4: Web Service utilizando WSLA - Traduzido de (IBM, 2009).

A abordagem de utilizar ou não acordos, como o WSLA, fica a cargo das partes envolvidas. Para prover qualidade de serviço não é necessariamente preciso um acordo formal, o provedor pode desenvolver mecanismos que busquem garantir a qualidade de serviço e fornecê-los aos seus clientes com a intenção de oferecer um diferencial a mais que os seus concorrentes. Então, independente de acordos serem firmados, algumas diretrizes devem ser tomadas quando se pretende fornecer qualidade de serviço; uma delas é definir quais atributos (ou propriedades) de QoS serão atendidos e definir os valores (ou níveis) desses atributos. Alguns dos principais atributos de QoS para Web Services citados por Lee e Shin (2008) são apresentados na Tabela 2.1:

\subsection{Considerações Finais}

Neste capítulo, foram apresentadas informações sobre Web Services, seus conceitos, fundamentos e arquitetura. Os Web Services são implementações da arquitetura orientada a serviços (SOA) que ganharam destaque nos últimos anos. Devido à rápida "popularização" dos Web Services na indústria de TI e no meio acadêmico, notou-se a necessidade de garantir a qualidade dos Web Services. Alguns trabalhos relacionados foram citados, argumentando essa necessidade. Um exemplo de solução para garantia de QoS, o WSLA, foi comentado e finalmente alguns dos 
Tabela 2.1: Atributos de QoS para Web Services (Lee e Shin, 2008)

\begin{tabular}{|c|c|}
\hline Atributo de QoS & Descrição \\
\hline Tempo de Resposta & Tempo que um serviço leva para completar uma tarefa. \\
\hline Latência & Tempo necessário para iniciar um serviço requisitado. \\
\hline Vazão & Taxa de processamento de um serviço para atender a solicitação. \\
\hline Escalabilidade & É o aumento da vazão em um dado intervalo de tempo. \\
\hline Disponibilidade & Consiste em o Web Service estar presente ou pronto para \\
utilização.
\end{tabular}

parâmetros (atributos ou propriedades) de QoS para Web Services foram listados. Alguns desses atributos serão considerados neste projeto, pois estão presentes na literatura e são amplamente utilizados pelas empresas de TI. 



\section{Ontologia e Web Semântica}

\subsection{Considerações Iniciais}

Este capítulo aborda dois conceitos importantes para o desenvolvimento desta dissertação de mestrado que são Ontologia e Web Semântica aplicados aos Web Services. A Web Semântica está sendo explorada nos últimos anos, juntamente com o emprego de ontologias, para definir um vocabulário mais rico e aperfeiçoar a eficiência de mecanismos semânticos.

Neste capítulo são apresentados alguns conceitos sobre Ontologia (Seção 3.2) e Web Semântica (Seções 3.3 e 3.4), aplicados no contexto de Web Services (Seção 3.5). Na Seção 3.6, é feita uma associação de todos os conceitos e, finalmente, na Seção 3.7 são apresentadas as considerações finais deste capítulo.

\subsection{Ontologia}

Ontologia é um conceito que teve origem nos estudos filosóficos. O termo ontologia é a composição de dois ramos: onto (ser) e logia (estudo); ou seja, a ontologia pode ser considerada o estudo do ser em uma ênfase existencial.

O termo ontologia, que ficou restrito à Filosofia durante anos (Carase, 2005), começou a ser empregado na ciência da computação a partir do final dos anos 70 (Martimiano, 2006). Com base no conceito do estudo do ser, as ontologias facilitam a descrição dos seres, estudando suas características, capacidades, relacionamentos, etc. Nesse contexto, pode-se notar quão importante as ontologias são para a criação de semânticas. 
As ontologias podem então ser utilizadas para a criação de um vocabulário baseado em um determinado domínio de conhecimento, o qual pode ser compartilhado e re-utilizado. Este vocabulário pode ser fornecido de forma explícita e com um grau de formalidade suficiente, o qual permite o seu processamento por agentes computacionais. O grau de formalidade pode variar, e segundo Uschold e Gruninger (1996), esse grau de formalidade pode ter quatro níveis (Uschold e Gruninger, 1996):

- Altamente Informal: utiliza livremente a linguagem natural para expressar o vocabulário.

- Semi-Informal: utiliza a linguagem natural, porém de uma forma restrita e estruturada com bastante clareza, reduzindo assim a ambiguidade.

- Semi-formal: utiliza uma linguagem artificial definida formalmente para expressar o vocabulário.

- Rigorosamente formal: os termos são definidos minuciosamente através de semânticas formais, teoremas e provas que garantam a solidez e completude.

A utilização de ontologias em ciências de computação geralmente é limitada em domínios. Esses domínios de conhecimento podem ser compartilhados e auxiliam na interoperabilidade semântica. Segundo Noy e McGuinness (2001), uma ontologia é uma “descrição formal e explícita dos conceitos de um domínio de conhecimento, das suas propriedades (atributos e relacionamentos) e restrições" (Noy e McGuinness, 2001). A Figura 3.1 demostra uma divisão de domínios, na qual existe o domínio "Hardware", que possui os subdomínios "Periféricos" e "Computadores", que por sua vez também contêm outros subdomínios; "Disp Entrada", "Disp Saída", "Pessoal" e "Grande Porte".

Esses domínios foram descritos em uma forma que segue a visão top-down, podendo também serem descritos na ordem inversa, ou seja, na visão bottom-up. Esses dois tipos de visões são interessantes no momento de se modelar uma ontologia. A combinação de ambos (top-down e bottom-up) também pode ser explorada. Além disso, na mesma Figura 3.1 é possível observar o domínio "Informática", o qual está relacionado com o domínio "Hardware". É importante destacar que os domínios também podem ter propriedades, como é o caso do domínio "Hardware" com as seguintes propriedades: Chips, Circuitos, Transistores, Fios, Sensores, Etc.

Para desenvolver uma ontologia de forma organizada é preciso uma metodologia. Há diversas metodologias que podem ser empregadas tais como: Methontology (Lopez et al., 1997), metodologia de Noy e McGuinness (2001), KACTUS (Knowledge About Complex Technical systems for multiple USe) (Bernaras et al., 1996), TOVE (TOronto Virtual Enterprise) (Gruninger e Fox, 1995), etc. No desenvolvimento deste trabalho de mestrado está sendo considerada a Methontology (Lopez et al., 1997) em paralelo com a metodologia de Noy e McGuinness (2001) cujo título é "Ontology Development 101: A Guide to Creating Your First Ontology". A escolha por essas metodologias é justificada no capítulo 4, onde se descreve o desenvolvimento da ontologia. 


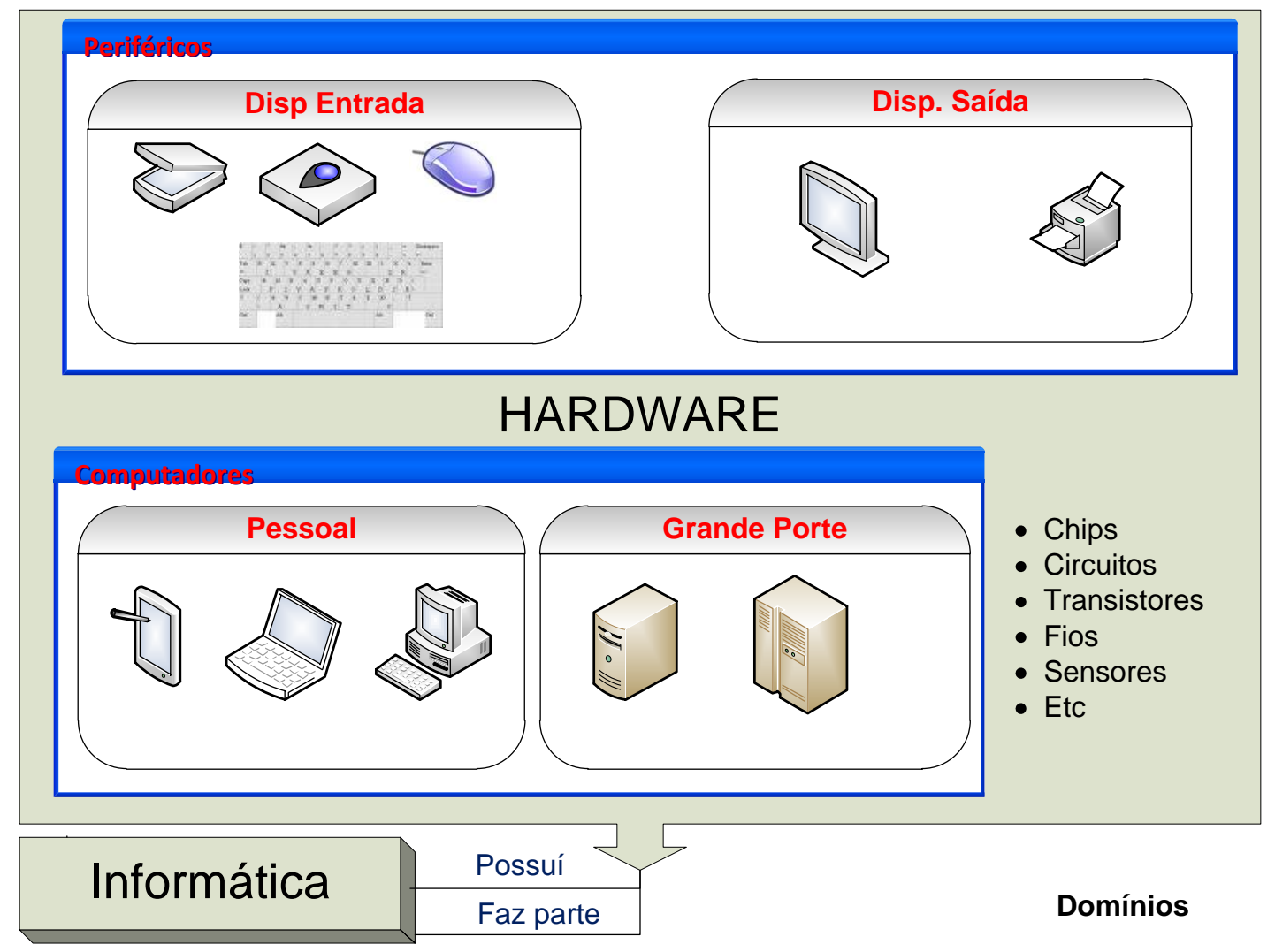

Figura 3.1: Exemplo de representação de Domínios.

\subsection{Web Semântica}

A Web é uma das maiores fontes de informação existente atualmente, sendo, portanto, importante interpretar, selecionar (ou classificar) e analisar o seu conteúdo. Para que máquinas (computadores) consigam tamanha proeza é necessário que elas sejam capazes de não apenas ler as informações, mas compreender do que se trata.

A W3C (World Wide Web Consortium) tem incentivado as pesquisas em Web Semântica. Ela propõe uma visão pioneira, na qual a informação pode ser entregue de forma explícita, proporcionando que as máquinas consigam processar e integrar informações de uma maneira mais fácil sugerindo assim a utilização da Web Semântica (W3C, 2004c).

Para representar as informações na Web, a W3C desenvolveu um framework denominado RDF (Resource Description Framework), que permite adicionar informações sobre determinado recurso na Web (uma página, por exemplo). Dessa forma, é possível adquirir não apenas o conteúdo, mas também informações capazes de atribuir uma semântica àquele recurso (W3C, 2004c).

A estrutura de qualquer expressão em RDF é uma coleção baseada em triplas compostas por Sujeito, Predicado e Objeto. Um conjunto de triplas é denominado grafo RDF (W3C, 2004c). A Figura 3.2 exibe os três componentes básicos de um arquivo RDF, no qual a Página 1 é o Sujeito, a Página 2 é o Objeto e uma propriedade em comum (creator - criador) é o Predicado. 


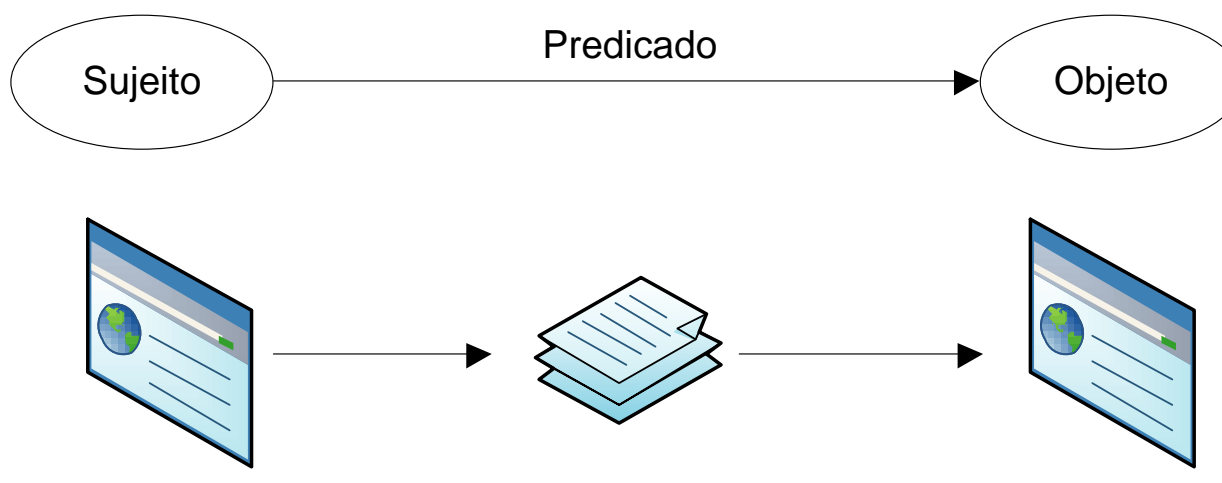

\section{Página 1 Propriedade em Comum Página 2 $<$ dc:creator $>$ Luis Nakamura</dc:creator $>$}

Figura 3.2: Grafo representando os componentes básicos de um arquivo RDF.

O RDF é baseado em XML, e permite a representação de relações entre objetos, possuindo melhores funcionalidades para representar semântica. Os documentos RDF são compostos por declarações sobre os recursos ou objetos representados, porém cada declaração é composta pelo objeto, por uma propriedade e o valor dessa propriedade, que pode ser até mesmo outro objeto. Da mesma forma que o XML, o RDF também permite que agentes computacionais interpretem o significado dos dados por meio das tags associadas a ele (Martimiano, 2006).

O RDF não provê nenhum mecanismo para descrever as propriedades, tão pouco descrever as relações entre estas propriedades e outros recursos. O responsável por obter essa respectiva semântica é o Esquema RDF (RDF Schema), que define classes e propriedades que podem ser usadas para descrever as classes, propriedades e outros recursos (W3C, 2004b).

Além disso, pesquisadores notaram algumas limitações no RDF e RDFS (RDF Schema). Em Heflin (2001), é relatado que o RDF não possui um mecanismo para uma definição geral de axiomas (Heflin, 2001). Segundo Broeksta (2001), as primitivas definidas no RDF Schema não provêm uma semântica formal, e a expressividade dessas primitivas não é suficiente para o desenvolvimento de modelos ontológicos puros e de raciocínio (Broekstra et al., 2001). Dessa forma, foi necessária a criação da linguagem OWL (Web Ontology Language), que estende o conceito do RDF para criação de ontologias.

\subsection{A OWL - Web Ontology Language}

A OWL (Web Ontology Language) é uma linguagem projetada para uso em aplicações que precisam processar o conteúdo da informação e não apenas apresentar essas informações para os seres humanos. A OWL proporciona uma maior interpretabilidade do conteúdo da Web pelas máquinas (computadores) do que com XML, RDF e RDF Schema, disponibilizando um vocabulário adicional com uma semântica formal (W3C, 2004c). 
Com a OWL é possível adicionar vocabulários mais ricos para descrever as classes, os relacionamentos entre classes, comparação entre classes, restrições de cardinalidade e as características das propriedades (Martimiano, 2006).

A linguagem OWL fornece três subdivisões, cada uma para uso específico (W3C, 2004c):

- OWL Lite: trata-se de uma definição simples de hierarquia de classes e com poucas restrições de propriedades.

- OWL DL (Description Logics): trata-se de uma definição de OWL que oferece funcionalidades expressivas. Permite a utilização dos construtores da OWL, porém possui algumas restrições, mantendo a completude computacional (capacidade de ser processada) e a tomada de decisão em um tempo finito. A OWL DL é um meio termo entre a OWL Lite e a OWL Full, e está baseada em lógicas de descrição (DL). Muitos adotam essa subdivisão como padrão para desenvolvimento.

- OWL Full: é a OWL mais completa, e apresenta todos os construtores disponíveis sem restrição e é independente da sintaxe do RDF. Por ser muito abrangente, não há garantia que um software seja capaz de interpretar a ontologia criada com a OWL Full.

Tim Berners-Lee apresentou em 2000 uma arquitetura em pilha (também denominada de camadas do bolo - layer cake), representada na Figura 3.3, para explicar os diferentes protocolos e os desafios da Web Semântica (Berners-Lee, 2000). Segundo Antoniou e Harmelen (2008), a camada de ontologia é instanciada com duas alternativas: a OWL e uma linguagem baseada em regras (Rules) (Antoniou e Harmelen, 2008). A Figura 3.4 mostra um modelo atualizado de pilha da Web Semântica no qual as camadas "abstratas" propostas por Berners-Lee são representadas por algumas das atuais linguagens utilizadas na área.

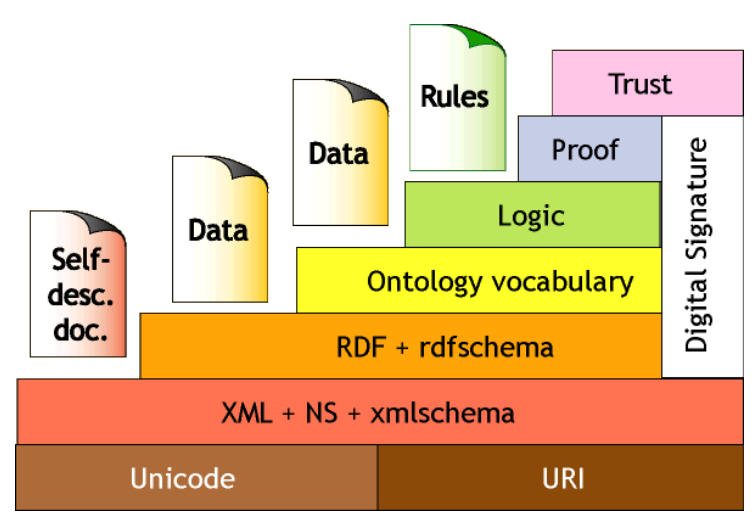

Figura 3.3: Arquitetura da Web Semântica (Berners-Lee, 2000)

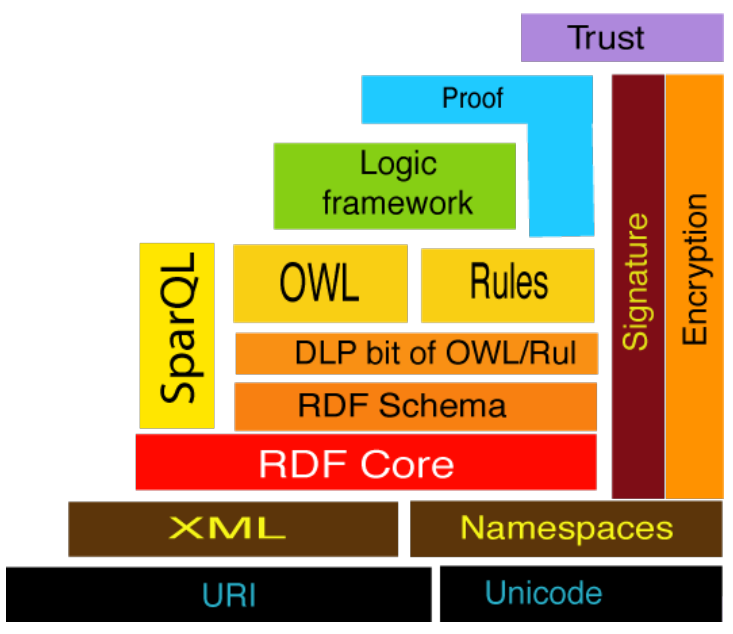

Figura 3.4: Arquitetura alternativa da Web Semântica (Antoniou e Harmelen, 2008)

Na OWL, há duas classes fundamentais: owl:Thing e owl:Nothing. A owl:Thing representa a classe de todos os indivíduos e todo recurso que é uma instância de uma classe é implicitamente 
um membro da owl:Thing. A owl:Nothing representa uma classe vazia, uma classe que não possui membros. A classe owl:Thing é a classe mais geral, enquanto a owl:Nothing é a classe mais específica possível; a Figura 3.5 demostra uma visão taxonômica dessas classes (Fisher et al., 2009).

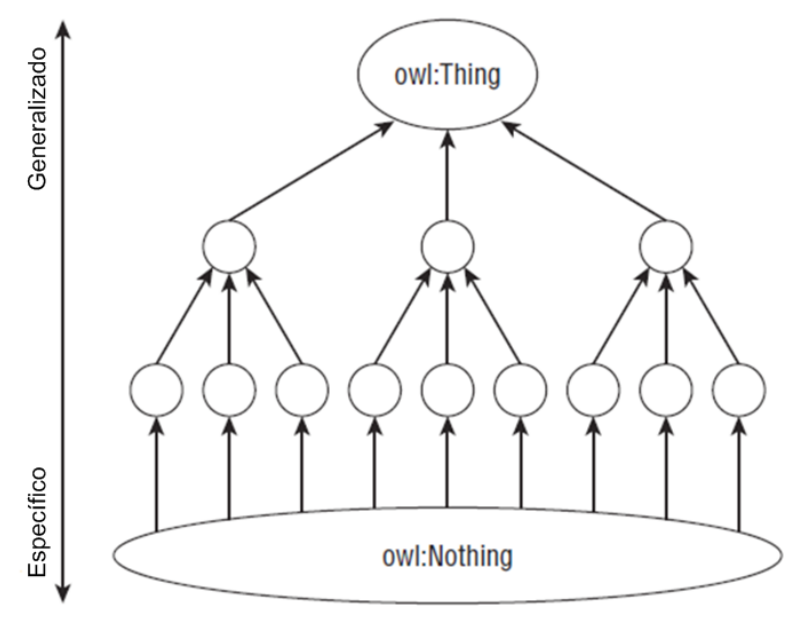

Figura 3.5: Visão Taxonômica das classes: owl:Thing e owl:Nothing (Fisher et al., 2009)

Dessa forma, todas as classes de uma ontologia em OWL são subclasses da classe owl:Thing. As classes owl:Class e $r d f$ s: $s u b C l a s s O f$ são utilizadas para a criação de hierarquia entre classes na OWL. A OWL também fornece algumas formas de descrever propriedades (adicionando semântica). Existem diversas propriedades disponíveis na linguagem OWL das quais pode-se destacar (Antoniou e Harmelen, 2008):

\section{$\diamond$ Propriedades de Elementos:}

- Propriedade de Objeto (owl:ObjectProperty): Propriedade que relaciona objetos à outros objetos.

- Propriedade de Tipo de Dados (owl:DatatypeProperty): Propriedade que relaciona objetos a tipos de dados. A OWL utiliza os tipos de dados prescritos no XML Schema.

- Propriedade Inversa (owl:inverseOf): Propriedade que determina que uma determinada propriedade é inversa (contrária) a outra propriedade existente.

- Propriedade Equivalente (owl:equivalentProperty): Propriedade que define que uma determinada propriedade é equivalente a outra propriedade existente.

$\diamond$ Propriedades de Restrições (owl:Restriction): Uma restrição deve ser relacionada a uma propriedade existente, essa propriedade é definida no atributo owl:onProperty:

- Todos os Valores De (owl:allValuesFrom): Propriedade utilizada para especificar a classe com os possíveis valores de uma propriedade (especificada em owl:onProperty). 
- Tenha o Valor (owl:hasValue): Propriedade que especifica o valor que a propriedade (especificada em owl:onProperty) deve ter.

- Algum Valor De (owl:someValuesFrom): Propriedade utilizada para especificar a classe e a ocorrência de pelo menos um dos valores dentre os possíveis de uma propriedade.

- Cardinalidade Mínima (owl:minCardinality): Indica o valor mínimo existente em um relacionamento. Pode utilizar datatypes para expressar restrições, por exemplo: "nonNegativeInteger".

- Cardinalidade Máxima (owl:maxCardinality): Indica o valor máximo existente em um relacionamento. Também pode utilizar datatypes para expressar restrições.

\section{$\diamond$ Propriedades Especiais:}

- Propriedade Transitiva (owl:TransitiveProperty): Define que uma propriedade é transitiva. Por exemplo, se uma propriedade $p$ é dita transitiva e relaciona a classe A com a classe $\mathrm{B}(\mathrm{A} p \mathrm{~B})$ e também relaciona a classe $\mathrm{B}$ com a classe $\mathrm{C}$ (B $p \mathrm{C}$ ), e então, sendo transitiva, ela garante a relação entre A e C (A p C) (Fisher et al., 2009).

- Propriedade Simétrica (owl:SymmetricProperty): Define a simetria entre as classes. Por exemplo, se uma propriedade $p$ é dita simétrica e relaciona as classes A e B (A $p$ B) então ela também garante a simetria de B e A (B $p$ A) (Fisher et al., 2009).

- Propriedade Funcional (owl:FunctionalProperty): Define uma propriedade que tem ao menos um valor para cada objeto (Antoniou e Harmelen, 2008). Uma propriedade funcional pode ser associada a apenas um único valor para um indivíduo em particular. Por exemplo, a data de nascimento; um indivíduo pode ter apenas uma data de nascimento.

- Propriedade Funcional Inversa (owl:InverseFunctionalProperty): Define uma propriedade para a qual dois diferentes objetos não podem ter o mesmo valor (Antoniou e Harmelen, 2008). Um exemplo é uma propriedade "CPF”, em que diferentes indivíduos não podem ter o mesmo valor de CPF.

Um exemplo de Classe, Sub-Classe, Instância da Classe ou Indivíduo e Propriedade em um arquivo de ontologia OWL são apresentados na Figura 3.6. No início do arquivo, tipicamente, há um cabeçalho que define os espaços de nomes XML (namespaces XML-xmlns) que especificam os vocabulários utilizados (Smith et al., 2004).

Na Figura 3.6, as duas primeiras linhas (11 e 12) do cabeçalho referem-se ao namespace do vocabulário da ontologia corrente ("wine”), sendo a primeira linha (11) o namespace geral da ontologia corrente, que implica que qualquer nome declarado nesse espaço pertence a ele, a menos que esse nome tenha uma referência para outro espaço de nomes. Já a segunda linha (12) define um prefixo ("vin”) que é associado ao namespace da ontologia para referenciar definições do vocabulário (Martimiano, 2006). A terceira linha (13) associa o namespace corrente com o URI do 


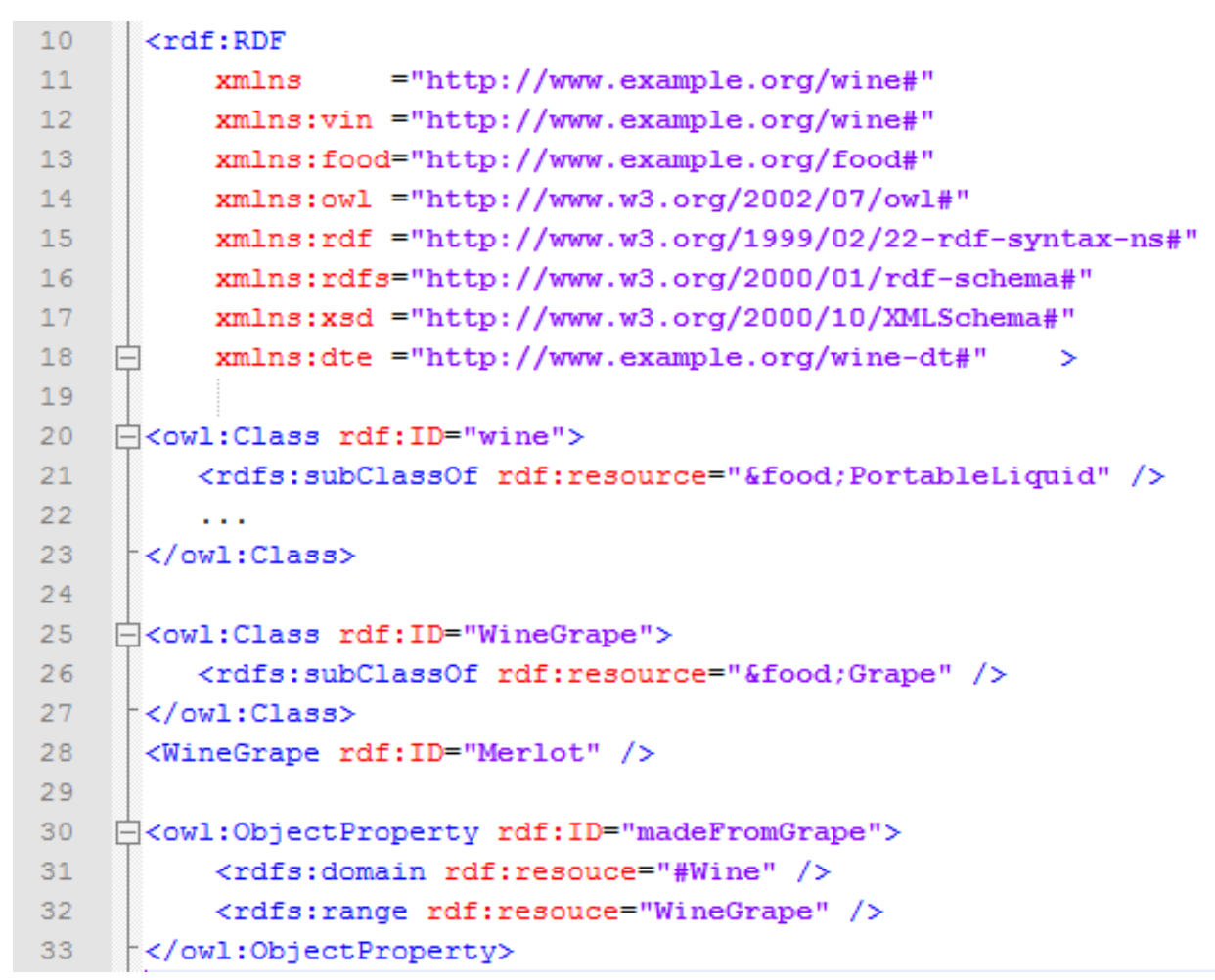

Figura 3.6: Exemplos de Cabeçalho, Classe, Sub-Classe, Instância de Classe ou Indivíduo e Propriedade em OWL (Martimiano, 2006)

namespace xml da ontologia "food" ("comida") e a quarta linha (14) associa a ontologia owl, que permite a utilização dos elementos prefixados com owl: (Classes, Propriedades citadas anteriormente), esse namespace é convencionalmente incluído e utilizado para introduzir o vocabulário OWL. Os demais namespaces (linhas 15, 16 e 17) são associados para indicar elementos de prefixo rdf, rdfs e xsd que definem vocabulários das linguagens RDF, RDF-Schema e XML-Schema respectivamente.

As propriedades especiais citadas anteriormente são exemplificadas pelas Figuras 3.7, 3.8, 3.9 e 3.10. A Figura 3.7 apresenta um exemplo de uma propriedade Transitiva, cujo $I D$ tem o valor de “locatedIn" ( "localizado em”). No exemplo há três regiões (geográficas) que são: a cidade de São Carlos, o Estado de São Paulo e o país Brasil. O Estado de São Paulo ("SaoPauloEstado”) está localizado no Brasil e esses dois indivíduos estão relacionados pela propriedade transitiva "locatedIn”, essa mesma propriedade relaciona os indivíduos SaoCarlos e SaoPauloEstado, sinalizando que São Carlos está localizada no Estado de São Paulo. Assim, por meio dessa propriedade transitiva é possível relacionar São Carlos com Brasil implicando que São Carlos está localizada no Brasil, uma vez que São Carlos está localizada no Estado de São Paulo e este está localizado no Brasil.

A Figura 3.8 possui um exemplo de propriedade simétrica, cujo ID é “adjacentRegion” ( "região adjacente"). Essa propriedade define a simetria entre duas instâncias que no exemplo é a Região de São Carlos ("SaoCarlosRegion") e a Região de Araraquara ("AraraquaraRegion”), a propriedade “adjacentRegion” por ser simétrica, torna os dois indivíduos adjacentes. Outro exemplo clássico 


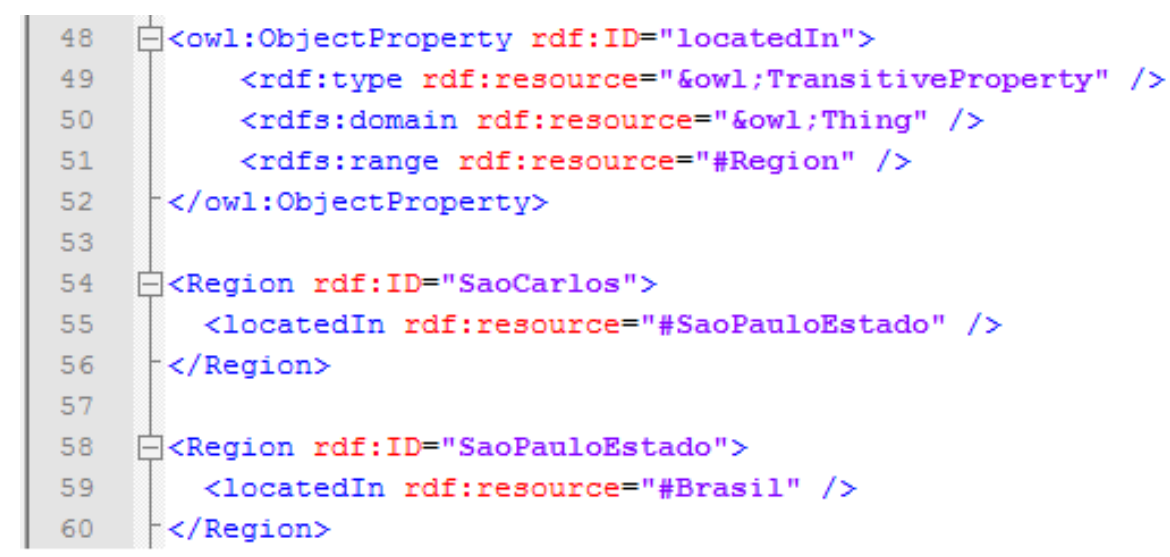

Figura 3.7: Exemplo de propriedade Transitiva

de propriedade simétrica seria uma propriedade de ID igual a "marriedto" (casado com) relacionando dois indivíduos que são casados.

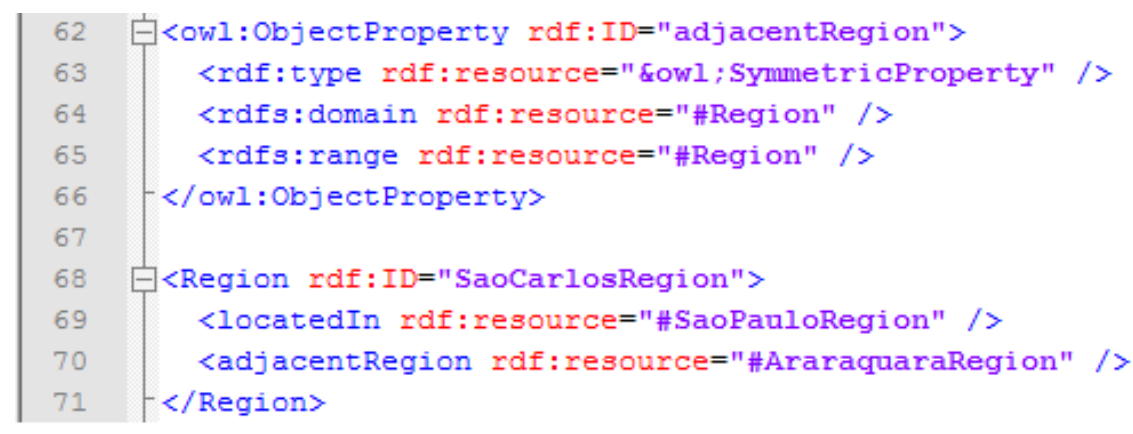

Figura 3.8: Exemplo de propriedade Simétrica

A Figura 3.9 mostra um exemplo de propriedade funcional de ID igual a "hasMayor" ("tem prefeito"), que relaciona um domínio "City" ( "cidade”) com um "Mayor" ("prefeito") e dessa forma esta propriedade implica que uma cidade pode ter apenas um prefeito.

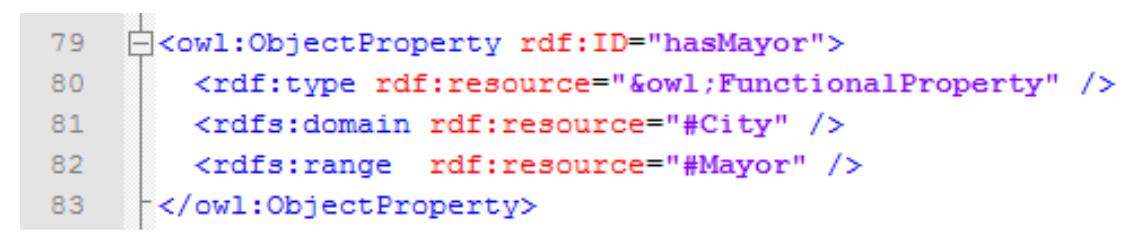

Figura 3.9: Exemplo de propriedade Funcional

A propriedade "biologicalMotherOf” ( "mãe biológica de") na Figura 3.10 é um exemplo de uma propriedade funcional inversa. Essa propriedade relaciona um domínio "Woman” ( "mulher”) com um "Human" ("humano"). De uma perspectiva inversa nota-se que o ser humano deve ter apenas um elemento dentro do domínio "mulher" o qual seria sua mãe biológica (W3C, 2004a).

Todas essas propriedades e outras características sobre a OWL demonstram o quão eficaz essa linguagem pode ser para gerar semântica e para apoiar a representação de ontologias. O sucesso da 


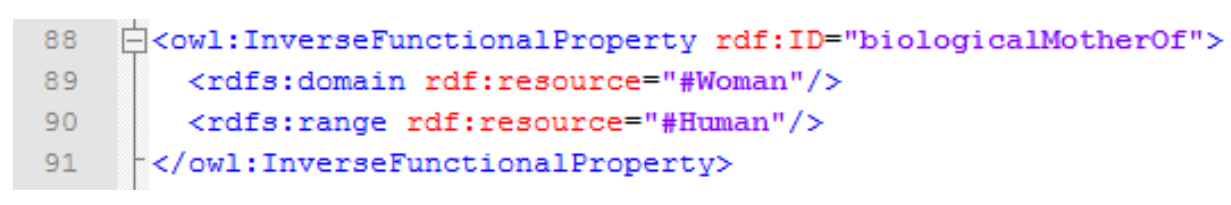

Figura 3.10: Exemplo de propriedade Funcional

linguagem OWL fez com que ela se tornasse o padrão utilizado pela comunidade de Web Semântica, especialmente para descrever ontologias (Isotani et al., 2009). Por esse motivo essa linguagem foi a escolhida para o desenvolvimento da ontologia proposta neste trabalho.

\subsection{A OWL-S - Semantic Markup for Web Services}

A partir da criação da OWL, várias ontologias foram desenvolvidas. Um exemplo é a OWLS (Martin et al., 2004) que propõe uma semântica para Web Services. A OWL-S é baseada em classes e possui a classe "serviço" sendo o ponto de referência para a declaração de um Web Service. Na Figura 3.11, é possível observar o topo do nível de uma ontologia de serviço, em que são observadas a classe "Serviço", a classe "ServiçoFundamento", a classe "ServiçoPerfil” e a classe "ServiçoModelo" (Traduzido de (Martin et al., 2004)).

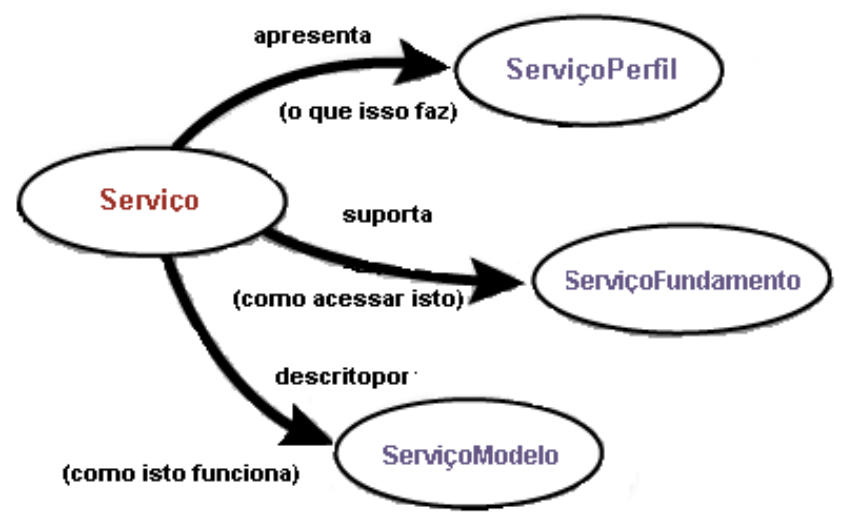

Figura 3.11: Topo do nível de uma ontologia de serviço. Traduzido de (Martin et al., 2004).

A classe "Serviço" representa o serviço Web em si e está relacionada às classes "ServiçoPerfil", "ServiçoFundamento" e "ServiçoModelo" por intermédio das propriedades "apresenta", "suporta" e "descritopor", respectivamente. Essas três classes estão envolvidas com as seguintes funções (Martin et al., 2004):

- ServiçoPerfil: Classe que possui propriedades com informações sobre o serviço, como nome, descrição, categoria, classificação e parâmetros.

- ServiçoModelo: Classe que descreve como o cliente pode consumir o serviço e conhecer o passo a passo do processo até obter os resultados. Informa se é um serviço simples ou composto. 
- ServiçoFundamento: Classe que especifica detalhes de como o serviço pode ser acessado. Geralmente especifica o protocolo de comunicação, o formato das mensagens, as portas de comunicação, etc.

A OWL-S é um exemplo de ontologia, reconhecido pela $\mathrm{W} 3 \mathrm{C}$, para serviços web como um todo. Contudo, outros exemplos de ontologias podem ser desenvolvidos com objetivos mais específicos.

\subsection{Ontologia, Web Semântica e Web Services}

As ontologias têm sido utilizadas em várias áreas na ciência da computação, geralmente naquelas que necessitam de elaboração de conhecimento, semântica e interoperabilidade, como por exemplo, em sistemas inteligentes e de tomada de decisão. Cada área cria e utiliza ontologias da melhor forma que atenda às suas necessidades. Uma ontologia aborda um domínio de conhecimento, os quais geralmente variam de uma área para outra.

Em Sistemas Distribuídos a ontologia é empregada em aplicações que necessitam compartilhar conhecimento e obter semântica. Segundo Zhou et. al. (2005), a semântica aplicada à Web é uma solução para os processos de descoberta de serviços de forma automática; para isso, é necessário que os dados não sejam apenas "lidos" por máquinas, mas também "compreendidos"(Zhou et al., 2005). Como os Web Services são implementações da arquitetura SOA, podem ser largamente utilizados por aplicações como Grades Computacionais (Grids), Intercâmbio Eletrônico, Serviços de Monitoramento, Computação nas Nuvens, etc.

Contudo, a expansão da utilização dos Web Services proporciona preocupação com a qualidade de serviço. Prover QoS em Web Services não é uma tarefa trivial (Lee e Shin, 2008). Clientes, provedores, registros de serviços (UDDIs) e outros elementos que compõem uma arquitetura de Web Service geralmente estão distribuídos em regiões geográficas diferentes e estão sujeitos a sofrer interferências. Um exemplo pode ser quando um provedor de serviço ficar indisponível e nenhuma informação é passada ao UDDI. Nesse caso, esse provedor e sua lista de serviços não ficarão disponíveis para o cliente, que ao tentar acessá-los se deparará com um erro. Outro exemplo é quando o cliente necessita da garantia de que um serviço disponha de uma característica de QoS, e identificar qual serviço ou provedor fornece essa característica se torna um desafio.

Uma solução para o primeiro problema é um sistema de monitoramento, cuja função seria monitorar os provedores de serviços e, quando estivessem indisponíveis, a lista de serviços relacionada a tal provedor seria excluída. Outra solução, ainda para o primeiro problema é a solução proposta na WSARCH (Estrella, 2010), que monitora os provedores de serviços e envia as requisições dos usuários apenas para os servidores que garantam o requisito de QoS desejado. Para o segundo problema uma solução mais elaborada deve ser empregada. Não seria vantajoso consumir o serviço de tempos em tempos para avaliar a qualidade de serviço. Uma solução é "obrigar" o provedor de serviço a se monitorar e garantir a QoS exigida pelos clientes. Para isso, há acordos 
do tipo WSLA entre empresas e clientes. Sendo assim, pode ocorrer então o cenário no qual o cliente deseja um serviço com certos parâmetros de QoS e os provedores garantem que os serviços terão tais características. A questão é descobrir quais são os serviços que atendem às características exigidas. Além disso, é preciso classificar uma enorme variedade de serviços com diversos tipos de parâmetros e métricas de QoS diferentes. Soma-se a isso a criação de uma semântica que decida quais serviços se enquadram nas necessidades do usuário.

Para tentar apresentar soluções a tais problemas, várias pesquisas têm sido desenvolvidas visando a criação e utilização de ontologias (Papaioannou et al., 2006), (Tran, 2008), (Maximilien e Singh, 2004), (Fakhfakh et al., 2008) e (Aklouf e Rezig, 2009). Definir claramente as tarefas que devem ser realizadas é um passo importante para o desenvolvimento de ontologias, uma vez que não existe um consenso sobre qual metodologia deve ser seguida. Normalmente desenvolvedores acabam criando suas próprias ontologias (Martimiano, 2006).

Para exemplificar, considera-se um Web Service que tenha duas implementações disponíveis que realizam a fatoração de um número. O provedor classificou essas duas implementações de serviços em dois domínios diferentes, uma com um serviço em um servidor dedicado e tolerante a falhas, com replicação e segurança garantida (domínio com provisão de QoS), e o outro em um servidor compartilhado vulnerável a vários problemas (domínio sem provisão de QoS). Depois de classificado, o provedor resolveu cobrar pelo serviço no servidor dedicado. Quando o cliente requisitar a lista de serviços disponíveis no UDDI, serão retornados dois serviços. Sem informações suficientes ou sem a utilização de ontologias para criar uma semântica, o cliente não poderia saber qual serviço possui mais garantias de QoS e qual tem o menor custo.

Neste exemplo, surge uma solução mais simples, que seria avisar o cliente de que o serviço localizado no IP ou DNS pré-definido possui um preço superior, pois tem garantias de qualidade de serviço. É uma solução, mas não é interessante, pois na prática servidores dedicados hospedam mais de um serviço e cada um pode ter custos diferentes que envolvam tecnologias e recursos diferentes. Uma melhor solução seria empregar ontologias para classificar os serviços e prover um contexto semântico para que os clientes possam acessar os serviços de acordo com as suas necessidades de uma forma mais dinâmica e automática.

\subsection{Considerações Finais}

Este capítulo inicialmente abordou o conceito de Ontologia, posteriormente o relacionou com Web Semântica e por fim associou os dois assuntos com Web Services. O conceito de ontologia é aplicável na informática quando relacionado ao mapeamento de domínios. A Web Semântica foi apresentada e exemplificada com tecnologias utilizadas de forma sucinta, uma vez que esse assunto abrange uma enorme quantidade de conceitos e existem diversas fontes disponíveis. Além disso, a Web Semântica continua a evoluir e um grande volume de dados tornou-se disponível nos últimos anos (Oren et al., 2009). Foi dado um enfoque para a linguagem OWL, a qual foi 
utilizada neste projeto e também para demonstrar a sua capacidade de utilização e criação de semântica. O capítulo também apresentou uma argumentação sobre a utilização de Web Semântica com ontologia para um enfoque baseado na qualidade de serviços em Web Services. 



\section{CAPÍTULO \\ 4 \\ Ontologia do UDOnt-Q}

\subsection{Considerações Iniciais}

Este capítulo descreve a ontologia que foi desenvolvida especialmente para o módulo UDOnt-Q (Universal Discovery with Ontology and $Q o S$ ). O módulo e os algoritmos de busca serão explicados no próximo capítulo. A ontologia é um elemento importante neste projeto de mestrado, pois é nela que ficam armazenadas as informações de qualidade de serviços que são acessadas pelos algoritmos. Esses por sua vez, têm a função de encontrar os melhores serviços para os clientes. A Figura 4.1 exibe um modelo abstrato da relação entre o cliente, módulo, algoritmos e a ontologia.

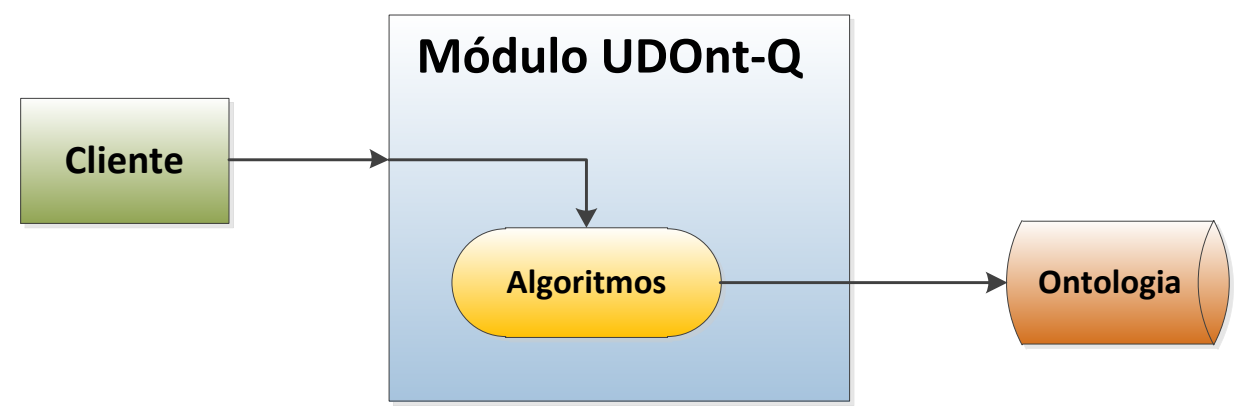

Figura 4.1: Modelo abstrato da relação entre o cliente, módulo, algoritmos e a ontologia.

$\mathrm{Na}$ seção 4.2 são apresentados os processos de desenvolvimento da ontologia, conceitos e ferramentas utilizados. Na seção 4.3 são citados os elementos (classes, subclasses, propriedades) 
que compõem a ontologia. Nessa mesma seção é explicada a organização dos elementos a fim de representar uma arquitetura para Web Services com qualidade de serviço. A seção 4.4 reserva espaço para as considerações finais a respeito deste capítulo.

\subsection{Desenvolvimento da Ontologia do UDOnt-Q}

O desenvolvimento da ontologia seguiu os procedimentos propostos em duas metodologias: Methontology (Lopez et al., 1997) e Metodologia de Noy e McGuinness (Noy e McGuinness, 2001).

\subsubsection{Methontology}

A escolha pela Methontology foi influenciada pelo fato desta ser uma metodologia baseada no padrão IEEE 1074-1995 para desenvolvimento de software (Martimiano, 2006). Essa metodologia consiste em um método estruturado para construir ontologias a partir do zero. Ela leva em consideração o ciclo de vida das ontologias ${ }^{4}$, propondo um protótipo evolutivo composto pelas seguintes fases (Lopez et al., 1997) (Lopez et al., 1999):

1. Aquisição do Conhecimento (Knowledge Acquisition): Esta atividade é independente no processo de desenvolvimento da ontologia, porém coincide com outras atividades. A maior parte da aquisição de conhecimento é feita simultaneamente com a fase de especificação de requisitos. Os especialistas (experts), livros, figuras, tabelas e até mesmo outras ontologias são fontes de conhecimento que podem ser consultados. Podem-se utilizar algumas técnicas durante este processo, como por exemplo: troca de ideias (brainstorming), entrevistas, análises formais e informais de textos, etc.

2. Especificação (Specification): O principal objetivo desta fase é a criação de um documento de especificações estrito em linguagem natural utilizado para caracterizar a ontologia como: Informal, Semi-Formal ou Formal. Além da formalidade, o propósito e escopo (domínio) de cada termo da ontologia devem ser incluídos.

3. Conceituação (Conceptualization): Nesta atividade, o domínio do conhecimento é estruturado em um modelo conceitual que descreve o problema e a sua solução em termos do vocabulário de domínio identificado na atividade de especificação da ontologia. Portanto, facilitando o usuário final a identificar se a ontologia é útil ou não sem precisar inspecionar o código fonte. Adicionalmente, é possível verificar o escopo e a completude de várias ontologias, além da capacidade de reuso e compartilhamento.

\footnotetext{
${ }^{4}$ Segundo Lopez et al. (1997), o ciclo de vida das ontologias é composto por: Especificação, Conceituação, Formalização, Integração, Implementação e Manutenção.
} 
4. Integração (Integration): A atividade de Integração tem o objetivo de acelerar o processo de construção da ontologia considerando o reuso de definições já construídas em outras ontologias ao invés de iniciar do zero. O resultado dessa atividade é um documento de integração que se resume a informações sobre a meta-ontologia que será utilizada e sobre os termos os cujas definições estão sendo utilizadas.

5. Implementação (Implementation): Para a atividade de implementação de ontologias é requerido o uso de ambiente de desenvolvimento que dê suporte à meta-ontologia e às ontologias selecionadas na fase de integração. O ambiente deve realizar análises (sintáticas e léxicas), possuir um editor (para adicionar, remover ou modificar definições), um navegador (browser) para inspecionar as ontologias e suas definições, um pesquisador (searcher) para procurar por definições mais apropriadas e um validador para detectar inconsistências, incompletude e redundância de conhecimento.

6. Avaliação (Evaluation): A avaliação é a realização de um julgamento técnico das ontologias. Ela agrupa dois termos: Verificação (Verification) e Validação (Validation). A verificação se refere ao processo técnico que garante a exatidão de uma ontologia. A validação garante que as ontologias, o ambiente de software e a documentação correspondam ao sistema suposto.

7. Documentação (Documentation): A atividade de documentação é feita durante todo o processo de desenvolvimento da ontologia. A cada fase é gerado um documento, por exemplo, após a fase de aquisição do conhecimento, um documento "aquisição do conhecimento" é gerado. Esse processo é repetido até a fase de Avaliação.

O desenvolvimento da ontologia procurou seguir o ciclo de vida proposto anteriormente e a execução de cada fase foi realizada da seguinte forma:

1. Aquisição do Conhecimento: O conhecimento sobre o domínio referente à arquitetura de Web Services com qualidade de serviço foi obtido da leitura e análise de livros, artigos e até mesmo da ontologia da OWL-S. Outra fonte de conhecimento utilizada foi através de entrevistas e troca de ideias com especialistas da área de sistemas distribuídos (professores, alunos de doutorado e mestrado). A conclusão dessa fase foi que uma ontologia menos complexa que da OWL-S poderia ser desenvolvida levando em consideração a parte de acordos de níveis de serviços (SLA) entre os clientes e provedores de serviço. Os detalhes da aquisição de conhecimento foram obtidos paralelamente à fase de Especificação.

2. Especificação: Na fase de especificação foram descritos para cada termo (elemento) da ontologia a sua formalização, escopo (domínio) e propósito. Esta fase foi útil para definir os elementos que compõem a ontologia, detalhando informações e especificando a função (propósito) de cada um deles. O resultado dessa fase foi a criação de um documento de especificações que se encontra ao final deste trabalho (Anexo 1). 
3. Conceituação: Nesta fase foram criados diversos documentos buscando-se modelar o conceito da ontologia a fim de representá-la facilitando o seu entendimento para os usuários. Alguns desses documentos recomendados por Lopez et al.(1999) são: Glossário de Termos (Glossary of Terms), Árvore de Classificação de Conceitos (Concept-Classification Tree), Diagramas de Relações Binárias (Binary-Relations Diagrams), Dicionário de Conceitos (Concept Dictionary), Tabelas de Relações Binárias (Binary-Relations Tables), Tabela de Instâncias (Instance Table) e Tabela de Atributos das Instâncias (Instance-attribute Table). Todos esses documentos fornecem informações relevantes antes do processo de implementação da ontologia e estão disponíveis no final deste trabalho (Anexo 2).

4. Integração: Esta etapa não foi considerada no desenvolvimento da ontologia do UDOnt-Q, pois, ela foi projetada para ser simples e servir apenas como base de conhecimento para o módulo UDOnt-Q. Portanto, ela foi criada a partir do zero e não houve integração com outras ontologias.

5. Implementação: A recomendação de Lopez et al. (1999) era a utilização do ODE (Ontology Design Environment) para apoiar o projetista de ontologias no desenvolvimento da ontologia. Porém, uma ferramenta mais atual e amplamente conhecida para o desenvolvimento de ontologias é o Protégé ${ }^{5}$. A escolha pelo Protégé é justificada pelo fato dele trabalhar com a linguagem OWL (apresentada no capítulo anterior). Mais informações sobre o Protégé e sobre a implementação da ontologia estão na subseção 4.2.3.

6. Avaliação: A ontologia foi submetida a avaliações por meio da análise técnica de seus desenvolvedores e também por meio de verificações e validações feitas pelo Protégé em conjunto com máquinas de inferências (racionalizadores - reasoners). Esta fase foi útil para correções e eliminação de termos (elementos e classes) desnecessários. Por exemplo, inicialmente na ontologia havia classes de tipos (TypeClass) que definiam um tipo para cada serviço, cliente e qualidade de serviço. Essas classes de tipo foram substituídas por subclasses que possuem características que determinam um "tipo" específico de cliente, serviço e QoS.

7. Documentação: Esta fase foi cumprida e resultou em diversos documentos e informações que estão apresentados neste capítulo e em alguns anexos deste trabalho.

\subsubsection{Metodologia de Noy e McGuinness}

A Metodologia de Noy e McGuinness (2001) também teve influência no desenvolvimento da ontologia deste projeto, principalmente na parte de implementação. O documento "Ontology Development 101: A Guide to Creating Your First Ontology" que expõe uma metodologia para a criação de ontologias é também um guia prático de desenvolvimento. Portanto, além de todo o conteúdo teórico, esse documento também oferece exemplos de desenvolvimento prático, utilizando

\footnotetext{
${ }^{5}$ http://protege.stanford.edu/
} 
a ferramenta Protégé. Essa mesma ferramenta, em uma versão mais atualizada, foi emprega no desenvolvimento da ontologia do UDOnt-Q.

Os autores dessa metodologia afirmam que: “não há nenhum modo 'correto' ou metodologia para o desenvolvimento de ontologias" (Noy e McGuinness, 2001). Contudo, eles propõem um possível processo de desenvolvimento baseado em sete etapas, como descritas a seguir (Noy e McGuinness, 2001):

1. Determinar o domínio e escopo da ontologia (Determine the domain and scope of the ontology): Nesta etapa os autores propõem que os projetistas de ontologias respondam a algumas perguntas básicas com o objetivo de determinar o domínio e escopo da ontologia.

2. Considerar o reuso de ontologias existentes (Consider reusing existing ontologies): Os autores argumentam que muitas ontologias já estão disponíveis em formato eletrônico e encorajam que sejam reutilizadas.

3. Enumerar os termos importantes na ontologia (Enumerate important terms in the ontology): Documentar uma lista de todos os termos que os projetistas de ontologias desejam pode ser útil para criar afirmações sobre eles ou explicá-los para um usuário. É preciso acrescentar informações sobre os termos e quais são as suas propriedades.

4. Definir as classes e a hierarquia de classe (Define the classes and the class hierarchy): Os conceitos da ontologia podem ser classificados ou agrupados em classes e subclasses que podem definir uma taxonomia proporcionando uma hierarquia de classe. Os autores citam três propostas para o desenvolvimento da hierarquia de classe (Uschold e Gruninger, 1996):

a. Top-Down: O desenvolvimento inicia pela definição dos conceitos mais gerais no domínio da ontologia e seguem até os conceitos mais específicos.

b. Bottom-Up: É o contrário do Top-Down: o desenvolvimento inicia pela definição dos conceitos mais específicos no domínio da ontologia e seguem até os conceitos mais gerais.

c. Combination: O desenvolvimento segue uma combinação das duas propostas anteriores: Top-Down e Bottom-Up.

5. Definir as propriedades (slots) das classes - (Define the properties of classes-slots): As propriedades das classes (slots) devem ser definidas com o objetivo de prover informação suficiente para responder a questões sobre o domínio da ontologia.

6. Definir as facetas para as propriedades (slots) (Define the facets of the slots): As propriedades (slots) podem ter facetas diferentes para descrever características como: tipo de valor (Value Type) ou tipo de dado, por exemplo: String, Number, Boolean, etc., cardinalidade (Cardinality) e domínio e variação (Domain and Range) que relaciona a propriedade com classes e tipos de instâncias respectivamente. 
7. Criar Instâncias (Create instances): A última etapa proposta pelos autores é a criação de instâncias de classes, também chamados de indivíduos da ontologia. Um indivíduo de uma classe possui todas as atribuições (propriedades) de sua classe, ele é utilizado na representação de seres do mundo real (dentro do escopo/domínio definido).

É possível notar algumas semelhanças entre as duas metodologias que foram estudas para o desenvolvimento da ontologia deste trabalho. A etapa 1 da metodologia de Noy e McGuinness sintetiza as fases 1 e 2 da Methontology. A etapa 2 é correspondente à fase 4 da Methontology. As etapas restantes podem se comparadas às fases 3 e 5 da Methontology. Apesar da Metodologia de Noy e McGuinness não ter fases específicas para as etapas 6 e 7 da Methontodoly, no decorrer do documento atividades semelhantes são comentadas. Por outro lado, o documento é rico em sugestões e exemplos práticos, guiando o desenvolvedor na criação de sua primeira ontologia.

\subsubsection{Ferramentas para o desenvolvimento da Ontologia}

Para o desenvolvimento da ontologia foram utilizadas três ferramentas: o Protégé, o racionalizador Pellet ${ }^{6}$ e uma aplicação denominada UDOnt-Q WorkLoad. Esta última foi desenvolvida em Java com recursos da Web Semântica para agilizar o processo de criação de indivíduos na ontologia.

\subsubsection{Protégé}

Para a criação da ontologia com a linguagem OWL foi utilizada a ferramenta Protégé criada pelo Centro de Pesquisas para Informática Biomédica da Universidade de Stanford (Gennari et al., 2002). A ontologia desenvolvida foi salva em um arquivo, cujo nome é "UDOnt-Q_Ontology.owl" e disponibilizada por meio de uma URI: http://localhost:8080/ontologies/UDOnt-Q_Ontology.owl no servidor Web Apache.

Inicialmente uma ontologia em OWL criada no Protégé possui uma classe padrão (default) denominada "Thing" (owl:Thing), que constitui a classe raiz dentro de uma hierarquia de classes na ontologia; todas outras classes criadas ficam abaixo da classe "Thing". A Figura 4.2 mostra a hierarquia de classes da ontologia do UDOnt-Q mapeada pelo plugin OWLViz?

O primeiro passo da implementação da ontologia foi a criação das classes. Adotou-se a proposta Top-Down, para o desenvolvimento da hierarquia de classes. Além disso, foram criadas propriedades (slots) para o relacionamento entre as classes (Object Properties) e propriedades entre classes e dados (Data Properties). O Protégé disponibiliza abas para a criação de tais propriedades, nas quais podem ser especificados os domínios e variações (domain and ranges).

\footnotetext{
${ }^{6} \mathrm{http}: / /$ clarkparsia.com/pellet

${ }^{7}$ OWLViz é um OWL plugin para Protégé, ele permite a visualização e a navegação incremental da hieraquia de classes em OWL, permitindo a comparação da hierarquia de classe afirmada (asserted) e da hierarquia de classe inferida (inferred) (http://www.co-ode.org/downloads/owlviz/).
} 


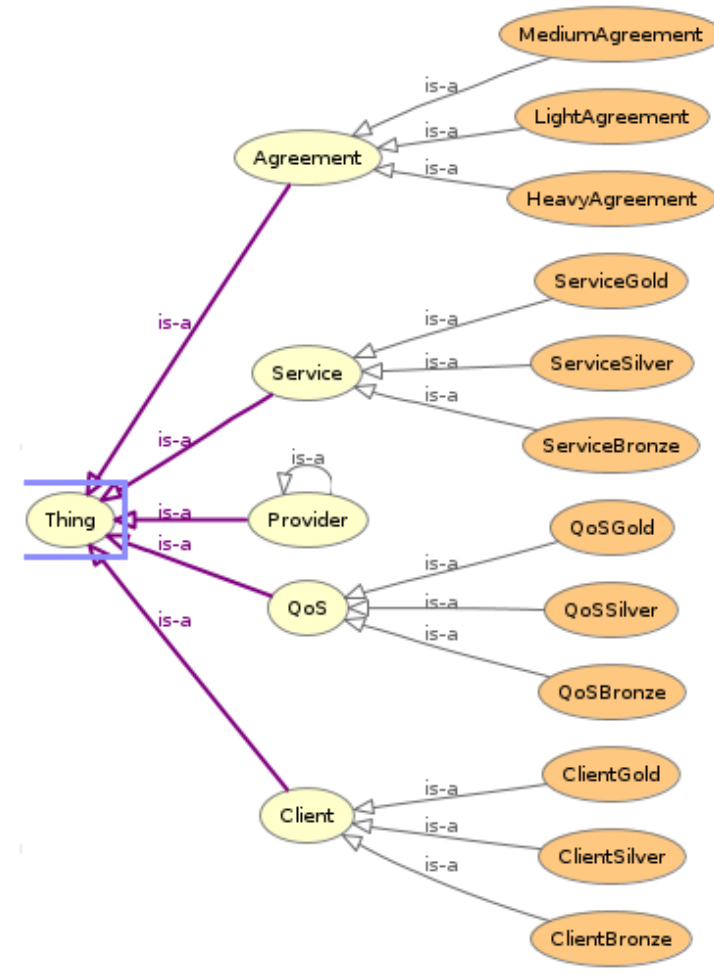

Figura 4.2: Mapeamento da Hierarquia de Classes do UDOnt-Q. (Nakamura et al., 2011a)

A Figura 4.3 exibe uma parte da aba para criação de propriedades entre classes (Object Properties).

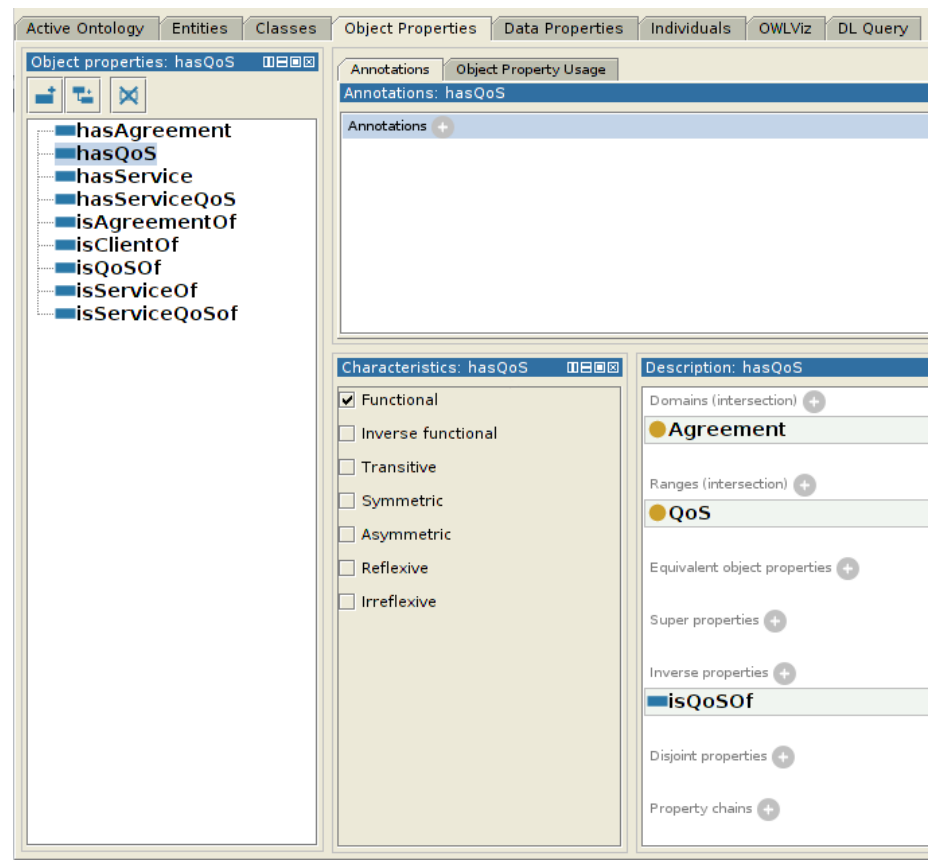

Figura 4.3: Parte da aba de propriedades entre classes (Object Properties).

O Protégé também possui uma aba específica para a criação de indivíduos, onde, o desenvolvedor pode criar, excluir e manipular dados de indivíduos. Pode-se definir um tipo para o indivíduo, 
por exemplo, indicando que ele representa uma instância de uma determinada classe. As classes, propriedades e indivíduos criados na ontologia são explicados em mais detalhes na seção 4.3.

Além de facilitar e agilizar a criação de ontologias, o Protégé também conta com outro ponto interessante que é a existência de máquinas de inferências (racionalizador - reasoners). Elas oferecem diversos serviços, que permitem realizar diversas consultas e validações na ontologia. A máquina de inferência utilizada neste trabalho foi a Pellet e mais detalhes sobre ela são apresentados na próxima subseção.

\subsubsection{Pellet}

Pellet é escrita em Java e possui seu código aberto sob uma licença de utilização liberal ${ }^{8}$. Ele avalia ontologias desenvolvidas com a linguagem OWL DL (OWL Description Logics), verificando se essas ontologias estão de acordo com os serviços de inferência definidos pela lógica de descrição (DL), denominados (Sirin et al., 2007):

- Consistência: Assegura que uma ontologia não contém qualquer fato contraditório.

- Conceito de satisfação: Verifica se é possível uma classe possuir instâncias. Se a classe não satisfaz, então a definição de uma instância da classe causará a inconsistência de toda a ontologia.

- Classificação: Processa as relações entre as classes e subclasses para determinar a hierarquia.

- Realização: Considera as classes mais específicas que um indivíduo pertence, encontrando assim as instâncias (tipos diretos - direct types) de um indivíduo.

Dessa forma, a máquina de inferência Pellet oferece recursos úteis para avaliar ontologias criadas na linguagem OWL-DL. Por este motivo ela foi adicionada ao Protégé e utilizada no processo de desenvolvimento da ontologia e na criação do módulo UDOnt-Q.

\subsubsection{UDOnt-Q WorkLoad}

Para representar um ambiente real pode ser necessária a criação de diversos indivíduos de diferentes tipos (classes). A criação manual de uma grande quantidade de indivíduos por meio da ferramenta Protégé é uma tarefa trabalhosa, principalmente se eles possuírem diversas propriedades de objetos e dados.

A ferramenta UDOnt-Q WorkLoad foi desenvolvida com o objetivo de acelerar o processo de criação de indivíduos. Ela foi desenvolvida com na linguagem Java e faz uso de um componente

\footnotetext{
${ }^{8}$ Pellet está sob a licença AGPL v. 3 (http://www.fsf.org/licensing/licenses/agpl-3.0.html)
} 
denominado (CIS - Common Information Shared) pertencente ao módulo que será apresentado no capítulo 5.

O funcionamento dessa ferramenta é apresentado no final da próxima seção, pois para o melhor entendimento é interessante ter conhecimento dos elementos e propriedades da ontologia.

\subsection{Elementos da Ontologia do UDOnt-Q}

A ontologia destinada ao módulo UDOnt-Q foi criada com o intuito de representar os principais elementos participantes do domínio de uma arquitetura de Web Services com QoS. Alguns desses elementos merecem destaque (Nakamura et al., 2011a):

- Clients: Os clientes são os elementos que requisitam serviços com qualidade, para isso eles assinam acordos com os provedores de serviços.

- Providers: Os provedores de serviços devem prover os serviços web com a qualidade adequada, caso contrário podem estar sujeitos a penalidades e/ou multas.

- Services: Representam os Web Services que são providos pelos provedores, eles também são consumidos pelos clientes. Os Web Services devem ser analisados e suas características funcionais e principalmente as não funcionais (valores e atributos de QoS) devem ser especificados.

- Agreements: São os acordos estabelecidos entre os clientes e os provedores. O acordo de um cliente indica qual é a QoS desejada por ele.

- QoS: É a qualidade de serviço pertencente a um determinado serviço ou aquela contratada em um determinado acordo, ela contém níveis e atributos de qualidade.

Cada um dos elementos é representado por uma classe na ontologia do UDOnt-Q que pode ter subclasses, constituindo assim uma hierarquia de classes. Além disso, elas podem estar relacionadas entre si ou relacionadas com dados por meio de propriedades (Object/Data Properties). Essas propriedades podem ter atributos ou características que expressam informações sobre o relacionamento e, dessa forma, a máquina poderá ser capaz de "entender" o significado semântico do relacionamento criado por uma propriedade. A linguagem OWL utilizada no desenvolvimento da ontologia possui propriedades que permitem expressar tais informações.

As propriedades que relacionam classes (Object Properties) na ontologia são:

- hasQoS: é uma propriedade funcional que relaciona a classe Agreement com a classe QoS. Ela implica que um Acordo deve estar relacionado com apenas uma QoS ("tem QoS"). A propriedade inversa a esta é a isQoSOf. 
- hasAgreement: é uma propriedade funcional que relaciona a classe Client com a classe Agreement. Ela indica que um Cliente deve estar relacionado com apenas um acordo ("tem acordo"). A propriedade inversa a esta é a isAgreementOf.

- hasService: é uma propriedade que pode relacionar a classe Provider com a classe Service ("tem Serviço"). Esta classe não é funcional, podem existir provedores com mais de um serviço e também provedores que não disponham de nenhum serviço. A propriedade inversa a esta é a isServiceOf.

- hasServiceQoS: é uma propriedade funcional que relaciona a classe Service com a classe QoS (“tem Serviço QoS”). Ela exige que um Serviço esteja relacionado com uma QoS. A propriedade inversa a esta é a isServiceQoSof.

Além das propriedades de objetos, também foram criadas diversas propriedades que relacionam dados (Data Properties). O objetivo principal dessas propriedades é armazenar informações sobre as instâncias de classes (indivíduos) na ontologia. Portanto, a maior parte do conhecimento da ontologia está localizada em propriedades de dados instanciadas nos indivíduos. Alguns exemplos de propriedades de dados são:

- hasServiceName: propriedade com o tipo de dado "String" que é utilizada para armazenar o nome de um individuo da classe Service.

- hasClientAddress: propriedade com o tipo de dado "String" que é utilizada para armazenar o endereço (IP) de um indivíduo da classe Client.

- hasServiceAddress: propriedade com o tipo de dado "String" que é utilizada para armazenar o endereço (IP) de um indivíduo da classe Service.

- hasResponse_TimeContentValue: propriedade com o tipo de dado "Double" que é utilizada para armazenar o valor (nível) de tempo de resposta (em milissegundos) de um indivíduo da classe QoS. Dessa forma, pode-se informar ou recuperar, o nível do atributo "tempo de resposta" de uma determinada qualidade de serviço. Essa qualidade pode ser tanto de um acordo estabelecido entre os clientes e os provedores, como a qualidade de um serviço que foi previamente analisado pelo provedor.

Para cada atributo de QoS há uma propriedade de dado correspondente na ontologia e diversas outras que agregam informações sobre os seus elementos (Clientes, Serviços, Provedores, etc.).

Das cinco classes, quatro delas possuem três subclasses, conforme exibe a Figura 4.2 na seção anterior. As classes Client, Service e $Q o S$ possuem três subclasses que correspondem aos clientes, serviços e qualidades de serviço do tipo ouro (Gold), prata (Silver) e bronze (Bronze) respectivamente. O que determina se um serviço pertence a uma determinada subclasse é a sua QoS (relacionado pela propriedade hasService $Q o S$ ), ou seja, se um serviço está relacionado a uma $Q o S G o l d$ 
então ele é inferido como sendo um ServiceGold. Da mesma forma, o cliente está relacionado a um acordo (Agreement) que pode pertencer a uma das três subclasses (HeavyAgreement, MediumAgreement e LightAgreement). Cada acordo está relacionado a uma $Q o S$ que determina a sua subclasse, ou seja, se um acordo está relacionado com a QoSGold então ele é inferido como um HeavyAgreement, pois a QoSGold exige que o acordo seja rigoroso. Para determinar se uma $Q o S$ é Gold, Silver ou Bronze a máquina de inferência deve verificar os valores (níveis) dos atributos de cada $Q o S$ e por meio de comparações com as restrições de "Equivalências de Classes", assim ela determina qual é a subclasse desta $Q o S$. A ideia de utilizar restrições para equivalências de classes foi motivada por exemplos em um tutorial que faz uso da ontologia Pizza ${ }^{9}$. A Figura 4.4 exibe um exemplo das restrições de equivalência da subclasse QoSGold. As restrições (ou condições) para que qualquer outro elemento da ontologia seja um elemento equivalente a classe QoSGold são as seguintes (Nakamura et al., 2011a):

- Ter a classe QoS como classe pai (superclass) ou ser subclasse de QoS.

- Ter o valor da propriedade hasResponse_timeContentValue menor ou igual a 500.00 ( significa que o tempo de resposta deve ser menor ou igual a 500 milissegundos).

- Possuir o valor da propriedade hasCostContentValue maior ou igual a 1.00 (significa ter o custo maior ou igual a 1 unidade monetária).

- Possuir o valor da propriedade hasAvailabilityContentValue maior ou igual a 98.00 (significa ter uma disponibilidade do serviço maior ou igual a $98 \%$ do tempo).

\begin{tabular}{|c|}
\hline Description: QoSGold \\
\hline Equivalent classes + \\
\hline OhasResponse_TimeContent/lulue some float[ $<=500.00]$ \\
\hline has $C_{0}$ stContent"yalue some float[ $\left.>=1.00\right]$ \\
\hline Ohaskvailability Content'/alue some float[>=98.00] \\
\hline Superclasses $\biguplus$ \\
\hline $\mathrm{Q}_{0} \mathrm{~S}$ \\
\hline
\end{tabular}

Figura 4.4: Restrições de Equivalência da classe QoSGold.

Da mesma forma existem restrições de equivalências de classe para as subclasses QoSSilver e QoSBronze. Porém os valores dos intervalos em cada propriedade são diferentes. No caso da subclasse QoSSilver os valores dos atributos de QoS devem estar em um intervalo entre (between) dois

\footnotetext{
${ }^{9}$ A ontologia Pizza (http://www.co-ode.org/ontologies/pizza/pizza.owl) é um exemplo utilizado no tutorial pela Universidade de Manchester na Edição 1.2 de Março de 2009 o qual exibe um exemplo da utilização de equivalência de classe por restrições de propriedades de dados. Este exemplo está localizado no Capítulo 5 desse tutorial (Holger et al., 2009).
} 
valores, sendo o limite superior o valor mínimo do atributo da QoSGold e o limite inferior o valor máximo do atributo da QoSBronze. Por exemplo, para equivalência com a subclasse QoSBronze as restrições exigiam que a propriedade hasResponse_timeContentValue tivesse valor maior que 1000 (1 segundo), a propriedade hasAvailabilityContentValue valor menor que 95 (disponibilidade menor que 95\%) e a propriedade hasCostContentValue tivesse um valor menor que 0.50 (custo menor que 0.50 de uma unidade monetária). Para a equivalência com a subclasse QoSSilver as restrições exigem que as propriedades tenham valores limitados. Os valores do limite superior devem estar abaixo dos valores das propriedades da subclasse QoSGold e os valores do limite inferior devem estar acima dos valores das propriedades da subclasse QoSBronze.

Uma vez consistente a ontologia pode ser utilizada como base de conhecimento para buscas semânticas e a classificação por meio da inferência permite que novos indivíduos possam ser criados para representar o mundo real sem a necessidade de serem previamente rotulados com relação a sua qualidade, pois um serviço pode ser criado na ontologia como sendo um individuo da classe Service (superclass) que possui uma qualidade de serviço genérica (individuo da classe QoS (superclass)). Dessa forma, no momento que a máquina de inferência inferir sobre a ontologia, ela irá analisar as restrições e classificar esse indivíduo como equivalente a uma das três subclasses (realizando assim o processo de classificação).

Para encontrar o serviço adequado, o algoritmo deve primeiramente buscar o indivíduo que representa o cliente dentro da ontologia. Para isso, ele compara o atributo de endereço do cliente na ontologia (hasClientAddress) com o IP recuperado pelo módulo. Após encontrar o cliente na ontologia, deve-se buscar o seu acordo e a partir dele é possível identificar a QoS acordada. Quando inferida, esta QoS será equivalente a uma das três subclasses (QoSGold ou QoSSilver ou QoSBronze), o algoritmo deve busca os serviços que possuam QoS na mesma subclasse da QoS do acordo do cliente.

Dessa forma, a seleção de serviços com qualidade é realizada sem a preocupação de uma análise humana, basta que as QoSs dos serviços sejam cadastradas corretamente e que os limites e intervalos que classificam os acordos sejam coerentes nas restrições de equivalência de classe de cada subclasse de QoS.

Diversos indivíduos podem ser criados na ontologia para representar os provedores, serviços, clientes, acordos, QoS do mundo real. Apesar da criação de indivíduos no Protégé ser simples, em grande quantidade esta atividade torna-se muito trabalhosa, entediante e consequentemente poderá ocasionar erros. Por esse motivo, foi desenvolvida a ferramenta (UDOnt-Q WorkLoad) citada anteriormente na subseção 4.2.3.3.

Nessa ferramenta, os indivíduos, propriedades e suas respectivas informações são criados de forma incremental e alguns valores de propriedades de dados são atribuídos de forma aleatória. Contudo, os valores aleatórios devem estar dentro de um intervalo definido pelo o desenvolvedor. Por exemplo, foram criados indivíduos de serviços: USPService1, USPService2, USPService3, ... , USPService1200; para cada individuo de serviço foram criados indivíduos de QoS: USPService1QoS, USPService2QoS, USPService3QoS, ... , USPService1200QoS, que foram relaciona- 
dos pela propriedade “hasServiceQoS". Cada indivíduo de QoS possui um valor (nível) do atributo Tempo de Resposta que é informado em uma propriedade de dado ("hasResponse_TimeContentValue"). Este valor, gerado aleatoriamente, deve estar dentro de um intervalo definido pelo desenvolvedor. Assim, pode-se inferir e definir quais serão as classes dos serviços e das qualidades de serviço. Da mesma forma são criados os outros elementos e propriedades da ontologia.

No Anexo 3 desta monografia é possível observar como foi feita a criação de alguns elementos da ontologia.

\subsection{Considerações Finais}

Neste quarto capítulo foram apresentadas as duas metodologias utilizadas no desenvolvimento da ontologia proposta para o módulo UDOnt-Q. As ferramentas utilizadas para a implementação da ontologia também foram descritas. Ao final os elementos da ontologia foram explicados em conjunto com o funcionamento semântico que o algoritmo deve seguir para selecionar Web Services com a qualidade de serviço acordada pelos clientes. 



\section{UDOnt-Q - Universal Discovery with Ontology and QoS}

\subsection{Considerações Iniciais}

Este capítulo aborda o desenvolvimento de um módulo denominado UDOnt-Q (Universal Discovery with Ontology and $Q o S$ ) com a função de pesquisar serviços no registro de serviços (UDDI) baseando-se em parâmetros de QoS. Para realizar tal pesquisa, o módulo faz uso de recursos da Web Semântica e da ontologia apresentada no capítulo anterior. Na seção 5.2 são apresentados brevemente a metodologia e as ferramentas utilizadas para o desenvolvimento do UDOnt-Q. Na seção 5.3 são apresentados os componentes e a estrutura do módulo. Na seção 5.4 são apresentadas as considerações finais deste capítulo.

\subsection{Metodologia e Ferramentas}

Em uma arquitetura para Web Services com QoS há diversas informações sobre os provedores, serviços, acordos e clientes que geralmente não estão disponíveis, e quando estão nem sempre estão organizadas de uma forma explícita com uma semântica que permita que agentes computacionais façam inferências e tomem decisões como, por exemplo, qual(is) o(s) melhor(es) serviço(s) que atende $(m)$ a um acordo de um determinado cliente? Uma opção para organizar tais informações da última forma descrita é por meio da criação de ontologias. Portanto, as ontologias podem representar uma base de conhecimento. Contudo, seria muito trabalhoso para o ser humano acessar e 
verificar informações na ontologia manualmente. Esse problema aumenta dependendo do tamanho da ontologia e caso seja necessário realizar inferências de algumas informações, o espaço temporal para isso seria enorme e poderia resultar em erros.

Para solucionar tais problemas, empresas como a HP e grupos de pesquisa em Web Semântica desenvolveram APIs e ferramentas para que as ontologias fossem utilizadas, manipuladas e inferidas por programas de computador. Um exemplo de API para acesso e manipulação de ontologia é o Jena, discutido na subseção 5.2.2. Outra ferramenta, comentada anteriormente na seção 4.2.3, é o Pellet, utilizado para realizar validações, verificações e inferências na ontologia. Porém, para fazer uso dessas ferramentas se fez necessário o desenvolvimento do módulo UDOnt-Q. Pelo fato dos códigos fontes do Jena e Pellet serem implementados na linguagem Java, o módulo também foi desenvolvido nesta mesma linguagem para facilitar a integração entre eles. Além disso, a linguagem Java é amplamente utilizada em trabalhos acadêmicos, pois ela é livre, produtiva, orientada a objetos e multi-plataforma.

\subsubsection{Metodologia Ágil (Agile)}

A metodologia ágil (Agile) de desenvolvimento de software propõe a utilização de processos mais integrados e ágeis entre a equipe de desenvolvimento e o cliente promovendo entregas contínuas (ou incrementais) de software com algum valor agregado. Essa metodologia não se prende tanto às documentações e as informações de maior valor são passadas diretamente pelos clientes. Segundo Ambler (2002), há quatro declarações de valores que definem o manifesto ágil (Ambler, 2002):

- "Indivíduos e interações acima de processos e ferramentas";

- "Software funcional acima de documentação compreensiva";

- "Colaboração do cliente acima da negociação do contrato";

- "Respondendo as mudanças acima de seguir um plano";

O desenvolvimento do módulo UDOnt-Q foi baseado na metodologia ágil e a opção por sua utilização é justificada por proporcionar uma maior interação, facilidade de alterações no decorrer do desenvolvimento e, principalmente, resultados mais rápidos com entregas continuas de partes funcionais que agregam valor ao produto final. Uma parte da documentação mínima produzida neste trabalho de mestrado é apresentada na seção 5.3.

\subsubsection{Ferramentas}

Diversas ferramentas foram empregadas para o desenvolvimento do módulo. Além do ambiente integrado de desenvolvimento (IDE - Integrated Development Environment), que no caso deste 
projeto foi utilizado o Eclipse ${ }^{10}$, outras bibliotecas foram utilizadas como, por exemplo, drivers de conexão para acesso ao banco de dados MySQL ${ }^{11}$.

As principais ferramentas utilizadas pelo o módulo que merecem destaque são:

- Pellet: Máquina de Inferência (racionalizador - reasoner) Pellet foi comentada anteriormente na seção 4.2.3. Acrescenta-se ainda que Pellet na versão 2.2.2 possui um pacote específico para consultas em arquivos OWL-DL (pellet-query-src.jar). Nesse pacote, dentro do namespace (com.clarkparsia.pellet.sparqldl) há classes que permitem realizar, programaticamente, consultas na ontologia com a linguagem SPARQL-DL (SPARQL Query for $O W L-D L$ ) (Sirin e Parsia, 2007). Essa linguagem é uma variação da linguagem de consulta SPARQL (SPARQL Protocol and RDF Query Language) (Prud'hommeaux e Seaborne, 2008). Ambas possuem algumas semelhanças com a linguagem de consulta SQL (Structured Query Language). SPARQL é uma recomendação proposta pela W3C que se baseia no conceito dos padrões de triplas do grafo RDF e sua semântica está centrada na correspondência nas triplas do grafo RDF (Sirin e Parsia, 2007). Contudo, há questões de compatibilidade entre as consultas com SPARQL e bancos de dados OWL-DL. Por esse motivo, a SPARQLDL foi proposta com uma sintaxe abstrata específica para ela, que define uma semântica baseada no modelo teórico da OWL-DL. Além disso, um mapeamento é realizado dessa sintaxe abstrata para a forma de grafo RDF (Sirin e Parsia, 2007) (Malik et al., 2010). O fato de a ontologia criada ser na linguagem OWL e a prévia inclusão dos pacotes do Pellet no módulo justificam a utilização da linguagem de consulta SPARQL-DL em um dos algoritmos de busca do UDOnt-Q.

- Jena: Framework ou API de desenvolvimento de aplicações com Web Semântica, discutido na subseção 5.2.2.

- $\log 4 \mathbf{j}$ : O $\log 4 \mathrm{j}$ é uma ferramenta que ajuda o programador no registro de informações de saída $(\log s)(\log 4 \mathrm{j}, 2101)$. Desenvolvida pela Apache Software Foundation, o $\log 4 \mathrm{j}$ é um pacote de classes em Java que, quando utilizado por um programa, permite que as saídas de informações sejam "impressas" tanto no console como em arquivos. O programador tem a opção de escolher o nível (level) de cada log (por exemplo: ALL, DEBUG, TRACE, INFO, WARM, ERROR, OFF) a fim de rotular o tipo de mensagem de saída. No módulo foram utilizados os níveis DEBUG e ERROR durante os testes iniciais, para verificar valores de parâmetros e eventuais erros respectivamente. Posteriormente, os níveis ALL e OFF foram utilizados durante os experimentos para ora registrar qualquer tipo saída (debug, error, etc.) e ora não registrar as saídas respectivamente.

\footnotetext{
${ }^{10} \mathrm{http}: / / w w w . e c l i p s e . o r g /$

${ }^{11}$ Por exemplo, o driver mysql-connector-java permite que aplicações em Java acessem o banco de dados MySQL. Ele está disponível em http://www.mysql.com/products/connector/
} 
- jUDDI: O jUDDI é uma implementação em Java do UDDI ${ }^{12}$, possui o seu código aberto e pode trabalhar com diversos tipos de banco de dados como, por exemplo o MySQL, Oracle, DB2, entre outros, para armazenar informações de Web Services.

- Ganglia: O Ganglia é um monitor de sistemas escalável e distribuído (Massie et al., 2003), que possui uma comunicação ponto-a-ponto entre os nós mestre e escravos e, por este motivo, ele é utilizado em federações de clusters (Estrella, 2010) (Matos, 2009). Assim, ele foi utilizado neste trabalho para monitorar as máquinas dos provedores de serviço. Os nós escravos monitoram os provedores de serviços e coletam algumas informações de desempenho (CPU e Memória). Tais informações são enviadas ao nó mestre, que as disponibiliza em formato XML, em uma determinada porta previamente configurada. Logo, o módulo abre uma conexão TCP via socket e recupera essas informações, que serão úteis na escolha do melhor provedor de serviço, pois, no caso de dois serviços com QoS semelhantes, o módulo poderá optar por escolher o provedor que estiver menos sobrecarregado naquele determinado instante.

\subsubsection{Jena}

O framework Jena é largamente utilizado para desenvolvimento de aplicações com Web semântica em Java, provendo suporte para os padrões RDF e RDFS, assim como para as linguagens OWL e SPARQL (Martimiano, 2006). O Jena teve início no laboratório de pesquisa sobre Web Semântica da HP (Hewlett-Packard) ${ }^{13}$, está escrito em Java e teve seu código aberto à comunidade open source ${ }^{14}$.

As recomendações de Web semântica para RDF, RDFS e OWL têm como núcleo o grafo RDF (Tripla: Sujeito, Predicado, Objeto). Da mesma forma, o Jena ${ }^{15}$ é igualmente centrado no grafo RDF. O RDFS e o OWL são vistos como transformações grafo a grafo, produzindo grafos de tripla virtual (Carroll et al., 2004).

O Jena fornece uma API (Application Programming Interface) para criação e manipulação desses grafos. Essa API possui classes de objetos para representar grafos, recursos, propriedades e literais. As interfaces representando recursos, propriedades e literais são denominadas Resource, Property e Literal, respectivamente. No Jena, um grafo é chamado de modelo, e é formado por um conjunto de triplas (Statements), sendo representado pela interface Model.

A arquitetura do Jena, conforme mostrada na Figura 5.1, é dividida em três camadas (Carroll et al., 2004):

\footnotetext{
${ }^{12}$ O UDDI é uma especificação elaborada pela OASIS (http://www.oasis-open.org)

${ }^{13} \mathrm{http}: / /$ www.hpl.hp.com/semweb/

${ }^{14} \mathrm{http}: / / \mathrm{www} . o p e n j e n a . o r g /$

${ }^{15}$ Referenciado internamente na HP como Jena2 (segunda versão do Jena).
} 
- Graph Layer - Camada baseada na sintaxe abstrata do RDF, fazendo uso de triplas como a estrutura de dados universal (Triples as the Universal Data Structure). Esta camada simplifica a implementação:

- Do armazenamento de triplas, tanto em memória como em um armazenamento persistente ${ }^{16}$.

- De visões como triplas em somente leitura (read-only) dos dados que não são triplas, por exemplo, dados lidos a partir de uma hierarquia de um sistema de arquivos, ou de uma página da Web.

- De triplas virtuais correspondentes aos resultados dos processos de inferência sobre algum outro conjunto de triplas como premissas.

- Model Layer - Camada que mantém a Model API como a principal abstração do grafo RDF; essa API é utilizada pelo programador da aplicação, fornecendo um conjunto mais rico de métodos para as operações com os grafos (Model interface) e com os nós (Resource interface). Além disso, a API DAML forma uma API de Ontologia (Ontology API).

- EnhGraph Layer - Camada que fornece um ponto de extensão para prover visões de grafos e nós para criação de APIs. Permite múltiplas visões de grafos e nós que podem ser utilizados simultaneamente.

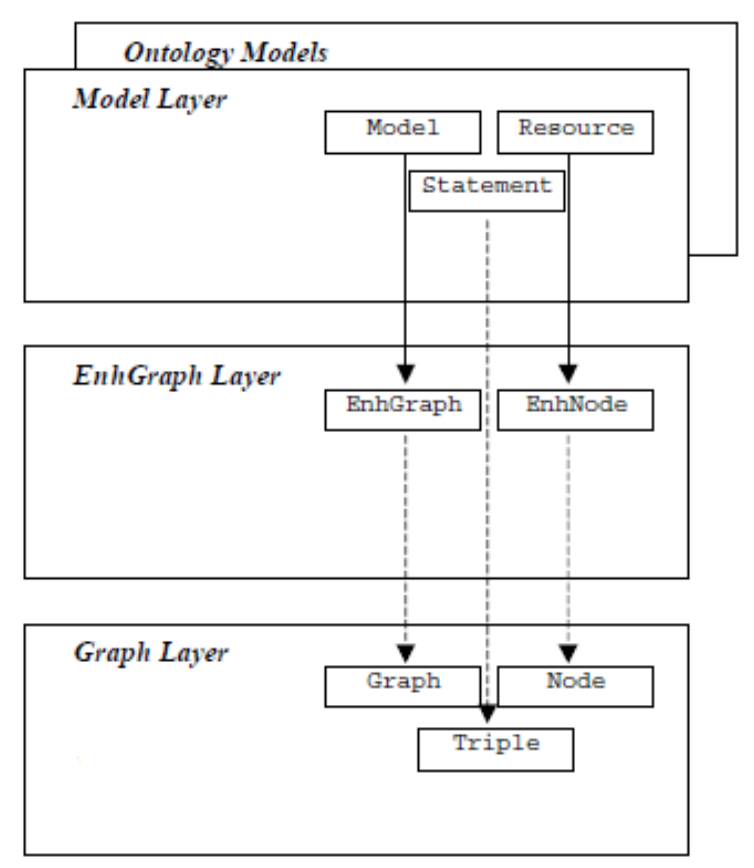

Figura 5.1: Arquitetura do Jena (Carroll et al., 2004).

Para a manipulação de Web Semântica com ontologias, o Jena possui uma API denominada Ontology API. Essa API inclui suporte para trabalhar com ontologias em linguagens como RDFS

\footnotetext{
${ }^{16} \mathrm{O}$ Jena oferece suporte aos SGBDs (Sistema Gerenciador de Base de Dados): MySql, Oracle e PostgreSQL.
} 
e OWL, sendo que cada linguagem tem um perfil (profile), o qual lista as construções permitidas e as URIs das classes e propriedades. O perfil está vinculado a um modelo de ontologia, que é uma versão do modelo de classe do Jena estendido. O modelo geral permite acesso às declarações nas coleções de dados do RDF. A interface OntModel estende esse modelo geral e adiciona suporte para os tipos de objetos esperados em uma ontologia como, por exemplo, classes (em uma hierarquia de classes), propriedades (em uma hierarquia de propriedades) e indivíduos (instâncias de uma ou mais classes) (Carroll et al., 2004).

Portanto, o Jena permite aos desenvolvedores manipular e criar os dados semânticos programaticamente, baseados no grafo RDF e com extensão para a linguagem OWL-DL, a qual foi utilizada no desenvolvimento da ontologia do UDOnt-Q. Essas características influenciaram na escolha por usar esse framework neste trabalho, na criação de algoritmos para realizar a manipulação da ontologia criada no Protégé. Duas versões foram utilizadas para a criação de dois algoritmos:

- A versão 2.3, pois, disponibiliza a linguagem de consulta RDQL (RDF Data Query Language) criada para extrair informações de grafos RDF. Essa consulta trata o grafo RDF como dado, puramente, não fazendo distinção entre triplas básicas e inferidas (Seaborne, 2004a). Dessa forma, a linguagem de consulta RDQL foi utilizada para a criação de um algoritmo de busca do módulo. Contudo, segundo o próprio Seaborne (2004), os novos programadores devem utilizar a linguagem de consulta SPARQL por ser mais recente e sofisticada que a RDQL (Seaborne, 2004b). Assim, a linguagem SPARQL-DL foi utilizada neste trabalho.

- A versão 2.6.3 foi utilizada como padrão no desenvolvimento do algoritmo de busca que utiliza as classes disponíveis na API do Jena. Mais detalhes sobre os algoritmos de busca por Web Services com QoS são apresentados na próxima seção deste capítulo.

\subsection{Componentes e Estrutura}

O Módulo UDOnt-Q foi criado com o intuito de servir de base para os algoritmos de busca e seleção semântica de Web Services com QoS. As informações sobre os acordos entre clientes e provedores, bem como a qualidade de serviço e seus atributos estão em uma base de conhecimento que está representada pela ontologia discutida anteriormente no Capítulo 4.

Este módulo foi projetado para ser reutilizável e configurável aceitando novos algoritmos (não apenas semânticos) exigindo o mínimo de alterações em código-fonte. Ele foi desenvolvido com a linguagem de programação Java para que possa ser portável para diversas plataformas.

O UDOnt-Q está divido em componentes (pacotes - java packages), sendo que cada componente executa uma determinada função dentro do módulo e alguns pacotes possuem dependências entre si. Ao final do desenvolvimento de um componente, o mesmo foi submetido a testes unitários visando a minimizar a ocorrência de erros. O módulo é composto por cinco componentes: Util, Command Components (CC), UDDI Components (UC), Ontology Components (OC) e Common Information Shared (CIS). 


\subsubsection{Componente Util}

O componente Util (Figura 5.2) é composto por classes "úteis" que são utilizadas por outras classes do módulo para realizar tarefas úteis e necessárias. Por exemplo, foram desenvolvidos mecanismos de logs para registrar erros e informações em arquivos no disco (para isso, este componente utilizou a ferramenta $\log 4 \mathrm{~J}$ ). Também foi criado outro mecanismo com a função de registrar resultados de desempenho em um banco de dados (mySQL).

Toda a parte de configuração do módulo é feita neste componente, por meio da Classe "Config", que herda o conceito de propriedades da classe "java.util.Properties". Dessa forma, um arquivo de configuração pode ser utilizado pelo módulo para armazenar e recuperar informações tais como: endereço de conexão com o banco de dados, localização do arquivo da ontologia, endereço, usuário e senha para acesso no registro UDDI e qual algoritmo de busca o módulo deve utilizar. Além disso, estão presentes diversos métodos úteis como, por exemplo, métodos para o tratamento de strings.

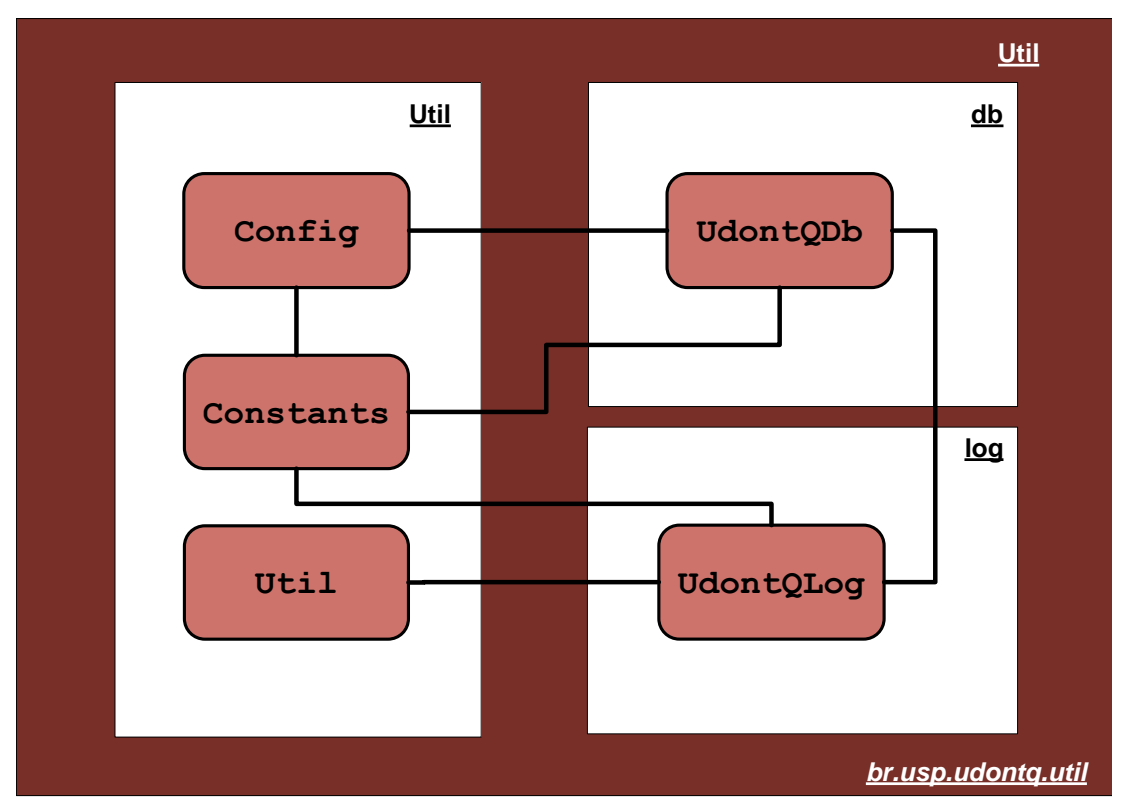

Figura 5.2: Componente Util.

\subsubsection{Componente Command Components (CC)}

O componente Command Components (CC) (Figura 5.3) contém classes criadas para comandar, analisar e direcionar as requisições dos clientes, para que sejam atendidas por outras classes de outros componentes do módulo. As configurações carregadas na inicialização do módulo são utilizadas pelas classes deste componente a fim de instância os componentes e classes adequados.

Este componente possui um pacote (package) que constitui um sub-componente denominado QoS Components (QC) (Figura 5.4). A função do QoS Components é auxiliar o Command Components na análise da qualidade dos serviços dos provedores. Em caso de um empate en- 


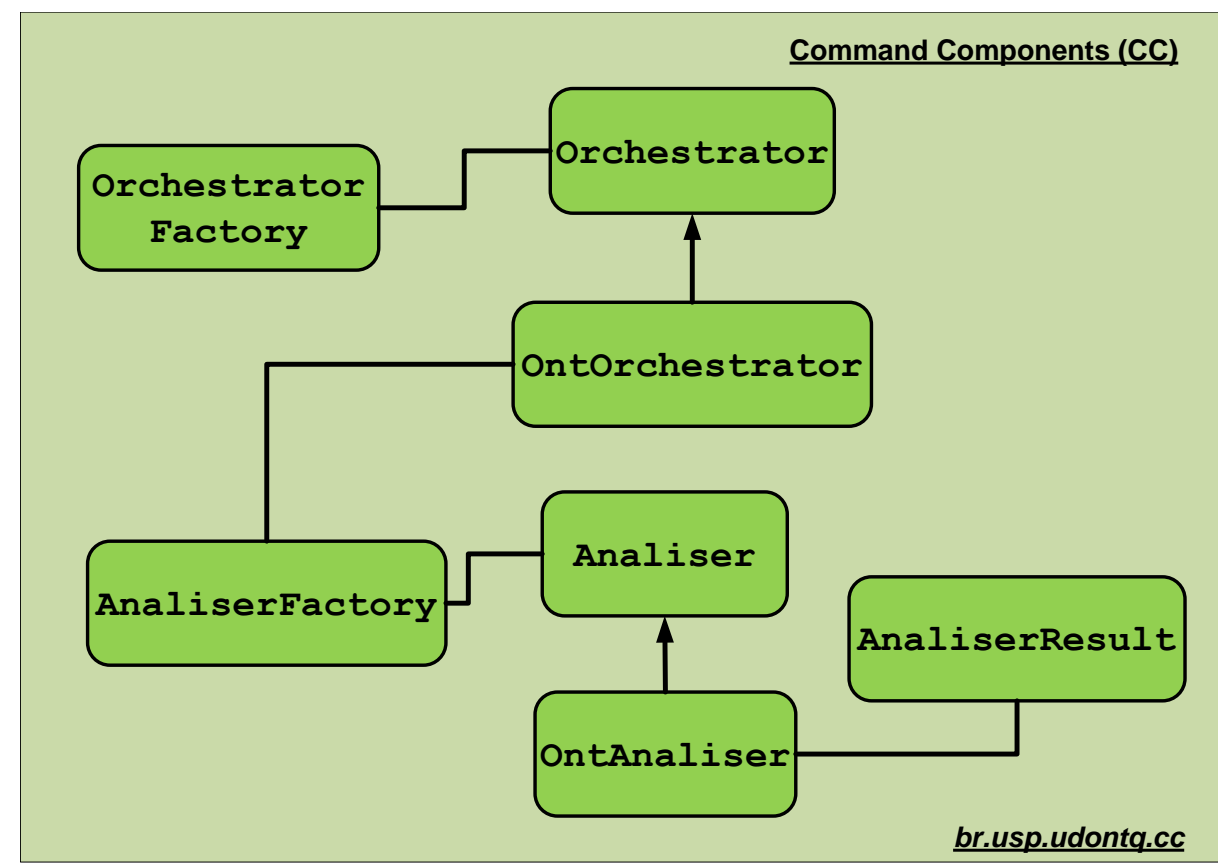

Figura 5.3: Componente Command Components (CC).

tre serviços de mesma QoS, a implementação da classe abstrata QoSInformer irá decidir qual é o melhor serviço baseado em informações constantemente recolhidas dos provedores de serviços por um monitor. Neste projeto foi utilizado o monitor de sistemas Ganglia, portanto a classe concreta QoSInformerGanglia foi implementada e utilizada. Porém, outras classes podem ser adicionadas ao QC para utilizar outros monitores exigindo simples modificações no código-fonte.

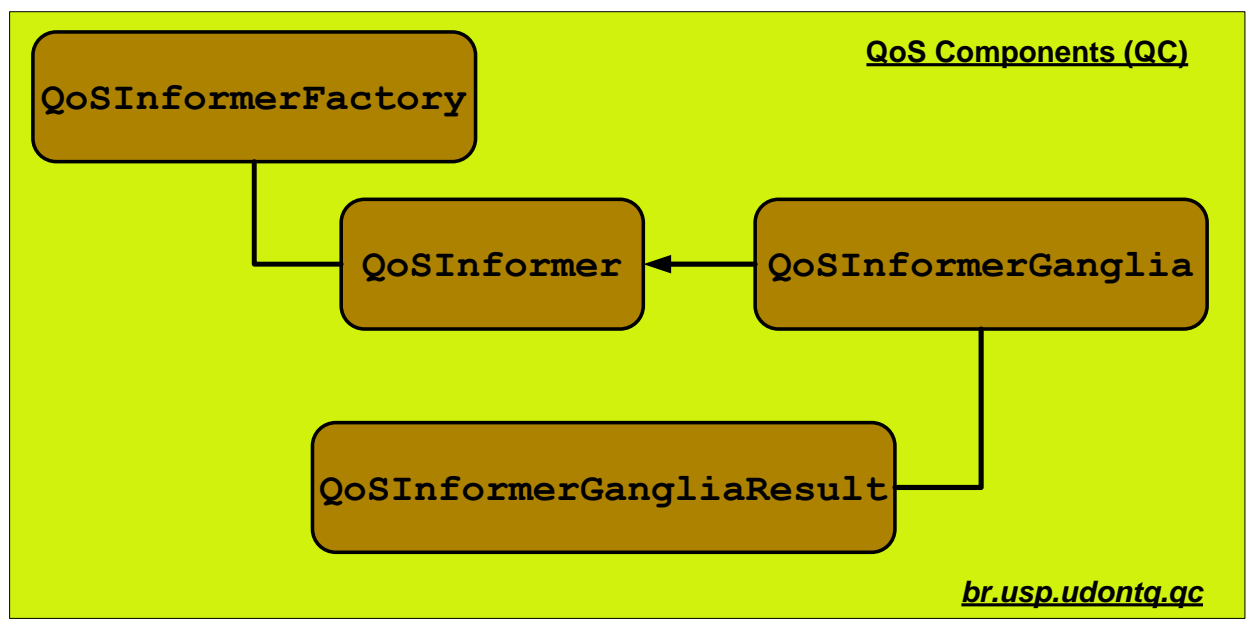

Figura 5.4: Sub-componente $Q o S$ Components $(Q C)$.

Para a escolha do melhor serviço são consideradas informações sobre a carga da CPU e a utilização de memória nos provedores de serviços. O provedor escolhido será aquele que, naquele momento, encontra-se menos carregado, conforme a Equação (5.1):

$$
P C=(100-P C P U I D L E) * 3+\left(100-\frac{M E N F R E E * 100}{M E N T O T A L}\right) * 2
$$


Onde:

- PC é o percentual de carga.

- PCPUIDLE é o percentual de CPU ociosa.

- MENFREE é a quantidade de memória RAM livre.

- MENTOTAL é a quantidade total de memória no provedor.

Dessa forma observa-se que, de acordo com a Equação 1, a porcentagem de CPU ocupada (100 - CPU Ociosa) tem peso 3 e a de memória ocupada (100 - regra de três para obter a porcentagem de memória livre) tem peso 2. O provedor que tiver o menor PC será escolhido, em caso de um novo empate o primeiro provedor da lista será considerado.

\subsubsection{Componente UDDI Components (UC)}

O componente $\boldsymbol{U D D I}$ Components $(\boldsymbol{U C})$ (Figura 5.5) reúne as classes com a função de prover o acesso a informações no registro UDDI. Também permite que novos Web Services e provedores sejam cadastrados no registro.

A classe principal desenvolvida foi a UddiRequest. Ela recebe as requisições feitas ao registro UDDI e por meio da classe abstrata UddiAbstration são instanciadas classes de manutenção do registro UDDI de acordo com a sua implementação especificada no arquivo de configuração. Para este projeto foi desenvolvida apenas a classe JuddiImpl que possui métodos de interatividade com a implementação do registro UDDI da Apache Software Foundation, denominado jUDDI (java UDDI). Algumas classes do pacote jUDDI foram utilizadas para facilitar a interação com esse registro. Outras classes para interagir com outras implementações de outros registros UDDI podem ser facilmente adicionadas a este sistema, exigindo-se o mínimo de alteração no código-fonte.

\subsubsection{Componente Ontology Components (OC)}

O componente Ontology Components (OC) (Figura 5.6) contém as classes de algoritmos para a busca semântica por Web Services com QoS. A busca pode ser executada por meio de três algoritmos:

- OntAlgorithmObject: Este algoritmo realiza a busca utilizando as classes fornecidas pelo Jena. Ele cria instâncias de objetos com as informações da ontologia. Dessa forma, a pesquisa é feita por meio de interações (loops) na lista de objetos até que se encontrem os clientes, serviços, provedores e QoS adequados. O algoritmo recebe dois parâmetros de entrada que são o endereço IP do cliente e o nome do Web Service que ele desejada. Com 


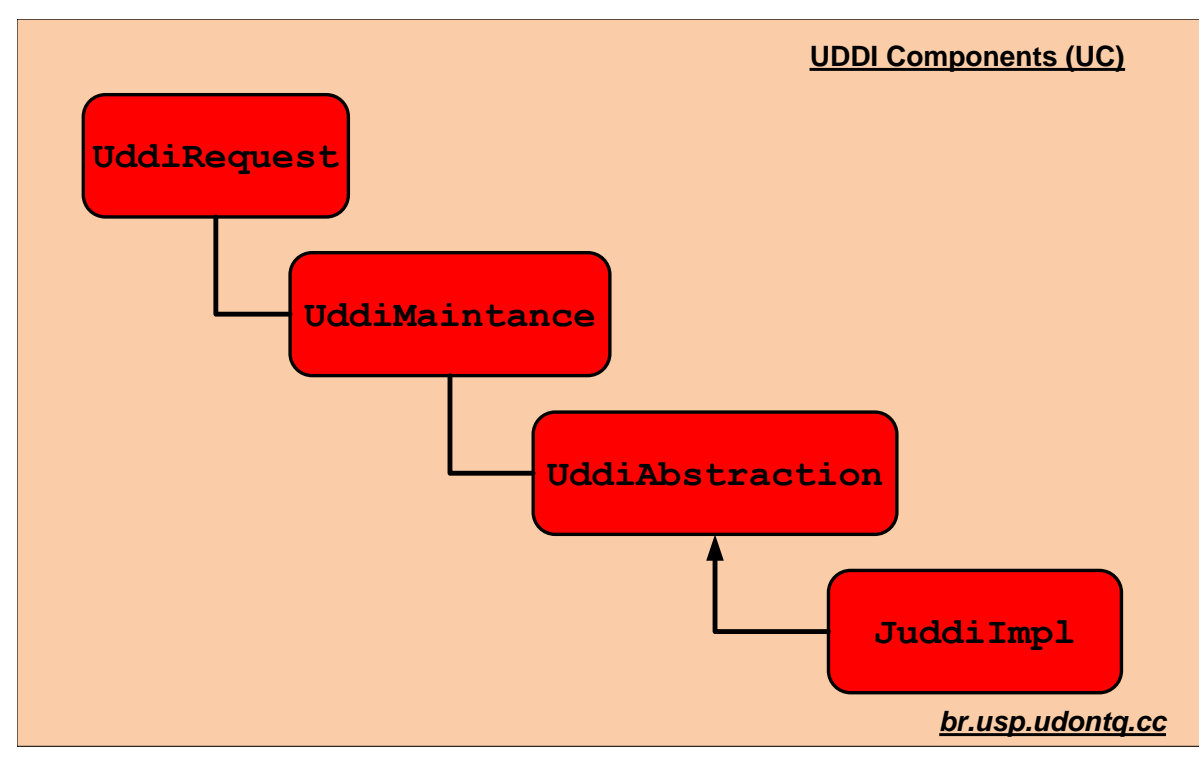

Figura 5.5: Componente UDDI Components $(U C)$.

o endereço IP o algoritmo localiza os dados do cliente na ontologia e logo em seguinda indentifica também qual é a sua classe (ClientGold, ClientSilver ou ClientBronze). De acordo com a classe do cliente, o algoritmo seleciona apenas os serviços com a QoS correspondente e que tenham o mesmo nome que foi passado como parâmetro de entrada. Dessa forma, as respostas são inseridas em uma estrutura de dados (Web Service, Descrição do Provedor Endereço IP do Provedor) que é retornada ao módulo.

- OntAlgorithmRDQL: utiliza a linguagem de consulta RDQL para consultar informações na ontologia, opera com algumas semelhanças a uma consulta SQL (Structured Query Language). O funcionamento desse algoritmo é semelhante ao do OntAlgorithmObject, porém a busca é feita por uma query que considera os parâmetros de entrada como filtros na cláusula where.

- OntAlgorithmSPARQL: este algoritmo faz uso do pacote que consulta o Pellet e que permite a utilização da linguagem de consulta SPARQL-DL para consultar informações na ontologia. O funcionamento desse algoritmo é semelhante ao do OntAlgorithmRDQL, porém há diferenças nos recursos utilizados e na sintaxe da query de consulta.

É importante enfatizar que o módulo é flexível e permite a troca de um algoritmo por outro sem a necessidade de alterações no código. Além disso, novos algoritmos podem ser desenvolvidos e facilmente adicionados ao pacote, pois, foram utilizados alguns padrões de desenvolvimento e primitivas da linguagem Java (instância única (singleton), fábricas, interfaces e classes abstratas) durante a construção de todo o módulo. 


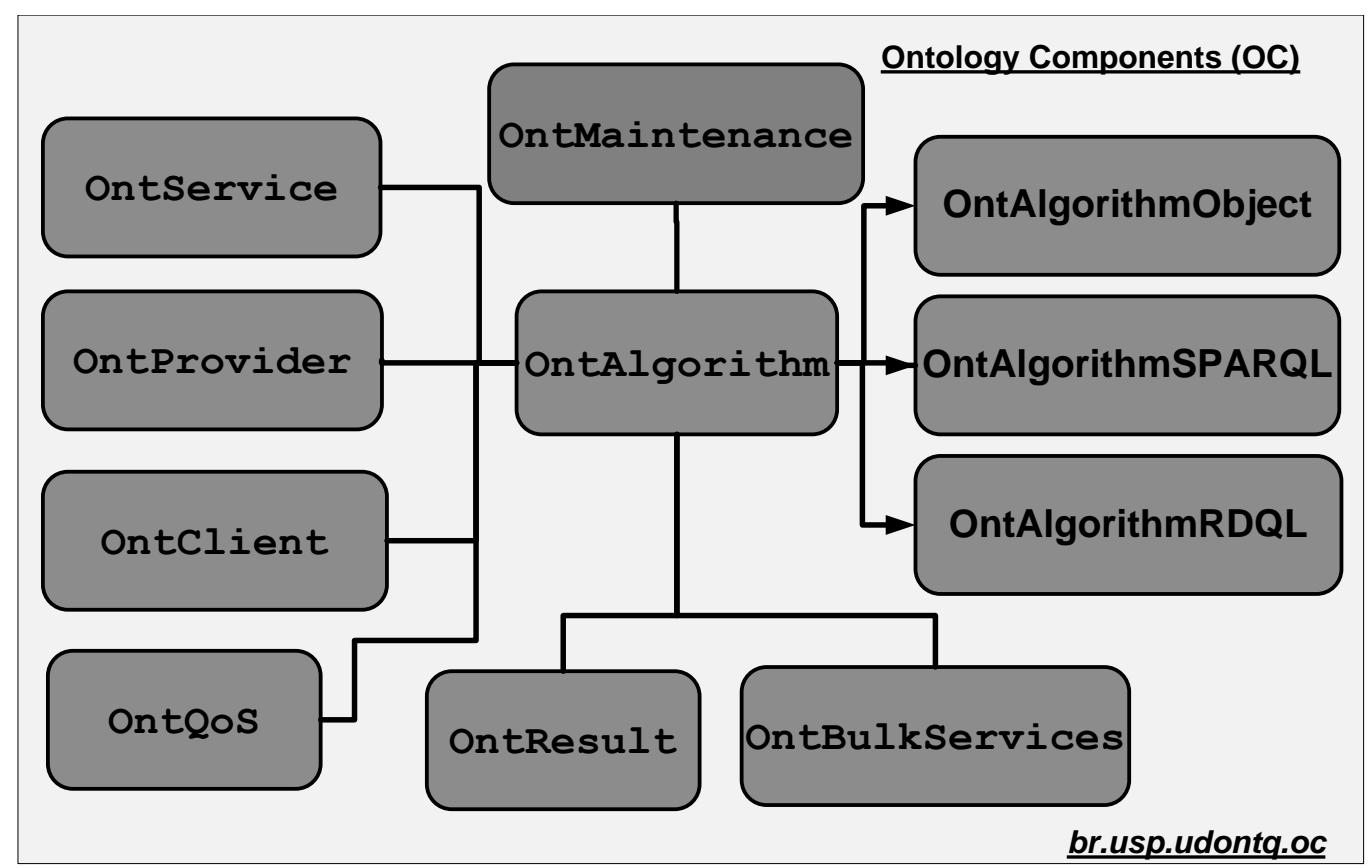

Figura 5.6: Componente Ontology Components (OC).

\subsubsection{Componente Common Information Shared (CIS)}

O componente Common Information Shared (CIS) (Figura 5.7) provê uma classe com métodos para a manutenção das informações na ontologia e, consequentemente, no registro UDDI. Por exemplo, caso um provedor disponibilize um novo serviço ele deve cadastrá-lo na ontologia e também no registro UDDI. Por meio dos métodos dessa classe é possível realizar as duas tarefas de forma conjunta.

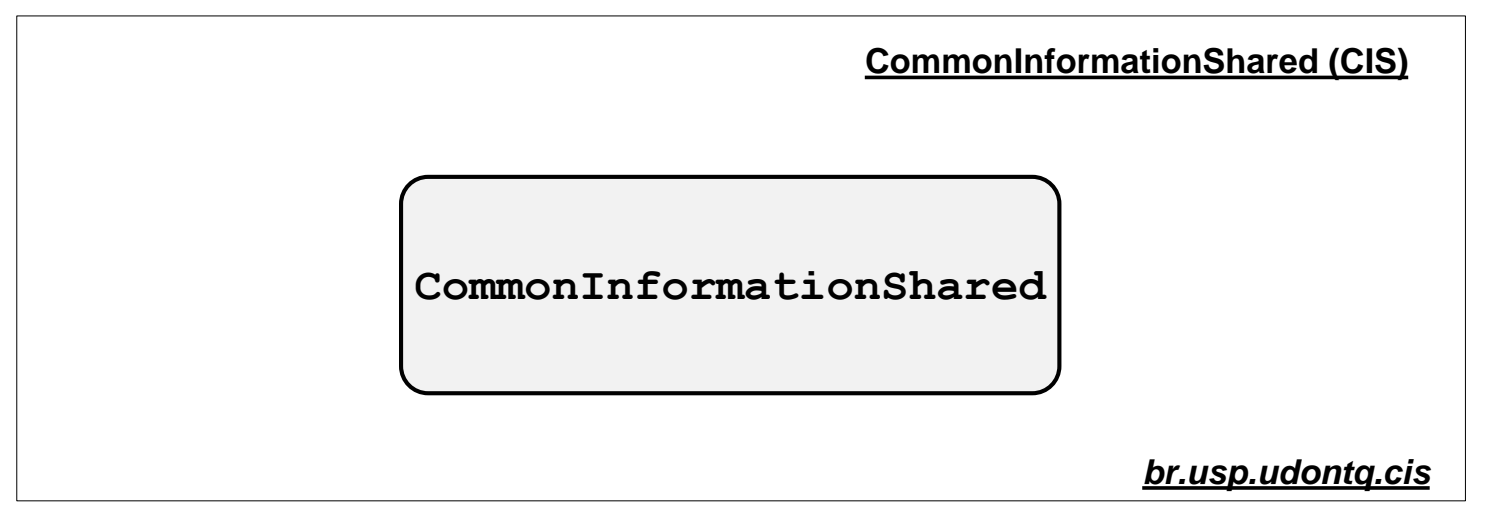

Figura 5.7: Componente Common Information Shared (CIS).

\subsubsection{Estrutura dos Componentes do UDOnt-Q}

Os componentes do UDOnt-Q formam um conjunto de funcionalidades que precisam ser utilizados (importadas) por uma classe principal que seja executável. Esse software executável deve estar disponível para os clientes que buscam os Web Services com QoS. Das diversas possibilidades 
de acesso que poderiam ser utilizadas pelos clientes para acessar o módulo (Socket, RMI (Remote Method Invocation), etc.), optou-se por transformar o módulo em um Web Service para garantir a interoperabilidade de acesso pelos mais diversos clientes.

O Web Service criado possui uma operação (método) denominada GetWSDL na qual o cliente passa como parâmetro apenas o nome do serviço que deseja. Contudo, esse método foi sobrecarregado (overloading) para a inclusão de um parâmetro com o endereço de Internet (IP). No primeiro método não é preciso passar o IP do cliente como parâmetro, pois, esse endereço é recuperado do contexto da mensagem de conexão HTTP. Porém, a sobrecarga do método foi necessária porque durante os testes realizados uma máquina cliente, por meio de threads, simula ser vários clientes que executam requisições concorrentes ao módulo.

A Figura 5.8 exibe resumidamente a estrutura completa do módulo como um Web Service sendo consumido por um cliente, enquanto monitora diversos provedores (Nakamura et al., 2011a).

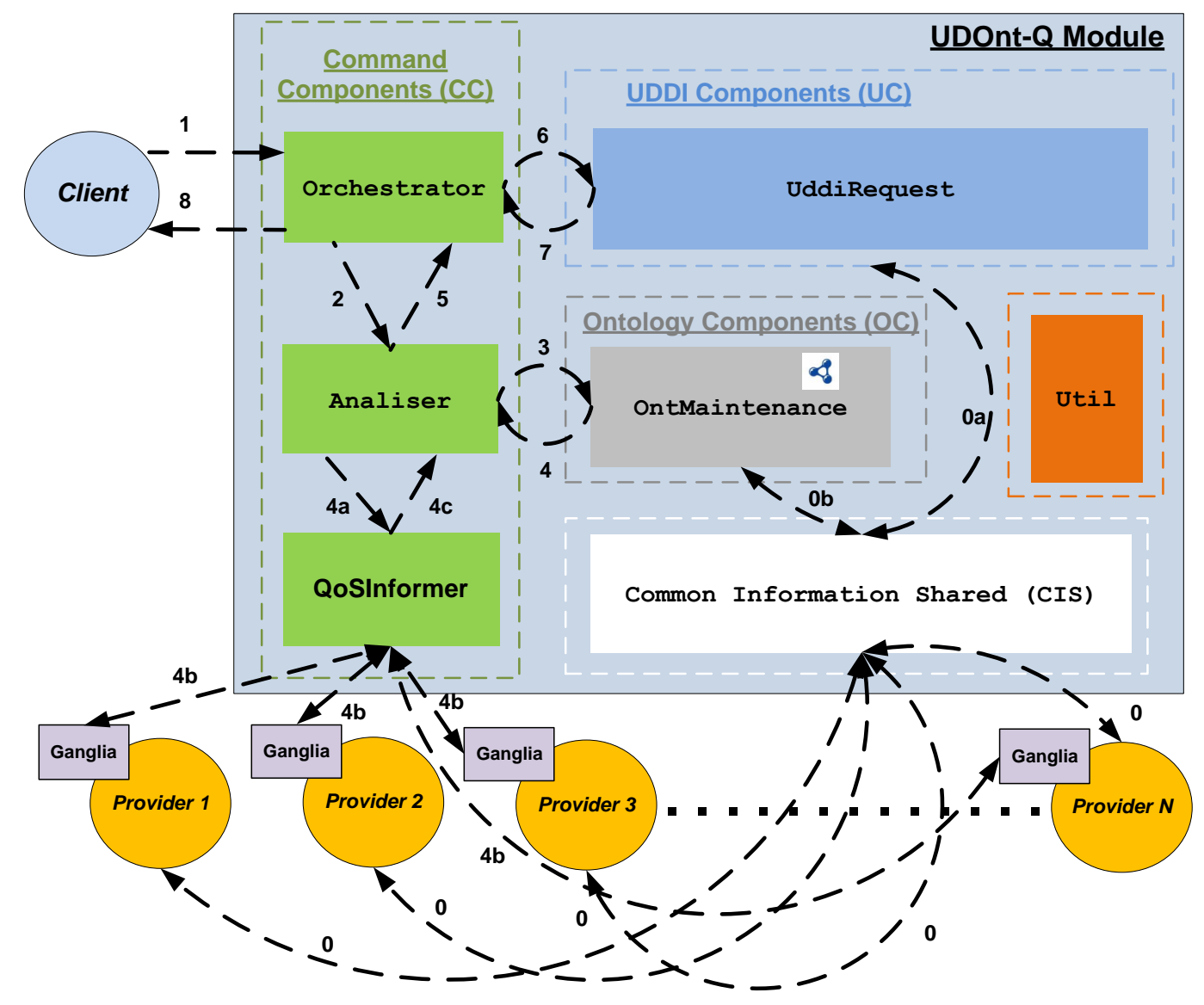

Figura 5.8: Estrutura resumida do Módulo com os seus componentes (Nakamura et. al, 2011a).

A sequencia do funcionamento do UDOnt-Q também é exibida na Figura 5.8. Tal funcionamento é dado pelos seguintes passos (Nakamura et al., 2011a):

- 0. A princípio os provedores de serviços devem implementar a classe do componente CIS para que possam registrar os seus serviços tanto no registro UDDI (0a), como na ontologia (0b). 
- 1. O módulo é fornecido como um Web Service que é consumido pelo cliente que informa o nome do serviço desejado. Opcionalmente o endereço IP do cliente também poderá ser fornecido, caso contrário o mesmo é recuperado pelo contexto da mensagem de conexão HTTP.

- 2. O componente $\mathbf{C C}$ encaminha a requisição para a análise a fim de verificar qual componente de busca será utilizado. Neste trabalho, o componente desenvolvido para realizar a busca é o OC, porém outros componentes podem ser desenvolvidos e acoplados facilmente ao módulo.

- 3. O componente $\mathbf{O C}$ recebe as informações e instancia o algoritmo de busca (por exemplo: OntAlgoritmObject ou OntAlgoritmSPARQL) que está indicado no arquivo de configuração do módulo. Dessa forma, baseando-se no acordo determina-se a QoS contratada pelo cliente e também quais serviços atendem aos requisitos dessa QoS. O resultado da busca é um conjunto de dados composto pelo: o nome do serviço, o nome do provedor e o endereço do provedor.

- 4. O CC recebe e analisa o resultado da busca feita pelo algoritmo. A análise consiste em verificar se há um ou mais serviços que atendam à requisição do cliente. Existindo apenas um, esse serviço é retornado à classe Orchestrator e o sistema continuará o fluxo (item 5). Caso haja mais de um registro, o sistema pode seguir dois fluxos que dependem da opção de monitoramento indicada no arquivo de configuração. Caso a opção esteja desativada (disabled), o primeiro resultado da lista será retornado. Porém, caso a opção esteja ativa (enabled) a lista de resultados é encaminhada para o sub-componente QC. A classe QoSInformer analisará os estados atuais dos provedores que estavam presentes na lista de resultados (4a, 4b e 4c) e irá escolher aquele que estiver menos sobrecarregado.

- 5. A classe Orchestrator recebe o resultado, recupera o nome do serviço e do provedor e encaminha para o componente $\mathbf{U C}$.

- 6. O componente UC procede com a pesquisa no registro UDDI, procurando a WSDL do serviço daquele determinado provedor.

- 7. A WSDL é retornada ao componente $\mathbf{C C}$ que a repassa ao cliente.

- 8. O cliente recebe a WSDL do serviço que possui a qualidade contratada e a partir dela é possível determinar a localização do serviço e posteriormente utilizá-lo. A resposta Null é enviada ao cliente caso nenhum serviço que atenda a sua necessidade seja encontrado.

Durante todo o fluxo da Figura 5.8, eventuais erros são registrados e informações de desempenho são coletadas para que uma futura avaliação de desempenho seja conduzida. 


\subsection{Considerações Finais}

Neste capítulo foi apresentado o desenvolvimento do módulo UDOnt-Q. A metodologia e ferramentas utilizadas no processo de desenvolvimento do módulo, juntamente com os componentes, a estrutura e o funcionamento do módulo, foram tópicos abordados neste capitulo que permitiram o seu melhor entendimento. Após as correções de alguns erros encontrados durante a fase de testes, o protótipo do módulo ficou pronto para ser submetido a uma avaliação de desempenho. A avaliação de desempenho do módulo e de seus algoritmos é discutida no próximo capítulo. 


\subsection{Considerações Iniciais}

Este capítulo aborda a avaliação de desempenho do protótipo do UDOnt-Q. Essa avaliação considera os algoritmos de busca OntAlgoritmObject e OntAlgoritmSPARQL durante os experimentos. O objetivo deste capítulo é analisar o comportamento do módulo quando submetido a diversas requisições concorrentes. Algumas variáveis de respostas são analisadas, como por exemplo, o tempo para a localização de serviços e a vazão do módulo, o tempo de resposta para o cliente obter a descrição de interface do serviço (WSDL) e a sobrecarga de processamento causada pela utilização do módulo em algumas situações.

\subsection{Configuração do Ambiente de Testes}

A configuração do ambiente de testes detalha os elementos de hardware e software utilizados nos experimentos. Tais informações são importantes porque facilitam a reprodução do ambiente utilizado durante os testes. Além disso, essas informações visam a impedir comparações incorretas, pois em alguns casos uma simples alteração pode fornecer resultados completamente diferentes. Nesse contexto, na Tabela 6.1 é possível visualizar a infraestrutura computacional utilizada na avaliação de desempenho do protótipo do módulo UDOnt-Q.

Complementando os elementos de hardware descritos na Tabela 6.1, a Tabela 6.2 lista os principais elementos de software utilizados no desenvolvimento e também na avaliação de desempenho do módulo. 
Tabela 6.1: Elementos de Hardware

\begin{tabular}{|c|c|c|c|}
\hline Componente & Quantidade & Configuração & Descrição \\
\hline $\begin{array}{l}\text { Provedores de } \\
\text { Serviços }\end{array}$ & 5 & $\begin{array}{c}\text { Intel QuadCore Q6000 }(2.4 \mathrm{GHz}) \\
\text { 2GB de RAM } \\
\text { HD 120GB, 7200RPM }\end{array}$ & $\begin{array}{c}\text { Fornecer os } \\
\text { Web Services }\end{array}$ \\
\hline Clientes & 3 & $\begin{array}{c}\text { Intel QuadCore Q9400(2.66GHz) } \\
\text { 4GB de RAM } \\
\text { HD 500GB, 7200RPM }\end{array}$ & $\begin{array}{l}\text { Requisitar os Web } \\
\text { Services com QoS }\end{array}$ \\
\hline UDOnt-Q & 1 & $\begin{array}{c}\text { Intel QuadCore Q9400(2.66GHz) } \\
\text { 8GB de RAM }\end{array}$ & Módulo de busca \\
\hline UDDI & & HD 320GB, 7200RPM & Registro de Informações \\
\hline
\end{tabular}

Tabela 6.2: Elementos de Software

\begin{tabular}{|c|c|c|c|}
\hline $\begin{array}{c}\text { Elemento } \\
\text { Server }\end{array}$ & Descrição & Utilização & Versão \\
\hline $\begin{array}{c}\text { Apache Web } \\
\text { Server }\end{array}$ & $\begin{array}{c}\text { Servidor Web } \\
\text { da Apache }\end{array}$ & $\begin{array}{c}\text { Armazenamento e } \\
\text { disponibilização da } \\
\text { ontologia via URL }\end{array}$ & $\mathbf{2 . 2 . 1 4}$ \\
Kernel 2.6.32-26
\end{tabular}

Além dos elementos de software citados na Tabela 6.2, é importante salientar que também foram utilizados: o módulo com os componentes desenvolvidos neste projeto, as aplicações clientes e uma aplicação para geração de carga de trabalho nos provedores de serviço. Essa última aplicação, denominada ProviderWorkLoad, foi criada com o intuito de gerar carga de trabalho do tipo 
$C P U-B o u n d$, não permitindo que a CPU fique ociosa durante os experimentos. Ela é composta por operações matemáticas (exponenciais e divisões) dentro de laços aninhados na ordem $O\left(n^{4}\right)$. Essas operações são executadas de tempos em tempos de forma aleatória, as variáveis que representam as bases e os expoentes também são geradas aleatoriamente dentro de um limite. Dessa forma, espera-se que a carga de trabalho dos provedores não seja a mesma, evitando que o módulo sempre escolha o mesmo provedor.

A Figura 6.1 resume a disposição dos softwares utilizados no ambiente de testes. A seta na cor Preta representa a interação do cliente com o módulo ao fazer a sua requisição. A seta na cor Verde indica a consulta do módulo a uma URL (ontologia) no servidor Web Apache. A gravação de $\log s$ em disco é feita com a utilização do $\log 4 \mathbf{4}$. Para a gravação dos resultados de desempenho foram desenvolvidas e executadas stored procedures, gravando assim os dados no banco de dados MySql (seta Marrom).

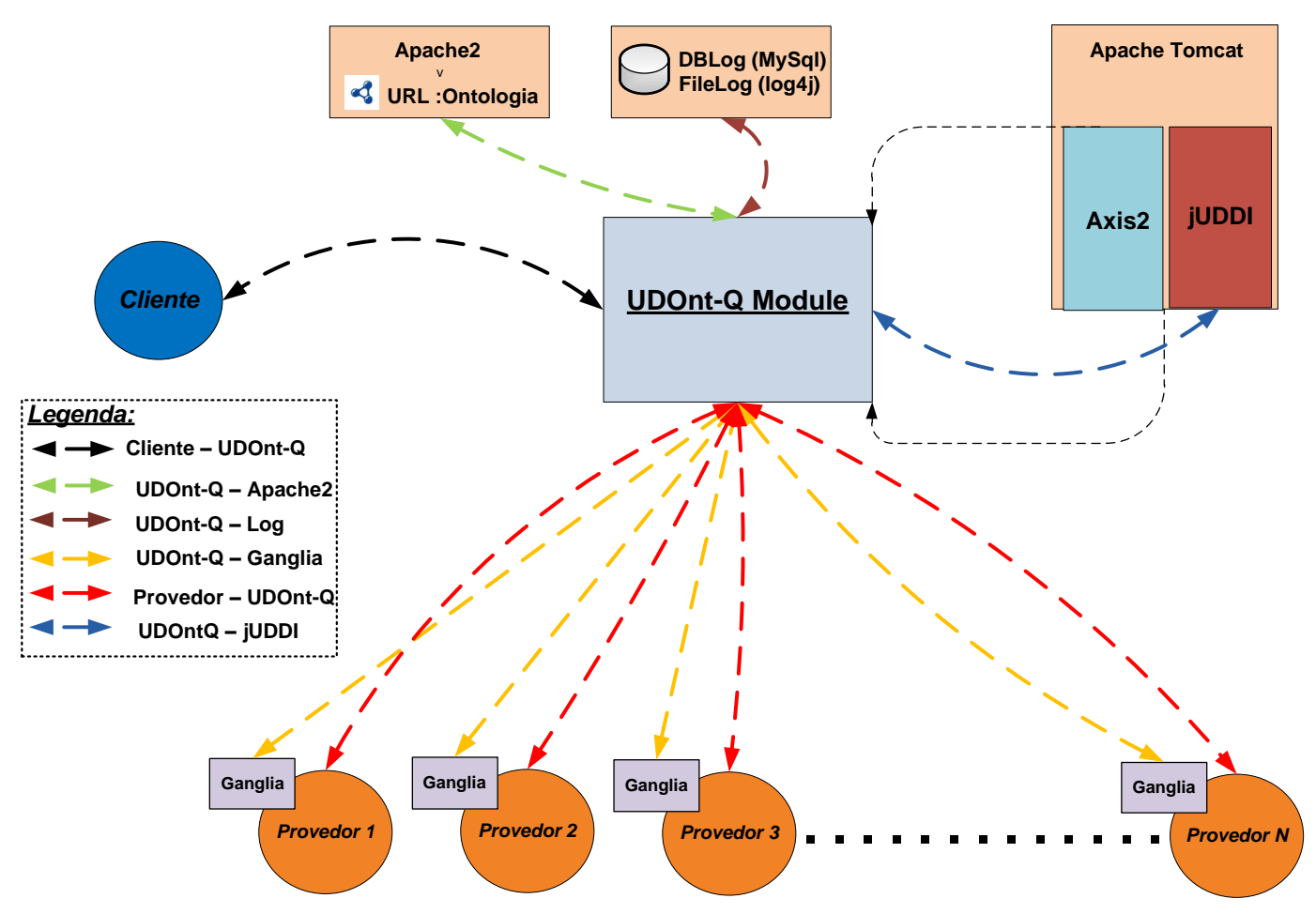

Figura 6.1: Visão resumida dos elementos de software utilizados no ambiente de testes.

Quando a funcionalidade de monitoramento dos provedores está ativa e há mais de um serviço como resultado, o módulo passa a considerar as informações fornecidas pelo monitor Ganglia. A interação do módulo com o Ganglia está representada pelas setas na cor Amarela. Os provedores podem atualizar a ontologia (e o registro UDDI) por meio de métodos da classe do componente CIS. As setas Vermelhas esboçam esta interação. É importante relembrar que o módulo é um conjunto de componentes que tem um Web Service como interface de acesso e, por este motivo, o módulo precisa ser implantado (deploy) em um contêiner de Servlets (Apache Tomcat) onde poderá 
ser acessado pelos clientes. O Axis2 é exigido, pois este Web Service utiliza o protocolo SOAP para a troca de informações. Por fim, a seta na cor Azul representa a comunicação entre o módulo e o jUDDI na consulta de dados funcionais (WSDL). O jUDDI, o Axis2 e consequentemente o módulo são aplicações que para seu funcionamento precisam estar inseridas em um servidor de aplicações que neste caso é o Apache Tomcat.

\subsection{Planejamento de Experimentos}

Antes de iniciar o processo de avaliação de desempenho do módulo foi realizado o planejamento de experimentos. O planejamento tem como objetivo obter o máximo de informações com um número mínimo de experimentos (Jain, 1991), constituindo uma etapa fundamental em que é preciso definir quais são as informações e em que condições e quantidade elas devem ser coletadas em um determinado experimento (Estrella, 2010).

O planejamento de experimentos pode facilitar o entendimento do comportamento de desempenho do módulo em determinadas situações. Durante o planejamento de experimentos buscam-se identificar quais serão as variáveis de respostas do sistema que deverão ser analisadas; por exemplo, se o tempo de resposta é importante então ele deve ser considerado como uma variável de resposta e, portanto, deve ser coletado durante os experimentos.

Outro ponto que deve ser identificado é o que será avaliado, quais os fatores que influenciam no desempenho do sistema e quais níveis desses fatores podem ser interessantes. Por exemplo, o fator "número de usuários de um sistema" é interessante e as diversas quantidades de usuários são os níveis desse fator. Além disso, pode haver fatores de menor influência no sistema, chamados de fatores secundários. É interessante também informar o número de vezes que os experimentos são repetidos, ou seja, "replicados", pois uma única replicação pode estar sujeita a interferências que podem não ser comum no ambiente analisado (Jain, 1991).

Após analisar a influência de cada fator, também é possível verificar a interação entre eles. $\mathrm{O}$ resultado dessas interações é interessante, pois algumas vezes um fator isolado não exerce uma influência significativa, porém quando combinado a outro resulta em uma interação onde a sua influência é considerável no sistema avaliado.

Segundo Jain (1991), das diversas técnicas existentes para realizar o planejamento de experimentos as mais utilizadas são: o planejamento simples, o planejamento fatorial completo e o planejamento fatorial parcial. A técnica adotada neste projeto foi o planejamento fatorial completo, que utiliza todas as combinações possíveis de todos os fatores e níveis escolhidos para a avaliação. Essa técnica é mais trabalhosa que as demais, contudo, permite que os fatores sejam avaliados, sendo assim possível identificar as interações e influências entre eles (Jain, 1991).

Um fato importante ocorreu durante a execução dos experimentos do módulo UDOnt-Q. Notouse uma característica interessante de comportamento do processo de classificação e realização (inferência) da ontologia por parte do reasoner Pellet. Quando foram adicionados mais indivíduos 
na ontologia, maiores foram os tempos de processamento para a classificação e realização (inferência). Esse aumento já era esperado, pois haveria mais informações a serem analisadas, porém a intensidade do aumento não parecia seguir um comportamento linear.

Sendo assim, decidiu-se avaliar também esse processo de classificação e realização (inferência) da ontologia. Esse fato passou despercebido durante a fase de testes do módulo devido ao pequeno número de indivíduos na ontologia utilizada para os testes unitários. Esse problema foi detectado quando se adotou o número de serviços cadastrados como um fator para os experimentos. Além disso, estabeleceu-se a dúvida se esse comportamento ocorria devido às características da ontologia criada. A fim de sanar essa dúvida, foi escolhida uma ontologia clássica para analisar o seu comportamento com vários indivíduos. A ontologia Pizza, comentada na seção 4.3, inspirou a criação da ontologia para o módulo e foi escolhida para a comparação. Dessa forma, foram criados três Planejamentos de Experimentos (PE) para este trabalho:

- Planejamento de Experimentos para a avaliação de desempenho do processo de classificação e realização (inferência) da ontologia do módulo utilizando o processo de inicialização do módulo com a configuração padrão do reasoner Pellet (PE-Ontologia).

- Planejamento de Experimentos para avaliação de desempenho do processo de classificação e realização (inferência) da ontologia Pizza utilizando o processo de inicialização do módulo com a configuração padrão do reasoner Pellet (PE-Pizza).

- Planejamento de Experimentos para a avaliação de desempenho do módulo UDOnt-Q no processo de busca por Web Services com QoS (PE-Módulo).

No PE-Ontologia a variável de resposta analisada é o tempo de resposta para que a ontologia fique disponível para utilização. Essa variável é o tempo gasto para que o módulo leia, classifique, faça a inferência e carregue na memória do servidor a ontologia processada. Os experimentos desta avaliação foram replicados 10 vezes e o fator e os níveis considerados estão listados na Tabela 6.3.

Tabela 6.3: Fator e níveis relativos ao PE-Ontologia

\begin{tabular}{|c|c|c|}
\hline Fator & Níveis & Descrição \\
\hline Número de Serviços & 300, 600, 900 e 1200 & $\begin{array}{c}\text { Números de Serviços (indivíduos) cadastrados na } \\
\text { ontologia são classificados em Gold,Silver e Bronze. } \\
\text { Eles estão divididos igualmente entre os cinco prove- } \\
\text { dores também cadastrados na ontologia }\end{array}$ \\
\hline
\end{tabular}

O PE-Pizza é semelhante ao PE-Ontologia, porém trata-se de outra ontologia com quantidades de indivíduos diferentes. Os experimentos dessa avaliação também foram replicados 10 vezes e o fator e níveis considerados estão listados na Tabela 6.4 .

No PE-Módulo a variável de resposta analisada é o Tempo de Busca (TB) que considera o tempo gasto pelo módulo para encontrar o melhor serviço por meio da busca semântica na ontologia e incluir também o tempo para a consulta no registro UDDI recuperando a WSDL. Ou seja, 
Tabela 6.4: Fator e níveis relativos ao PE-Pizza

\begin{tabular}{|c|c|c|}
\hline Fator & Níveis & Descrição \\
\hline Número de Pizzas & $\mathbf{2 0 0 , 4 0 0 , 6 0 0 \text { e } 8 0 0}$ & $\begin{array}{c}\text { Números de Pizzas (indivíduos) cadastrados na } \\
\text { ontologia. Eles estão divididos igualmente entre } \\
\text { dois grupos, pizza com alta calorias (HighCalorie- } \\
\text { Pizza) e pizzas com baixas (LowCaloriePizza). }\end{array}$ \\
\hline
\end{tabular}

o TB é o tempo necessário para o módulo executar os passos do item 2 até o item 7 da Figura 5.8. Neste ponto, a ontologia já foi processada (classificada e inferida) e armazenada na memória durante a inicialização do servidor.

No PE-Modulo, os experimentos desta avaliação foram replicados 30 vezes e os fatores e os níveis considerados estão listados na Tabela 6.5.

Tabela 6.5: Fator e níveis relativos ao PE-Modulo

\begin{tabular}{|c|c|c|}
\hline Fator & Níveis & Descrição \\
\hline $\begin{array}{c}\text { Número de Serviços } \\
\text { (Fator A) }\end{array}$ & $\mathbf{3 0 0 , 6 0 0 \text { e } 1 2 0 0}$ & $\begin{array}{c}\text { Números de Serviços (indivíduos) cadastrados na } \\
\text { ontologia. Eles estão divididos igualmente } \\
\text { entre os cinco provedores cadastrados na ontolo- } \\
\text { gia e são classificados em Gold, Silver e Bronze. }\end{array}$ \\
\hline $\begin{array}{c}\text { Números de Clientes } \\
\text { (Fator B) }\end{array}$ & $\mathbf{1 5}$ e 30 & $\begin{array}{c}\text { Números de Clientes cadastrados na ontologia. } \\
\text { Também são classificados em Gold, Silver e Bronze. }\end{array}$ \\
\hline $\begin{array}{c}\text { Algoritmo de Busca } \\
\text { (Fator C) }\end{array}$ & $\begin{array}{c}\text { OntAlgorithmObject e } \\
\text { OntAlgorithmSPARQL }\end{array}$ & $\begin{array}{c}\text { Apenas os dois principais algoritmos de busca } \\
\text { com semântica serão avaliados. O algoritmo } \\
\text { OntAlgorithmRDQL não foi utilizado devido o } \\
\text { módulo utilizar a versão 2.6.3 do Jena, a qual } \\
\text { não possui suporte para a linguagem RDQL. }\end{array}$ \\
\hline
\end{tabular}

Além dos principais fatores destacados na Tabela 6.5, outros fatores foram fixados (Fatores Fixos) durante os experimentos do módulo (PE-Modulo). Esses fatores fixos e seus possíveis níveis estão listados na Tabela 6.6.

Os fatores da Tabela 6.6 são denominados fixos, pois durante cada experimento é utilizado (fixado) apenas um nível, não ocorrendo alteração de níveis no mesmo experimento. Dessa forma, estes fatores não serão considerados nos cálculos de influência de fatores (Seção 6.4.1).

A escolha de todos os fatores e níveis deve ser feita antes da inicialização do módulo, por meio do seu arquivo de configuração. Além disso, a configuração do ambiente pode sofrer algumas alterações de um experimento para outro, como por exemplo, a alteração do número de serviços cadastrados no registro UDDI (Fator A) e o número de threads que devem ser disparadas concorrentemente nas máquinas clientes (Fator B). Essa última configuração é necessária, pois os elementos de hardware disponíveis, listados na Tabela 6.1, não são suficientes para representar 15 e 30 clientes. Por esse motivo, foi preciso criar threads nas máquinas clientes para que representassem o número proposto de requisições concorrentes nos experimentos (cinco ou dez threads por cliente). 
Tabela 6.6: Fatores Fixos e possíveis níveis relativos ao PE-Modulo

\begin{tabular}{|c|c|c|}
\hline Fator & Níveis & Descrição \\
\hline $\begin{array}{c}\text { Número de Atributos de QoS } \\
\text { (AQ) }\end{array}$ & 3 e 2 & $\begin{array}{l}\text { Indica o número de atributos de QoS utilizados como } \\
\text { propriedades para a classificação no processo de equiva- } \\
\text { lência de classe durante a inferência da ontologia. Os } \\
\text { atributos são Tempo de Resposta, Disponibilidade e Custo. } \\
\text { No caso de apenas } 2 \text { atributos, o atributo Custo não foi } \\
\text { considerado. }\end{array}$ \\
\hline $\begin{array}{c}\text { Gravação de Log } \\
\text { (GL) }\end{array}$ & ON e OFF & Indica se o módulo gravará ou não arquivos de $\log s$. \\
\hline $\begin{array}{c}\text { Monitoração pelo Ganglia } \\
\text { (MG) }\end{array}$ & ON e OFF & $\begin{array}{l}\text { Indica se o módulo deverá ou não consultar } \\
\text { as informações do Ganglia em caso da busca retornar } \\
\text { mais de um serviço com a QoS adequada. }\end{array}$ \\
\hline $\begin{array}{c}\text { Monitoração de Carga } \\
\text { (MC) }\end{array}$ & ON e OFF & $\begin{array}{l}\text { Indica se a monitoração da carga de trabalho (CPU) } \\
\text { da máquina na qual o módulo executa estará ativa ou } \\
\text { não. Um script em Perl foi utilizado para armazenar } \\
\text { os valores de CPU a cada segundo. Para minimizar } \\
\text { a influência da carga gerada por eventuais rotinas } \\
\text { do Sistema Operacional foram considerados apenas } \\
\text { os valores superiores a 1\% de utilização de CPU. }\end{array}$ \\
\hline
\end{tabular}

A Figura 6.2 exibe a disposição dos elementos de hardware utilizados nos experimentos. As threads criadas consomem o serviço do UDOnt-Q para descobrir qual Web Service atende a sua exigência de QoS. Nos experimentos realizados, sempre haverá mais de um serviço que atende a essa exigência na ontologia ${ }^{17}$ e a opção de monitoramento poderá ou não estar ativa. Caso esteja, o módulo irá sempre consultar os resultados dos servidores a cada requisição.

\subsection{Avaliação de Desempenho dos Experimentos}

A avaliação de desempenho dos planejamentos PE-Ontologia e PE-Pizza possuem apenas um fator a ser considerado em cada um deles. O objetivo é identificar qual é o comportamento dos tempos gastos para os processos de realização (classificação e inferência), conforme o número de indivíduos aumenta na ontologia. Portanto, cada nível do fator corresponde a um experimento isolado. Dessa forma, foram realizados quatro experimentos para o PE-Ontologia e o PE-Pizza. Cada um foi repetido 10 (dez) vezes, obtendo-se um total de 40 (quarenta) testes para ambos. Os resultados obtidos e a análise do comportamento desses experimentos são apresentados na próxima seção.

Por outro lado, a avaliação de desempenho do planejamento PE-Módulo é mais complexa que as anteriores. Conforme mostra a Tabela 6.5, há três fatores (A, B e C) que variam em dois e três níveis. Além disso, há também quatro fatores que são fixos durante cada conjunto de experimento (CJE) do PE-Modulo. Um conjunto de experimento consiste em realizar experimentos com todas

\footnotetext{
${ }^{17}$ No Anexo 3 desta monografia é possível observar a composição e ordenação dos Web Services cadastrados na ontologia do UDOnt-Q.
} 


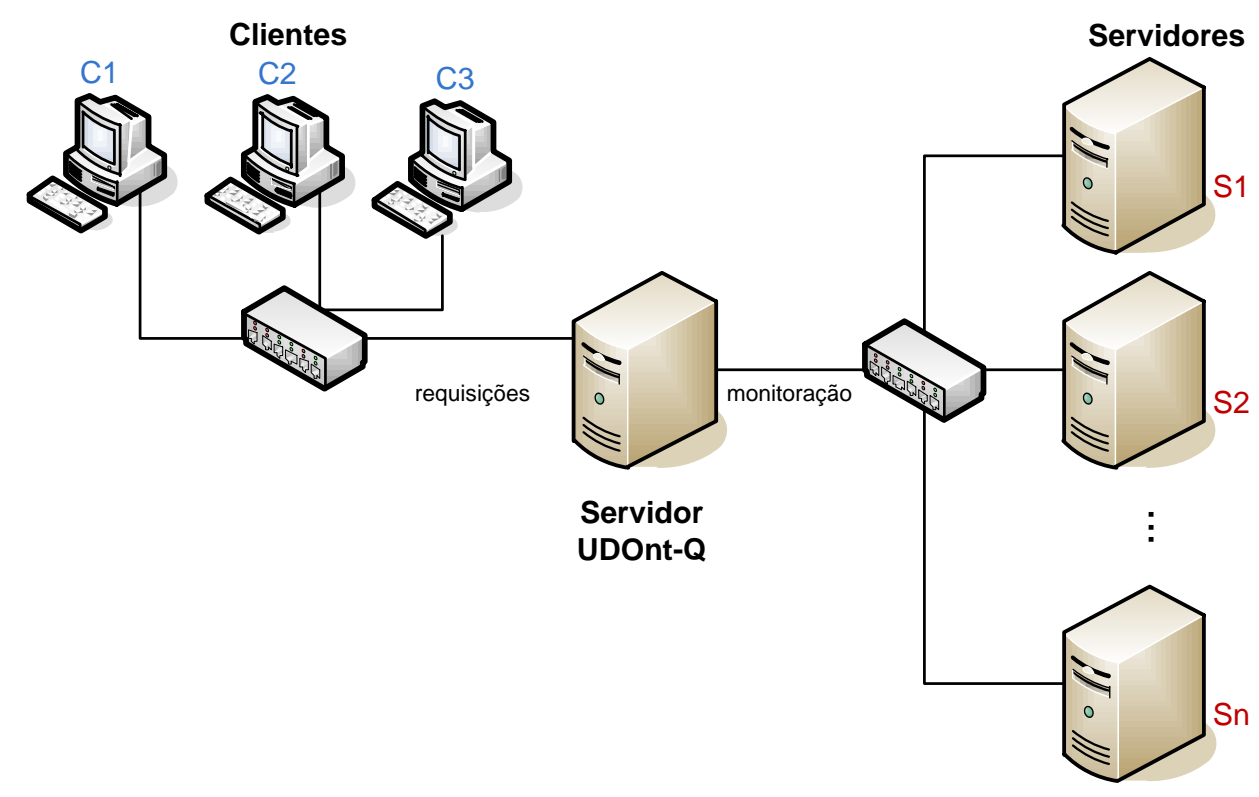

Figura 6.2: Visão resumida dos elementos de hardware utilizados.

as possibilidades dos fatores que variam, utilizando dois níveis para cada fator, e fixando uma opção de cada fator fixo.

Dessa forma, o PE-Modulo é composto por 8 conjuntos de experimentos (CJE), cada um deles composto por três fatores que variam em 2 (dois) níveis, e também por um conjunto específico de fatores (níveis) fixos. A escolha por dois níveis nos experimentos é justificada pelo fato da maioria dos fatores possuírem apenas dois níveis (com exceção do fator A). Essa escolha também visa possibilitar o cálculo do fatorial completo $2^{k}$ que limita que cada fator $(\mathrm{k})$ tenha no máximo dois níveis. O cálculo e análise da influência dos fatores (subseção 6.4.1) também são facilitados com o emprego do fatorial completo $2^{k}$. Cada variação de fator e nível corresponde a um novo experimento que deve ser analisado. Dessa forma, o projeto fatorial completo para cada conjunto de experimentos do módulo é de $2^{3}$ possíveis combinações.

Os oito conjuntos de experimentos (CJE) são baseados em dois projetos de fatorial completo $2^{k}$, denominados FC300-600 e FC300-1200 exibidos nas Tabelas 6.7 e 6.8 respectivamente. Os conjuntos de experimentos ímpares são baseados no FC300-600 e os pares no FC300-1200.

Esses dois projetos de fatorial completo (FC300-600 e FC300-1200) foram reproduzidos alterandose alguns fatores que permaneceram fixos durante os experimentos de cada conjunto. Foram utilizadas quatro combinações de fatores fixos totalizando os oito conjuntos de experimentos descritos na Tabela 6.9.

A variável de resposta relativa à carga de trabalho gerada pela utilização do módulo (consumo de CPU) é registrada apenas nos conjuntos de experimentos nos quais a monitoração de carga 
Tabela 6.7: Projeto Fatorial Completo para Avaliação de Desempenho do Módulo com 300 e 600

Serviços

\begin{tabular}{|c|c|c|c|}
\hline Experimento & A (N.Serviços) & B (N. Clientes) & C (Algoritmo) \\
\hline $\mathbf{1}$ & $\mathbf{3 0 0}$ & $\mathbf{1 5}$ & OntAlgorithmObject \\
\hline 2 & $\mathbf{3 0 0}$ & $\mathbf{1 5}$ & OntAlgorithmSPARQL \\
\hline 3 & $\mathbf{3 0 0}$ & $\mathbf{3 0}$ & OntAlgorithmObject \\
\hline 4 & $\mathbf{3 0 0}$ & $\mathbf{3 0}$ & OntAlgorithmSPARQL \\
\hline $\mathbf{5}$ & $\mathbf{6 0 0}$ & $\mathbf{1 5}$ & OntAlgorithmObject \\
\hline $\mathbf{6}$ & $\mathbf{6 0 0}$ & $\mathbf{1 5}$ & OntAlgorithmSPARQL \\
\hline 7 & $\mathbf{6 0 0}$ & $\mathbf{3 0}$ & OntAlgorithmObject \\
\hline $\mathbf{8}$ & $\mathbf{6 0 0}$ & $\mathbf{3 0}$ & OntAlgorithmSPARQL \\
\hline
\end{tabular}

Tabela 6.8: Projeto Fatorial Completo para Avaliação de Desempenho do Módulo com 300 e 1200 Serviços

\begin{tabular}{|c|c|c|c|}
\hline Experimento & A (N.Serviços) & B (N. Clientes) & C (Algoritmo) \\
\hline 1 & $\mathbf{3 0 0}$ & 15 & OntAlgorithmObject \\
\hline 2 & $\mathbf{3 0 0}$ & $\mathbf{1 5}$ & OntAlgorithmSPARQL \\
\hline 3 & $\mathbf{3 0 0}$ & $\mathbf{3 0}$ & OntAlgorithmObject \\
\hline 4 & $\mathbf{3 0 0}$ & $\mathbf{3 0}$ & OntAlgorithmSPARQL \\
\hline $\mathbf{5}$ & $\mathbf{1 2 0 0}$ & $\mathbf{1 5}$ & OntAlgorithmObject \\
\hline $\mathbf{6}$ & $\mathbf{1 2 0 0}$ & $\mathbf{1 5}$ & OntAlgorithmSPARQL \\
\hline 7 & $\mathbf{1 2 0 0}$ & $\mathbf{3 0}$ & OntAlgorithmObject \\
\hline $\mathbf{8}$ & $\mathbf{1 2 0 0}$ & $\mathbf{3 0}$ & OntAlgorithmSPARQL \\
\hline
\end{tabular}

Tabela 6.9: Conjuntos de Experimentos do PE-Modulo

\begin{tabular}{|c|c|c|c|c|c|}
\cline { 3 - 6 } N. Exp & $\begin{array}{c}\text { Conjunto de } \\
\text { Experimento }\end{array}$ & $\begin{array}{c}\text { Número de Atri- } \\
\text { butos de QoS (AQ) }\end{array}$ & $\begin{array}{c}\text { Gravação de } \\
\text { Log (GL) }\end{array}$ & $\begin{array}{c}\text { Monitoração } \\
\text { pelo Ganglia (MG) }\end{array}$ & $\begin{array}{c}\text { Monitoração } \\
\text { de Carga (MC) }\end{array}$ \\
\hline 1 & FC300-600 & 3 & ON & OFF & OFF \\
\hline 2 & FC300-1200 & 3 & ON & OFF & OFF \\
\hline 3 & FC300-600 & 2 & ON & OFF & OFF \\
\hline 4 & FC300-1200 & 2 & ON & OFF & OFF \\
\hline 5 & FC300-600 & 2 & ON & ON & ON \\
\hline 6 & FC300-1200 & 2 & ON & ON & ON \\
\hline 7 & FC300-600 & 2 & OFF & OFF & OFF \\
\hline 8 & FC300-1200 & 2 & OFF & OFF & OFF \\
\hline
\end{tabular}

(MC) estiver ativa (ON). Quando a gravação de $\log (\mathbf{G L})$ estiver desativada (OFF) não é possível registrar o tempo de busca (TB) do módulo, pois não são salvos registros de desempenho. Contudo, os clientes armazenam os tempos de respostas (RTC - Response Time in the Client) que podem ser avaliados. É importante recapitular que a ontologia sempre terá mais de um serviço que atendem às necessidades do cliente. Portanto, quando a monitoração pelo Ganglia (MG) estiver ativa (ON) o módulo deverá verificar qual é o provedor menos sobrecarregado naquele instante. 


\subsubsection{Cálculo da Influência dos Fatores}

No caso da avaliação de desempenho do módulo existem três fatores (A, B e C). Para se obter a influência de cada um desses fatores nas condições especificadas é preciso calcular a influência dos fatores no sistema avaliado. Para determinar a influência de cada fator, é possível empregar-se um modelo de regressão (Jain, 1991). O modelo para o projeto fatorial $2^{3}$ empregado nos experimentos deste trabalho, é apresentado na Equação (6.1).

$$
y=q_{0}+q_{A} x_{A}+q_{B} x_{B}+q_{C} x_{C}+q_{A B} x_{A B}+q_{B C} x_{B C}+q_{A C} x_{A C}+q_{A B C} x_{A B C}
$$

O próximo passo é substituir no modelo os valores $\left(y_{i}\right)$ das variáveis de resposta obtidos nos experimentos. Dessa forma, obtêm-se os valores de $q_{0}, q_{A}, q_{B}, q_{C}, q_{A B}, q_{B C}, q_{A C}$ e $q_{A B C}$. A Soma dos Quadrados Total (SST) desses valores $\left(q_{i}\right)$ fornece a variação total das variáveis de resposta dos experimentos e, consequentemente, as variações devidas à influência dos fatores e de suas interações. A equação da Soma dos Quadrados Total (SST) ou a Variação Total é dada pela Equação (6.2) (Jain, 1991):

$$
S S T=\sum_{i=1}^{2^{2}}\left(y_{i}-\bar{y}\right)^{2}
$$

Na Equação (6.2), a variável $\bar{y}$ é a média dos valores das variáveis de resposta obtidas em todas as replicações dos experimentos. Portanto, ao realizar a substituição de todos $2^{3}$ experimentos obtém-se a Equação (6.3):

$$
y=2^{3}\left(q_{A}^{2}+q_{B}^{2}+q_{C}^{2}+q_{A B}^{2}+q_{A C}^{2}+q_{B C}^{2}+q_{A B C}^{2}\right)
$$

A última etapa é determinar a influência de um determinado fator, para isso é preciso dividir a soma dos quadrados do fator pela soma dos quadrados total (Jain, 1991). Por exemplo, no caso do fator (A) o cálculo seria SSA/SST. Portanto, no experimento utilizado, a fórmula de SSA é apresentada na Equação (6.4):

$$
S S A=2^{3} q_{A}^{2}
$$


Os resultados obtidos na avaliação de desempenho e as influências dos fatores são apresentados na próxima seção.

\subsection{Análise dos Resultados}

Os planejamentos de experimentos apresentados na seção 6.3 foram realizados de acordo com a avaliação de desempenho da seção 6.4. Os resultados coletados durante os planejamentos das ontologias (PE-Ontologia e PE-Pizza) foram analisados e o comportamento resultante desses resultados é discutido na subseção 6.5.1. Os resultados do planejamento de experimento do módulo (PE-Modulo), a análise desses resultados e a influência dos fatores são apresentados na subseção 6.5.2.

\subsubsection{Análise dos Resultados das Ontologias}

Com relação à análise comportamental do processo de inferência das ontologias, podem-se observar nas Figuras 6.3 e 6.4 que para ambas o tempo gasto com esse processo aumentou conforme o número de indivíduos (Serviços ou Pizzas) cadastrados crescia. Para essa análise, fixou-se o número de clientes cadastrados na ontologia em 30.

Os resultados em gráficos em linhas, conforme exibem as Figuras 6.5 e 6.6, permitem uma visão mais clara da comparação dos resultados (Nakamura et al., 2011a).

Esses resultados mostram um avanço progressivo no aumento do tempo de resposta (TR) do módulo durante o processo de inferência utilizando-se três atributos de QoS para a equivalência de classe. Embora as ontologias não possam ser comparadas, pois há diferentes números de triplas RDF, classes, indivíduos, propriedades, etc., o comportamento para ambas não é linear. A Figura 6.7 faz uma comparação entre o comportamento do processo de inferência e de classificação da ontologia Pizza com um comportamento linear hipotético e exibe um aumento considerável quando a ontologia possui um maior número de indivíduos (Nakamura et al., 2011b).

Esse comportamento pode ser justificado pelo fato do racionalizador Pellet ocupar apenas um núcleo dos quatro disponíveis na CPU durante esse processo. Além disso, uma análise superficial no código fonte do racionalizador constatou a existência de diversas chamadas de métodos recursivos que exigem processamento e memória. Dessa forma, alguns dos recursos disponíveis não são utilizados em sua totalidade com eficiência enquanto outros podem chegar ao máximo de utilização (Nakamura et al., 2011b).

\subsubsection{Análise dos Resultados do Módulo UDOnt-Q}

Apesar do tempo de resposta para o processo de inferência ser longo, ele é executado apenas uma vez, durante a inicialização do módulo. Os resultados posteriores são referentes ao tempo 
TR Ontologia 30 Clientes \& 300,600,900,1200 Serviços

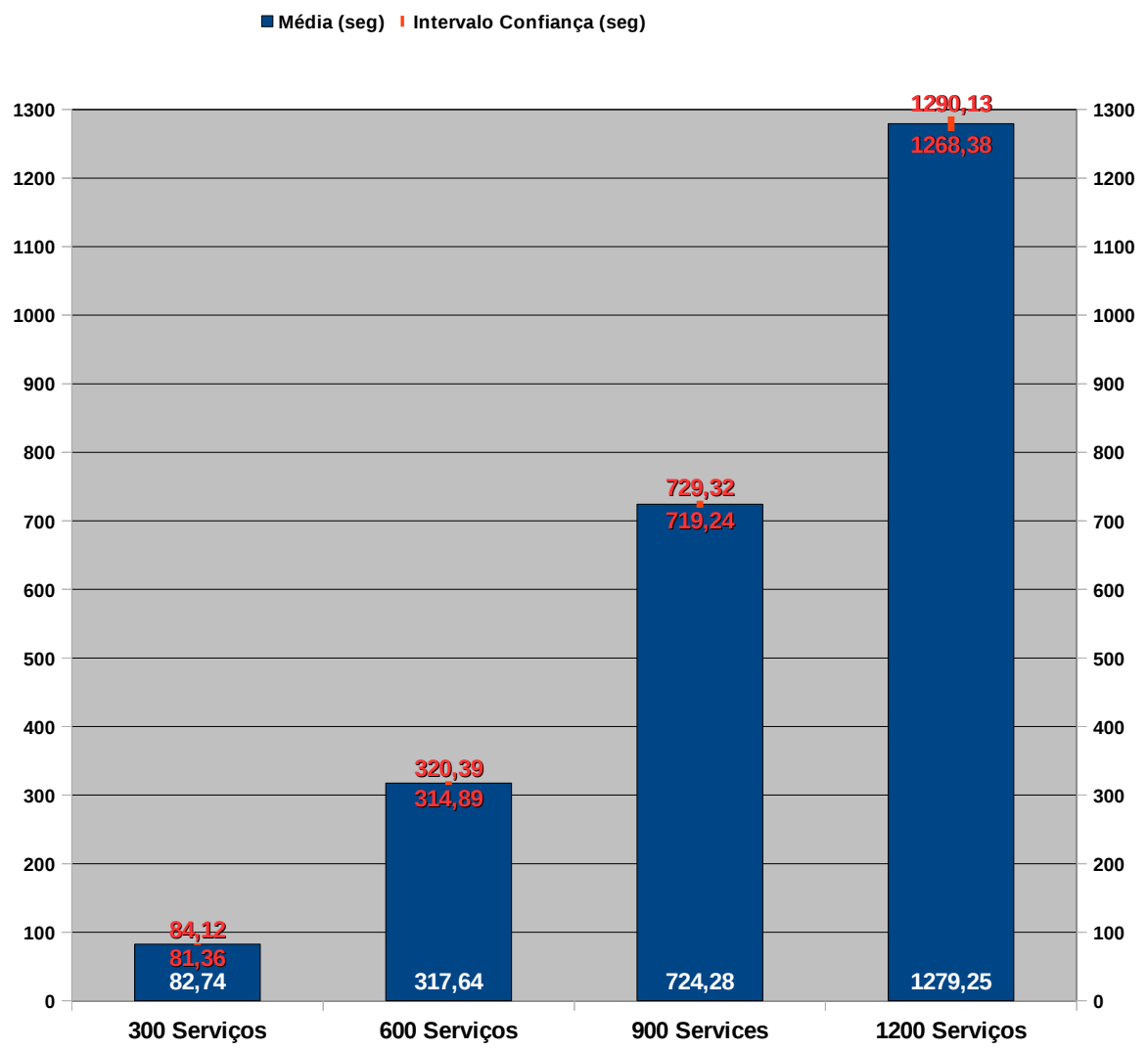

Figura 6.3: Comportamento do processamento de Inferência da Ontologia com 30 Clientes e 300, 600, 900 e 1200 Serviços (PE-Ontologia).

de busca (TB) do módulo por serviços com qualidade. Os resultados de cada conjunto de experimentos (Tabela 6.9) foram analisados e comparados. Devido à grande quantidade de resultados, percebeu-se a necessidade de omitir aqueles que são semelhantes de um experimento para outro com o objetivo de apresentar somente os resultados mais interessantes nesta seção. Contudo, os resultados podem ser encontrados na integra no Anexo 4 desta monografia. Portanto, essa medida evita a repetição de informações que não revelam novidades, principalmente para aqueles resultados que são estatisticamente iguais. Apenas alguns comentários, informando essa igualdade estatística dos resultados, serão mencionados nos momentos adequados.

As próximas duas primeiras subseções (6.5.2.1 e 6.5.2.2) apresentam os resultados dos conjuntos de experimentos 1 e 2 respectivamente. Nestes dois conjuntos de experimentos foram mantidos os mesmos níveis dos outros fatores com exceção dos níveis do fator Número de Serviços. Como eles são os primeiros conjuntos de experimentos apresentados nenhum resultado foi omitido e na subseção 6.5.2.2 são feitas algumas comparações entre eles.

Nas subseções 6.5.2.3 e 6.5.2.4 são discutidos os resultados dos conjuntos de experimentos 3 e 4 respectivamente. Esses experimentos são semelhantes aos experimentos 1 e 2 respectivamente. 
TR Pizza Ontologia - 200,400,600,800 Individuals

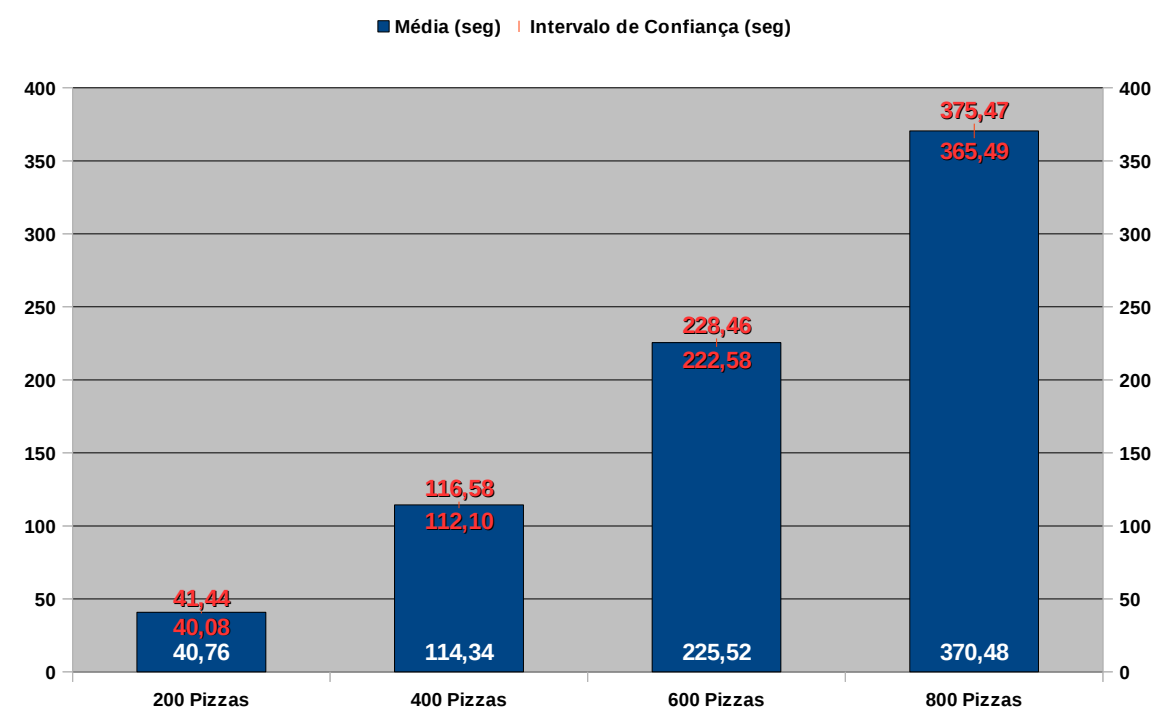

Figura 6.4: Comportamento do processamento de Inferência da Ontologia com 200,400, 600 e 800 Pizzas (PE-Pizza).

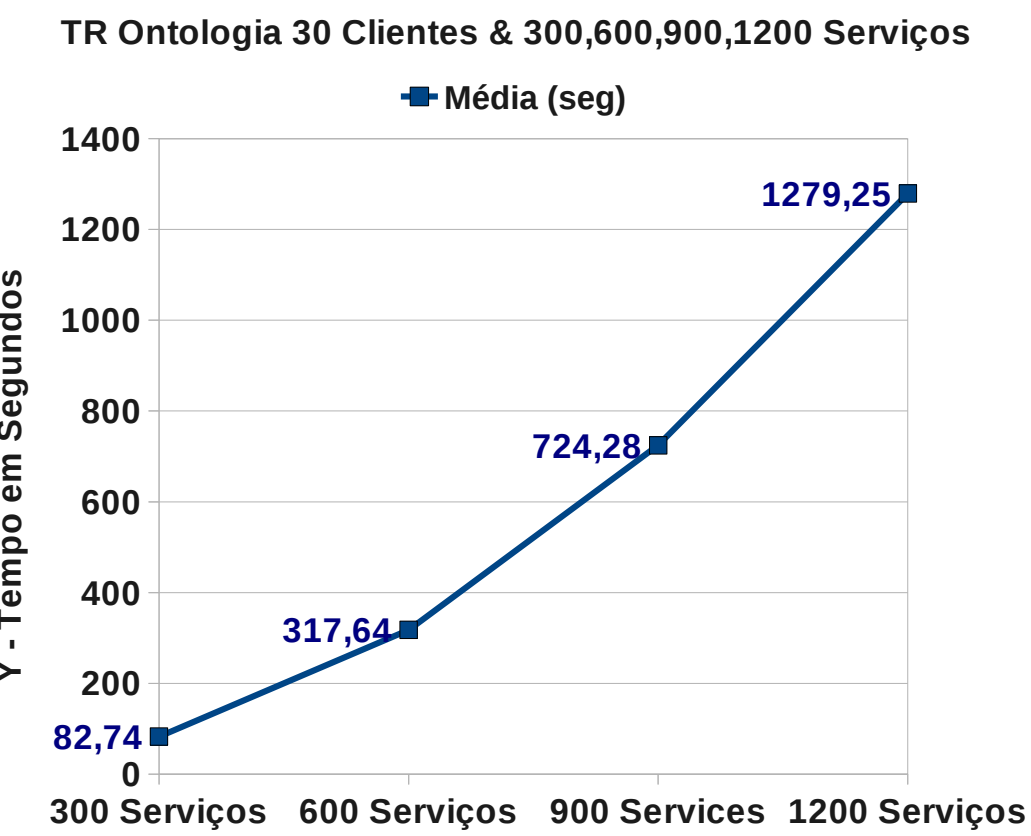

Figura 6.5: Comportamento do processamento de Inferência da Ontologia com 300, 600, 900 e 1200 Serviços (PE-Ontologia) (Nakamura et al., 2011b).

As subseções 6.5.2.5 e 6.5.2.6 apresentam resultados com a função de monitoramento por parte do monitor Ganglia ativada. Nestes experimentos 5 e 6 são mantidos todos os fatores e níveis dos experimentos 1 e 2, com a diferença de que nos experimentos 5 e 6 todos os fatores fixos estão ativados (Gravação de Log, Monitoração de Carga e Monitoração de QoS pelo Ganglia). 
TR Pizza Ontologia - 200,400,600,800 Individuals

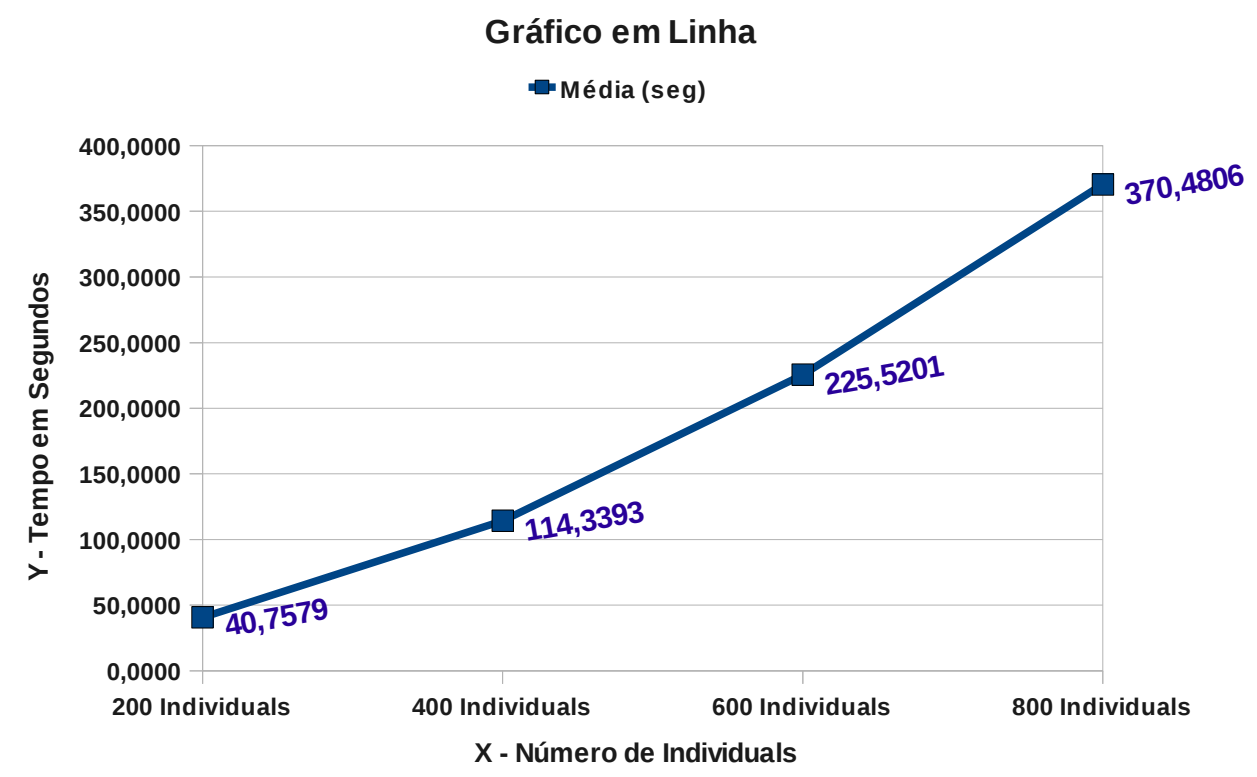

Figura 6.6: Comportamento do processamento de Inferência da Ontologia com 200, 400, 600 e 800 Pizzas (PE-Pizza) (Nakamura et al., 2011b).

TR Pizza Ontologia - 200,400,600,800 Individuals

Gráfico em Linha

$\square$ Média (seg) $\leadsto$ Linear Hipotético(seg)

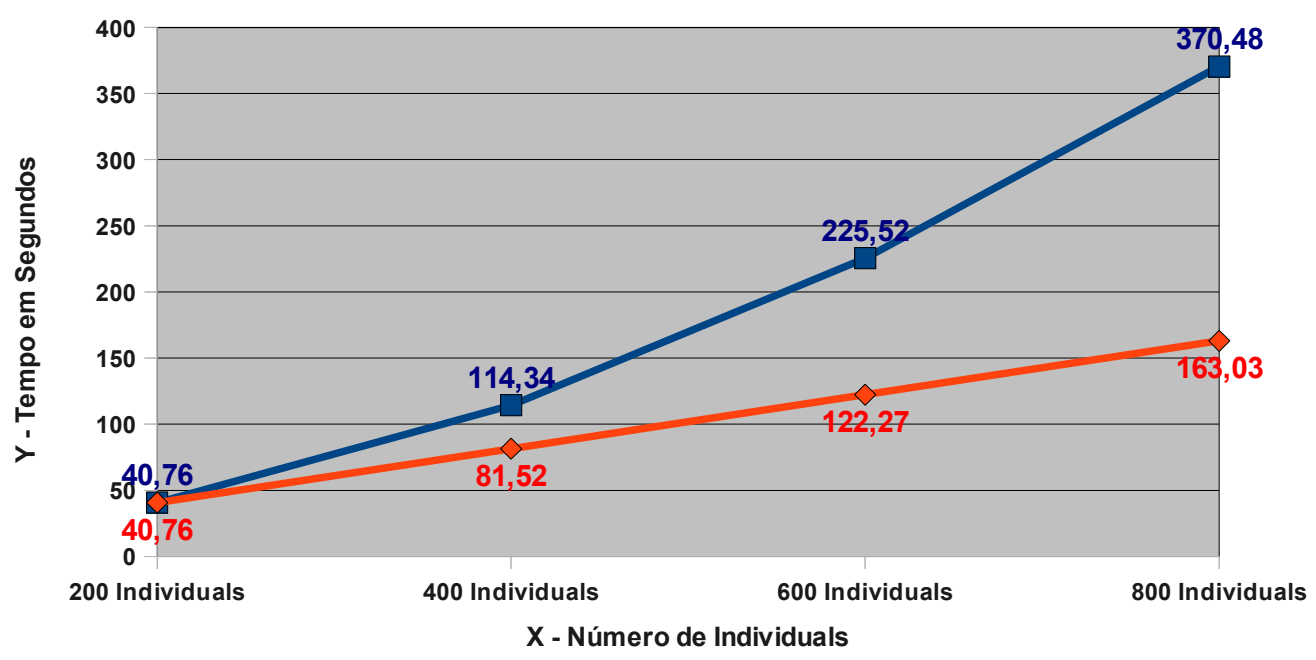

Figura 6.7: Gráfico em Linha de comparação do Comportamento do processamento de Inferência com 200, 400, 600 e 800 Pizzas (PE-Pizza) com um Comportamento Linear Hipotético (Nakamura et al., 2011b).

As subseções 6.5.2.7 e 6.5.2.8 contêm resultados dos conjuntos de experimentos 7 e 8 que mantêm todos os fatores e níveis dos experimentos 5 e 6 respectivamente. Porém, os fatores fixos foram desativados (Gravação de Log, Monitoração de Carga e Monitoração de QoS pelo Ganglia). Dessa forma, é possível verificar e comparar alguns resultados entre os conjuntos de experimentos 
com os recursos do módulo ativados e desativados. Contudo, o fato da gravação do log estar desativada permite apenas comparar os tempos de resposta do cliente, pois o módulo deixa de registrar qualquer informação de tempo ou desempenho no arquivo de log e na base de dados.

\subsubsection{Conjunto de Experimentos 1}

Neste conjunto de experimentos é possível analisar os resultados dos experimentos obtidos com 300 e 600 serviços (Fator A), 15 e 30 clientes (Fator B) e ambos os algoritmos (Fator C). Ainda neste conjunto de experimentos são considerados os fatores fixos de acordo com a Tabela 6.9 .

A Figura 6.8 exibe a variação no tempo de busca (TB) quando o número de serviços é duplicado, passando de 300 para 600 serviços. Utilizando o algoritmo OntAlgorithmObject é possível observar que um aumento de $100 \%$ no número de serviços ocasiona em um aumento por volta de $50 \%$ no tempo de busca. Com o algoritmo OntAlgorithmSPARQL essa diferença é muito maior. Ainda na Figura 6.8 observa-se uma grande variação do tempo de busca (TB) quando o algoritmo OntAlgorithmObject é substituído pelo algoritmo OntAlgorithmSPARQL.

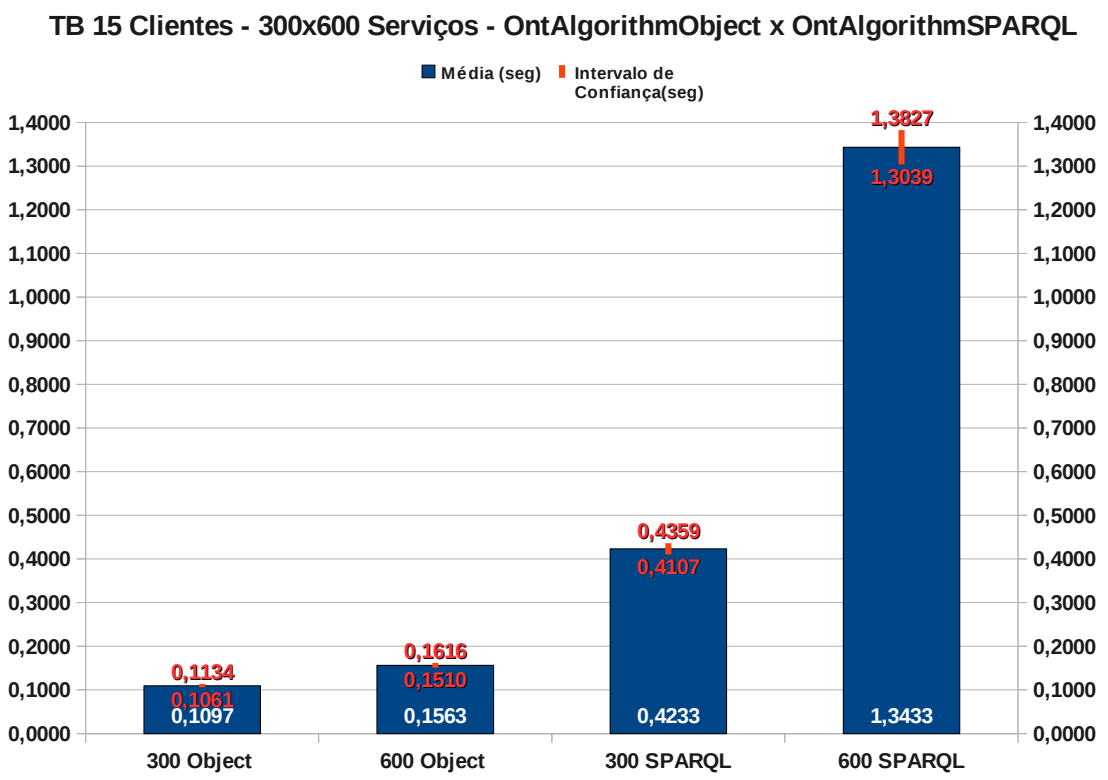

Figura 6.8: Tempo de Busca (TB) utilizando os algoritmos com 15 clientes (CJE-1).

O algoritmo OntAlgorithmSPARQL leva um tempo muito maior que o algoritmo OntAlgorithmObject para encontrar o melhor serviço para o cliente. Uma das razões para essa diferença de desempenho é que o OntAlgorithmObject teve seu desenvolvimento baseado especificamente para a ontologia do UDOnt-Q, enquanto o OntAlgorithmSPARQL exige pacotes adicionais para o seu funcionamento os quais permitem uma certa flexibilidade em alterações na estrutura da ontologia (uma alteração exige apenas a reformulação da query de consulta).

Uma variação um pouco maior no tempo de busca (TB) é exibida na Figura 6.9, ainda utilizando 300 e 600 serviços com ambos os algoritmos. Porém, para obter esses resultados foram feitas 30 
requisições de clientes concorrentes. Observam-se que os tempos de busca com 30 clientes são maiores do que com 15 clientes. Esse resultado já era esperado, pois quanto maior o número de requisições de clientes maior é a carga de trabalho imposta ao módulo.

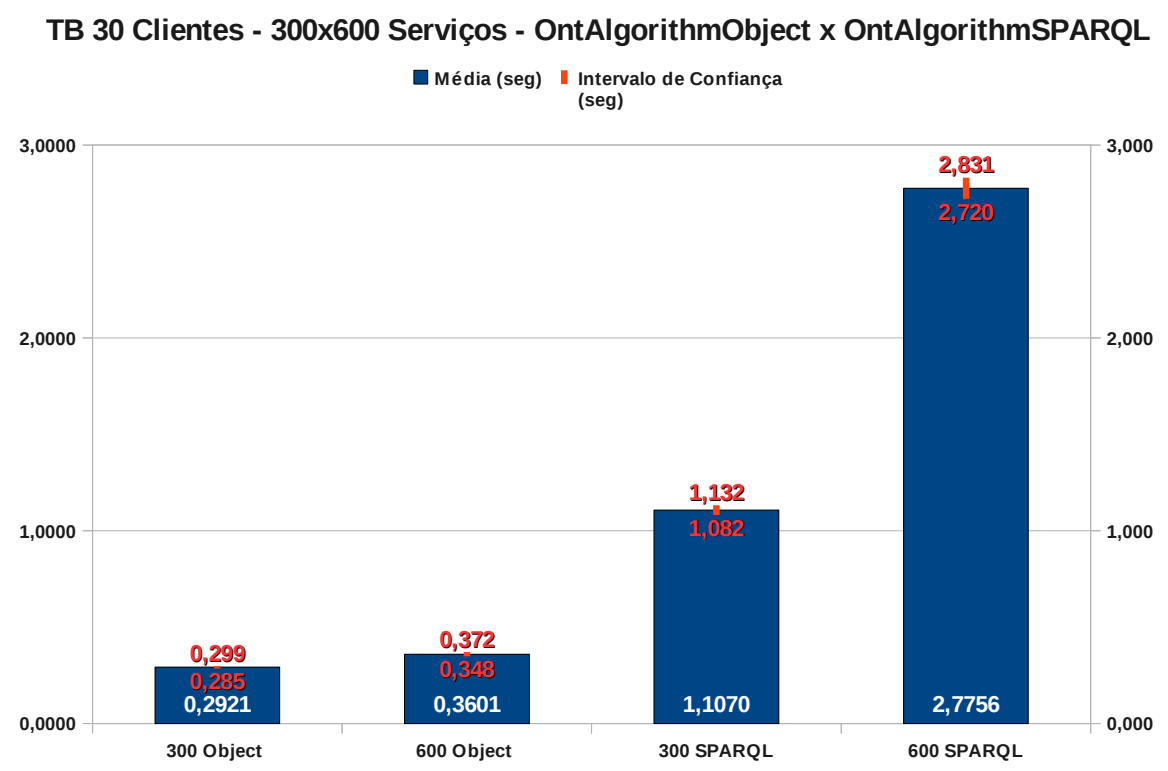

Figura 6.9: Tempo de Busca (TB) utilizando os algoritmos com 30 clientes (CJE-1).

A Figura 6.10 exibe os tempos de resposta para os 15 clientes (RTC) obterem a resposta do módulo com 300 e 600 serviços utilizando ambos os algoritmos.

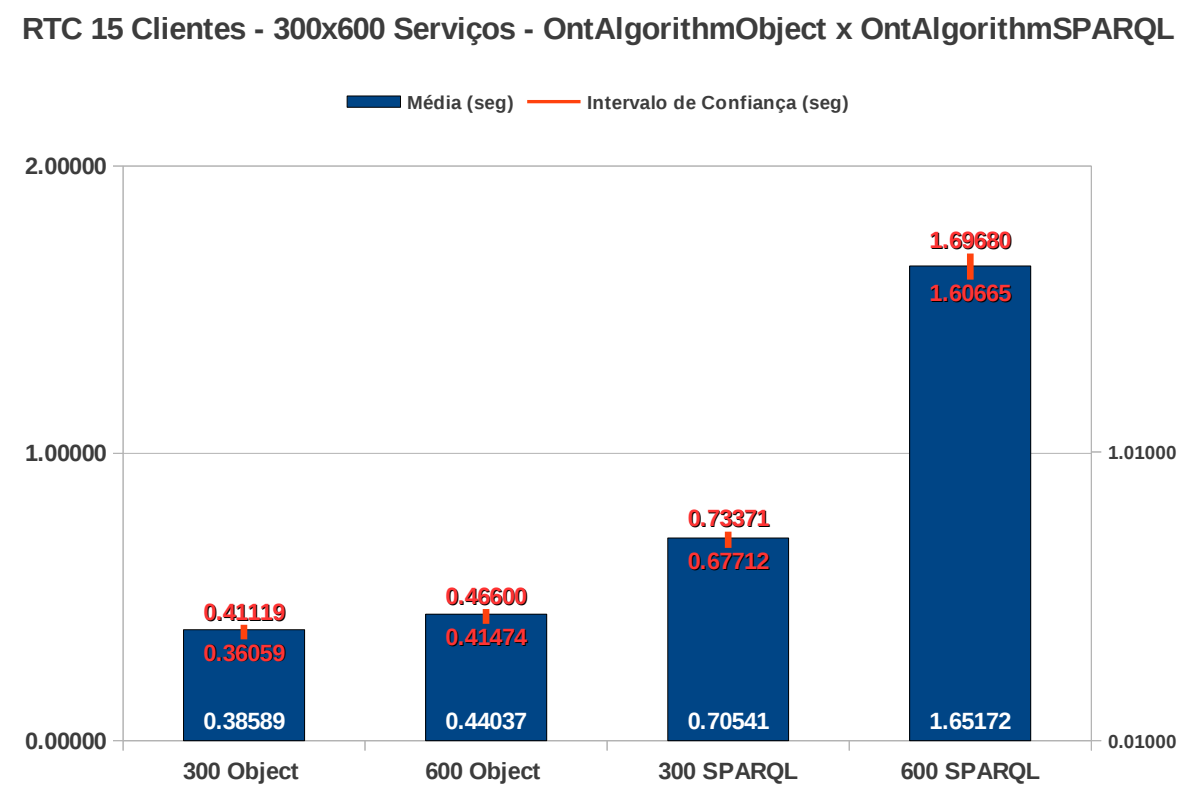

Figura 6.10: Tempo de Resposta para o Cliente (RTC) utilizando os algoritmos com 15 clientes (CJE-1).

O RTC é o tempo contabilizado desde o início da requisição até a chegada da resposta no cliente (desde a etapa 1 até a 8 da Figura 5.8). Portanto, esse tempo inclui o tempo de busca (TB) da Figura 
6.8, o tempo de processamento para o empacotamento e desempacotamento da mensagem SOAP além do tempo gasto no tráfego dos dados na rede. Observa-se que a diferença nos tempos de 300 e 600 serviços é minimizada devido à inclusão dos tempos citados anteriormente, mas o resultado com 300 serviços ainda é estatisticamente menor do que o resultado com 600 serviços.

A Figura 6.11 exibe os tempos de resposta para os 30 clientes (RTC) obterem uma resposta do módulo utilizando os algoritmos OntAlgorithmObject e OntAlgorithmSPARQL com 300 e 600 serviços. Em concordância aos resultados da Figura 6.9, o desempenho do OntAlgorithmObject permaneceu melhor que o do OntAlgorithmSPARQL, mesmo envolvendo outros tempos (processamento SOAP e transmissão na rede) neste resultado.

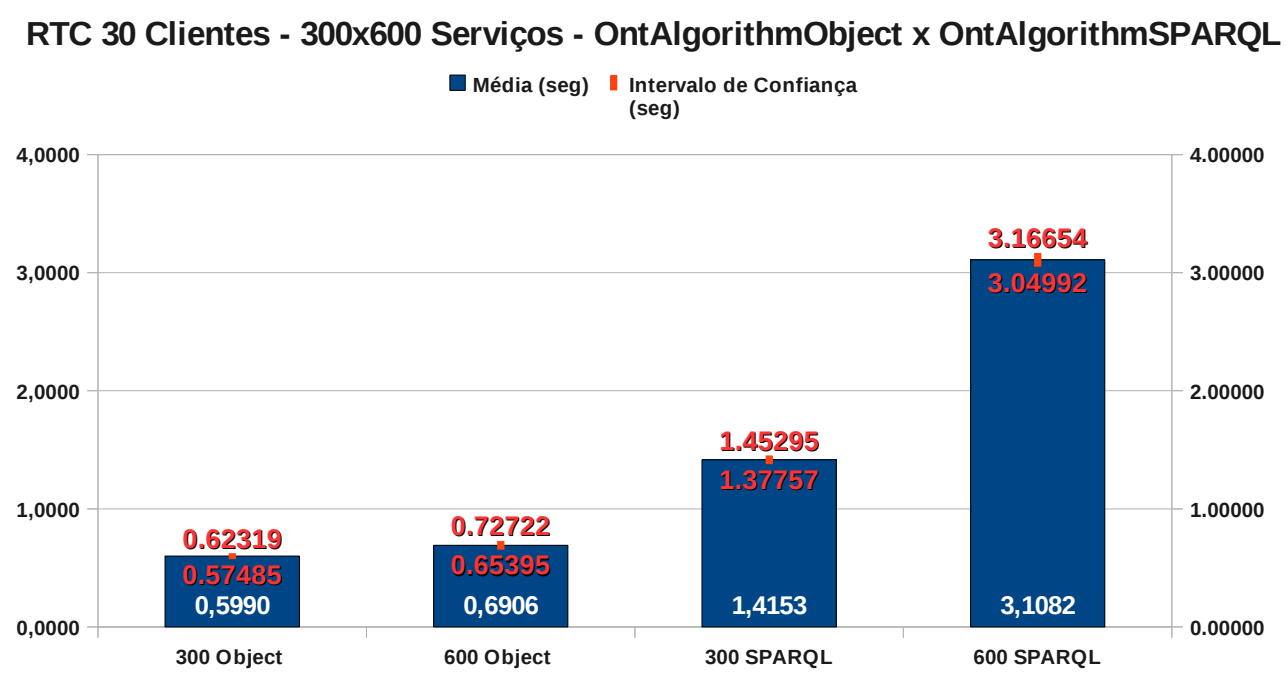

Figura 6.11: Tempo de Resposta para o Cliente (RTC) utilizando os algoritmos com 30 clientes (CJE-1).

As médias dos tempos de resposta para os clientes (RTC) foram utilizadas para calcular a vazão (Throughput) do módulo. A Tabela 6.10 lista as possibilidades do fatorial completo $2^{3}$ com as médias dos tempos de busca (TB) do módulo, as médias dos tempos de resposta para os clientes (RTC) e a partir dessas médias são calculadas as vazões. A Figura 6.12 exibe as porcentagens de influência de cada fator durante a execução dos experimentos na busca por serviços com qualidade.

Tabela 6.10: Fatorial completo $2^{3}$ e Médias do Tempo de Busca (TB) do CJE-1

\begin{tabular}{|c|c|c|c|c|c|c|}
\hline Experimento & $\begin{array}{c}\text { Fator A } \\
\text { (N. Serviços) }\end{array}$ & $\begin{array}{c}\text { Fator B } \\
\text { (N. Clientes) }\end{array}$ & $\begin{array}{c}\text { Fator C } \\
\text { (Algoritmo) }\end{array}$ & TB Média & RTC Média & Throughput \\
\hline 1 & 300 & 15 & Object & 0,10972 & 0,38589 & 2,59 \\
\hline 2 & 300 & 15 & SPARQL & 0,42332 & 0,70541 & 1,42 \\
\hline 3 & 300 & 30 & Object & 0,29207 & 0,59902 & 1,67 \\
\hline 4 & 300 & 30 & SPARQL & 1,10697 & 1,41526 & 0,71 \\
\hline 5 & 600 & 15 & Object & 0,15632 & 0,44037 & 2,27 \\
\hline 6 & 600 & 15 & SPARQL & 1,34331 & 1,65172 & 0,61 \\
\hline 7 & 600 & 30 & Object & 0,36009 & 0,69058 & 1,45 \\
\hline 8 & 600 & 30 & SPARQL & 2,77555 & 3,10823 & 0,32 \\
\hline
\end{tabular}




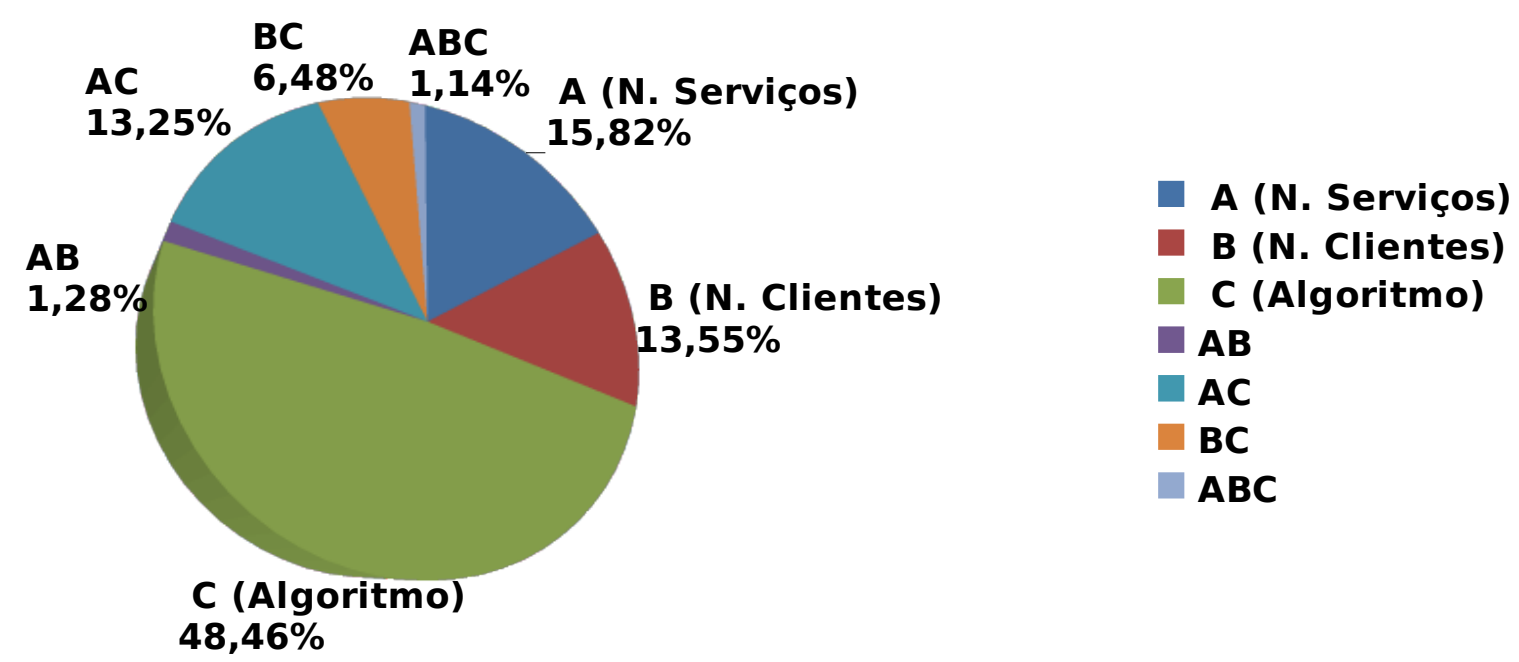

Figura 6.12: Porcentagem de Influência de cada fator no tempo de busca (TB) do módulo durante os experimentos (CJE-1).

Analisando os resultados da Tabela 6.10 e as porcentagens de influência de cada fator na Figura 6.12 é possível notar que o fator C (Algoritmo) com 48,46\% é o que exerce maior influência nos experimentos. Esse resultado era previsto, pois é grande a discrepância dos tempos de resposta de um algoritmo para outro. O segundo fator que mais influenciou foi o fator A (Número de Serviços) com 15,82\%, seguido pelo fator B (Número de Clientes) com 13,55\%. Esses dois últimos fatores exercem uma influência considerável no sistema e podem aumentar conforme o número de serviços e clientes aumenta. No Conjunto de Experimentos 2, descrito na próxima seção, será possível observar a influência do fator A com um número maior de serviços. Na quarta e quinta posições ficaram as combinações de fatores $\mathrm{AC}$ com $13,25 \%$ e BC com 6,48\% respectivamente, observandose que as combinações que envolveram o fator $\mathrm{C}$ apresentaram maior influência, com exceção da combinação dos fatores $\mathrm{ABC}$ com 1,14\%. A combinação $\mathrm{AB}$ ficou com 1,28\% de influência nos experimentos.

Neste conjunto de experimentos a Monitoração de Carga (MC) estava inativa (OFF) e por esse motivo não há resultados sobre a carga de processamento do módulo.

\subsubsection{Conjunto de Experimentos 2}

O segundo conjunto de experimentos é semelhante ao primeiro, com a diferença de que o fator A tem 300 e 1200 serviços ao invés de 300 e 600 serviços. Portanto, esse experimento utiliza o fatorial completo da Tabela 6.8 e os mesmos fatores fixos do primeiro conjunto de experimentos conforme descrito na Tabela 6.9 .

Seguindo a mesma sequencia do primeiro conjunto de experimentos, a Figura 6.13 exibe a variação no tempo de busca (TB) quando o número de serviços é quadruplicado, passando de 300 para 1200 serviços. Utilizando o algoritmo OntAlgorithmObject é possível observar que um 
aumento de 300 para 1200 serviços ocasiona em um aumento de aproximadamente $150 \%$ no tempo de busca. Nota-se que o algoritmo OntAlgorithmObject manteve seu desempenho muito melhor que o algoritmo OntAlgorithmSPARQL. Há uma grande diferença no intervalo de tempo entre as busca feitas com os algoritmos, a justificativa é a mesma dada no conjunto de experimentos 1 (CJE-1). Porém, o problema se agrava quando o número de serviços é maior, como é o caso dos resultados com 1200 serviços. Para que a exibição do gráfico não ficasse desproporcional, utilizou-se a escala logarítmica.

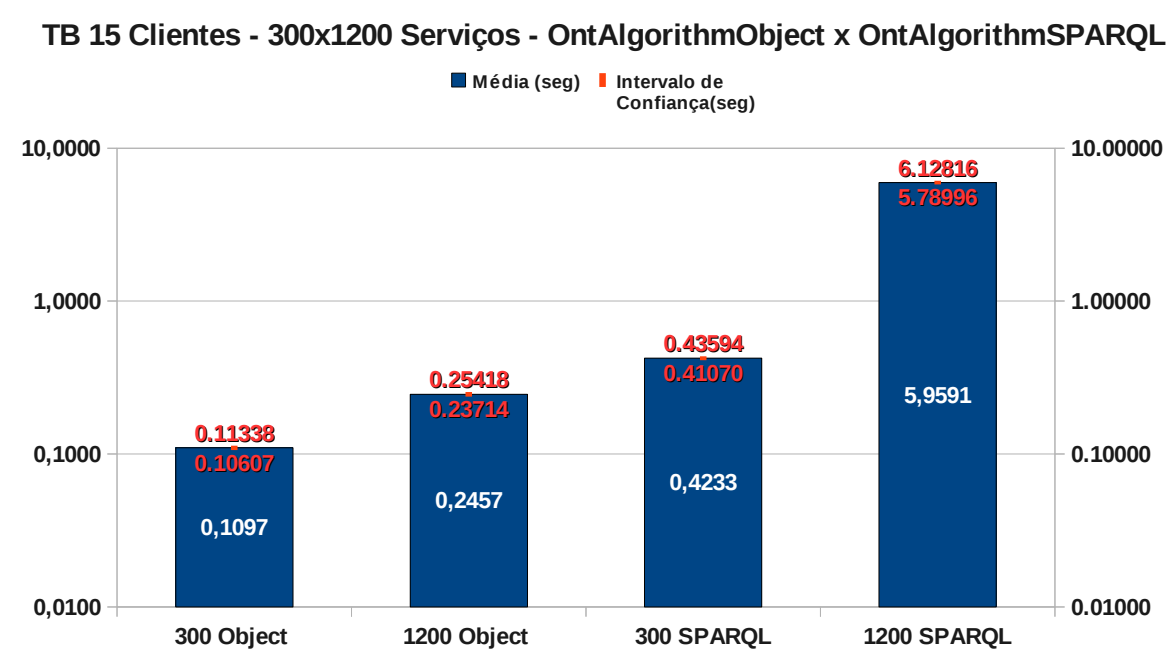

Figura 6.13: Tempo de Busca (TB) utilizando os algoritmos com 15 clientes (CJE-2).

A Figura 6.14, também em escala logarítmica, exibe um aumento no tempo de busca (TB) quando o número de clientes concorrentes é duplicado. Correspondente aos resultados da Figura 6.9, esse aumento é devido ao enfileiramento das requisições, pois o módulo atende uma requisição por vez.

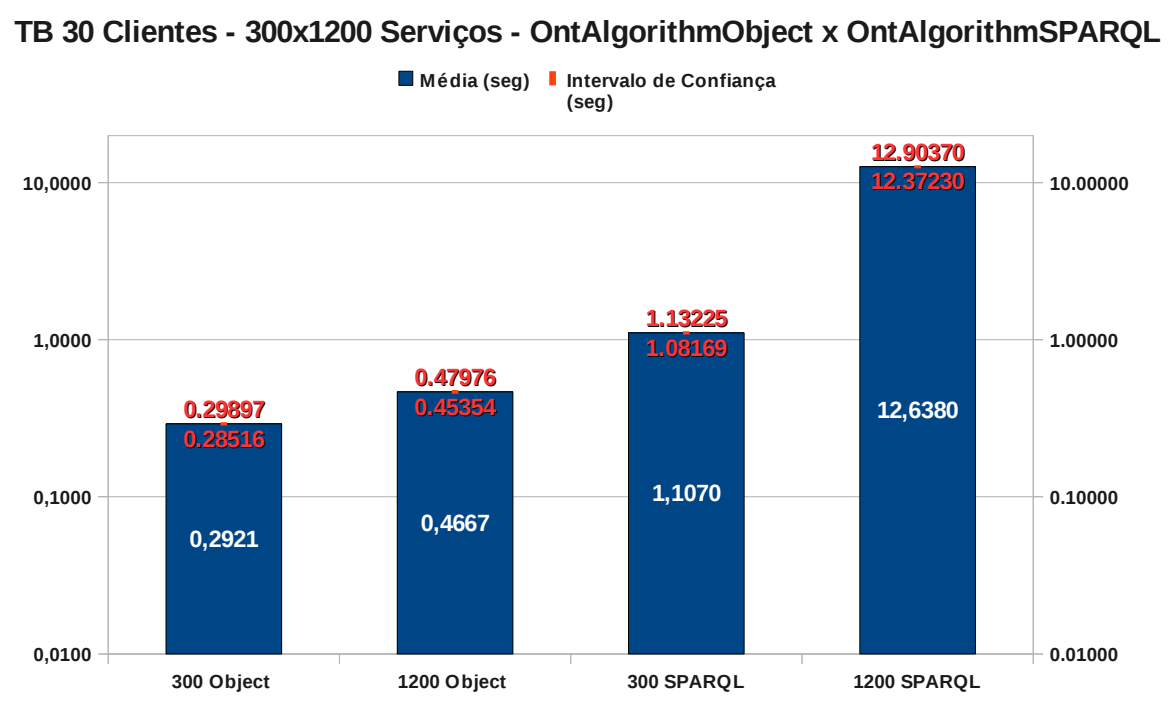

Figura 6.14: Tempo de Busca (TB) utilizando os algoritmos com 30 clientes (CJE-2). 
Com relação ao tempo de resposta para o cliente (RTC), observa-se um comportamento similar ao do conjunto de experimentos 1 (CJE-1). A Figura 6.15 mostra esse tempo com 300 e 1200 serviços utilizando ambos os algoritmos para atender a 15 requisições de clientes correntes. Assim como no conjunto de experimentos 1 , a diferença do tempo de resposta para o cliente entre as quantidades de serviços é minimizada pelo fato deste tempo considerar outros tempos como, por exemplo, o tempo de processamento das mensagens SOAP.

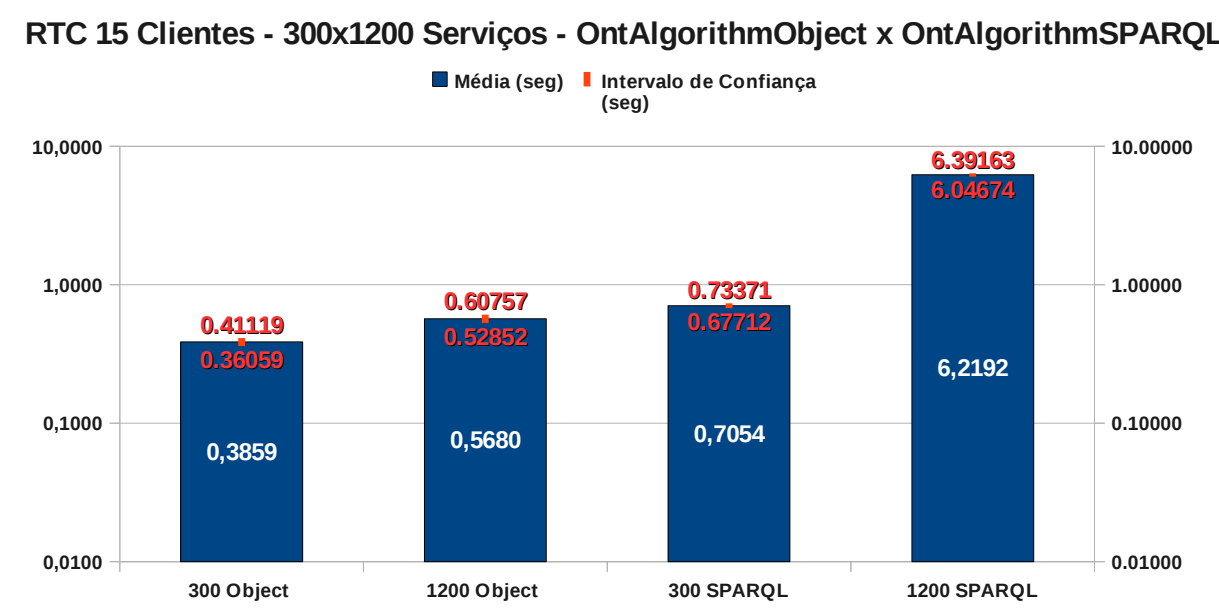

Figura 6.15: Tempo de Resposta para o Cliente (RTC) utilizando os algoritmos com 15 clientes (CJE-2).

Os resultados da Figura 6.16, apresentados com escala logarítmica, exibem um aumento no tempo de busca (TB) quando o número de requisições dos clientes é dobrado, passando de 15 clientes para 30 clientes. Esses resultados eram esperados, pois o mesmo efeito aconteceu quando o tempo de busca aumentou devido à duplicação do número de requisições.

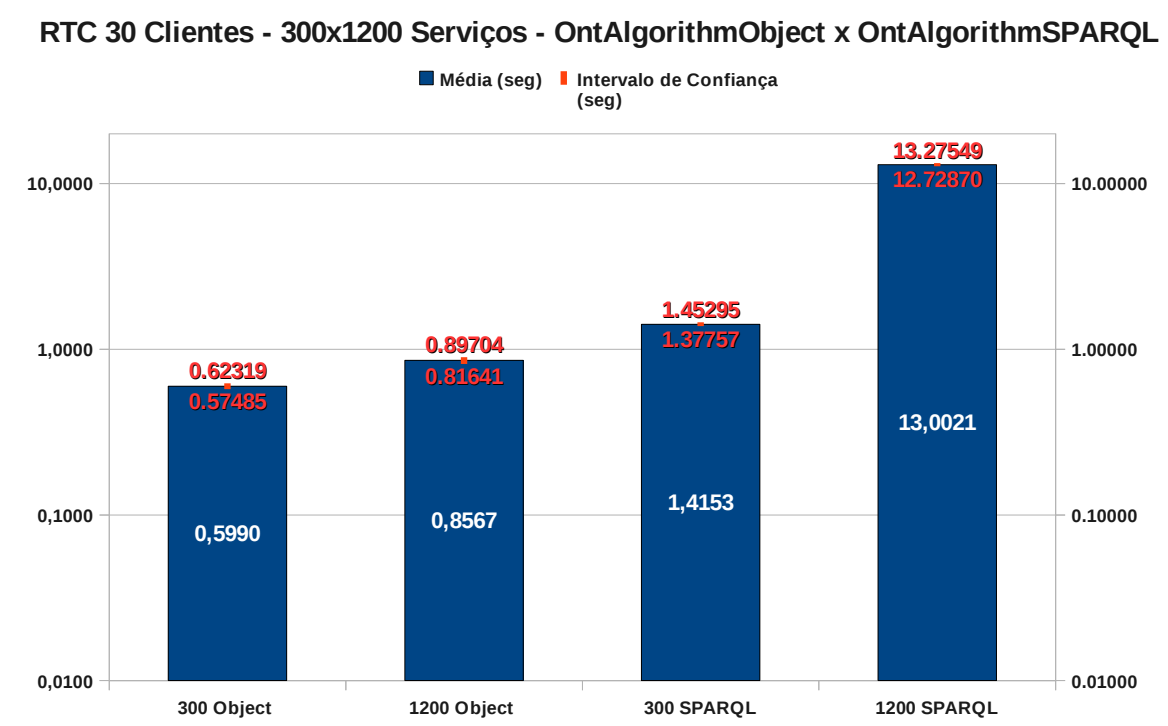

Figura 6.16: Tempo de Resposta para o Cliente (RTC) utilizando os algoritmos com 30 clientes (CJE-2). 
Reunindo alguns resultados do primeiro conjunto de experimentos com os resultados deste segundo é possível realizar uma comparação entre os tempos de busca com os três níveis do Fator A (Número de Serviços: 300, 600 e 1200). Essa união está ilustrada na Figura 6.17 que exibe os resultados dos tempos de busca (TB) para 30 clientes concorrentes.

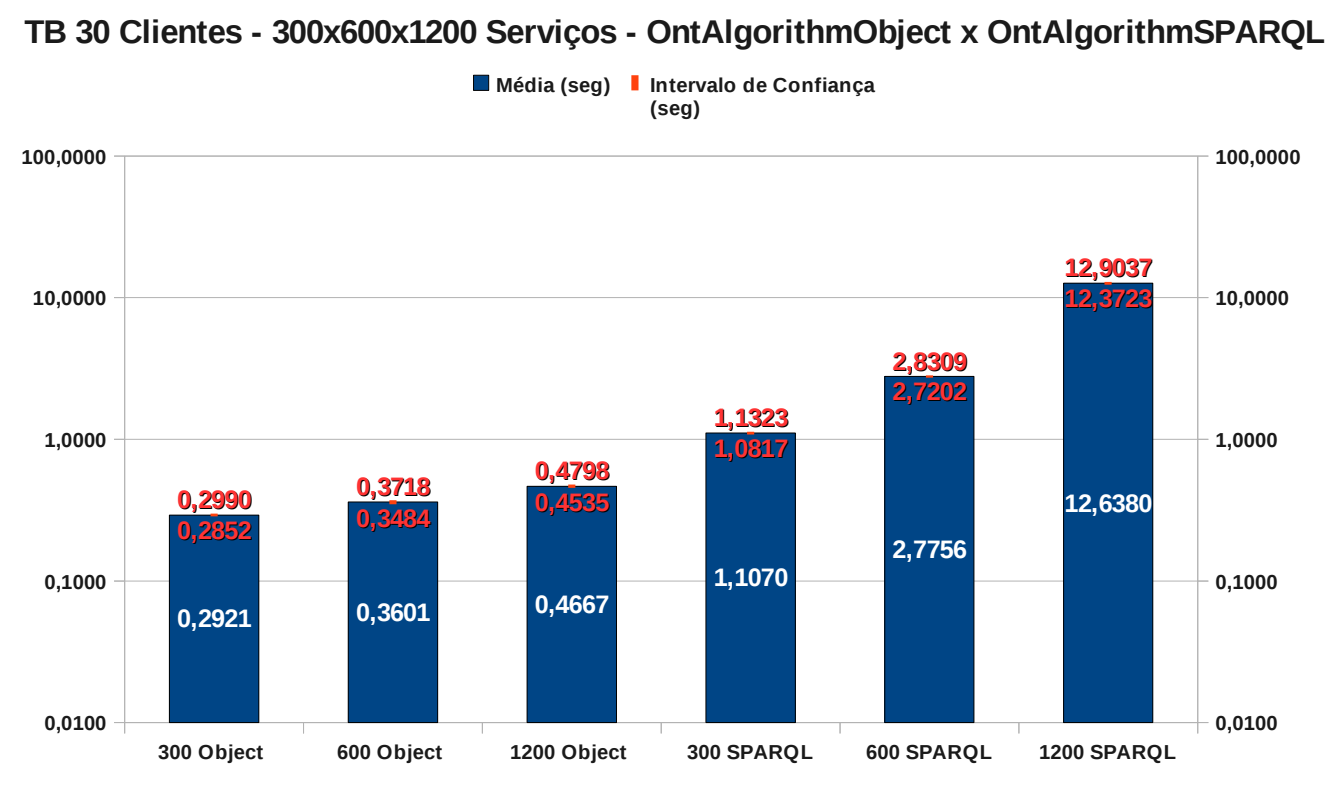

Figura 6.17: Tempo de Busca (TB) utilizando os algoritmos com 30 clientes e todos os serviços (CJE-1 e 2).

Analisando os resultados da Figura 6.17 fica clara a vantagem do algoritmo OntAlgorithmObject sobre o algoritmo OntAlgorithmSPARQL. Os resultados mostram que o primeiro leva menos tempo para localizar/selecionar os serviços desejados, mesmo precisando procurar em um número maior de serviços cadastrados. Ou seja, o algoritmo OntAlgorithmObject é mais rápido buscando em 1200 serviços do que o algoritmo OntAlgorithmSPARQL buscando em apenas 300 serviços.

A Tabela 6.11 exibe as possibilidades do fatorial completo $2^{3}$ com as médias dos tempos de busca (TB) do módulo, as médias dos tempos de resposta para os clientes (RTC) e a partir dessas médias são calculadas as vazões (Throughput). Na Figura 6.18 são exibidas as porcentagens de influência de cada fator durante a execução dos experimentos na busca por serviços com qualidade.

Os resultados da Tabela 6.11 em conjunto com as porcentagens de influência de cada fator na Figura 6.18 exibem o fator C (Algoritmo), que com 32,14\% de influência, é o fator que exerce maior influência nos experimentos. Esse resultado era esperado, pois da mesma forma que ocorreu no CJE-1 foi grande a discrepância dos tempos de resposta entre um algoritmo e outro. O segundo fator que mais influenciou foi o fator A (Número de Serviços) com 26,84\% ficando a frente da combinação dos fatores AC com quase 24,96\%. Esses resultados indicam que, nos níveis e fatores escolhidos para a análise, os fatores $\mathrm{C}$ e A são os que exercem influência considerável no sistema. O Fator B (Número de Clientes) ficou com apenas 5,36\% de influência, embora essa influência não seja tão representativa quando comparada aos outros fatores ( $\mathrm{C}$ e A). O fator B com níveis maiores, como por exemplo, 15 e 150 clientes (10 vezes mais) provavelmente terá uma influência maior no 
Tabela 6.11: Fatorial completo $2^{3}$ e Médias do Tempo de Busca (TB) do CJE-2

\begin{tabular}{|c|c|c|c|c|c|c|}
\hline Experimento & $\begin{array}{c}\text { Fator A } \\
\text { (N. Serviços) }\end{array}$ & $\begin{array}{c}\text { Fator B } \\
\text { (N. Clientes) }\end{array}$ & $\begin{array}{c}\text { Fator C } \\
\text { (Algoritmo) }\end{array}$ & TB Média & RTC Média & Throughput \\
\hline 1 & 300 & 15 & Object & 0,10972 & 0,38589 & 2,59 \\
\hline 2 & 300 & 15 & SPARQL & 0,42332 & 0,70541 & 1,42 \\
\hline 3 & 300 & 30 & Object & 0,29207 & 0,59902 & 1,67 \\
\hline 4 & 300 & 30 & SPARQL & 1,10697 & 1,41526 & 0,71 \\
\hline 5 & 1200 & 15 & Object & 0,24566 & 0,568 & 1,76 \\
\hline 6 & 1200 & 15 & SPARQL & 5,95906 & 6,2192 & 0,16 \\
\hline 7 & 1200 & 30 & Object & 0,46665 & 0,8567 & 1,17 \\
\hline 8 & 1200 & 30 & SPARQL & 12,638 & 13,0021 & 0,08 \\
\hline
\end{tabular}

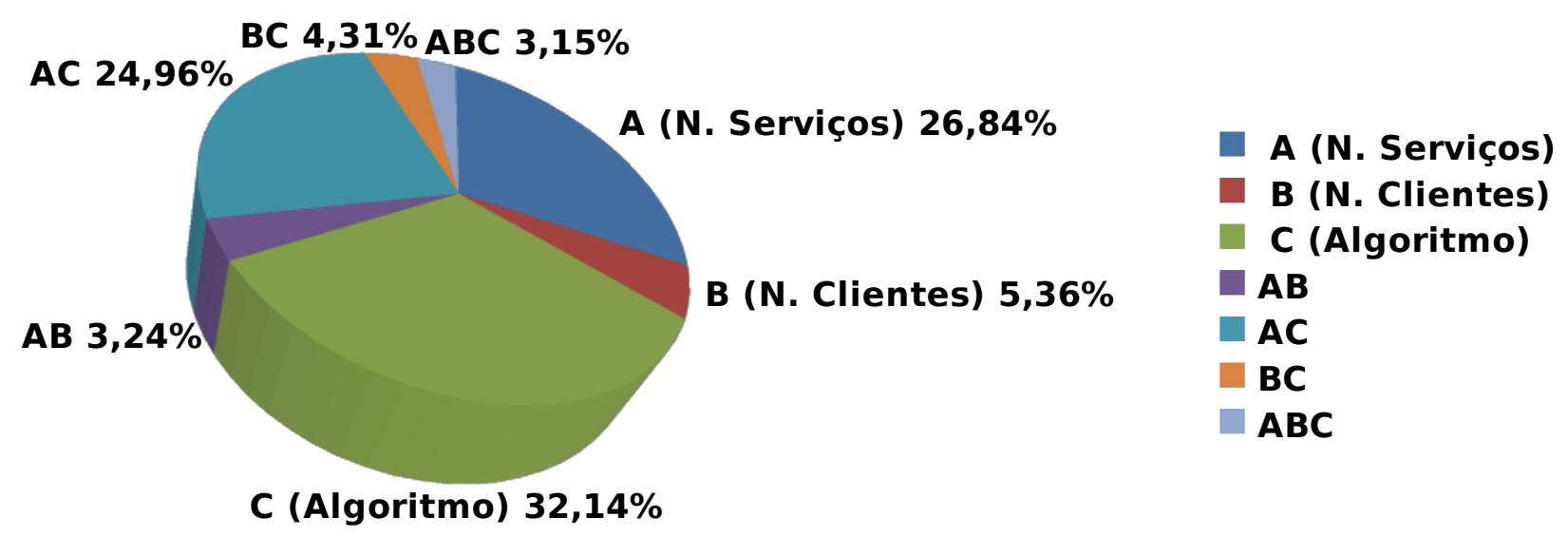

Figura 6.18: Porcentagem de Influência de cada fator no tempo de busca (TB) do módulo durante os experimentos (CJE-2).

sistema. As outra combinações $\mathrm{BC}(4,31 \%), \mathrm{AB}(3,24 \%)$ e $\mathrm{ABC}(3,15 \%)$ compõe a porcentagem restante da influência do conjunto de experimento 2 .

Da mesma forma que o conjunto de experimento 1 (CJE-1), a Monitoração de Carga (MC) estava inativa (OFF), portanto não há resultados sobre a carga de processamento no módulo.

\subsubsection{Conjunto de Experimentos 3}

O conjunto de experimentos 3 (CJE-3) é similar ao primeiro conjunto de experimentos (CJE-1) e apenas se diferenciam quanto ao fator fixo: Número de Atributos de QoS (AQ). No CJE-1 foram utilizados 3 (três) atributos de QoS para classificar os serviços como Gold, Silver e Bronze. Por outro lado, no CJE-3 foram utilizados apenas 2 (dois) atributos conforme exibe a Tabela 6.9.

Os resultados desse terceiro conjunto de experimento (CJE-3) são estatisticamente iguais aos resultados dos primeiro conjunto de experimento (CJE-1). Portanto, seria repetitiva a exibição de gráficos e resultados deste experimento. Devido a essa igualdade estatística pode-se afirmar que a 
adição (ou a remoção) de apenas um atributo de QoS não exerce influência significativa nos experimentos efetuados. Contudo, a possível adição de vários atributos poderá exercer mais influência, principalmente no processo de inferência por parte do reasoner. No entanto, a realização de novos experimentos para averiguar essa suposição é proposta para trabalhos futuros.

Para exemplificar a igualdade estatística, a Figura 6.19 exibe uma comparação entre alguns resultados dos CJE-1 e CJE-3. Analisando os gráficos dessa Figura 6.19 é possível notar que para qualquer que seja o algoritmo utilizado com 30 clientes e 600 serviços (número máximo de clientes e serviços) as médias obtidas em cada conjunto de experimentos estão sobrepostas aos intervalos de confiança.

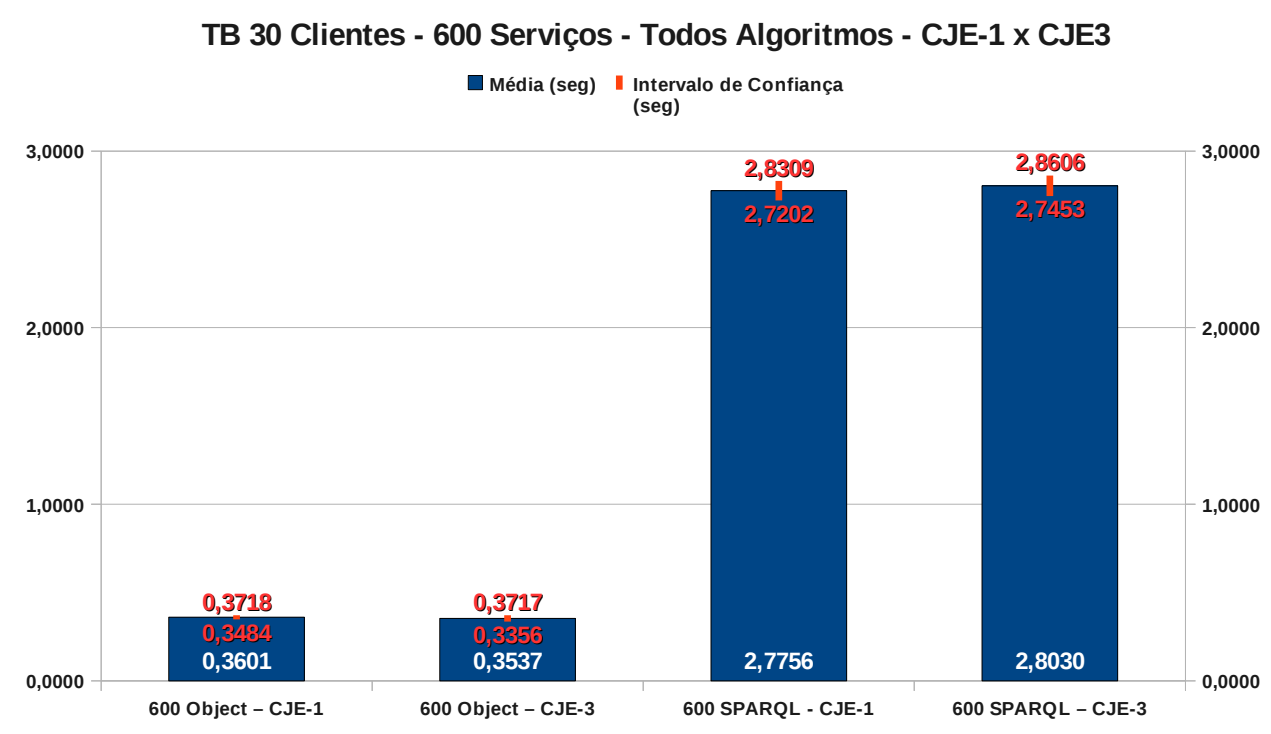

Figura 6.19: Comparação no Tempo de Busca (TB) dos CJE-1 e CJE-3 utilizando os algoritmos com 30 clientes e 600 serviços.

A Figura 6.20 apresenta os resultados do cálculo da influência da variável de resposta Tempo de Busca (TB) desse terceiro conjunto de experimento. Os resultados da influência dos fatores neste CJE-3 são muito próximos aos resultados do CJE-1 devido à igualdade estatística. Além disso, uma tabela com a completude dos resultados obtidos no CJE-3 encontra-se no Anexo 4 desta monografia. 
BC $6,64 \%$ ABC $1,39 \%$

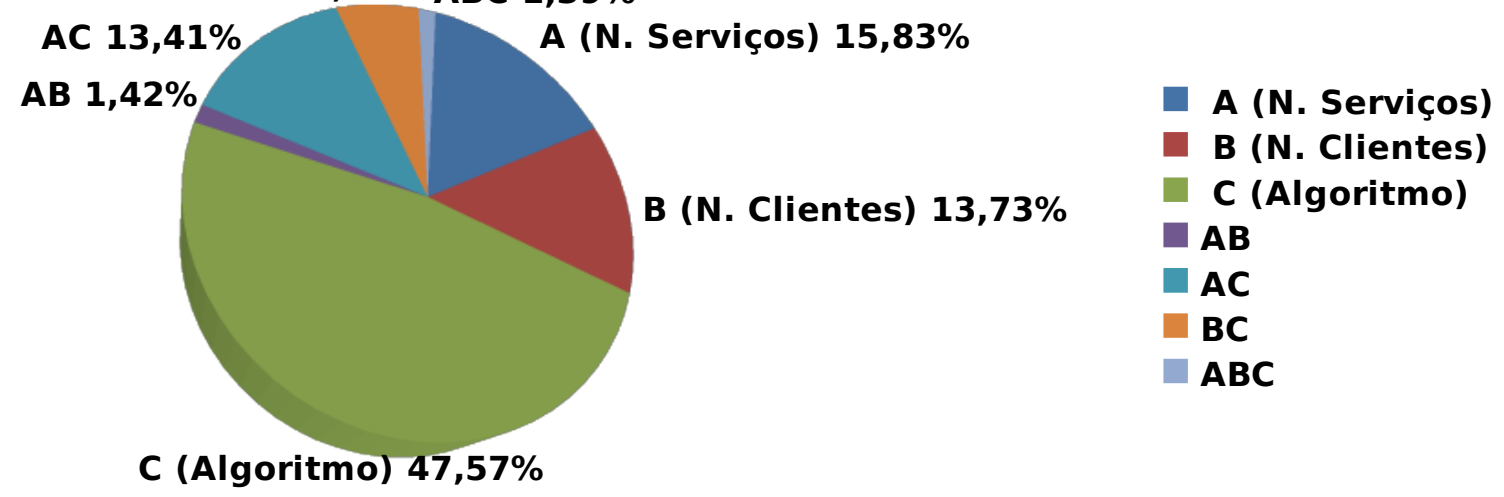

Figura 6.20: Porcentagem de Influência de cada fator no tempo de busca (TB) do módulo durante os experimentos (CJE-3).

A Monitoração de Carga (MC) estava inativa (OFF) neste conjunto de experimento. Portanto, não há resultados sobre a carga de processamento imposta pelo módulo para serem analisados.

\subsubsection{Conjunto de Experimentos 4}

O mesmo comportamento que ocorreu com o conjunto de experimentos 3 (CJE-3) em relação ao CJE-1 se repetiu para o conjunto de experimento 4 (CJE-4) em relação ao CJE-2. Os resultados obtidos foram estatisticamente iguais utilizando-se 3 (três) ou 2 (dois) atributos de QoS no fator fixo: Número de Atributos de QoS (AQ).

Novamente para exemplificar, os resultados obtidos com os dois algoritmos utilizando-se os maiores níveis dos fatores (30 clientes e 1200 serviços) foram comparados. Esses resultados, em escala logarítmica, estão ilustrados no gráfico da Figura 6.21. Análogo aos resultados da Figura 6.19, os resultados nessa Figura 6.21 demostram que as médias obtidas para os experimentos de mesmo algoritmo estão muito próximas e sobrepostas aos intervalos de confiança, mesmo em conjunto de experimentos diferentes. 


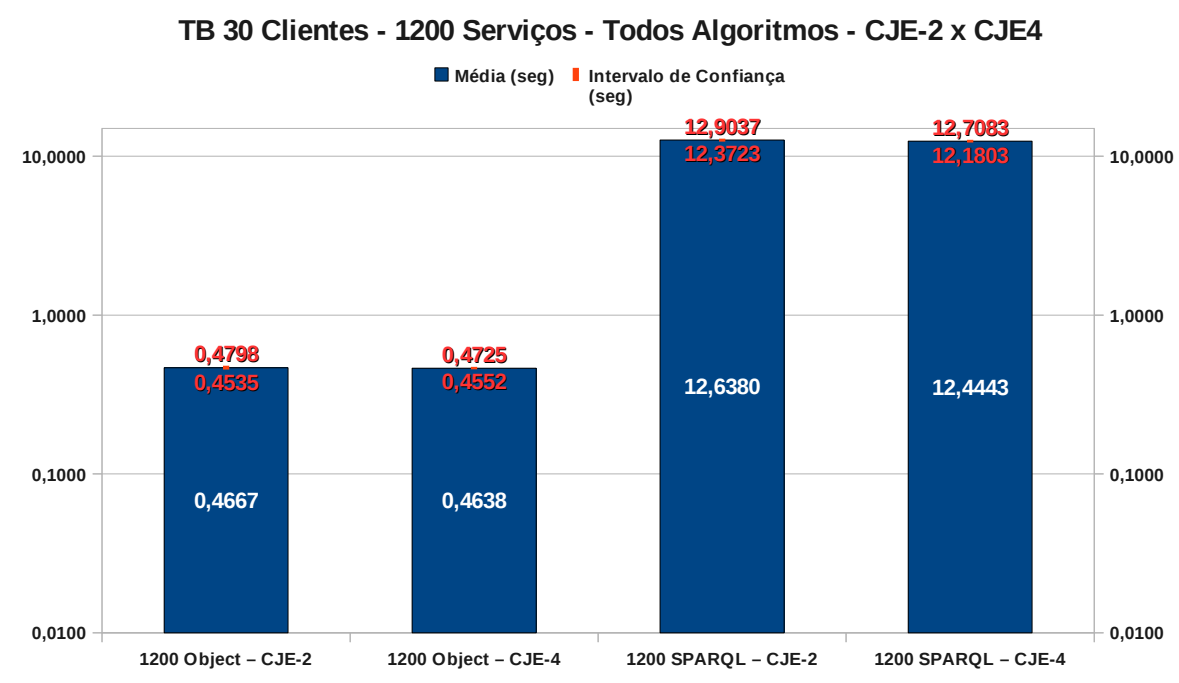

Figura 6.21: Comparação no Tempo de Busca (TB) dos CJE-2 e CJE-4 utilizando os algoritmos com 30 clientes e 1200 serviços.

A Figura 6.22 exibe os resultados dos cálculos da influência da variável de resposta Tempo de Busca (TB) desse quarto conjunto de experimentos. A influência dos fatores neste CJE-4 é quase a mesma do CJE-2 por causa da igualdade estatística entre eles. No Anexo 4 desta monografia há uma tabela com a completude dos resultados obtidos no CJE-4.

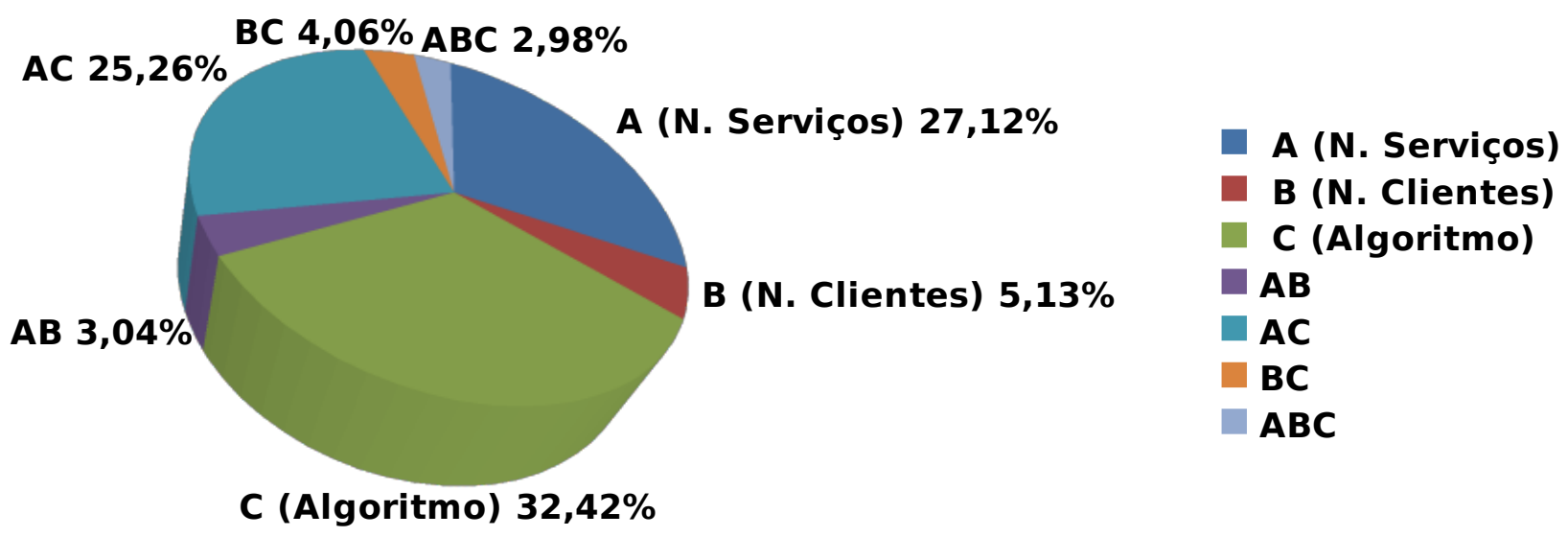

Figura 6.22: Porcentagem de Influência de cada fator no tempo de busca (TB) do módulo durante os experimentos (CJE-4).

O fator fixo Monitoração de Carga (MC) continuou inativo (OFF) neste conjunto de experimento. Dessa forma, não foi possível coletar dados sobre a carga de processamento imposta pelo módulo. 


\subsubsection{Conjunto de Experimentos 5}

O quinto conjunto de experimentos (CJE-5) manteve os mesmos fatores e níveis do CJE-3 . O que diferencia o CJE-3 do CJE-5 é que neste último a opção de monitoramento dos provedores estava ativada. Além disso, um script em perl permaneceu em execução no servidor do módulo neste quinto conjunto de experimentos com o objetivo de monitorar o consumo médio de processamento durante a descoberta e a seleção de serviços com qualidade.

Dessa forma, todos os fatores fixos neste conjunto de experimentos estavam ativos em concordância com a Tabela 6.9. Devido a ativação desses fatores os resultados obtidos não foram estatisticamente iguais aos resultados dos conjuntos de experimentos 1 e 3 . O monitoramento de carga pelo script escrito em perl não acrescenta uma grande sobrecarga ao sistema, porém mantém o processador sempre ativo. Por outro lado, o monitoramento dos provedores exige um processamento extra para o módulo. O sistema deve consultar periodicamente o estado dos provedores utilizando o Ganglia. Além disso, o módulo deve consultar tais informações com o objetivo de selecionar o melhor provedor naquele determinado momento. Essa seleção é feita em todas as requisições dos clientes, pois sempre haverá mais de um serviço compatível com a qualidade exigida pelo cliente.

É importante relembrar que os fatores que variam neste CJE-5 são os mesmos dos CJE-1, ou seja, foram utilizados 300 e 600 serviços (Fator A), 15 e 30 clientes (Fator B) e os dois algoritmos (Fator C).

A Figura 6.23 exibe a variação no tempo de busca (TB) quando o número de serviços é alterado de 300 para 600 serviços com 15 requisições simultâneas. Os resultados indicam que o comportamento foi o mesmo do CJE-1 com a diferença das médias dos tempos de buscas serem um pouco maiores. Esse resultado era esperado, pois no CJE-5 há uma sobrecarga extra em razão da monitoração e seleção dos provedores a cada requisição.

TB 15 Clientes - 300x600 Serviços - OntAlgorithmObject x OntAlgorithmSPARQL

प Média (seg) IIntervalo de Confiança (seg)

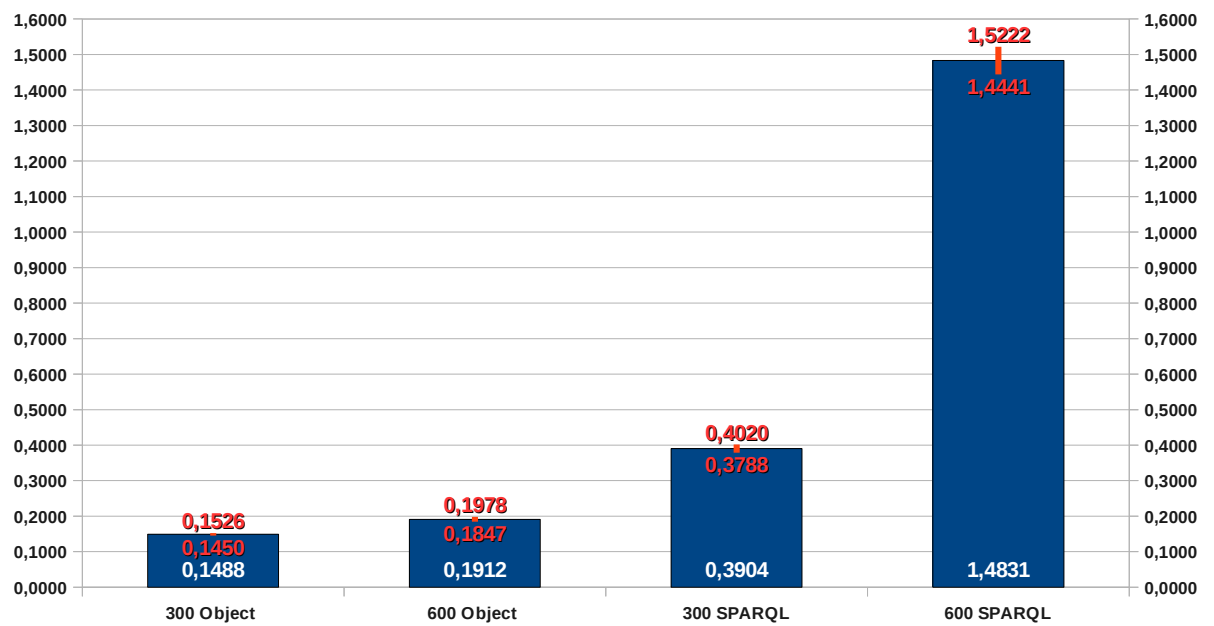

Figura 6.23: Tempo de Busca (TB) utilizando os algoritmos com 15 clientes (CJE-5). 
Entretanto, o resultado do tempo médio de busca com 15 clientes, 300 serviços e algoritmo OntAlgorithmSPARQL, em específico, apresentou um tempo de busca menor em comparação ao seu correspondente no CJE-1 (Figura 6.24).

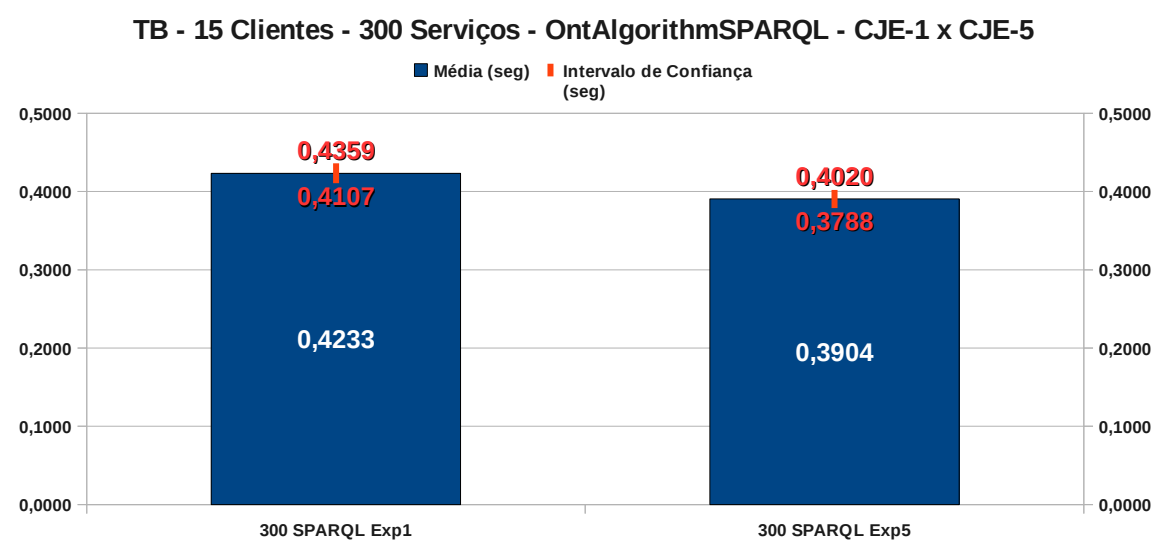

Figura 6.24: Comparação do Tempo de Busca (TB) entre o CJE-1 x CJE-5 utilizando 15 clientes, 300 serviços e o algoritmo OntAlgorithmSPARQL.

Uma explicação plausível para esse fato é devida à configuração de economia de energia adotada. A versão do sistema operacional utilizada nos experimentos permite que um gerenciador regule as frequências disponíveis do processador de acordo com políticas de energia/desempenho pré-definidas. Por exemplo, o regulador powersave configura estatisticamente o processador para trabalhar na menor frequência disponível. Por outro lado, o regulador performance faz o contrário e configura o processador na maior frequência disponível. A partir do kernel 2.6.10, o regulador padrão é o ondemand, que foi adicionado com a capacidade de alterar a frequência do processador dinamicamente de acordo com a sua utilização (Hopper, 2009). Há outros reguladores (politicas) de gerenciamento de energia disponíveis (Hopper, 2009). Porém o regulador ondemand é o foco de interesse para justificar o resultado obtido.

$\mathrm{O}$ regulador ondemand configura em princípio a menor frequência disponível e permanece monitorando a utilização do processador. Caso a utilização ultrapasse o limite, o regulador deve selecionar a frequência mais elevada disponível. Caso a monitoração indique que a utilização está abaixo do limite, então o regulador irá diminuir a frequência para a próxima disponível. Se o processador ainda continuar sendo subutilizado, novamente o regulador diminuirá a frequência até que se atinja a menor frequência disponível (Hopper, 2009).

A máquina que hospeda o módulo UDOnt-Q dispõe de um processador (Intel QuadCore Q9400) cuja arquitetura permite a utilização de duas frequências $(2.00 \mathrm{GHz}$ e $2.66 \mathrm{GHz})$. Na configuração de gerenciamento de energia padrão, ou seja, empregando o regulador ondemand, os experimentos que exigiam menor processamento (experimentos com 300 e 600 serviços e utilizando o algoritmo OntAlgorithmObject) conseguiram ser atendidos rapidamente sem atingir o limite de processamento $(2.00 \mathrm{GHz})$ imposto para a troca de frequência. Contudo, a partir da utilização do algoritmo OntAlgoritmoSPARQL, agregando a monitoração e seleção dos provedores, o limite foi ultrapassado em algum momento e a frequência do processador foi alterada pelo regulador para a maior 
(2.66GHz). Devido a esse aumento de frequência o tempo de busca, em média, foi menor do que o mesmo tempo correspondente no conjunto de experimento 1 (CJE-1).

Para os demais resultados que utilizam o algoritmo OntAlgoritmoSPARQL o tempo de busca médio no CJE-5 foi maior pois mesmo que tenha ocorrido um aumento na frequência do processador, esse aumento não foi suficiente para compensar a carga de imposta (600 ou 1200 serviços e/ou 30 clientes) ao ponto de diminuir ou igualar o tempo de busca.

As afirmações do parágrafo anterior são as possíveis causas que justificam o resultado para um tempo de busca menor. Essa "perturbação" no ambiente de testes não estava prevista e, portanto a frequência do processador durante os experimentos não foi registrada. Contudo, as taxas de utilização dos processadores foram coletadas e o comportamento dos resultados na Figura 6.29 reforça a hipótese defendida para tal resultado. O comportamento e os resultados da Figura 6.29 serão explicados mais adiante nesta subseção.

Nos resultados apresentados na Figura 6.25 foram utilizados 30 clientes simultâneos ao invés de apenas 15. Entretanto, não houve problemas com o experimento com a configuração de 30 clientes, 300 serviços e o algoritmo OntAlgorithmSPARQL. Provavelmente a troca de frequência favoreceu no tempo médio de busca, mas neste caso não foi suficiente para obter um tempo menor ou igual ao seu correspondente no CJE-1.

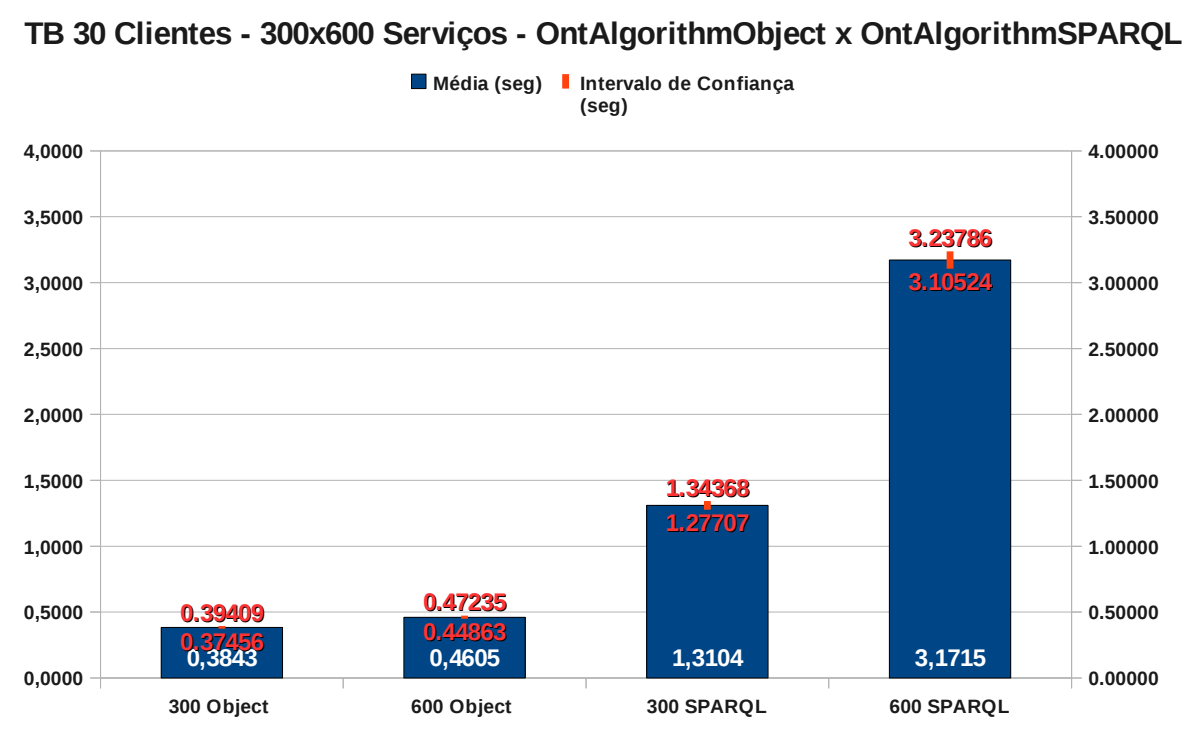

Figura 6.25: Tempo de Busca (TB) utilizando os algoritmos com 30 clientes (CJE-5).

Novamente, o comportamento com 30 clientes foi análogo ao comportamento do CJE-1, porém os tempos médios aumentaram devido à mesma sobrecarga extra que foi discutida anteriormente. As análises de desempenho desse quinto conjunto de experimentos (CJE-5) refletem àquelas feitas no primeiro (CJE-1). Por exemplo, nota-se que o algoritmo OntAlgorithmObject é mais eficiente que o OntAlgorithSPARQL. Além disso, o fato de dobrar o número de clientes manteve um aumento acima de $100 \%$ nos tempos de buscas obtidos. 
Os resultados dos tempos de resposta nos clientes (RTC) seguiram o mesmo padrão do tempo de busca (TB). Relembrando que o RTC envolve a soma do tempo de busca, o tempo de transferência de dados pela rede e o tempo de processamento para empacotamento (parser) e desempacotamento da mensagem SOAP.

A Figura 6.26 exibe as médias dos tempos de respostas obtidos nas repetições do experimento utilizando 15 clientes, 300 serviços, 600 serviços e ambos os algoritmos.

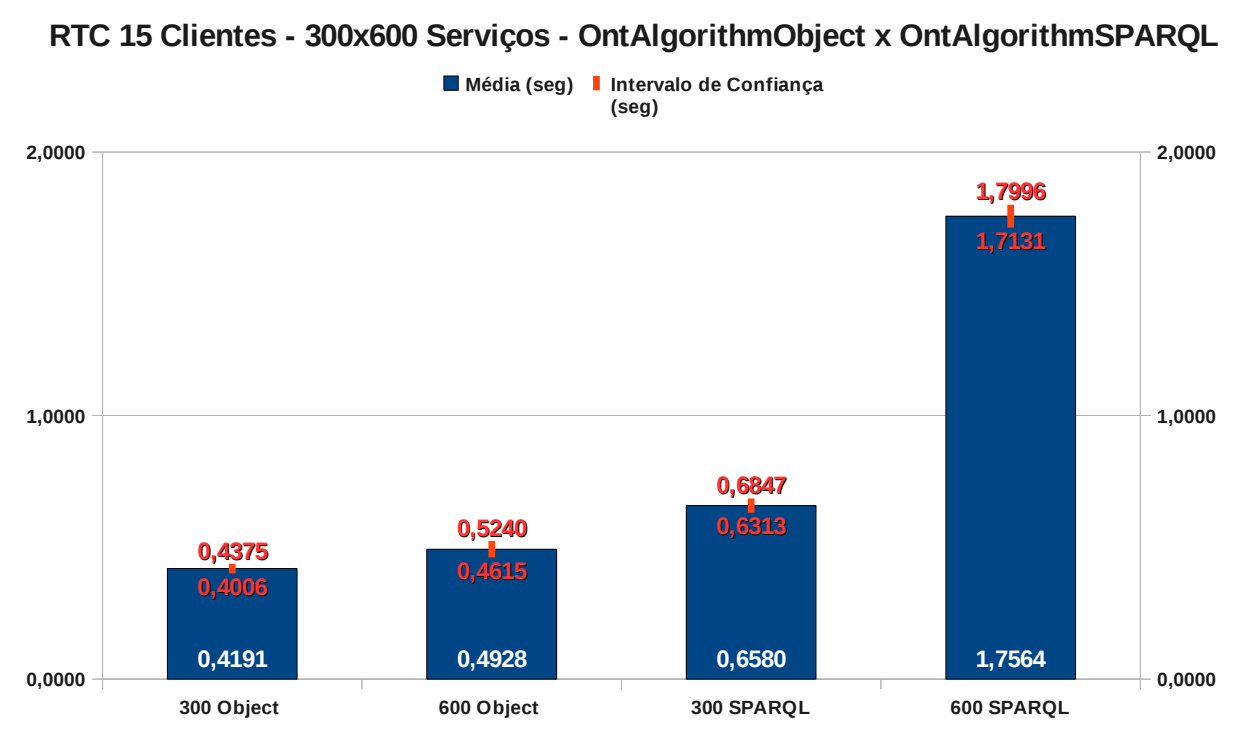

Figura 6.26: Tempo de Resposta para o Cliente (RTC) utilizando os algoritmos com 15 clientes (CJE-5).

A Figura 6.27 complementa os resultados anteriores e exibe os tempos de resposta nos clientes (RTC) sendo considerados 30 clientes concorrentes

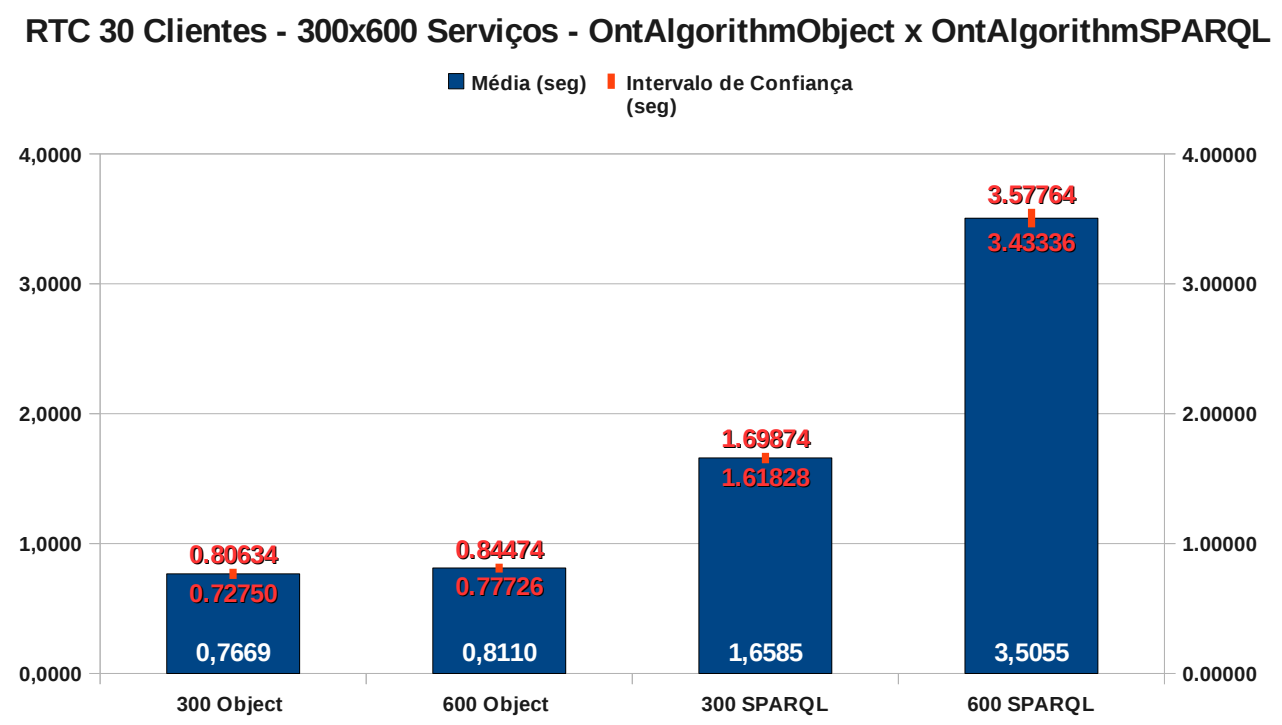

Figura 6.27: Tempo de Resposta para o Cliente (RTC) utilizando os algoritmos com 30 clientes (CJE-5). 
Analisando e comparando os resultados das Figuras 6.26 e 6.27, nota-se um aumento no tempo médio de resposta quando se adotou 30 clientes. Essa conduta é semelhante ao que ocorreu no conjunto de experimentos 1 (CJE-1).

A Tabela 6.12 lista as possibilidades do fatorial completo $2^{3}$ das médias dos tempos de busca (TB), as médias dos tempos de resposta para os clientes (RTC). A partir dessas médias são calculadas as vazões (Throughput). Na Figura 6.28 é possível observar o resultado do cálculo da influência dos fatores para a variável de resposta Tempo de Busca (TB) do CJE-5.

Tabela 6.12: Fatorial completo $2^{3}$ e Médias do Tempo de Busca (TB) do CJE-5

\begin{tabular}{|c|c|c|c|c|c|c|}
\hline Experimento & $\begin{array}{c}\text { Fator A } \\
\text { (N. Serviços) }\end{array}$ & $\begin{array}{c}\text { Fator B } \\
\text { (N. Clientes) }\end{array}$ & $\begin{array}{c}\text { Fator C } \\
\text { (Algoritmo) }\end{array}$ & TB Média & RTC Média & Throughput \\
\hline 1 & 300 & 15 & Object & 0,14883 & 0,41908 & 2,39 \\
\hline 2 & 300 & 15 & SPARQL & 0,39042 & 0,65803 & 1,52 \\
\hline 3 & 300 & 30 & Object & 0,38433 & 0,76692 & 1,3 \\
\hline 4 & 300 & 30 & SPARQL & 1,31037 & 1,65851 & 0,6 \\
\hline 5 & 600 & 15 & Object & 0,19123 & 0,49277 & 2,03 \\
\hline 6 & 600 & 15 & SPARQL & 1,48313 & 1,75636 & 0,57 \\
\hline 7 & 600 & 30 & Object & 0,46049 & 0,811 & 1,23 \\
\hline 8 & 600 & 30 & SPARQL & 3,17155 & 3,5055 & 0,29 \\
\hline
\end{tabular}

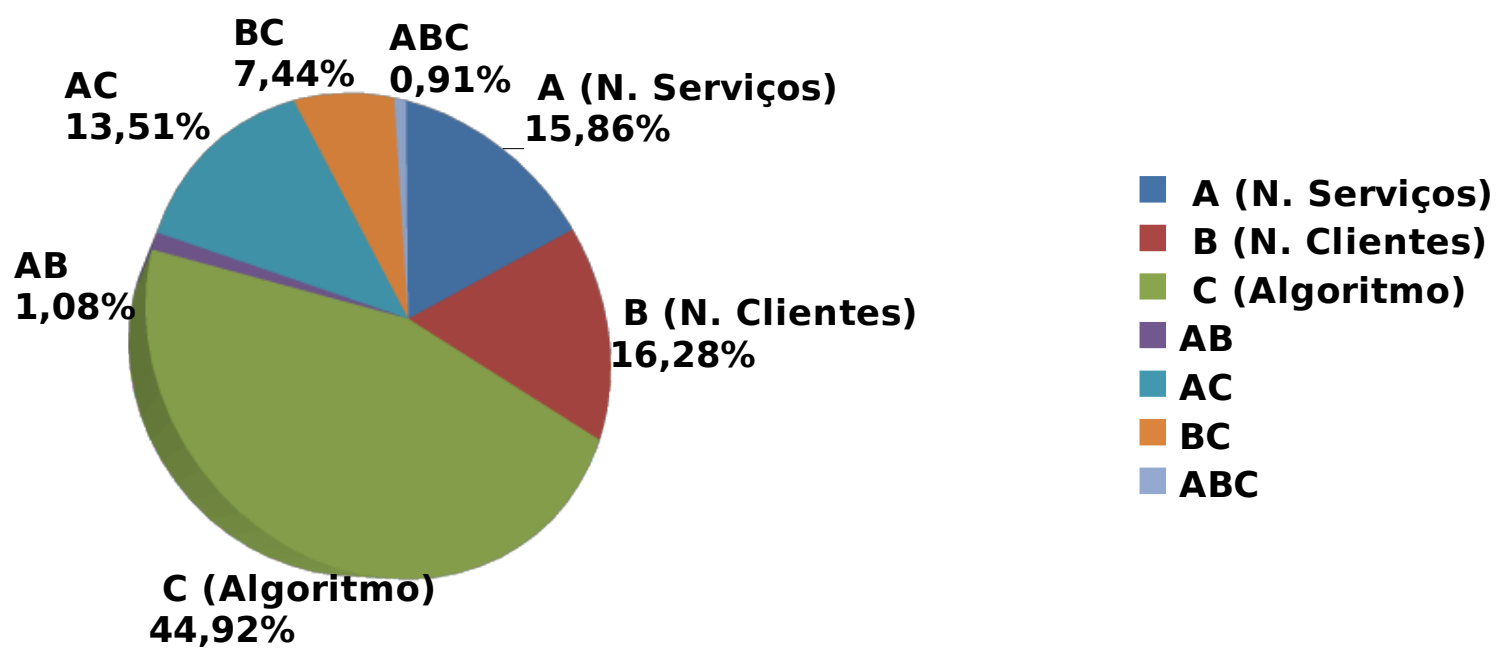

Figura 6.28: Porcentagem de Influência de cada fator no tempo de busca (TB) do módulo durante os experimentos (CJE-5).

Analisando-se as porcentagens de influência de cada fator da Figura 6.28, observa-se que o fator C (Algoritmo), com 44,92\% de influência, é o que está com a maior porcentagem nos experimentos. Durante todos os conjuntos de experimentos, o fator C (Algoritmo) tem apresentado a maior influência nos cálculos. A variação no tempo de busca quando os algoritmos são trocados é maior que qualquer variação dos outros fatores. No CJE-1 o fator A obteve uma influência um pouco maior com $48,46 \%$. 
O segundo fator em ordem de influência neste CJE-5, com 16,28\%, foi o fator B (Número de Clientes), seguido pelo fator A (Número de Serviços) com 15,86\% de influência. É possível observar que houve uma inversão da posição entre esses fatores em comparação com o CJE-1. No CJE-5 o fator B ficou a frente do fator A por causa da Monitoração pelo Ganglia (MG) que estava ativa. Devido a essa monitoração, cada requisição do cliente exigia um tempo e processamento extra para monitorar e selecionar os provedores adequados naquele instante. Esse fato aumentou a influência do fator B (Número de clientes/Número de requisições) no sistema.

As interações entre fatores complementam o restante da porcentagem da influência, a interação $\mathrm{AC}$ ficou com 13,51\%, BC com 7,44\%, AB com 1,08\% e ABC com 0,91\%.

Neste quinto conjunto de experimento (CJE-5), o fator fixo Monitoração de Carga (MC) estava ativo (ON). Neste caso, foi possível coletar dados sobre a carga de processamento imposta pelo módulo. Esses dados foram analisados gerando os resultados da Figura 6.29.

A análise dos resultados da carga de processamento indica que é preciso uma maior taxa de processamento para o algoritmo OntAlgorithmSPARQL. Enquanto o consumo dos processadores com o algoritmo OntAlgorithmObject não ultrapassa a taxa de $20 \%$, com o algoritmo OntAlgorithmSPARQL essa taxa inicia em quase $30 \%$ e chega próximo dos $70 \%$. O OntAlgorithmObject é mais eficiente para as cargas impostas e os resultados de vazão apresentados na Tabela 6.12 endossam essa afirmação.

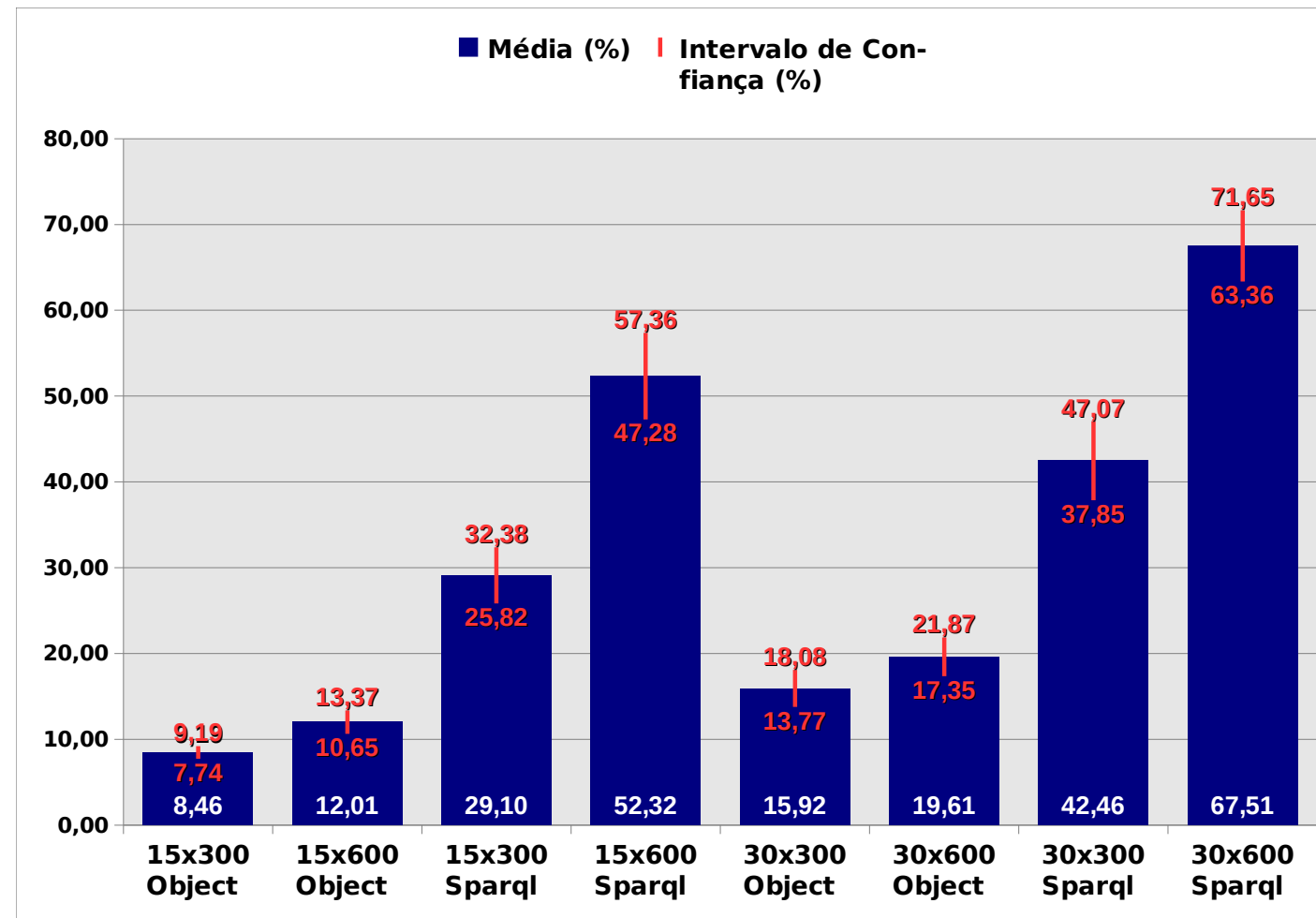

Figura 6.29: Porcentagem da carga de processamento (CPU-Load) do módulo durante os experimentos (CJE-5). 


\subsubsection{Conjunto de Experimentos 6}

No sexto conjunto de experimentos comparado com o CJE-5 há a diferença que o fator A possui 300 e 1200 serviços ao invés de 300 e 600 serviços. Os fatores fixos, Gravação de Log (GL), Monitoração pelo Ganglia (MG) e Monitoração de Carga (MC) permaneceram ativos. Dessa forma, esse conjunto de experimentos 6 seguiu a configuração da Tabela 6.9.

Os resultados dos tempos de busca (TB) com 15 clientes desse conjunto de experimentos são apresentados na Figura 6.30 em escala logarítmica.

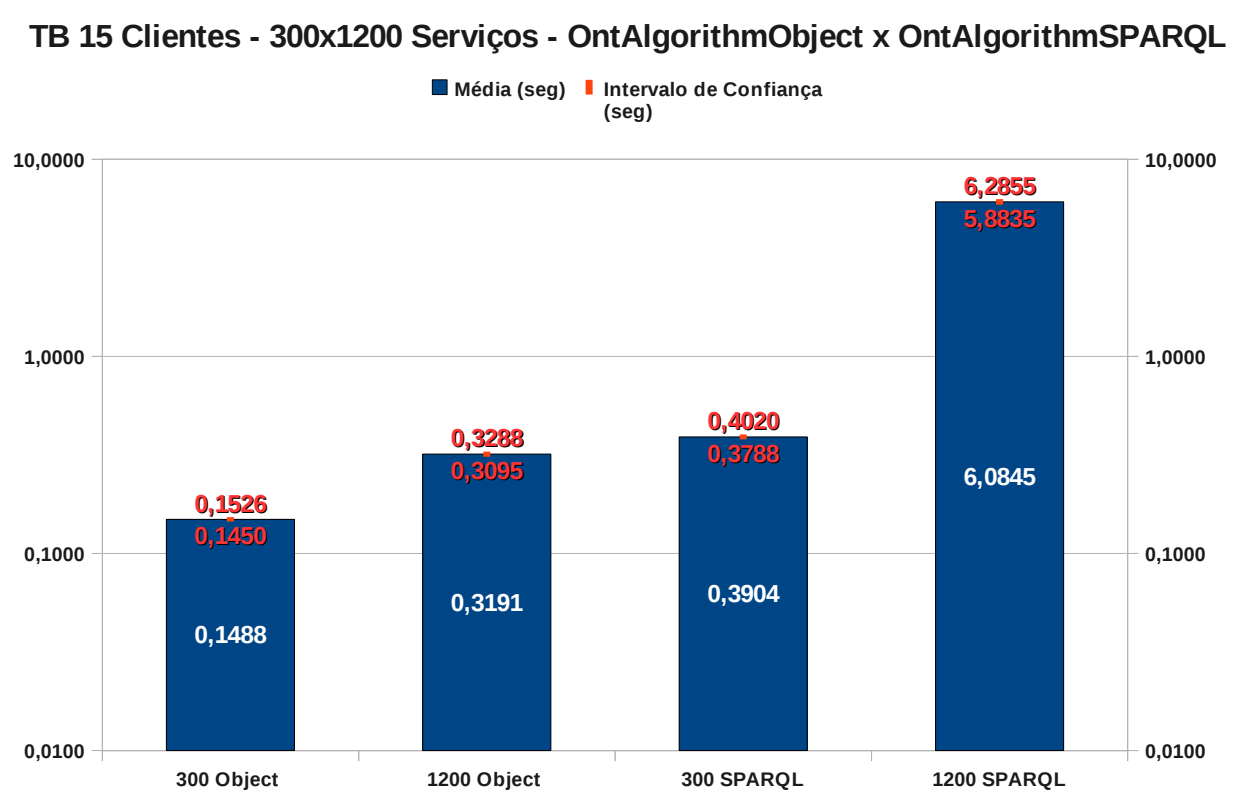

Figura 6.30: Tempo de Busca (TB) utilizando os algoritmos com 15 clientes (CJE-6).

É possível afirmar que os resultados dos tempos de busca com 15 clientes desse CJE-6 são maiores que os tempos de busca do CJE-2, com exceção do experimento com 1200 serviços e o algoritmo OntObjectSPARQL. Comparando ambos os experimentos em seus respectivos conjuntos, observam-se que as médias dos resultados obtidos estão sobrepostas aos intervalos de confiança. Isso indica que o fato das monitorações estarem ativas não foi suficiente para superar a grande variação de tempo exercida neste experimento em especifico. Portanto, a sobrecarga gerada pelas monitorações aumenta os tempos médios de busca, porém não é possível afirmar que eles são estatisticamente diferentes considerando um intervalo de confiança de $95 \%$.

Por outro lado, utilizando-se a escala logarítmica, a Figura 6.31 exibe os resultados com 30 clientes concorrentes deste CJE-6 e apresenta um tempo de busca (TB) maior no experimento com 1200 serviços e o algoritmo OntObjectSPARQL do que o seu correspondente no CJE-2. Isso significa que, com 30 clientes a sobrecarga imposta pelas monitorações teve um efeito mais significativo no tempo de busca (TB), pois, o número de requisições é diretamente proporcional à quantidade de vezes que o módulo precisa analisar e selecionar o melhor provedor. Ou seja, quanto 
maior o número de requisições, maior será a sobrecarga no módulo e, consequentemente, maior será o tempo de busca.

Os resultados dos tempos de buscas apresentados nas Figuras 6.30 e 6.31 estão proporcionalmente de acordo os resultados do CJE-2, confirmando as análises que indicam o algoritmo OntAlgorithmObject como o mais eficiente e que a variação do número de clientes e de serviços proporciona um aumento não linear nos resultados.

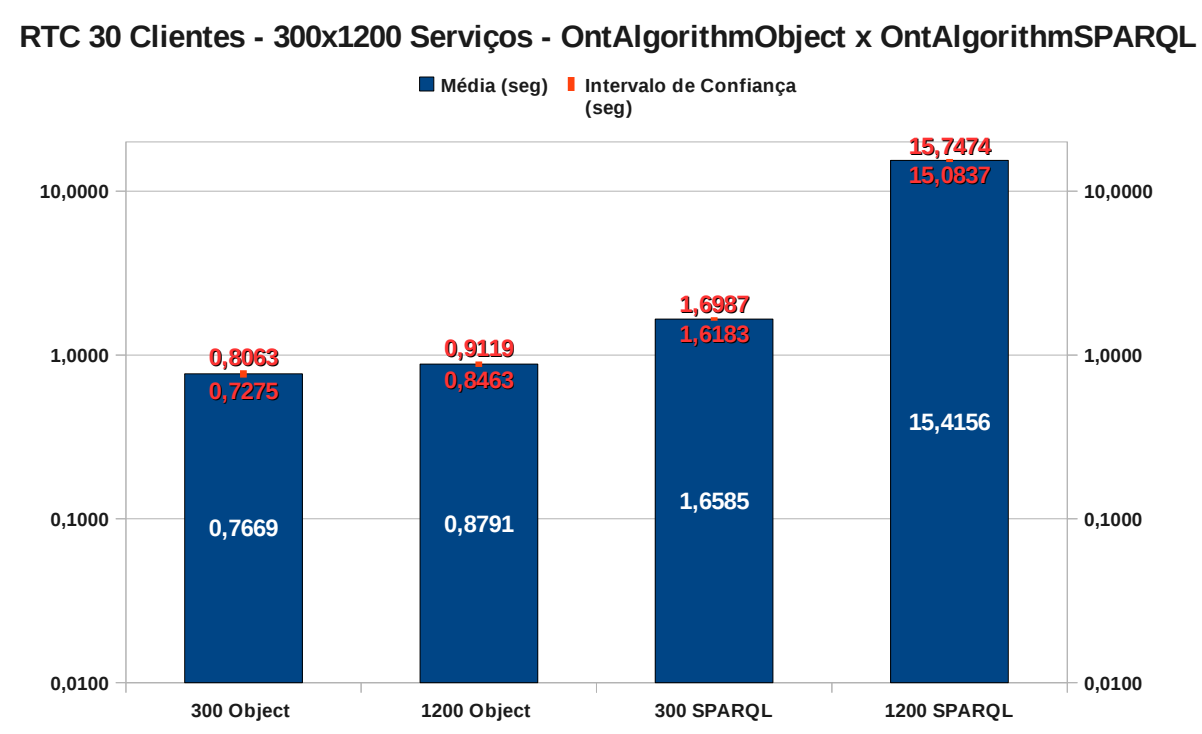

Figura 6.31: Tempo de Busca (TB) utilizando os algoritmos com 30 clientes (CJE-6).

Os resultados dos tempos de resposta para os clientes (RTC) estão exibidos na Figura 6.32. É importante lembrar que esse tempo envolve principalmente o tempo de transmissão das informações pela rede e o tempo de processamento das mensagens SOAP, tanto do lado do módulo como do lado do cliente. Os tempos médios de resposta para os clientes neste sexto conjunto de experimentos são maiores que os seus correspondentes no CJE-2. Dessa forma, a análise estatística indica que de acordo com os resultados obtidos nestes conjuntos de experimentos, pode-se afirmar que eles são estatisticamente diferentes.

Os resultados de RTC com 15 clientes de cada experimento (Figura 6.32) também são estatisticamente diferentes.

Na Figura 6.33 são exibidos os tempos de respostas com 30 clientes simultâneos. O comportamento e a análise desses resultados são praticamente os mesmos dos resultados com 15 clientes. Porém, o tempo médio de resposta para o cliente foi maior em cada um dos experimentos.

A Tabela 6.13 lista o fatorial completo $2^{3}$ das médias dos tempos de busca (TB) do módulo, as médias dos tempos de resposta para os clientes (RTC) e a partir dessas últimas médias são calculadas as vazões (Throughput). 
RTC 15 Clientes - 300x1200 Serviços - OntAlgorithmObject x OntAlgorithmSPARQL

$\square$ Média (seg) I Intervalo de Confiança (seg)

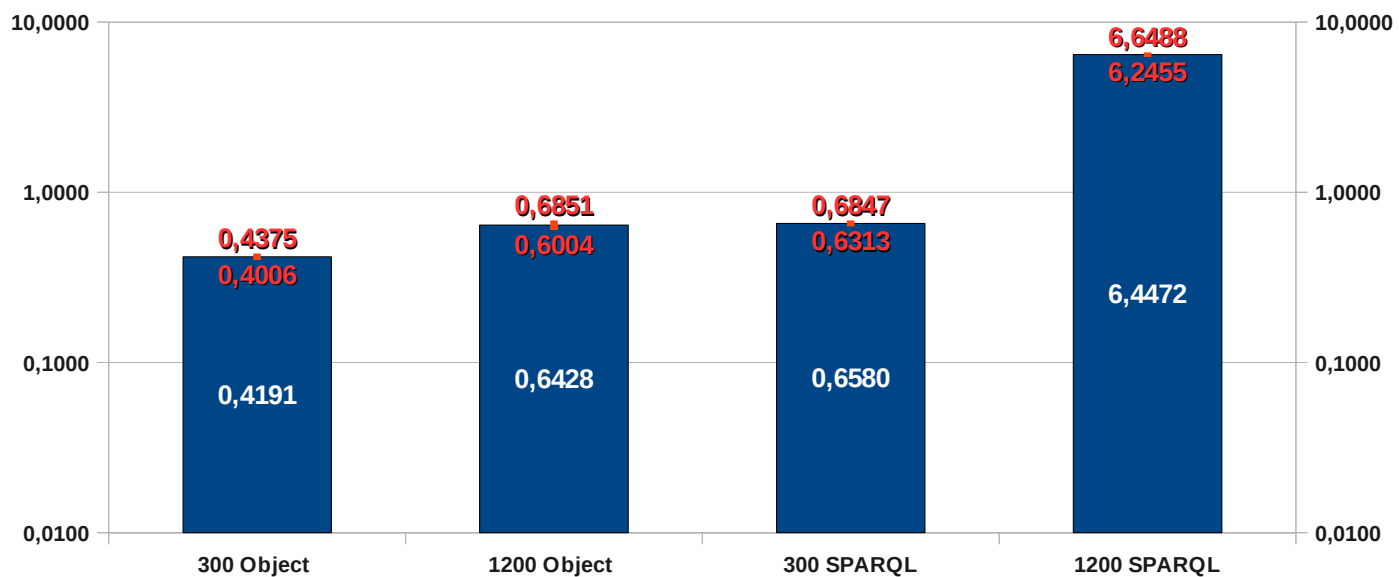

Figura 6.32: Tempo de Resposta para o Cliente (RTC) utilizando os algoritmos com 15 clientes (CJE-6).

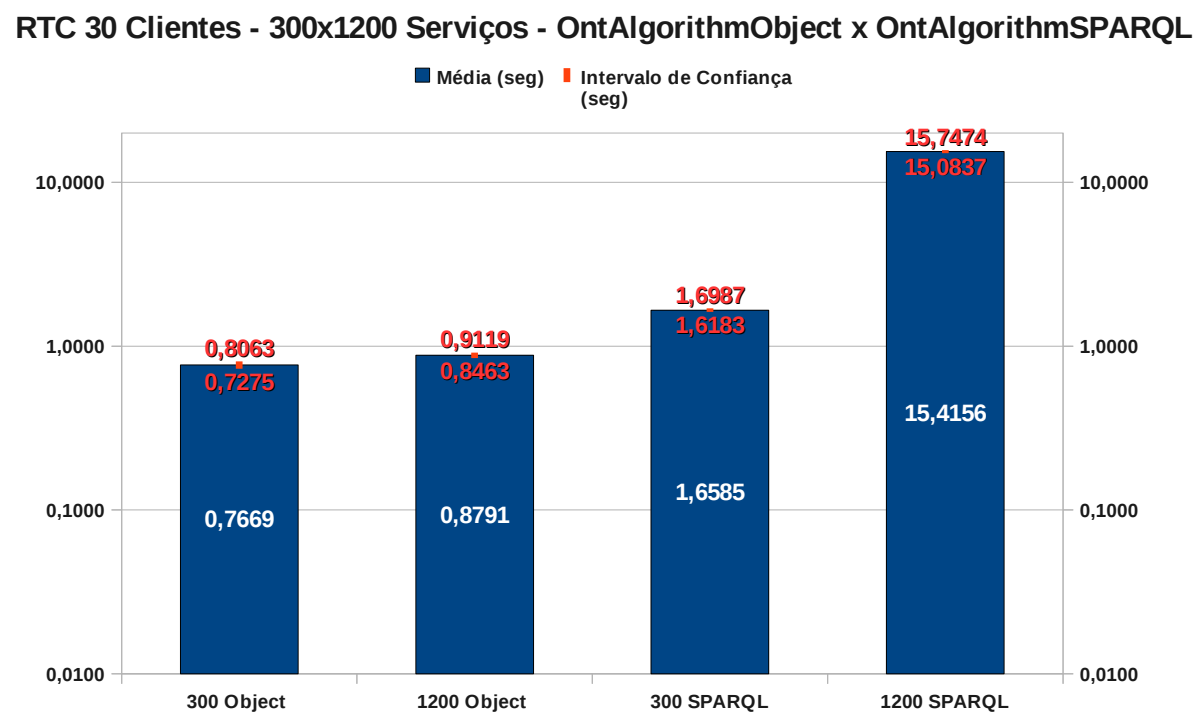

Figura 6.33: Tempo de Resposta para o Cliente (RTC) utilizando os algoritmos com 30 clientes (CJE-6).

As porcentagens de influência de cada fator durante a execução dos experimentos na busca por serviços são exibidas na Figura 6.34.

Os resultados apresentados na Tabela 6.13 resumem as observações discutidas no decorrer desta subseção. A porcentagem de influência de cada fator foi calculada, conforme exibe a Figura 6.34. Em concordância com os resultados dos outros conjuntos de experimentos, neste sexto conjunto o fator $\mathrm{C}$ (Algoritmo) foi novamente o que mais influencia nos experimentos executados. Esse fator obteve $29,84 \%$ de influência ficando à frente do fator A (Número de Serviços) com $25,39 \%$ e a interação entre fatores $\mathrm{AC}$ com $23,68 \%$. Ao contrário do que houve com a influência 
Tabela 6.13: Fatorial completo $2^{3}$ e Médias do Tempo de Busca (TB) do CJE-6

\begin{tabular}{|c|c|c|c|c|c|c|}
\hline Experimento & $\begin{array}{c}\text { Fator A } \\
\text { (N. Serviços) }\end{array}$ & $\begin{array}{c}\text { Fator B } \\
\text { (N. Clientes) }\end{array}$ & $\begin{array}{c}\text { Fator C } \\
\text { (Algoritmo) }\end{array}$ & TB Média & RTC Média & Throughput \\
\hline 1 & 300 & 15 & Object & 0,14883 & 0,4191 & 2,39 \\
\hline 2 & 300 & 15 & SPARQL & 0,39042 & 0,658 & 1,52 \\
\hline 3 & 300 & 30 & Object & 0,38433 & 0,7669 & 1,3 \\
\hline 4 & 300 & 30 & SPARQL & 1,31037 & 1,6585 & 0,6 \\
\hline 5 & 1200 & 15 & Object & 0,31912 & 0,6428 & 1,56 \\
\hline 6 & 1200 & 15 & SPARQL & 6,08451 & 6,4472 & 0,16 \\
\hline 7 & 1200 & 30 & Object & 0,55112 & 0,8791 & 1,14 \\
\hline 8 & 1200 & 30 & SPARQL & 15,03554 & 15,4156 & 0,06 \\
\hline
\end{tabular}

no CJE-5, o fator A manteve a segunda posição. Esse resultado era previsto, pois o número de serviços entre os conjuntos de experimentos 5 e 6 são diferentes, enquanto no CJE-5 a avaliação considerou até 600 serviços, o CJE-6 considerou até 1200 serviços.

\section{BC $5,75 \% \quad$ ABC $4,20 \%$}

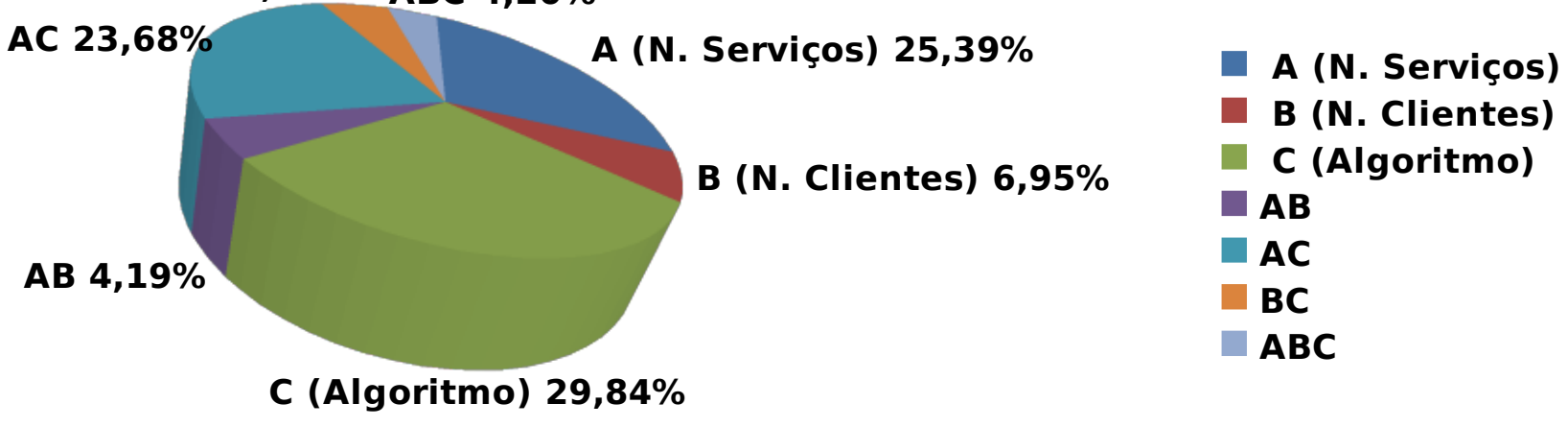

Figura 6.34: Porcentagem de Influência de cada fator no tempo de busca (TB) do módulo durante os experimentos (CJE-6).

O fator fixo Monitoração de Carga (MC) estava ativo (ON) neste conjunto de experimentos. Os resultados sobre a carga de processamento imposta pelo módulo durante os experimentos estão exibidos na Figura 6.35. 


\section{Média (\%) | Intervalo de Con- fiança (\%)}

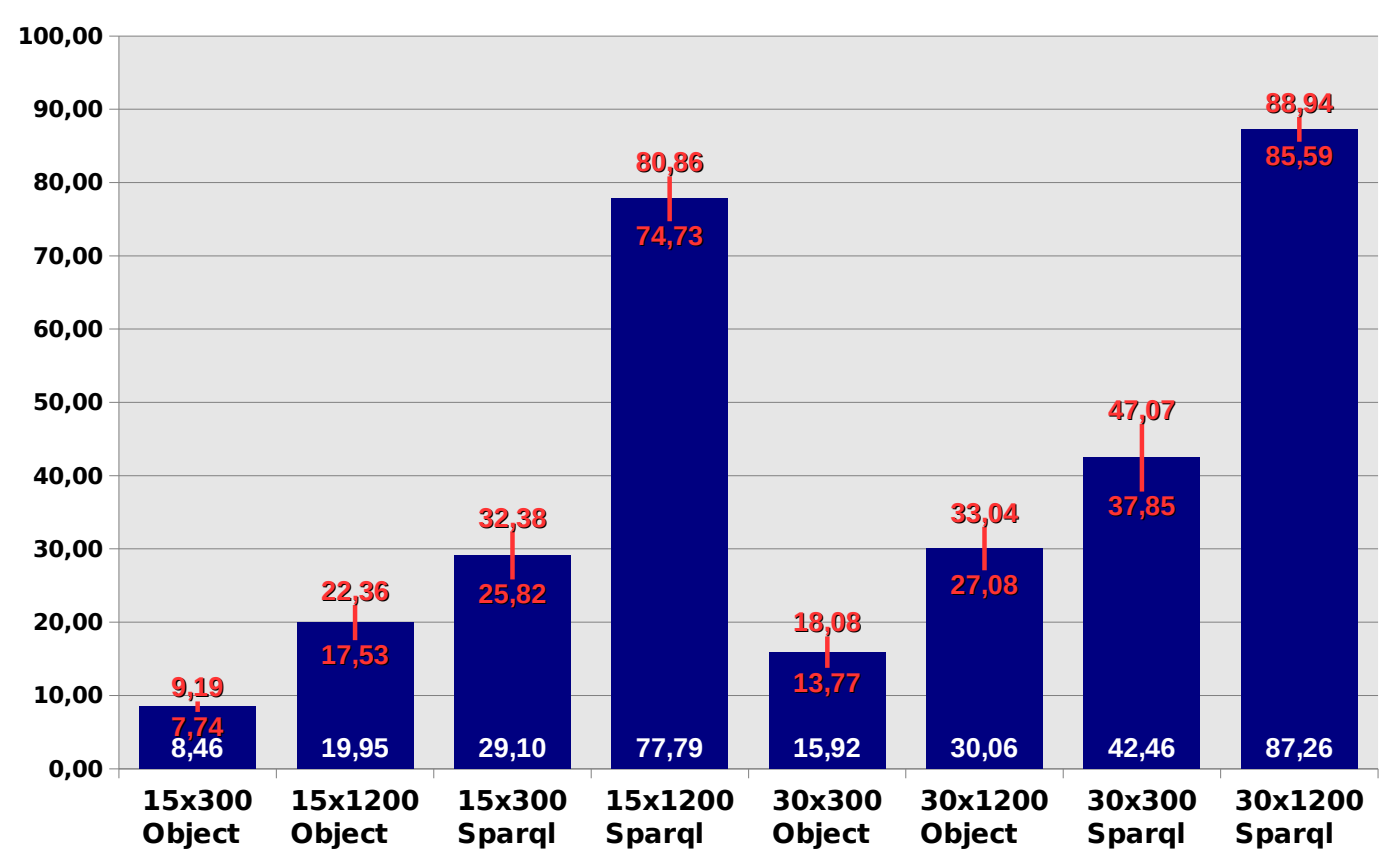

Figura 6.35: Porcentagem da carga de processamento (CPU-Load) do módulo durante os experimentos (CJE-6).

Novamente, a análise dos resultados dessa carga de processamento confirma que é preciso uma maior taxa de processamento para o algoritmo OntAlgorithmSPARQL. Quando há 1200 serviços e 30 clientes concorrentes a taxa de processamento fica próxima dos $90 \%$ de utilização dos processadores, enquanto o algoritmo OntAlgorithmObject, na pior situação, tem uma taxa de processamento por volta dos $30 \%$. Dessa forma, o OntAlgorithmObject é mais eficiente e de acordo com os resultados apresentados na Tabela 6.13 ele também possui uma melhor vazão.

\subsubsection{Conjunto de Experimentos 7}

O sétimo conjunto de experimentos (CJE-7) é semelhante ao conjunto de experimentos 5 (CJE5) com a diferença de que os fatores fixos de monitoração foram todos desativados, incluindo o fator fixo Gravação de Log (GL) que registra informações sobre o módulo, em especial informações de desempenho. Por esse motivo, não foi possível registrar o tempo de busca do módulo, sendo que os únicos registros coletados foram os tempos de resposta nos clientes (RTC).

Os resultados dos tempos de resposta para 15 clientes neste sétimo conjunto de experimentos são exibidos na Figura 6.36. 
RTC 15 Clientes - 300x600 Serviços -OntAlgorithmObject x OntAlgorithmSPARQL

口 Média (seg) I Intervalo de Confiança (seg)

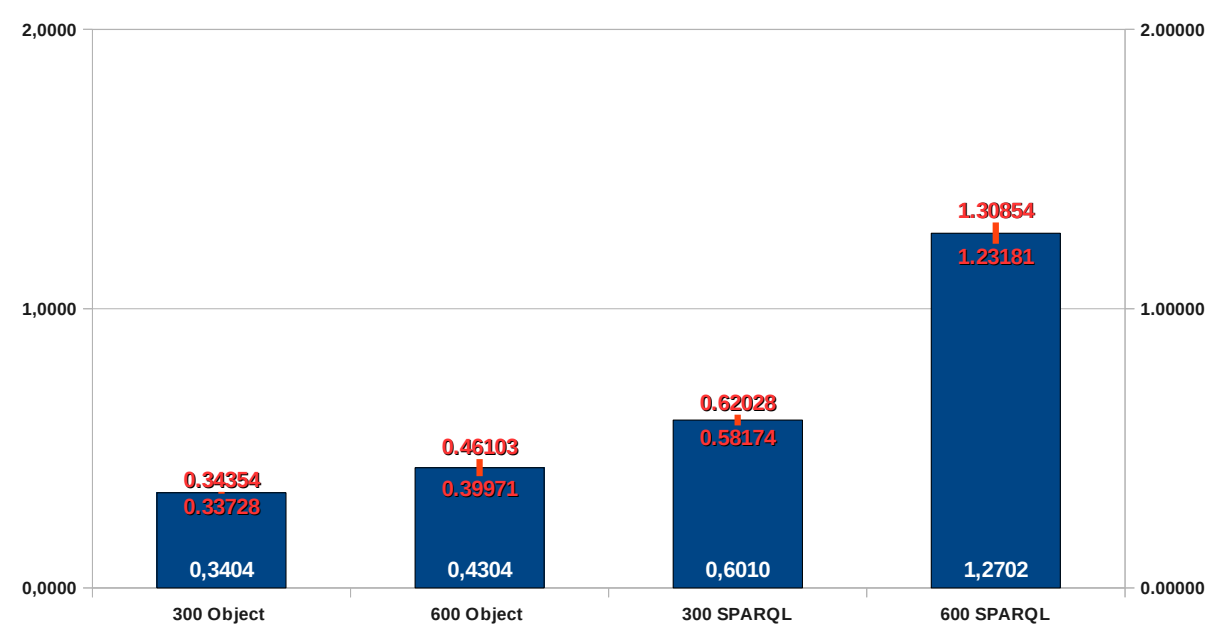

Figura 6.36: Tempo de Resposta para o Cliente (RTC) utilizando os algoritmos com 15 clientes (CJE-7).

Analisando-se os resultados da Figura 6.36 verificou-se que os experimentos que utilizam o algoritmo OntAlgorithmObject possuem um desempenho melhor. Esse comportamento é o mesmo dos outros conjuntos de experimentos e, além disso, quanto maior o número de serviços, maior é o tempo de resposta. Os resultados de tempo de resposta nos 15 clientes do CJE-7 são estatisticamente inferiores aos resultados dos outros conjuntos de experimentos correspondentes (CJE-1 e CJE-5). Esse resultado faz sentido, pois neste sétimo conjunto de experimentos não há sobrecarga imposta por nenhuma monitoração. Os resultados para 30 clientes concorrentes são exibidos na Figura 6.37 e mantêm os mesmo princípios dos resultados com 15 clientes. A diferença é que os tempos com 30 clientes são maiores.

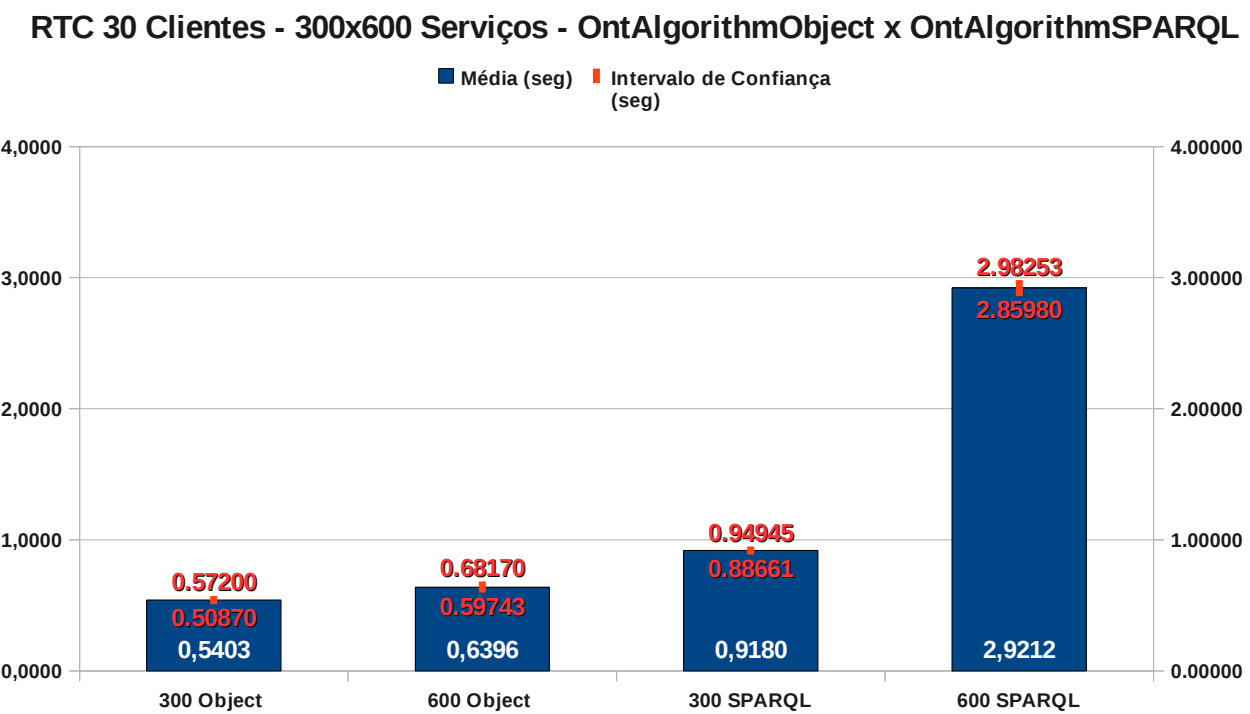

Figura 6.37: Tempo de Resposta para o Cliente (RTC) utilizando os algoritmos com 30 clientes (CJE-7). 
A Tabela 6.14 lista o fatorial completo $2^{3}$ das médias dos tempos de resposta para os clientes (RTC) e a partir dessas médias são calculadas as vazões (Throughput).

Tabela 6.14: Fatorial completo $2^{3}$ e Médias do Tempo de Busca (TB) do CJE-7

\begin{tabular}{|c|c|c|c|c|c|}
\hline Experimento & $\begin{array}{c}\text { Fator A } \\
\text { (N. Serviços) }\end{array}$ & $\begin{array}{c}\text { Fator B } \\
\text { (N. Clientes) }\end{array}$ & $\begin{array}{c}\text { Fator C } \\
\text { (Algoritmo) }\end{array}$ & RTC Média & Throughput \\
\hline 1 & 300 & 15 & Object & 0,34041 & 2,94 \\
\hline 2 & 300 & 15 & SPARQL & 0,60101 & 1,66 \\
\hline 3 & 300 & 30 & Object & 0,54035 & 1,85 \\
\hline 4 & 300 & 30 & SPARQL & 0,91803 & 1,09 \\
\hline 5 & 600 & 15 & Object & 0,43037 & 2,32 \\
\hline 6 & 600 & 15 & SPARQL & 1,27018 & 0,79 \\
\hline 7 & 600 & 30 & Object & 0,63956 & 1,56 \\
\hline 8 & 600 & 30 & SPARQL & 2,92117 & 0,34 \\
\hline
\end{tabular}

Os resultados apresentados na Tabela 6.14 facilitam a visualização da superioridade do algoritmo OntAlgorithmObject na velocidade de atendimento das requisições e, consequentemente, na vazão. As porcentagens de influência dos fatores foram calculadas e estão apresentadas na Figura 6.38 .

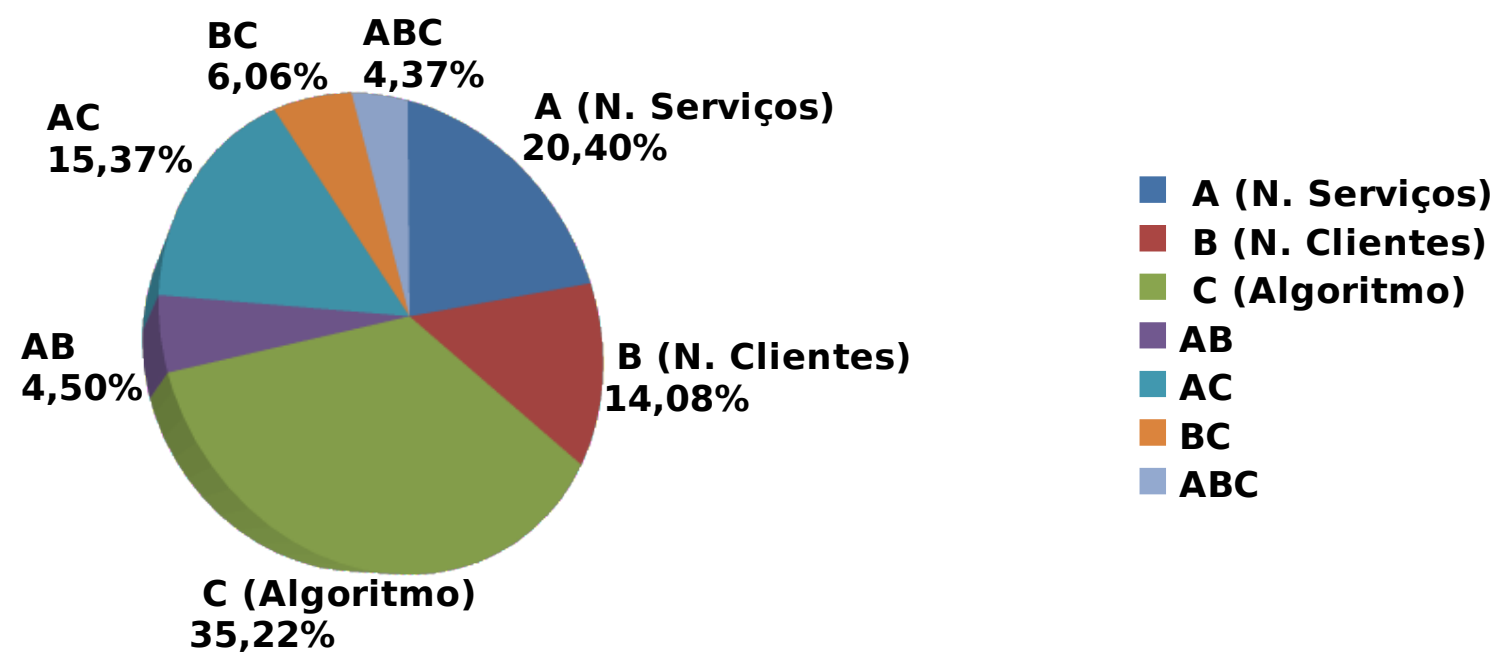

Figura 6.38: Porcentagem de Influência de cada fator no tempo de resposta nos clientes (RTC) durante os experimentos (CJE-7).

Os resultados de influência neste CJE-7 foram calculados considerando-se os tempos de respostas nos clientes. Observa-se que o fator C (Algoritmo) novamente é o fator que mais tem influência no sistema com 35,22\%. Na segunda posição ficou o fator A (Número de Serviços) com 20,40, seguido pela interação entre os fatores A e C (AC) com 15,37\%. O fator B (Número de Clientes) aparece em quarto lugar com $14,08 \%$ e as demais interações complementam o restante da influência. Nota-se que o cálculo da influência confirma que a variação de utilização de um algoritmo por outro tem impacto nos resultados. A discrepância de desempenho entre os algoritmos 
justifica essa maior influência. A quantidade de clientes concorrentes não teve muito impacto neste CJE-7. Pelo contrário, foi minimizada pela inativação dos monitoramentos e dos registradores de $\log$ e desempenho, pois, a cada requisição não foi mais preciso registrar logs em arquivos ou base de dados e nem monitorar e selecionar provedores dinamicamente. Por outro lado, não foi possível coletar dados de carga de processamento. Além disso, não é possível realizar um processo de depuração de erros, embora esses não tenham ocorrido durante os experimentos, pois todas as requisições foram atendidas.

\subsubsection{Conjunto de Experimentos 8}

O oitavo conjunto de experimentos (CJE-8) complementa o sétimo conjunto de experimentos (CJE-7). Conforme a Tabela 6.9, a diferença entre eles está apenas nos níveis do fator A (Números de Serviços) e, consequentemente, essa diferença afeta os resultados do cálculo de influência dos fatores.

Na ausência dos dados coletados pela Gravação de Log (GL) foi possível recuperar resultados apenas do Tempo de Resposta dos Clientes (RTC). Os resultados para 15 clientes concorrentes considerando 300 ou 1200 serviços com ambos os algoritmos estão exibidos em escala logarítmica na Figura 6.39.

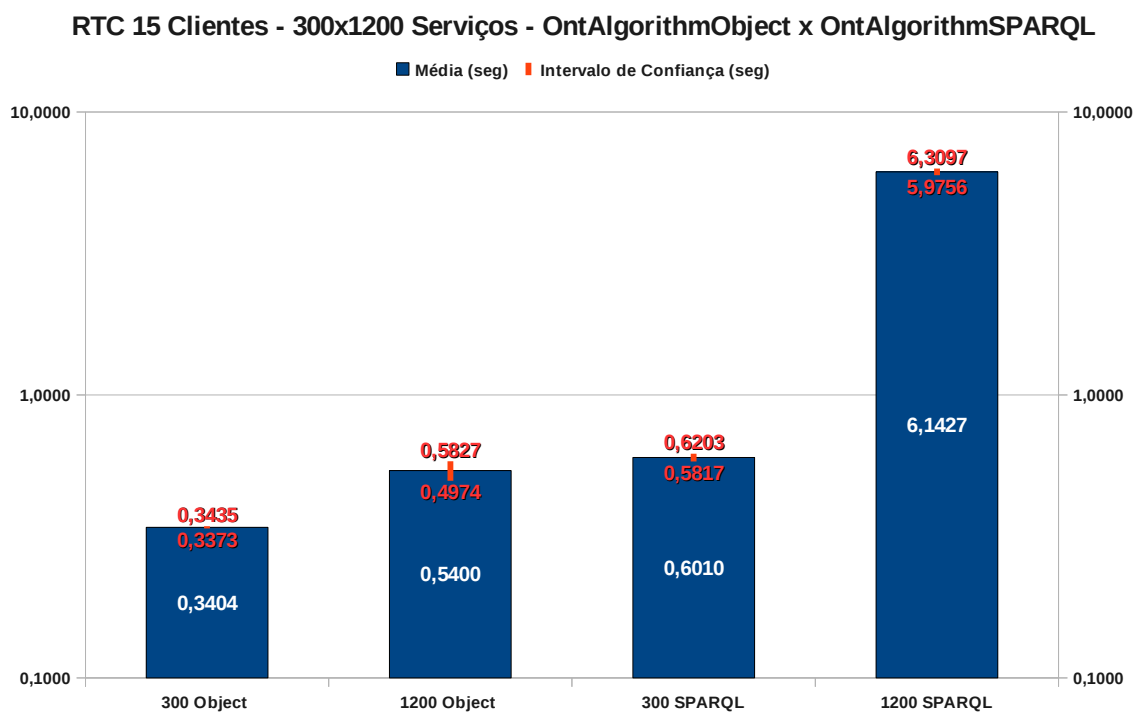

Figura 6.39: Tempo de Resposta para o Cliente (RTC) utilizando os algoritmos com 15 clientes (CJE-8).

Da mesma forma que nos outros conjuntos de experimentos, aqueles experimentos que foram executados utilizando o algoritmo OntAlgorithmObject tiveram um melhor tempo de resposta. A quantidade de número de serviços e o tempo de resposta têm uma relação direta, ou seja, quanto maior o número de serviços maior será o tempo de resposta. Contudo, esse aumento não segue um padrão linear, isso significa que dobrar ou quadruplicar o número de serviços não irá necessariamente aumentar o tempo de resposta em duas ou quatros vezes respectivamente. 
A análise dos resultados com 30 clientes é semelhante àquela com 15 clientes. Porém, os tempos de resposta são maiores devido ao aumento da carga de trabalho imposta por mais requisições concorrentes. A Figura 6.40, em escala logarítmica, exibe os resultados dos tempos de resposta do módulo com 30 clientes, 300 ou 1200 serviços e ambos os algoritmos de busca.

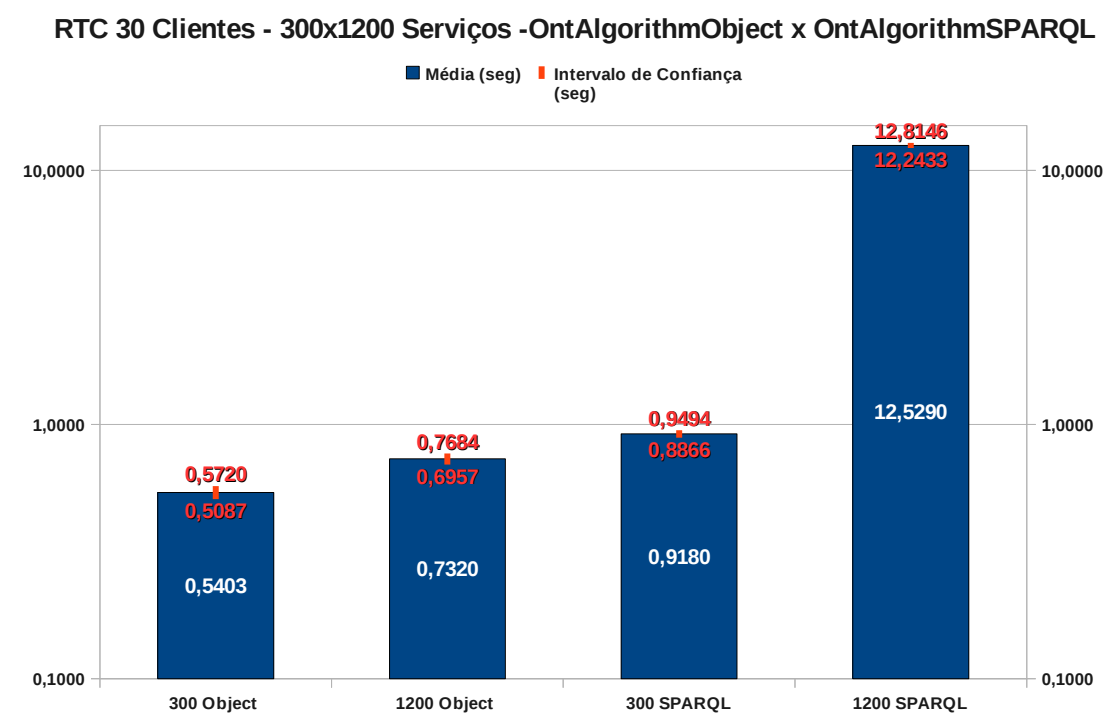

Figura 6.40: Tempo de Resposta para o Cliente (RTC) utilizando os algoritmos com 30 clientes (CJE-8).

O resultado do tempo de resposta para 30 clientes com 1200 Serviços e o algoritmo OntAlgorithmSPARQL inviabiliza a utilização do módulo nessas condições, pois, trata-se de um tempo de espera demasiadamente alto por parte do usuário (cliente). Neste oitavo conjunto de experimento não foi possível coletar informações sobre a taxa de processamento da máquina que hospeda o módulo, porém relembrando que o resultado correspondente no CJE-6 (que atingiu quase 90\% de utilização dos processadores) foi de 15,41 segundos na média, enquanto neste conjunto de experimentos (CJE-8) foi de 12,52 segundos na média. Portanto, considerando esse exemplo (o pior caso com o maior número de serviços, o maior número de clientes e o pior algoritmo) nota-se que há uma pequena influência dos fatores fixos de monitoração e de gravação de informações ( $\log s$ e desempenho). Contudo, as porcentagens de influência dos fatores fixos não puderam ser calculadas, pois embora eles variem de estado (ativo ou inativo) entre um conjunto de experimento e outro, dentro do mesmo conjunto de experimento eles estavam fixos. Variar estes fatores fixos dentro dos conjuntos de experimentos seria preciso o cálculo do fatorial completo de $2^{7}$, criando assim uma quantidade de possibilidades com 128 experimentos diferentes.

A Tabela 6.15 lista esse fatorial completo $2^{3}$ das médias dos tempos de resposta para os clientes (RTC) e com base nessas médias são calculadas as vazões (Throughput).

Com base nos resultados da Tabela 6.15 foi possível calcular a influência dos fatores que podem ser observados na Figura 6.41. 
Tabela 6.15: Fatorial completo $2^{3}$ e Médias do Tempo de Busca (TB) do CJE-8

\begin{tabular}{|c|c|c|c|c|c|}
\hline Experimento & $\begin{array}{c}\text { Fator A } \\
\text { (N. Serviços) }\end{array}$ & $\begin{array}{c}\text { Fator B } \\
\text { (N. Clientes) }\end{array}$ & $\begin{array}{c}\text { Fator C } \\
\text { (Algoritmo) }\end{array}$ & RTC Média & Throughput \\
\hline 1 & 300 & 15 & Object & 0,34041 & 2,94 \\
\hline 2 & 300 & 15 & SPARQL & 0,60101 & 1,66 \\
\hline 3 & 300 & 30 & Object & 0,54035 & 1,85 \\
\hline 4 & 300 & 30 & SPARQL & 0,91803 & 1,09 \\
\hline 5 & 1200 & 15 & Object & 0,54003 & 1,85 \\
\hline 6 & 1200 & 15 & SPARQL & 6,14269 & 0,16 \\
\hline 7 & 1200 & 30 & Object & 0,73204 & 1,37 \\
\hline 8 & 1200 & 30 & SPARQL & 12,52897 & 0,08 \\
\hline
\end{tabular}

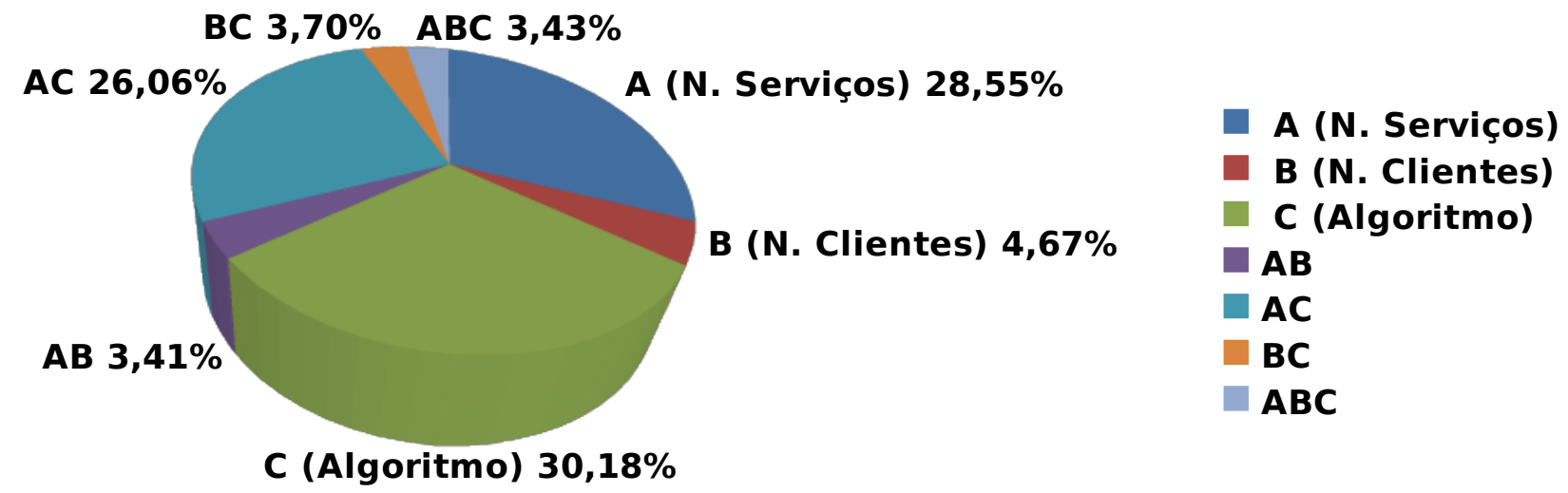

Figura 6.41: Porcentagem de Influência de cada fator no tempo de resposta nos clientes (RTC) durante os experimentos (CJE-8).

Analisando os resultados da Figura 6.41 verifica-se que, apesar de porcentagens diferentes, as posições dos principais fatores foram as mesmas da Figura 6.41. O fator C (Algoritmo) teve a maior influência no sistema com 30,18\%. Na segunda posição ficou o fator A (Número de Serviços) com $28,55 \%$, seguido pela interação entre os fatores A e C (AC) com 26,06\%. O fator B (Número de Clientes) aparece em quarto lugar com 4,67\% e as demais interações complementam o restante da influência. Nota-se que o fator A neste CJE-8 teve um aumento quando comparado ao seu correspondente no CJE-7, pois o experimento considerou um número maior de serviços cadastrados na ontologia e no registro UDDI.

\subsection{Considerações Finais}

Este capítulo abordou a avaliação de desempenho do módulo UDOnt-Q. A configuração do ambiente foi apresentada detalhando-se os recursos de software e hardware utilizados. Foram 
realizados e executados três planejamentos de experimentos, dois deles focados no processo de realização (inferência e classificação) das ontologias do módulo proposto nesta dissertação e a Pizza. O terceiro planejamento corresponde aos diversos conjuntos de experimentos que visam analisar os tempos de busca do módulo, resposta ao cliente e de vazão com relação a esse tempo de resposta. Além disso, em alguns conjuntos de experimentos foi possível identificar a sobrecarga de processamento exercida com a utilização do módulo.

Os resultados dos dois primeiros planejamentos abrem uma discussão sobre a utilização da abordagem de restrições de equivalência de classes para ontologias que possuem diversos indivíduos associados à classe ou subclasse dessas restrições, pois o tempo necessário para o processo de realização é afetado de forma não linear quando o número de indivíduos aumenta.

Com relação à análise dos resultados do terceiro planejamento, verificou-se que o fator C (Algoritmo) é o que tem mais influência no sistema. Nesse ponto, notou-se que o algoritmo OntAlgorithmObject possui melhor desempenho e eficiência que o OntAlgoritmSPARQL, devido aos motivos comentados anteriormente.

De forma geral, as conclusões sobre as análises dos resultados e propostas de melhorias serão discutidas no capitulo de Conclusões. 


\section{Conclusões}

\subsection{Conclusão Geral}

Neste projeto de mestrado diversas atividades de fundamental importância foram realizadas, antes mesmo da concepção da ontologia e desenvolvimento do módulo UDOnt-Q. Uma delas foi a pesquisa sobre metodologias de desenvolvimento de ontologias, seguida de uma aprendizagem prática nesse contexto. Outras atividades, que também demandaram pesquisa, foram o estudo de ordem teórica e prática sobre assuntos relacionados à Web Semântica.

O contexto deste trabalho está inserido em arquiteturas SOA, considerando a seleção de algumas informações de qualidade de serviço de Web Services a fim de classificá-los por meio de recursos da Web Semântica. Dessa forma, os serviços adequados podem ser selecionados, antes da busca por informações funcionais no registro UDDI. Portanto, os conhecimentos adquiridos sobre Web Semântica e Ontologia foram direcionados para a utilização prática no desenvolvimento do projeto.

O desenvolvimento da ontologia é uma das contribuições deste trabalho. Outras ontologias poderiam ter sido utilizadas, como por exemplo, a OWL-S. Mas, optou-se pela criação de uma ontologia nova, que além de acrescentar o conhecimento e aprendizado na elaboração e desenvolvimento de ontologias, pode também ser mais simples e ter um propósito especifico para atender às necessidades dos algoritmos na seleção de Web Services. Além disso, quando a elaboração do projeto estava em fase inicial, verificou-se que a OWL-S é uma ontologia complexa dividida em três níveis (camadas) e que algumas informações se repetem entre elas. Esse fato poderia prolongar o tempo de desenvolvimento dos algoritmos inviabilizando a conclusão do projeto de mestrado. 
Após a conclusão da parte de desenvolvimento, uma das maiores preocupações foi manter o ambiente de teste configurado corretamente para que os testes fossem realizados com sucesso. Foi preciso certificar-se de que os arquivos de configurações estavam corretos e que as quantidades de clientes e serviços estavam de acordo com os experimentos. Dos três algoritmos desenvolvidos, os dois escolhidos para a avaliação de desempenho foram testados exaustivamente e, assim como o módulo UDOnt-Q, provaram ser funcionais, com o algoritmo OntAlgorithmObject sendo mais eficiente em termos de tempo de busca do serviço, tempo de resposta ao cliente, vazão e eficiência de processamento. A vantagem do algoritmo OntAlgoritmSPARQL é que ele pode ser facilmente adaptado para outras ontologias, pois basicamente é preciso a alteração da query de consulta e possíveis ajustes no retorno da informação que a ontologia oferece. Porém, os resultados mostram que, nas condições propostas, esse algoritmo é o menos eficiente.

Dessa forma, este estudo produziu resultados importantes em relação a uma abordagem multidisciplinar para solucionar um problema de qualidade em um sistema distribuído, como é o caso de uma arquitetura SOA para Web Services. A execução de uma avaliação de desempenho serviu não apenas para verificar o desempenho do projeto proposto, mas também para quantificar e destacar as vantagens e desvantagens da utilização de alguns recursos da área de Web Semântica na busca por garantir a QoS adequada em Web Services.

\subsection{Contribuições}

As principais contribuições deste projeto de mestrado foram o desenvolvimento e avaliação de um módulo e seus algoritmos que fazem uso de recursos da Web Semântica na seleção de Web Services com qualidade. O módulo UDOnt-Q mostrou-se funcional e de fácil adaptação para servir de plataforma para diversos tipos de algoritmos. Além disso, o módulo disponibiliza diversos recursos de gravação de $\log s$ de erros e informações de desempenho que podem ser utilizados para diversas pesquisas que consideram a utilização de novos algoritmos na parte de seleção e composição de serviços. O módulo foi construído em componentes, o que permite que outros projetos façam uso do módulo por completo ou então utilizar apenas os seus componentes. Por exemplo, pode-se utilizar o componente UC (UDDI Components) para acesso ao registro UDDI.

Os três algoritmos desenvolvidos com recursos da Web Semântica para seleção de serviços fazem parte da contribuição deste trabalho. Os algoritmos OntAlgorithmObject e OntAlgorithmSPARQL foram submetidos a uma avaliação de desempenho e as suas vantagens e desvantagens foram destacadas. O algoritmo OntAlgorithmObject provou ser o mais eficiente e poderá ser utilizado em um ambiente de produção. Além desses algoritmos, há trabalhos de mestrado e iniciação científica em andamento no grupo de pesquisa que, ou estão utilizando, ou planejam utilizar o módulo UDOnt-Q como plataforma para seus experimentos.

A avaliação de desempenho do processo de inferência da ontologia conforme as configurações adotadas (abordagem utilizando: restrições de equivalência de classes, a máquina de inferência 
Pellet e um grande número de indivíduos) apresentou resultados interessantes, porém limitam a escalabilidade. Esses resultados indicam a necessidade de uma proposta alternativa para o processo de inferência quando se pretende trabalhar com um grande número de indivíduos ou quando esse número aumenta constantemente.

Esta dissertação de mestrado abordou tema estratégico na área de Sistemas Distribuídos, utilizando recursos de Web Semântica e Ontologia que caracterizaram, assim, um projeto multidisciplinar. O conhecimento adquirido na área de Web Semântica abre, dentro do grupo de pesquisa, um novo espaço para trabalhos que possam utilizar a Web Semântica na solução de vários problemas em sistemas distribuídos.

\subsubsection{Produção Científica}

Este projeto de mestrado permitiu a produção inicial de dois artigos que foram publicados em eventos científicos e uma monografia, publicada como Notas Didáticas, no âmbito do ICMC, como listados a seguir:

- NAKAMURA, L. H. V. ; ESTRELlA, J. C. ; SANTANA, M. J. ; SANTANA, R. H. C. Semantic Web and Ontology applied to Web Services Discovery with QoS. Proceedings of the 12th Brazilian Symposium on Computer Systems of High Performance (IEEE), 2011, Vitória, ES, Brazil.

- NAKAMURA, L. H. V. ; ESTRELlA, J. C. ; SANTANA, M. J. ; SANTANA, R. H. C. Using Semantic Web for Selection of Web Services with QoS. Proceedings of the 17th Brazilian Symposium on Multimedia and the Web, 2011. Florianópolis, SC, Brazil.

- NAKAMURA, L. H. V.; ESTRELlA, J. C.; SANTANA M. J.; SANTANA R. H. C. Desenvolvimento de Web Services com Eclipse. Notas Didáticas - ICMC-USP, 2011, São Carlos, SP.

\subsection{Trabalhos Futuros}

As contribuições alcançadas neste projeto de mestrado são significativas, pois apresentam resultados adequados do emprego de Web Semântica na seleção de Web Services com QoS. Além disso, os resultados obtidos nas avaliações de desempenho podem ser comparados com outras propostas e as aplicações desenvolvidas utilizadas em novos projetos. Em sequência ao trabalho desenvolvido, podem ser adotadas algumas melhorias para a obtenção de novos resultados. Sendo assim, para trabalhos futuros destacam-se as seguintes sugestões:

- Realizar uma análise mais detalhada nos códigos e bibliotecas do módulo, Jena e Pellet a fim de encontrar a razão do comportamento do algoritmo OntAlgorithmSPARQL. 
- Utilizar outra máquina de inferência para verificar se o comportamento no processo de inferência sofre grandes variações. Por exemplo, substituindo o Pellet pelo FaCT $++{ }^{18}$.

- O fato da máquina de inferência Pellet ocupar apenas um núcleo durante o processo de inferência leva à necessidade de mais investigação visando à solução para esse problema. Por exemplo, utilizando-se conceitos de computação concorrente (paralela) pretende-se realizar uma decomposição de dados e dividir tais dados em vários elementos de processamentos, para que sejam executados em um modelo mestre-escravo. Dessa forma, a ontologia seria replicada para cada provedor e em cada cópia da ontologia haveria apenas os indivíduos (serviços, acordos, clientes, etc.) do provedor que ela representa (decomposição de dados). Cada cópia da ontologia com seus respectivos indivíduos seriam associados a uma instância da máquina de inferência Pellet. O módulo (mestre) daria início ao processo de inferência distribuído em paralelo uma vez que cada instância do Pellet (escravo) estaria associada a um núcleo de processamento.

- Alterar o código da máquina de inferência Pellet para que utilize todos os recursos computacionais disponíveis pode ser outra solução, contudo analisando parte do código fonte do Pellet notou-se que há muitos métodos recursivos o que tornam esta tarefa não trivial.

- Novos algoritmos de seleção e composição de serviços podem ser desenvolvidos e avaliados com o auxilio do módulo UDOnt-Q.

- Novos fatores, níveis e variáveis de respostas podem ser considerados em futuras avaliações de desempenho. Por exemplo, pode-se analisar o tempo gasto pelo módulo (mais especificamente pelo componente CIS) quando os provedores precisam cadastrar as informações não funcionais (QoS) na ontologia e as funcionais no registro UDDI.

- Novas ferramentas e recursos podem ser substituídos para verificar a sua influência no desempenho. Por exemplo, substituir o SGBD MySQL pelo PostgreSQL ${ }^{19}$, o software de monitoramento distribuído Ganglia pelo $\operatorname{Nagios}^{20}$ ou ainda o framework Jena pela OWL $\mathrm{API}^{21}$.

- Outras técnicas de análise de desempenho alternativas ao processo de regressão com a análise pelo fatorial completo podem ser utilizadas. Por exemplo, pode-se utilizar futuramente a Análise de Variância com Múltiplos Fatores (ANOVA N-WAY).

- Finalmente, melhorias podem ser implementadas no módulo referente à parte de segurança e tolerância a falhas.

\footnotetext{
${ }^{18}$ http://owl.man.ac.uk/factplusplus/

${ }^{19} \mathrm{http}: / / \mathrm{www} \cdot$ postgresql.org/

${ }^{20} \mathrm{http}$ //www.nagios.org/

${ }^{21} \mathrm{http}$ ://owlapi.sourceforge.net/
} 


\section{Referências}

AkLOUF, Y.; REZIG, E. An ontological approach for dynamic functionality-based web services discovery using expert systems. In: Applications of Digital Information and Web Technologies, 2009. ICADIWT '09. Second International Conference on the, 2009, p. 187 -192.

AMBLER, S. Agile modeling: Effective practices for extreme programming and the unified process. New York, NY, USA: John Wiley \& Sons, Inc., 2002.

Antoniou, G.; Harmelen, F. V. Semantic web primer, 2nd edition. Mit Press, 2008.

Baocai, Y.; Huirong, Y.; Pengbin, F.; Liheng, G.; Mingli, L. A framework and qos based web services discovery. In: Software Engineering and Service Sciences (ICSESS), 2010 IEEE International Conference on, 2010, p. 755 -758.

Bernaras, A.; LAResgoiti, I.; Corera, J. Building and reusing ontologies for electrical network applications. Proceedings of the European Conference on Artificial Intelligence (ECAI'96), p. 298-302, 1996.

Berners-Lee, T. Semantic web on xml - semantic web - xml2000. Disponível em: http: / / www .w3 .org/2000/Talks/1206-xml2k-tbl/. Último acesso: 14/12/2009, 2000.

Bhakti, M.; Abdullah, A. Design of an autonomic services oriented architecture. In: Information Technology (ITSim), 2010 International Symposium, 2010, p. 805 -810.

Broekstra, J.; Klein, M.; Decker, S.; Fensel, D.; Harmelen, F.; Horrocks, I. Enabling knowledge representation on the web by extending rdf schema. In: In Proceedings of the Tenth International World Wide Web Conference (WWW10), Hong Kong, 2001.

CARASE, S. J. Uma ontologia funcional de reputação para agentes. Dissertação de mestrado em engenharia elétrica, Escola Politécnica da Universidade de São Paulo - USP, São Paulo, SP, 2005. 
Carroll, J. J.; Dickinson, I.; Dollin, C.; Reynolds, D.; Seaborne, A.; Wilkinson, $\mathrm{K}$. Jena: implementing the semantic web recommendations. In.Proceedings of the 13th international World Wide Web conference on Alternate track papers and posters, 2004.

Endrei, M.; Ang, J.; Arsanjani, A.; Chua, S.; Comte, P.; Krogdahl, P.; Luo, M.; Newling, T. Patterns: Service-oriented architecture and web services. IBM Redbooks, disponível em: http: / / www.redbooks.ibm.com/redbooks/pdfs / sg2 46303 . pdf. Último acesso: 15/12/2009, 2004.

ERL, T. Soa princípios de design de serviços. Pearson Prentice Hall PTR, 2009.

ERRADI, A.; MAHEShWARI, O. A broker-based approach for improving web services reliability. In: IEEE International Conference on Web Services (ICWS'05), 2005.

Estrella, J.; Toyohara, R.; Kuehne, B.; Tavares, T.; Santana, R.; Santana, M.; BRUSCHI, S. A performance evaluation for a qos-aware service oriented architecture. In: Services (SERVICES-1), 2010 6th World Congress on, 2010, p. 260 -267.

ESTRELla, J. C. Wsarch: Uma arquitetura para provisão de web services com qualidade de serviço. Tese de doutorado, ICMC-USP, São Carlos, SP, 2010.

FAKhfakh, K.; ChaAri, T.; TAZI, S.; DriRA, K.; JMAiel, M. A comprehensive ontologybased approach for sla obligations monitoring. The Second International Conference on Advanced Engineering Computing and Applications in Sciences, 2008.

FARKas, P.; ChARAF, H. Web services planning concepts. Journal Of .net Technologies, p. 9-12, 2003.

Fisher, M.; Blace, R.; Hebeler, J.; Perez-Lopez, A. Semantic web programming. Wiley, 2009.

Gennari, J. H.; Musen, M. A.; Fergerson, R. W.; Grosso, W. E.; Crubzy, M.; EriksSON, H.; NOY, N. F.; TU, S. W. The evolution of protégé: An environment for knowledgebased systems development. International Journal of Human-Computer Studies, 2002.

Goh, C. M.; LEE, S. P.; He, W.; TAN, P. S. Web 2.0 concepts and technologies for dynamic b2b integration. In: Emerging Technologies and Factory Automation - ETFA, IEEE, 2007.

Gruninger, M.; Fox, M. S. Methodology for design and evaluation of ontologies. In: Proceedings of Workshop on basic Ontological Issues in Knowledge Sharing, 1995.

Heflin, J. D. Towards the semantic web: Knowledge representation in a dynamic, distributed environment. Tese de doutorado, University of Maryland, Maryland - USA, 2001. 
Holger, K.; Rector, A.; Stevens, R.; Wroe, C.; Jupp, S.; Moulton, G.; Drummond, N. A Practical Guide To Building OWL Ontologies Using Protégé 4 and CO-ODE Tools Edition 1.2. The University Of Manchester, disponível em: http://owl.cs.manchester.ac.uk/tutorials/protegeowltutorial/ resources/ProtegeoWLTutorialP4_v1_2.pdf, 2009.

HopPer, J. Reduza o consumo de energia do linux, parte 1: Subsistema cpufreq. Disponível em: http://www.ibm.com/developerworks/br/linux/library/l-cpufreq-1/. Último acesso: 14/09/2011, 2009.

IBM Web service level agreements (wsla) project. Disponível em: http: / / www . research . ibm. com/wsla/about.html. Último acesso: 10/12/2009, 2009.

Isotani, S.; Mizoguchi, R.; Bittencourt, I. I.; Costa, E. D. B. Estado da arte em web semântica e web 2.0: Potencialidades e tendências da nova geração de ambientes de ensino na internet. Revista Brasileira de Informática na Educação, v. 17, p. 30-42, 2009.

JAIN, R. The art of computer systems performance analysis: techniques for experimental design, measurement, simulation, and modeling. Wiley, 1991.

Josuttis, N. M. SOA na prática - A Arte da Modelagem de Sistemas Distribuídos. 1st. ed. Oreill'y, 2008.

Komoda, N. Service oriented architecture (soa) in industrial systems. In: IEEE International Conference on Industrial Informatics, IEEE, 2006.

Kritikos, K.; Plexousakis, D. Requirements for qos-based web service description and discovery. In: Computer Software and Applications Conference, 2007. COMPSAC 2007. 31st Annual International, 2007, p. 467 -472.

LeE, S.; ShIN, D. Web service qos in multi-domain. In: Proceedings of Advanced Communication Technology, - ICACT, 2008.

LeE, T. B.; Fischetti, M. Weaving the web - the original design and ultimate destiny of the world wide web, by its inventor. ISBN 006251587X, 1997.

LEE, Y. Quality-context based soa registry classification for quality of services. In: Proceedings of the 2008 IEEE International WorkShop on Semantic Computing and Applications, IEEE, 2008.

Lopez, M.; Gomez-Perez, A.; Sierra, J.; Sierra, A. Building a chemical ontology using methontology and the ontology design environment. Intelligent Systems and their Applications, IEEE, v. 14 , n. 1 , p. $37-46,1999$. 
Lopez, M. F.; GomeZ-Perez, A.; Juristo, N. METHONTOLOGY: from Ontological Art towards Ontological Engineering. In: Proceedings of the AAAI97 Spring Symposium, Stanford, USA, 1997, p. 33-40.

Luo, J.; Montrose, B.; Kim, A.; Khashnobish, A.; Kang, M. Adding owl-s support to the existing uddi infrastructure. In: IEEE International Conference on Web Services (ICWS'O6), IEEE, 2006.

MA, C.; SONG, M.; XU, K.; ZHANG, X. Web service discovery research and implementation based on semantic search engine. In: Web Society (SWS), 2010 IEEE 2nd Symposium on, 2010, p. $672-677$.

Malik, S.; Goel, A.; Maniktala, S. A comparative study of various variants of sparql in semantic web. In: Computer Information Systems and Industrial Management Applications (CISIM), 2010 International Conference on, 2010, p. 471 -474.

Martimiano, L. A. F. Sobre a estruturação de informação em sistemas de segurança computacional: o uso de ontologia. Tese de doutorado em ciências de computação e matemática computacional, ICMC-USP, São Carlos, SP, 2006.

Martin, D.; Burstein, M.; Hobbs, J.; Lassila, O.; McDermott, D.; McIlraith, S.; Narayanan, S.; Paolucci, M.; Parsia, B.; Payne, T.; Sirin, E.; SriniVASAN, N.; SYCARA, K. Owl-s: Semantic markup for web services. Disponível em: http://www.w3.org/Submission/OWL-S/. Último acesso: 14/08/2009, 2004.

Massie, M. L.; Chun, B. N.; Culler, D. E. The ganglia distributed monitoring system: Design, implementation and experience. Parallel Computing, v. 30, p. 2004, 2003.

Matos, J. Distribuição de carga flexível e dinâmica para provedores de web services. Dissertação de mestrado, ICMC - Universidade de São Paulo, São Carlos, SP, 2009.

Maximilien, E. M.; Singh, M. P. A framework and ontology for dynamic web services selection. IEEE Internet Computing, 2004.

Murugesan, S. Understanding web 2.0. IT Professional, v. 9, n. 4, p. 34-41, 2007.

Nakamura, L. V.; Estrella, J. C.; Santana, M. J.; Santana, R. H. C. Semantic web and ontology applied to web services discovery with qos. Proceedings of the 12th Brazilian Symposium on Computer Systems of High Performance (IEEE), 2011, Vitória, ES, Brasil., 2011a.

Nakamura, L. V.; Estrella, J. C.; Santana, M. J.; Santana, R. H. C. Using semantic web for selection of web services with qos. Proceedings of the 17th Brazilian Symposium on Multimedia and the Web, Florianópolis, SC, Brazil, 2011 b. 
Noy, N. F.; McGuinness, D. L. Ontology development 101: A guide to create your first ontology. Relatório Técnico, Knowledge System Laboratory - Stanford University, 2001, 2001.

Oren, E.; Kotoulas, S.; Anadiotis, G.; Siebes, R.; Teije, A.; Harmelen, F. Marvin: Distributed reasoning over large-scale semantic web data. Web Semant., v. 7, p. 305-316, 2009.

Papaionnnou, I. V.; Tsesmetzis, D. T.; Roussaki, I. G.; Anagnostou, M. E. A qos ontology language for web-services. Proceedings of the 20th International Conference on Advanced Information Networking and Applications (AINA'06), 2006.

Papazoglou, M. P.; Georgakopoulos, D. Service-oriented computing. Communications of the ACM. October 2003/Vol. 46, No. 10, 2003.

Prud'hommeaux, E.; Seaborne, A. Sparql query language for rdf. Disponível em: http://www.w3.org/TR/rdf-sparql-query/. Último acesso: 05/03/2011, 2008.

RAJEndRAn, T.; BAlasubramanie, P. An optimal agent-based architecture for dynamic web service discovery with qos. In: Computing Communication and Networking Technologies (ICCCNT), 2010 International Conference on, 2010, p. 1 -7.

SEABORne, A. RDQL - A Query Language for RDF. Disponível em: http://www.w3.org/Submission/RDQL/ Último acesso: 19/04/2009, 2004a.

SEABORne, A. A programmer's introduction to rdql. Disponível em: http://jena.sourceforge.net/tutorial/RDQL/ Último acesso: 19/04/2009, 2004 b.

SIRIN, E.; PARSIA, B. Sparql-dl: Sparql query for owl-dl. In: In 3rd OWL Experiences and Directions Workshop (OWLED-2007, 2007.

Sirin, E.; Parsia, B.; Grau, B. C.; KalyanPur, A.; Katz, Y. Pellet: A practical owl-dl reasoner. ACM - Journal of Web Semantics, 2007.

Smith, M. K.; Welty, C.; McGuinness, D. L. Owl web ontology language- guide. Disponível em: http://www.w3.org/TR/owl-guide/. Último acesso: 14/08/2009, 2004.

Tanenbaum, A. S. Redes de computadores. 4th. ed. Editora Campus, 2003.

TAVARES, R. K.; Westphall, C. B. An architecture to provide qos in web services. In: Communications Society subject matter experts for publication in the IEEE ICC 2006 proceedings, IEEE Computer Society, 2006.

TAVARES, T. C. Caracterização de cargas de trabalho para avaliação de desempenho em web services. Dissertação de mestrado, Universidade de São Paulo, São Carlos, SP, 2009.

Thomas, J. P.; Thomas, M.; Ghinea, G. Modeling of web services flow. In: Proceedings of the IEEE International Conference on E-Commerce (CEC'03), IEEE Computer Society, 2003. 
TOYOHARA, R. K. T. Construindo web services para avaliação de desempenho de uma arquitetura orientada a serviços com qos. Qualificação de mestrado, USP - Universidade de São Paulo, São Carlos, SP, 2009.

Tran, V. X.; Tsuji, H. A survey and analysis on semantic in qos for web services. In.Proceedings of the international Conference on Advanced Information Networking and Applications, 2009.

Tran, X. V. Ws-qosonto: A qos ontology for web services. In: In: 2008 IEEE International Symposium on Service-Oriented System Engineering, IEEE International Symposium, 2008.

TSAI, Y.-H.; HWANG, S.-Y.; TANG, Y. A hybrid approach to automatic web services discovery. In: Service Sciences (IJCSS), 2011 International Joint Conference on, 2011, p. 277 -281.

UsChOld, M.; GRUninger, M. Ontologies: principles, methods and applications. Knowledge Engineering Review, 93-155 p., 1996.

W3C Owl web ontology language reference. Disponível em: http://www.w3.org/TR/owl-ref/ Último acesso: 21/04/2009, 2004a.

W3C Resource description framework (rdf): Concepts and abstract syntax. Disponível em: http://www.w3.org/TR/2004/REC-rdf-concepts-20040210/ Último acesso: 21/04/2009, 2004b.

W3C Web ontology language overview. Disponível em: http://www.w3.org/TR/owl-features/ Último acesso: 21/04/2009, 2004c.

W3C Web services architecture. Disponível em: http://www.w3.org/TR/2004/NOTE-ws-arch20040211/ Último acesso: 19/04/2009, 2004d.

Wu, H.; GuO, C. The research and implementation of web service classification and discovery based on semantic. In: Computer Supported Cooperative Work in Design (CSCWD), 2011 15th International Conference on, 2011, p. 381 -385.

Xu, Z.; Martin, P.; Powley, W.; Zulkernine, F. Reputation-enhanced qos-based web services discovery. Proceedings of the 2007 IEEE International Conference on Web Services (ICWS), 2007.

Ye, G.; Wu, C.; Yue, J.; Cheng, S. A qos-aware model for web services discovery. Proceedings of the 2009 First International Workshop on Education Technology and Computer Science, 2009.

YUAN-SHENG, L.; XIAO, H.; YU, W.; SI-XIN, S. Research on a web services discovery model framework. In: Computational Intelligence and Software Engineering (CiSE), 2010 International Conference on, 2010, p. 1 -4. 
Zhou, C.; ChiA, L.; LeE, B. Qos measurement issues with daml-qos ontology. Proceedings of the 2005 IEEE International Conference on e-Business Engineering (ICEBE'05), 2005. 
ANEXOS 



\section{ANEXO 1}

\section{Documentos de Especificações da Ontologia}

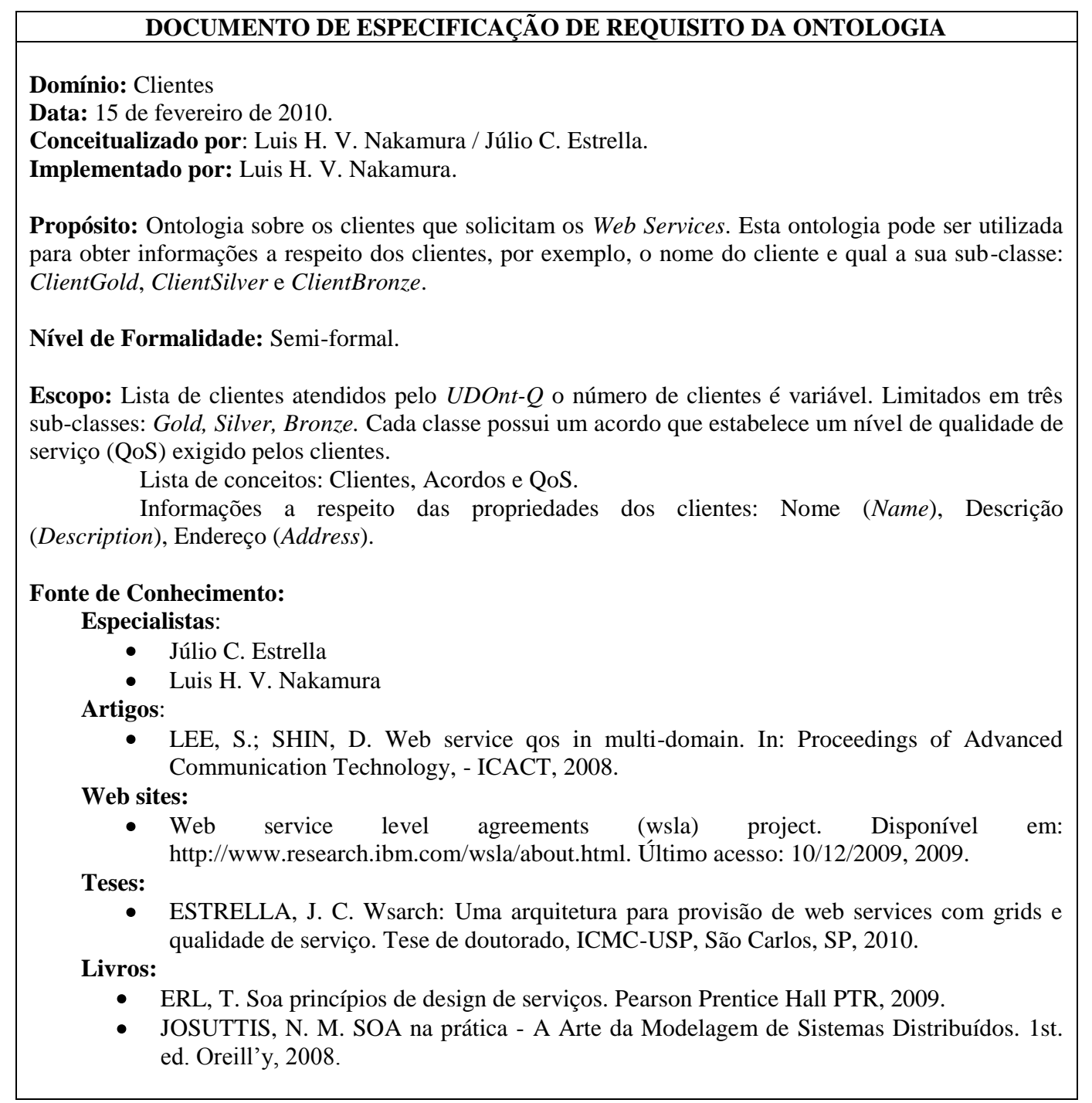




\section{DOCUMENTO DE ESPECIFICAÇÃO DE REQUISITO DA ONTOLOGIA}

Domínio: Acordo

Data: 15 de fevereiro de 2010.

Conceitualizado por: Luis H. V. Nakamura / Júlio C. Estrella.

Implementado por: Luis H. V. Nakamura.

Propósito: Ontologia sobre os acordos existentes que são definidos nas classes dos clientes, os quais solicitam os Web Services. Esta ontologia pode ser utilizada para obter informações a respeito dos acordos, por exemplo, tipo do acordo (Light, Medium, Heavy).

Nível de Formalidade: Semi-formal.

Escopo: Lista de acordos que podem estar estabelecidos nas classes dos clientes. Cada classe tem um acordo relacionado, cada acordo tem uma sub-classe (LightAgreement, MediumAgreement e HeavyAgreement) estabelece relação com $Q o S$ a qual contém os níveis (valores) dos parâmetros de qualidade de serviço (QoS).

Lista de conceitos: Acordos (Light, Medium, Heavy), parâmetros e valores de QoS.

Informações a respeito das propriedades dos acordos: Nome (Name) e Descrição (Description).

Fonte de Conhecimento:

Especialistas:

- Júlio C. Estrella

- Luis H. V. Nakamura

Artigos:

- LEE, S.; SHIN, D. Web service qos in multi-domain. In: Proceedings of Advanced Communication Technology, - ICACT, 2008.

Web sites:

- Web service level agreements (wsla) project. Disponível em: http://www.research.ibm.com/wsla/about.html. Último acesso: 10/12/2009, 2009.

\section{Livros:}

- $\quad$ ERL, T. Soa princípios de design de serviços. Pearson Prentice Hall PTR, 2009.

- JOSUTTIS, N. M. SOA na prática - A Arte da Modelagem de Sistemas Distribuídos. 1st. ed. Oreill'y, 2008. 


\section{DOCUMENTO DE ESPECIFICAÇÃO DE REQUISITO DA ONTOLOGIA}

Domínio: Qualidade de Serviço (QoS)

Data: 15 de fevereiro de 2010.

Conceitualizado por: Luis H. V. Nakamura / Júlio C. Estrella.

Implementado por: Luis H. V. Nakamura.

Propósito: Ontologia sobre a qualidade de serviço (QoS) dos Web Services que são solicitados pelos clientes. Esta ontologia pode ser utilizada para obter informações a respeito da qualidade de serviço dos Web Services, por exemplo, a disponibilidade de utilização de um determinado Web Service.

Nível de Formalidade: Semi-formal.

Escopo: Lista de parâmetros de qualidade de serviços que podem ser prestados em um Web Service pelos provedores, número fixo de 16 parâmetros ${ }^{1}$.

Lista de conceitos: Qualidade de serviço, Parâmetros (atributos ou propriedades), Níveis (ou valores), sub-classes: QoSGold, QoSSilver e QoSBronze. O que determina a qual sub-classe uma QoS pertence são as restrições de classes equivalentes criadas na ontologia e os níveis (valores) que um atributo de $Q o S$ possui. Por exemplo, caso a restrição de classe equivalente indique que:

- Todas as $Q o S$ que tenham o atributo Response_Time (tempo de resposta) menor que 1 (um) segundo serão equivalentes a classe QoSGold.

- Todas as $Q o S$ que tenham o atributo Response_Time (tempo de resposta) entre 1 e 3 segundos serão equivalentes a classe QoSSilver.

- Todas as $Q o S$ que tenham o atributo Response_Time (tempo de resposta) maior que 3 segundos serão equivalentes a classe QoSBronze.

Isso implique que toda classe $Q o S$ que tenha o atributo Response_Time com um determinado valor (nível) será classificado em uma das três sub-classes quando a ontologia for inferida.

Fonte de Conhecimento:

Especialistas:

- Júlio C. Estrella

- Luis H. V. Nakamura

Artigos / Documentos:

- LEE, S.; SHIN, D. Web service qos in multi-domain. In: Proceedings of Advanced Communication Technology, - ICACT, 2008.

- Holger, K., Rector, A., Stevens, R., Wroe, C., Jupp, S., Moulton, G., and Drummond, N. (2009). A Practical Guide To Building OWL Ontologies Using Protégé 4 and CO-ODE Tools Edition 1.2.

Web sites:

- Web service level agreements (wsla) project. Disponível em: http://www.research.ibm.com/wsla/about.html. Último acesso: 10/12/2009, 2009.

\footnotetext{
${ }^{1}$ Parâmetros: Tempo de resposta, Latência, Vazão, Escalabilidade, Disponibilidade, Confiabilidade, Acessibilidade, Capacidade, Precisão, Robustez, Estabilidade, Custo, Segurança, Integridade, Desempenho e Interoperabilidade.
} 


\section{DOCUMENTO DE ESPECIFICAÇÃO DE REQUISITO DA ONTOLOGIA}

Domínio: Serviços

Data: 15 de fevereiro de 2010.

Conceitualizado por: Luis H. V. Nakamura / Júlio C. Estrella.

Implementado por: Luis H. V. Nakamura.

Propósito: Ontologia sobre os Web Services que são solicitados pelos clientes. Esta ontologia pode ser utilizada para obter informações a respeito dos serviços, por exemplo, o nome do serviço.

Nível de Formalidade: Semi-formal.

Escopo: Lista de serviços prestados pelos provedores é um número variável.

Lista de conceitos: Web services, QoS, WSDL.

Informações a respeito das propriedades dos serviços: Nome (Name), Descrição (Description), Endereço (Address), Empresa/Instituição responsável (Company), principal Desenvolvedor responsável (Developer), interface WSDL (Interface).

Fonte de Conhecimento:

Especialistas:

- Júlio C. Estrella

- Luis H. V. Nakamura

Artigos:

- LEE, S.; SHIN, D. Web service qos in multi-domain. In: Proceedings of Advanced Communication Technology, - ICACT, 2008.

Web sites:

- Web service level agreements (wsla) project. Disponível em: http://www.research.ibm.com/wsla/about.html. Último acesso: 10/12/2009, 2009.

Teses:

- ESTRELLA, J. C. Wsarch: Uma arquitetura para provisão de web services com grids e qualidade de serviço. Tese de doutorado, ICMC-USP, São Carlos, SP, 2010.

Livros:

- $\quad$ ERL, T. Soa princípios de design de serviços. Pearson Prentice Hall PTR, 2009.

- JOSUTTIS, N. M. SOA na prática - A Arte da Modelagem de Sistemas Distribuídos. 1st. ed. Oreill'y, 2008. 


\section{DOCUMENTO DE ESPECIFICAÇÃO DE REQUISITO DA ONTOLOGIA}

Domínio: Provedores

Data: 15 de fevereiro de 2010.

Conceitualizado por: Luis H. V. Nakamura / Júlio C. Estrella.

Implementado por: Luis H. V. Nakamura.

Propósito: Ontologia sobre os provedores de Web Services que atendem as requisições dos clientes. Esta ontologia pode ser utilizada para obter informações a respeito dos provedores de serviços, por exemplo, nome do provedor.

Nível de Formalidade: Semi-formal.

Escopo: Lista de provedores dentro da arquitetura, o número de provedores é variável. A relação de cardinalidade entre os provedores e os serviços é de zero para muitos. Lista de conceitos: Provedor, Serviço.

Informações a respeito das propriedades dos provedores: Nome (Name), Endereço (Address), Empresa/Instituição responsável (Company).

Fonte de Conhecimento:

\section{Especialistas:}

- Júlio C. Estrella

- Luis H. V. Nakamura

\section{Artigos:}

- LEE, S.; SHIN, D. Web service qos in multi-domain. In: Proceedings of Advanced Communication Technology, - ICACT, 2008.

Web sites:

- Web service level agreements (wsla) project. Disponível em: http://www.research.ibm.com/wsla/about.html. Último acesso: 10/12/2009, 2009.

Teses:

- ESTRELLA, J. C. Wsarch: Uma arquitetura para provisão de web services com grids e qualidade de serviço. Tese de doutorado, ICMC-USP, São Carlos, SP, 2010.

Livros:

- $\quad$ ERL, T. Soa princípios de design de serviços. Pearson Prentice Hall PTR, 2009.

- JOSUTTIS, N. M. SOA na prática - A Arte da Modelagem de Sistemas Distribuídos. 1st. ed. Oreill'y, 2008. 


\section{ANEXO 2}

\section{Documentos da Conceituação da Ontologia Glossário de Termos - GT (Glossary of Terms)}

\begin{tabular}{|c|c|}
\hline Nome & Descrição \\
\hline $\begin{array}{l}\text { Agreement } \\
\text { (Acordo) }\end{array}$ & $\begin{array}{l}\text { Acordo estabelecido entre o consumidor do serviço (cliente) e o provedor de } \\
\text { serviço a respeito da Qualidade de Serviço (QoS) prestada. Cada acordo } \\
\text { possui uma subclasse (Agreement[SubClass]). O que determina a subclasse } \\
\text { do Acordo é a subclasse de QoS relacionada a ele. }\end{array}$ \\
\hline $\begin{array}{l}\text { [SubClass }] \text { Agree } \\
\text { ment (Sub- } \\
\text { Classes do } \\
\text { Acordo) }\end{array}$ & $\begin{array}{l}\text { O acordo (Agreement) pode ter sub-classes são os tipos que um acordo pode } \\
\text { assumir. No escopo desta ontologia existem três sub-classes de acordos: 1) } \\
\text { LightAgreement, 2) MediumAgreement e } 3 \text { ) HeavyAgreement, que que } \\
\text { determinam o nível de rigorosidade do acordo. Sendo o Light aqueles que } \\
\text { possuem parâmetros de QoS com valores menos rigorosos, o Heavy com } \\
\text { valores mais rigorosos e o Medium é um meio termo. A ontologia deve ser } \\
\text { extensível para aceitar novas sub-classes de Acordos. }\end{array}$ \\
\hline $\begin{array}{l}\text { Client (Cliente ou } \\
\text { Consumidor do } \\
\text { Serviço) }\end{array}$ & $\begin{array}{l}\text { O cliente ou consumidor pode tanto ser um usuário quanto um agente } \\
\text { computacional de uma empresa. (EAI, B2C e B2B). O cliente deve estar } \\
\text { relacionado a um único Acordo. }\end{array}$ \\
\hline $\begin{array}{l}\text { Client }[\text { SubClass }] \\
\text { (Sub-Classe de } \\
\text { Clientes) }\end{array}$ & $\begin{array}{l}\text { Os clientes são subdividos em classes (grupos), cada classe tem um tipo e um } \\
\text { acordo relacionado. Dependendo do tipo de acordo o cliente pode ser um } \\
\text { ClientGold (HeavyAgreement), ClientSilver (MediumAgreement) ou } \\
\text { ClientBronze (LightAgreement). }\end{array}$ \\
\hline $\begin{array}{l}\text { Provider } \\
\text { (Provedores ou } \\
\text { Servidores) }\end{array}$ & $\begin{array}{l}\text { Os provedores (servidores) de serviços são (geralmente) empresas que } \\
\text { prestam serviços web (Web Services). A utilização desses serviços poderá ou } \\
\text { não envolver custos financeiros. Os provedores são responsáveis por publicar } \\
\text { os serviços disponíveis no registro UDDI e quando existir um acordo deve } \\
\text { cumpri-lo caso contrário poderá haver a penalidade de multa. Os serviços } \\
\text { que possuem parâmetros e níveis de QoS são monitorados constantemente } \\
\text { pelos provedores que ficam responsáveis pode garantir a qualidade de } \\
\text { serviço e manter as bases de informações (UDDI e Ontologia) atualizadas } \\
\text { quanto a estas informações. A escolha final do serviço quando existirem } \\
\text { mais de um serviço que atenda as requisições de QoS pode ser determinada } \\
\text { por agentes externos de monitoramento, portanto informações relevantes, } \\
\text { como por exemplo, o endereço do provedor, devem está presentes na } \\
\text { ontologia. }\end{array}$ \\
\hline $\begin{array}{c}\text { Service (Web } \\
\text { Service) }\end{array}$ & $\begin{array}{l}\text { Os Web Services são prestados pelos provedores e consumidos pelos } \\
\text { clientes. A lista de serviços se encontra no registro UDDI. Os serviços estão } \\
\text { associados à classe QoS, ou seja os serviços têm QoS (parâmetros de QoS). }\end{array}$ \\
\hline $\begin{array}{l}\text { Service }[\text { Class }] \\
\text { (Sub-Classe de } \\
\text { Web Service) }\end{array}$ & $\begin{array}{l}\text { Assim como os Acordos, os Web Services são subdividos em três subclasses } \\
\text { (ServiceGold, ServiceSilver e ServiceBronze), a classi- ficação em cada uma } \\
\text { dessas subclasses depende do seu relacionamento com a subclasse de QoS. } \\
\text { Por exemplo, QoSGold infere em um ServiceGold, QoSSilver infere em um } \\
\text { ServiceSilver e QoSBronze infere em um ServiceBronze. }\end{array}$ \\
\hline$Q o S$ & $\begin{array}{l}\text { QoS - Qualidade de Serviço, a qualidade de serviço é composta por } \\
\text { parâmetros (atributos). Cada parâmetro tem um nível (valor) e um tipo de } \\
\text { dado. São os serviços (Web Services) e o Acordos que dispõem de QoS. Os } \\
\text { atributos de QoS serão aqueles especificados na Tabela de QoS (Lee e Shin, } \\
\text { 2008). }\end{array}$ \\
\hline
\end{tabular}




\section{$\underline{\text { Árvore de Classificacão de Conceitos (Concept-Classification Tree) }}$}

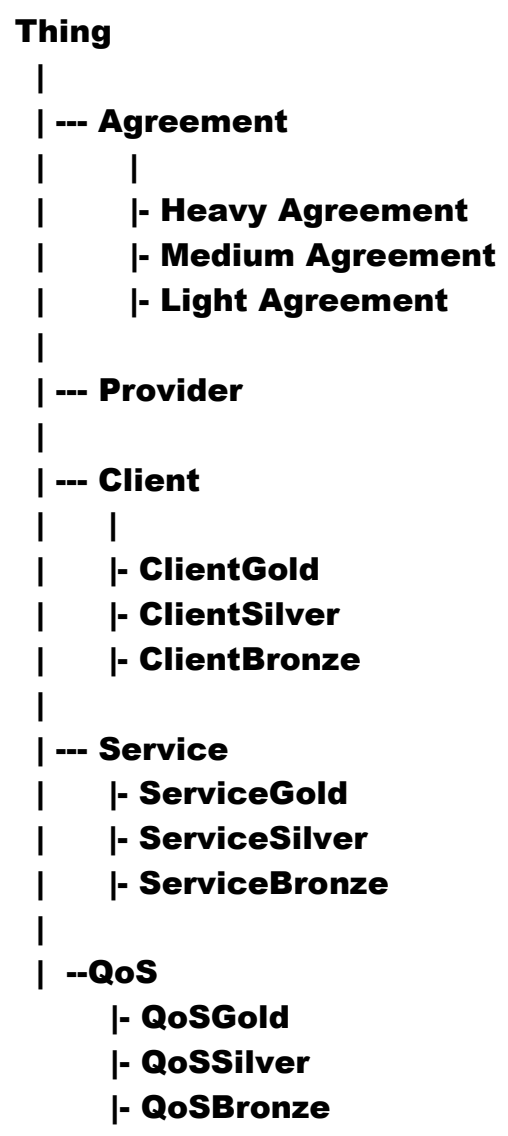




\section{$\underline{\text { Diagramas de Relacões Binárias (Binary-Relations Diagrams) }}$}

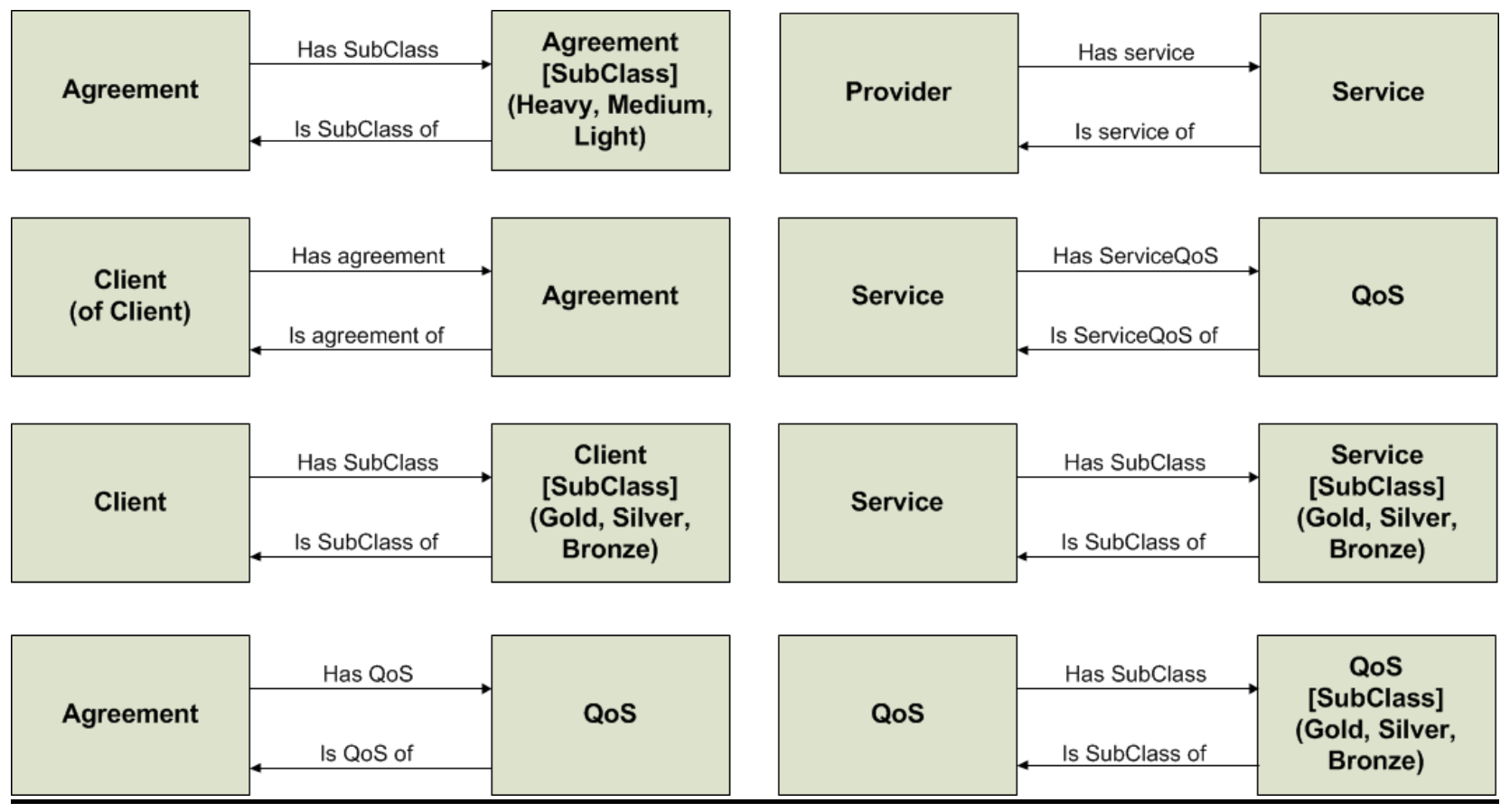




\section{Dicionário de Conceitos (Concept Dictionary)}

\begin{tabular}{|c|c|c|c|c|c|}
\hline $\begin{array}{l}\text { Nome do } \\
\text { Conceito }\end{array}$ & Sinônimo & $\begin{array}{l}\text { Acrônimo/ } \\
\text { Sigla }\end{array}$ & Instâncias & Atributos & Relações \\
\hline Agreement & - & Agmt & [Name]Agreement & $\begin{array}{l}\text { QoS } \\
\text { AgreementFine } \\
\text { AgreementName }\end{array}$ & $\begin{array}{l}\text { hasQoS, } \\
\text { isAgreementOf }\end{array}$ \\
\hline Client & Agent & - & [Name]Client & $\begin{array}{l}\text { ClientName, } \\
\text { ClientDescription, } \\
\text { ClientAddress, } \\
\text { Agreement }\end{array}$ & hasAgreement \\
\hline Provider & - & - & [Name]Provider & $\begin{array}{l}\text { ProviderName, } \\
\text { ProviderAddress, } \\
\text { ProviderCompany, } \\
\text { Services }\end{array}$ & hasService \\
\hline Service & - & - & [Name]Service & $\begin{array}{l}\text { ServiceName, } \\
\text { ServiceDescription, } \\
\text { ServiceAddress, } \\
\text { ServiceCompany, } \\
\text { ServiceDeveloper, } \\
\text { Servicelnterface, } \\
\text { QoS } \\
\text { Provider }\end{array}$ & hasServiceQoS \\
\hline QoS & - & - & [Name]QoS & $\begin{array}{l}\text { Accessibility } \\
\text { Accuracy } \\
\text { Availability } \\
\text { Capacity } \\
\text { Cost } \\
\text { Integrity } \\
\text { Interoperability } \\
\text { Latency } \\
\text { Performance } \\
\text { Reliability } \\
\text { Response Time } \\
\text { Robustness } \\
\text { Scalability } \\
\text { Security } \\
\text { Stability } \\
\text { Throughput }\end{array}$ & $\begin{array}{l}\text { IsServiceQoSof } \\
\text { IsQoSOf }\end{array}$ \\
\hline
\end{tabular}




\section{$\underline{\text { Tabelas de Relações Binárias (Binary-Relations Tables) }}$}

\begin{tabular}{|l|l|}
\hline Nome da Relação & hasAgreement \\
\hline Conceito fonte & Client \\
\hline Cardinalidade Fonte & $(\mathbf{1 , 1 )}$ \\
\hline Conceito Alvo & Agreement \\
\hline Cardinalidade Alvo & $(\mathbf{1 , 1 )}$ \\
\hline Propriedades Matemáticas & - \\
\hline Relação inversa & isAgreementof \\
\hline
\end{tabular}

\begin{tabular}{|l|l|}
\hline Nome da Relação & hasQoS \\
\hline Conceito fonte & Agreement \\
\hline Cardinalidade Fonte & $(1,1)$ \\
\hline Conceito Alvo & QoS \\
\hline Cardinalidade Alvo & $(1,1)$ \\
\hline Propriedades Matemáticas & - \\
\hline Relação inversa & isQoSOf \\
\hline
\end{tabular}

\begin{tabular}{|l|l|}
\hline Nome da Relação & hasService \\
\hline Conceito fonte & Provider \\
\hline Cardinalidade Fonte & $(\mathbf{1}, \mathbf{n})$ \\
\hline Conceito Alvo & Service \\
\hline Cardinalidade Alvo & $(\mathbf{n}, \mathbf{1})$ \\
\hline Propriedades Matemáticas & - \\
\hline Relação inversa & isServiceOf \\
\hline
\end{tabular}

\begin{tabular}{|l|l|}
\hline Nome da Relação & hasServiceQoS \\
\hline Conceito fonte & Service \\
\hline Cardinalidade Fonte & $(\mathbf{1 , 1})$ \\
\hline Conceito Alvo & QoS \\
\hline Cardinalidade Alvo & $(\mathbf{1 , 1 )}$ \\
\hline Propriedades Matemáticas & - \\
\hline Relação inversa & isServiceQoSof \\
\hline
\end{tabular}


$\underline{\text { Tabela de Instâncias (Instance Table) }}{ }^{1}$

\begin{tabular}{|l|l|l|}
\hline \multicolumn{1}{|c|}{ Instância } & \multicolumn{1}{c|}{ Atributo } & \multicolumn{1}{c|}{ Valor } \\
\hline \multirow{2}{*}{ [ClientName]Agreement } & hasName & [Name]Agreement \\
\cline { 2 - 3 } (Acordos) & hasFine & (Double) - Valor da Multa \\
\cline { 2 - 3 } & hasQos & [ClientName]QoS \\
\hline \multicolumn{2}{|c|}{ Instância } & \multicolumn{1}{c|}{ Atributo } \\
\hline \multirow{2}{*}{ Vame]Client[Number] } & hasClientDescription & (String) \\
\cline { 2 - 3 } & hasClientAddress & (String) - Endereço IP \\
\cline { 2 - 3 } (Clientes) & hasClientName & (String) - Mesmo da Instância \\
\cline { 2 - 3 } & hasAgreement & [ClientName]Agreement \\
\hline
\end{tabular}

\begin{tabular}{|l|l|l|}
\hline \multicolumn{1}{|c|}{ Instância } & \multicolumn{1}{|c|}{ Atributo } & \multicolumn{1}{c|}{ Valor } \\
\hline \multirow{3}{*}{ Name]Provider[Number] } & hasProviderName & (String) -Mesmo da Instância \\
\cline { 2 - 3 } & hasProviderAddress & (String) - Endereço IP \\
\cline { 2 - 3 } (Provedores) & hasProviderCompany & (String) - USP[Number] \\
\cline { 2 - 3 } & hasService & {$[$ Name]Service[Number] } \\
\hline
\end{tabular}

\begin{tabular}{|l|l|l|}
\hline \multicolumn{1}{|c|}{ Instância } & \multicolumn{1}{|c|}{ Atributo } & \multicolumn{1}{c|}{ Valor } \\
\hline \multirow{4}{*}{ Name]Service[Number] } & hasServiceName & (String) -Mesmo da Instância \\
\cline { 2 - 3 } & hasServiceAddress & (String) - Endereço IP \\
\cline { 2 - 3 } & hasServicelnterface & (String) - WSDL \\
\cline { 2 - 3 } & hasServiceDescription & (String) \\
\cline { 2 - 3 } & hasServiceDeveloper & (String) - Luis Nakamura \\
\cline { 2 - 3 } (Serviços) & hasServiceCompany & (String) - USP[Number] \\
\cline { 2 - 3 } & hasServiceQoS & [Name]Service[Number]QoS \\
\hline
\end{tabular}

\begin{tabular}{|l|l|l|}
\hline \multicolumn{1}{|c|}{ Instância } & \multicolumn{1}{|c|}{ Atributo } & \multicolumn{1}{c|}{ Valor } \\
\hline [Name]Service[Number]QoS & $\begin{array}{l}\text { Todos os atributos da tabela } \\
\text { de QoS (Lee e Shin, 2008): } \\
\text { (QoS dos Serviços) }\end{array}$ & $\begin{array}{l}\text { (Double) - Nível do atributo } \\
\text { de QoS. }\end{array}$ \\
\hline
\end{tabular}

\begin{tabular}{|l|l|l|}
\hline \multicolumn{1}{|c|}{ Instância } & \multicolumn{1}{c|}{ Atributo } & \multicolumn{1}{c|}{ Valor } \\
\hline [NameClient]QoS & $\begin{array}{l}\text { Todos os atributos da tabela } \\
\text { de QoS (Lee e Shin, 2008): } \\
\text { (QoS dos Clientes) }\end{array}$ & $\begin{array}{l}\text { (Double) - Nível do atributo } \\
\text { de QoS. }\end{array}$ \\
\hline
\end{tabular}

\footnotetext{
${ }^{1}$ Não são listadas todas as instâncias, pois na ontologia há mais de mil instâncias (por exemplo, ontologia com 1200 serviços e 30 clientes). Apenas o padrão e valores adotados são exibidos.
} 
Tabelas de Atributos das Instâncias (Instance-Attribute Table)

\begin{tabular}{|l|l|}
\hline Nome do atributo & hasAgreementFine \\
\hline Tipo de Valor (Tipo de dado) & Double \\
\hline Intervalo de valores & - \\
\hline Valor Padrão & - \\
\hline Cardinalidade & $(1,1)$ \\
\hline
\end{tabular}

\begin{tabular}{|l|l|}
\hline Nome do atributo & hasAgreementName \\
\hline Tipo de Valor (Tipo de dado) & String \\
\hline Intervalo de valores & - \\
\hline Valor Padrão & - \\
\hline Cardinalidade & $(1,1)$ \\
\hline
\end{tabular}

\begin{tabular}{|l|l|}
\hline Nome do atributo & hasClientName \\
\hline Tipo de Valor (Tipo de dado) & String \\
\hline Intervalo de valores & - \\
\hline Valor Padrão & - \\
\hline Cardinalidade & $(1,1)$ \\
\hline
\end{tabular}

\begin{tabular}{|l|l|}
\hline Nome do atributo & hasClientAddess \\
\hline Tipo de Valor (Tipo de dado) & String \\
\hline Intervalo de valores & - \\
\hline Valor Padrão & - \\
\hline Cardinalidade & $(1,1)$ \\
\hline
\end{tabular}

\begin{tabular}{|l|l|}
\hline Nome do atributo & hasClientDescription \\
\hline Tipo de Valor (Tipo de dado) & String \\
\hline Intervalo de valores & - \\
\hline Valor Padrão & - \\
\hline Cardinalidade & $(1,1)$ \\
\hline
\end{tabular}

\begin{tabular}{|l|l|}
\hline Nome do atributo & hasServiceCompany \\
\hline Tipo de Valor (Tipo de dado) & String \\
\hline Intervalo de valores & - \\
\hline Valor Padrão & - \\
\hline Cardinalidade & $(1,1)$ \\
\hline
\end{tabular}

\begin{tabular}{|l|l|}
\hline Nome do atributo & hasServiceDeveloper \\
\hline Tipo de Valor (Tipo de dado) & String \\
\hline Intervalo de valores & - \\
\hline Valor Padrão & - \\
\hline Cardinalidade & $(1,1)$ \\
\hline
\end{tabular}

\begin{tabular}{|l|l|}
\hline Nome do atributo & hasServicelnterface \\
\hline Tipo de Valor (Tipo de dado) & String \\
\hline Intervalo de valores & - \\
\hline Valor Padrão & - \\
\hline Cardinalidade & $(1,1)$ \\
\hline
\end{tabular}

\begin{tabular}{|l|l|}
\hline Nome do atributo & hasServiceAddress \\
\hline Tipo de Valor (Tipo de dado) & String \\
\hline Intervalo de valores & - \\
\hline Valor Padrão & - \\
\hline Cardinalidade & $(1,1)$ \\
\hline \begin{tabular}{|l|l|}
\hline Nome do atributo & hasServiceDescription \\
\hline Tipo de Valor (Tipo de dado) & String \\
\hline Intervalo de valores & - \\
\hline Valor Padrão & - \\
\hline Cardinalidade & $(1,1)$ \\
\hline
\end{tabular}
\end{tabular}




\begin{tabular}{|c|c|}
\hline Nome do atributo & hasServiceName \\
\hline Tipo de Valor (Tipo de dado) & String \\
\hline Intervalo de valores & - \\
\hline Valor Padrão & - \\
\hline Cardinalidade & $(1,1)$ \\
\hline Nome do atributo & hasProviderAddress \\
\hline Tipo de Valor (Tipo de dado) & String \\
\hline Intervalo de valores & - \\
\hline Valor Padrão & - \\
\hline Cardinalidade & $(1,1)$ \\
\hline Nome do atributo & hasProviderCompany \\
\hline Tipo de Valor (Tipo de dado) & String \\
\hline Intervalo de valores & - \\
\hline Valor Padrão & - \\
\hline Cardinalidade & $(1,1)$ \\
\hline Nome do atributo & hasProviderName \\
\hline Tipo de Valor (Tipo de dado) & String \\
\hline Intervalo de valores & - \\
\hline Valor Padrão & - \\
\hline Cardinalidade & $(1,1)$ \\
\hline
\end{tabular}

\begin{tabular}{|c|c|}
\hline Nome do atributo & has[Atributo_de_QoS]ContentValue \\
\hline Tipo de Valor (Tipo de dado) & Double \\
\hline Intervalo de valores & - \\
\hline Valor Padrão & - \\
\hline Cardinalidade & $(1,1)$ \\
\hline Nome do atributo & hasAgreement \\
\hline Tipo de Valor (Tipo de dado) & Agreement \\
\hline Intervalo de valores & - \\
\hline Valor Padrão & - \\
\hline Cardinalidade & $(1,1)$ \\
\hline Nome do atributo & hasQoS \\
\hline Tipo de Valor (Tipo de dado) & QoS \\
\hline Intervalo de valores & - \\
\hline Valor Padrão & - \\
\hline Cardinalidade & $(1,1)$ \\
\hline Nome do atributo & hasService \\
\hline Tipo de Valor (Tipo de dado) & Service \\
\hline Intervalo de valores & - \\
\hline Valor Padrão & - \\
\hline Cardinalidade & $(1, n)$ \\
\hline Nome do atributo & hasServiceQoS \\
\hline Tipo de Valor (Tipo de dado) & QoS \\
\hline Intervalo de valores & - \\
\hline Valor Padrão & - \\
\hline Cardinalidade & $(1,1)$ \\
\hline
\end{tabular}




\section{ANEXO 3}

\section{Anexo 3.1 - Criação de alguns elementos da ontologia do módulo UDOnt-Q}

Os primeiros elementos criados na ontologia do módulo UDOnt-Q foram os provedores. Cinco provedores foram cadastrados, este número foi escolhido pelo fato dos experimentos contarem apenas com cinco servidores disponíveis. Para atender a todas as possibilidades dos experimentos foram criados vários arquivos da mesma ontologia. Para o fator número de clientes há duas opções: 15 clientes e 30 clientes. Portanto, hora foram cadastrados 15 clientes hora 30 clientes na ontologia. Para facilitar a execução dos experimentos foram criados dois arquivos da ontologia para atender essas duas possibilidades.

$\mathrm{Na}$ ontologia com 15 clientes foram criados:

\section{- 1 Cliente Gold.}

- 7 Clientes Silver.

- 7 Clientes Bronze.

Na ontologia com 30 clientes foram criados:

\section{- 1 Cliente Gold.}

- 15 Clientes Silver.

- 14 Clientes Bronze.

Para a criação de cada cliente na ontologia foi criado um acordo para ser associado a ele. Seguindo essa sequencia, para cada acordo criado foi criada uma QoS.

O número de Web Services cadastrados na ontologia pode assumir três valores (300,600 e 1200) dependendo do experimento executado. Novamente, para facilitar os experimentos foram criadas três variações do arquivo da ontologia. Para cada uma dessas variações havia duas opções para o número de clientes (15 e 30). Dessa forma, foram criados seis arquivos da ontologia.

Para cada Web Service cadastrado foi criada e associada uma QoS que quando inferida seria classificada em uma subclasse do tipo Gold, Silver ou Bronze. Os valores dos atributos de cada QoS foram gerados aleatoriamente, porém esses valores ficam dentro de um limite pré-definido pelo programador. Dessa forma, o programado poderia determinar qual seria a subclasse de QoS daquela determinada QoS que estava sendo criado e consequentemente a subclasse do Web Service.

Apenas uma pequena quantidade de Web Services poderia se encaixar na pesquisa dos algoritmos do UDOnt-Q. Portanto, o programador definiu que apenas $10 \%$ dos serviços teriam o "nome de busca" utilizados pelos clientes, os outros serviços teriam "outro nome" diferente do nome de serviço que os clientes desejavam. Como nos experimentos houve requisições de vários tipos de 
clientes (Gold, Silver, Bronze) os serviços foram igualmente divididos nestas três categorias. A figura a seguir exibe um exemplo da forma de como foram criados as QoS e Web Services para a ontologia com 300 serviços. Analisando a figura a seguir, nota-se que o programador cadastrava os serviços em cinco ciclos de 60, totalizando 300 serviços. Em cada ciclo de 60 serviços foram cadastrados 6 (seis) serviços com o nome procurado e outros 54 com outro nome. No total são 300 serviços, porém apenas 30 serviços possuem o nome da busca (10\%) e estão divididos nas três subclasses (10 por subclasse). Da mesma forma as ontologias com 600 e 1200 serviços foram criadas.

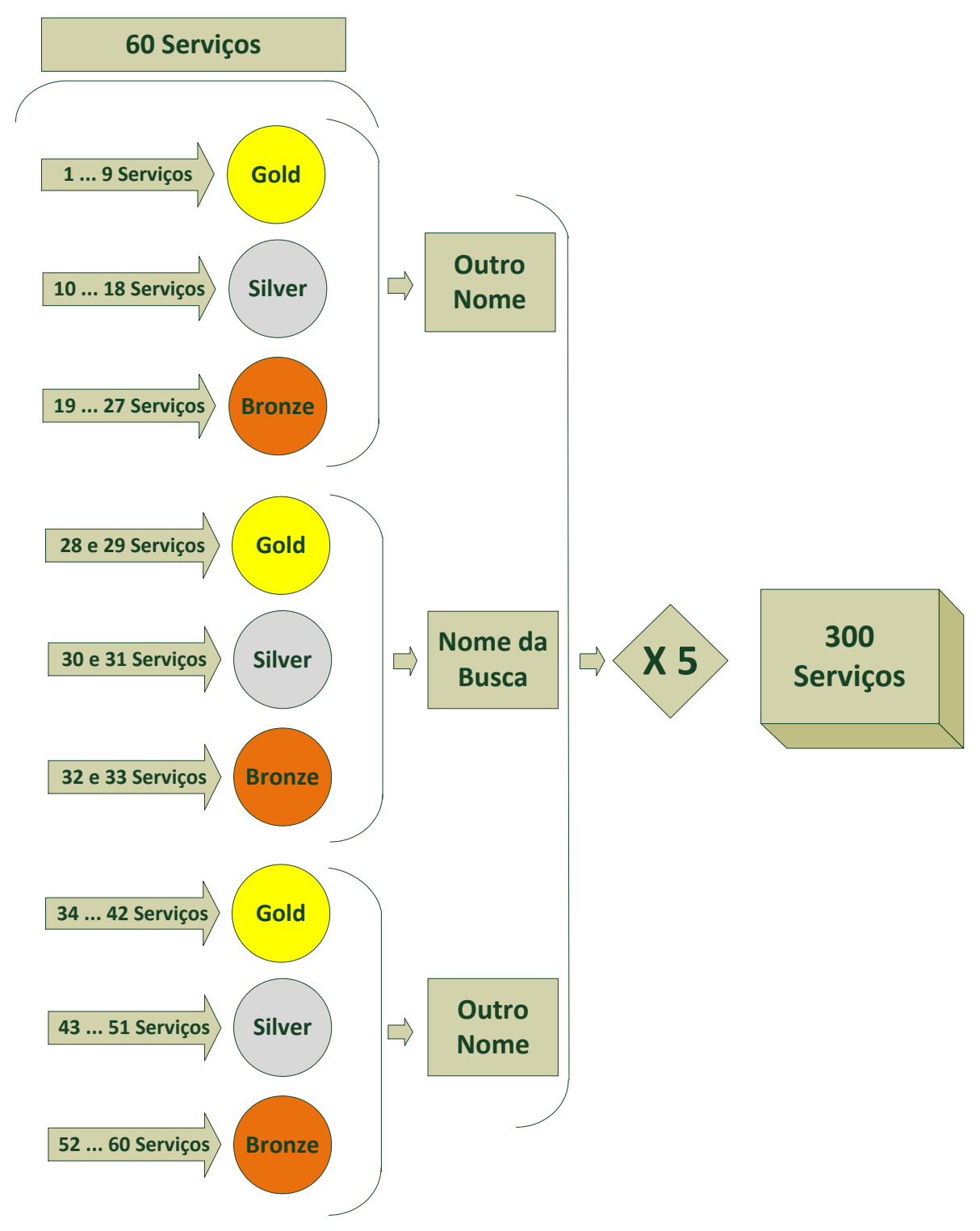

Esquema da criação de 300 serviços na ontologia. 


\section{ANEXO 4}

Anexo 4.1 - Resultados dos Conjuntos de Experimentos (CJE) 3 e 4

Fatorial completo $2^{3}$ e Médias do Tempo de Busca (TB)do CJE-3

\begin{tabular}{|c|c|c|c|c|c|c|}
\hline Experimento & $\begin{array}{c}\text { Fator A } \\
\text { (N. Serviços) }\end{array}$ & $\begin{array}{c}\text { Fator B } \\
\text { (N. Clientes) }\end{array}$ & $\begin{array}{c}\text { Fator C } \\
\text { (Algoritmo) }\end{array}$ & TB Média & RTC Média & Throughput \\
\hline 1 & 300 & 15 & Object & 0,10664 & 0,36866 & 2,71 \\
\hline 2 & 300 & 15 & SPARQL & 0,42057 & 0,72796 & 1,37 \\
\hline 3 & 300 & 30 & Object & 0,29714 & 0,59309 & 1,69 \\
\hline 4 & 300 & 30 & SPARQL & 1,09020 & 1,42669 & 0,70 \\
\hline 5 & 600 & 15 & Object & 0,15821 & 0,46365 & 2,16 \\
\hline 6 & 600 & 15 & SPARQL & 1,32245 & 1,61105 & 0,62 \\
\hline 7 & 600 & 30 & Object & 0,35368 & 0,68893 & 1,45 \\
\hline 8 & 600 & 30 & SPARQL & 2,80296 & 3,22292 & 0,31 \\
\hline
\end{tabular}

Fatorial completo $2^{3}$ e Médias do Tempo de Busca (TB)do CJE-4

\begin{tabular}{|c|c|c|c|c|c|c|}
\hline Experimento & $\begin{array}{c}\text { Fator A } \\
\text { (N. Serviços) }\end{array}$ & $\begin{array}{c}\text { Fator B } \\
\text { (N. Clientes) }\end{array}$ & $\begin{array}{c}\text { Fator C } \\
\text { (Algoritmo) }\end{array}$ & TB Média & RTC Média & Throughput \\
\hline 1 & 300 & 15 & Object & 0,10664 & 0,3687 & 2,71 \\
\hline 2 & 300 & 15 & SPARQL & 0,42057 & 0,7280 & 1,37 \\
\hline 3 & 300 & 30 & Object & 0,29714 & 0,5931 & 1,69 \\
\hline 4 & 300 & 30 & SPARQL & 1,09020 & 1,4267 & 0,70 \\
\hline 5 & 1200 & 15 & Object & 0,24107 & 0,5830 & 1,72 \\
\hline 6 & 1200 & 15 & SPARQL & 6,02424 & 6,3147 & 0,16 \\
\hline 7 & 1200 & 30 & Object & 0,46384 & 0,8131 & 1,23 \\
\hline 8 & 1200 & 30 & SPARQL & 12,44431 & 12,8750 & 0,08 \\
\hline
\end{tabular}

